Espaços de configurações

Cesar Augusto Ipanaque Zapata 

Assinatura:

Cesar Augusto Ipanaque Zapata

\section{Espaços de configurações}

Dissertação apresentada ao Instituto de Ciências Matemáticas e de Computação - ICMC-USP, como parte dos requisitos para obtenção do título de Mestre em Ciências - Matemática. VERSÃO REVISADA

Área de Concentração: Matemática

Orientadora: Profa. Dra. Denise de Mattos

\section{USP - São Carlos}

Maio de 2017 
Ficha catalográfica elaborada pela Biblioteca Prof. Achille Bassi e Seção Técnica de Informática, ICMC/USP, com os dados fornecidos pelo(a) autor(a)

Zapata, Cesar Augusto Ipanaque
Espaços de configurações / Cesar Augusto
Ipanaque Zapata; orientadora Denise de Mattos . -
São Carlos - SP, 2017.
156 p.
Dissertação (Mestrado - Programa de Pós-Graduação
em Matemática) - Instituto de Ciências Matemáticas e
de Computação, Universidade de São Paulo, 2017.
1. Grupos de Homotopia. 2. Homologia singular.
3. Espaços de configurações. 4. Fibração de Fadell
e Neuwirth. 5. Sequências espectrais. I. , Denise de
Mattos, orient. II. Título.


Cesar Augusto Ipanaque Zapata

\section{Configuration spaces}

Master dissertation submitted to the Instituto de Ciências Matemáticas e de Computação - ICMCUSP, in partial fulfillment of the requirements for the degree of the Master Program in Mathematics. FINAL VERSION

Concentration Area: Mathematics

Advisor: Profa. Dra. Denise de Mattos

USP - São Carlos

May 2017 

A Yasmy e Zete. 

Os agradecimentos principais são direcionados, em especial, à minha orientadora professora Denise de Mattos. Também agradeço aos professores Edivaldo Lopes dos Santos, Eduardo Tengan e Oziride Manzoli Neto.

Agradeço ao Instituto de Ciências Matemáticas e de Computação da Universidade de São Paulo ${ }^{1}$ à Capes ${ }^{2}$ pelo apoio financeiro e a todos aqueles que contribuíram para que este trabalho fosse possível. 

"La información no es conocimiento; su única fuente es la experiencia." (Albert Einstein) 



\section{RESUMO}

ZAPATA, C. A. I.. Espaços de configurações . 2017. 156 f. Dissertação (Mestrado em Ciências - Matemática) - Instituto de Ciências Matemáticas e de Computação (ICMC/USP), São Carlos - SP.

O objetivo principal deste trabalho será apresentar um estudo detalhado dos espaços de configurações. Dissertaremos sobre: espaços de configurações clássicos, invariância do bordo, espaço de configurações para superfícies, fibração de Fadell e Neuwirth e espaços de configurações do espaço Euclideano, da esfera e do espaço projetivo complexo.

Palavras-chave: Grupos de Homotopia, Homologia singular, Espaços de configurações, Fibração de Fadell e Neuwirth, Sequências espectrais. 



\section{ABSTRACT}

ZAPATA, C. A. I.. Espaços de configurações . 2017. 156 f. Dissertação (Mestrado em Ciências - Matemática) - Instituto de Ciências Matemáticas e de Computação (ICMC/USP), São Carlos - SP.

The main objective of this work will be to present a detailed study of the configuration spaces. We will study: classical configuration spaces, invariance of the boundary, configuration spaces of surfaces, Fadell and Neuwirth fibration and configuration spaces of the Euclidean space and spheres.

Key-words: Homotopy groups, Singular Homology, Configuration spaces, Fadell-Neuwirth Fibration, Spectral sequences. 



\section{LISTA DE ILUSTRAÇÕES}

Figura 1 - Transformação natural . . . . . . . . . . . . . . . . . . 30

Figura 2 - Espaços que não são variedades . . . . . . . . . . . . . . . . 46

Figura 3 - Diferenciais . . . . . . . . . . . . . . . 50

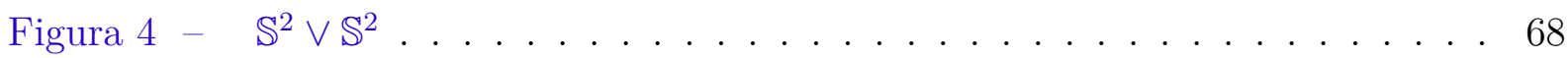

Figura 5 - Uma $n$-variedade com bordo . . . . . . . . . . . 70

Figura 6 - Vizinhança colarinho . . . . . . . . . . . . . 71

Figura 7 - Vizinhança colarinho . . . . . . . . . . . . . 73

Figura 8 - Isotopia entre $i \circ p$ e $I d_{M} \ldots \ldots \ldots \ldots$. . . . . . . . . . 73

Figura 9 - Disco fechado numa variedade . . . . . . . . . . . . 80

Figura 10 - Naturalidade da sequência exata longa de uma fibração. . . . . . . . . . 85

Figura 11 - Quase trivialização local da Fibração de Fadell e Neuwirth. . . . . . . . 87

Figura 12 - Trivialização local da Fibração de Fadell e Neuwirth. . . . . . . . . . . 88

Figura 13 - Isomorfismo $\Omega$. . . . . . . . . . . . . . . . . . . 98

Figura 14 - Sequência fundamental de fibrações. . . . . . . . . . . . . . . . . . 111

Figura 15 - Relação de seções . . . . . . . . . . . . . . . . . . . . . . . . 111

Figura $16-S F\left(\mathbb{S}^{n}, 2\right) \simeq \mathbb{R P}^{n} \ldots \ldots \ldots \ldots \ldots \ldots$. . . . . . . . . . . . . . . . . . . . .

Figura $17-S F\left(\mathbb{R}^{n+1}, 2\right) \simeq \mathbb{R P}^{n} \ldots \ldots \ldots \ldots \ldots \ldots \ldots$

Figura 18 - Diagrama comutativo . . . . . . . . . . . . . . 116

Figura 19 - Naturalidade da sequência exata longa da fibração. . . . . . . . . . . . 117

Figura 20 - Diagrama comutativo no caso não ordenado . . . . . . . . . . . . . 117

Figura 21 - Diagramas comutativos . . . . . . . . . . . . . . . . 118

Figura $22-E_{*, *}^{2} \ldots \ldots \ldots \ldots \ldots \ldots \ldots \ldots \ldots$

Figura 23 - Sequência espectral do espaço de configurações $F\left(\mathbb{R}^{n}, 4\right)$. . . . . . . 127

Figura 24 - Sequência espectral do espaço de configurações $F\left(\mathbb{C P}^{n}, 2\right)$. . . . . . . 128

Figura 25 - Lema dos quatro . . . . . . . . . . . . . . . . . . . . . 131

Figura 26 - Lema dos quatro . . . . . . . . . . . . . . . . . . 131

Figura 27 - Lema dos cinco . . . . . . . . . . . . . . . . . . . . . 132

Figura 28 - Naturalidade do homomorfismo de Hurewicz. . . . . . . . . . . . . . 136

Figura 29 - Naturalidade do recobrimento . . . . . . . . . . . . . . . . . . . . . . 144

Figura 30 - Naturalidade do diagrama da sequência associada a um recobrimento . 145

Figura 31 - Diagrama comutativo de aplicações quocientes. . . . . . . . . . . . . 147

Figura 32 - Usando a naturalidade da sequência exata longa de uma fibração. . . . 148

Figura 33 - Usando a naturalidade da sequência exata curta para um recobrimento. 148 

$X$ - Espaço topológico X

$\mathbb{R}^{n}$ — espaço euclideano

$\mathbb{R} \mathbb{P}^{n}$ - $n$-espaço projetivo real

$\mathbb{C P}^{n}$ - n-espaço projetivo complexo

$\mathbb{Z}$ — números inteiros

$\mathbb{N}$ - números naturais, inteiros positivos

$\pi_{n}$ - n-ésimo grupo de homotopia

$H_{q}(X ; R)-q$-ésimo grupo de Homologia

$\cong-$ Isomorfismo

$\equiv$ — bijeção entre conjuntos

$\simeq$ - equivalência homotópica

$\stackrel{w}{\simeq}$ - equivalência homotópica fraca

$I=[0,1]$ — intervalo unitário da reta 



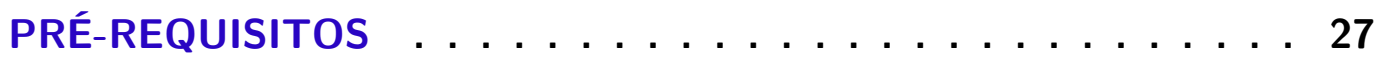

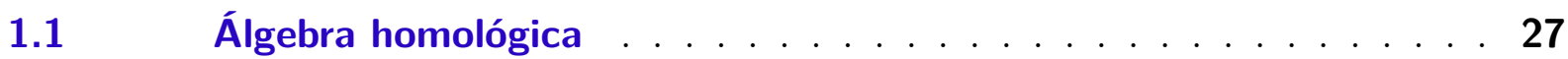

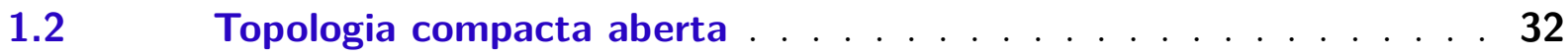

$1.3 \quad$ Grupos de transformações ou $G$-ações . . . . . . . . . . . . 38

$1.4 \quad$ Variedades . . . . . . . . . . . . . . . . . . . 44

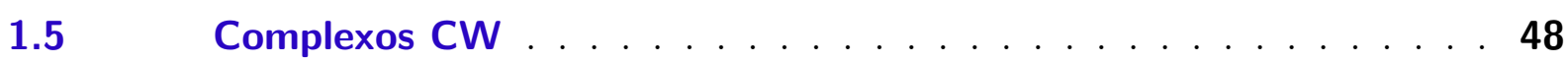

1.6 Sequências Espectrais . . . . . . . . . . . . . 49

1.6.1 Sequência espectral de Leray-Serre . . . . . . . . . . . . 51

$1.7 \quad$ Espaços classificantes . . . . . . . . . . . . . . . . 53

2 ESPAÇOS DE CONFIGURAÇÕES . . . . . . . . . 57

2.1 Espaços de Configurações clássicos . . . . . . . . . . . . 57

2.2 Espaços de configurações para variedades com bordo . . . . . . . 68

3 ESPAÇO DE CONFIGURAC̣ÕES PARA SUPERFÍCIES $\ldots \ldots \ldots 77$

3.1 Fibração de Fadell e Neuwirth . . . . . . . . . . . . 77

3.2 Grupos de Tranças e Espaços de Configurações . . . . . . . . . . . 93

4 SEÇÕES DA FIBRAÇÃO DE FADELL E NEUWIRTH . . . . . . . . 97

$4.1 \quad$ Os grupos de homotopia de $F\left(M \times \mathbb{R}^{n}, k\right) \ldots \ldots \ldots$

5 VARIEDADES DISTINTAS COM OS MESMOS ESPAÇOS DE CON-

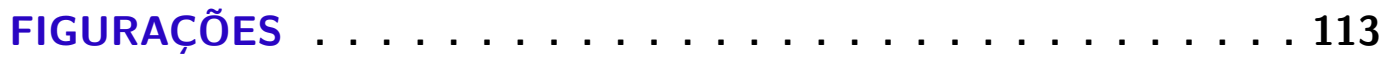

$5.1 \quad F(M, 2) \simeq F(N, 2)$ não implica $M \simeq N \ldots \ldots \ldots \ldots$

5.2 Espaços de configurações para variedades contráteis . . . . . . 115

6 ESPAÇOS DE CONFIGURAÇÕES PARA CERTAS VARIEDADES . 121

6.1 Espaços de configurações Euclideanos . . . . . . . . . . . 121

$6.2 \quad$ Espaços de configurações da esfera $\mathbb{S}^{n} \ldots \ldots \ldots 129 \ldots \ldots$

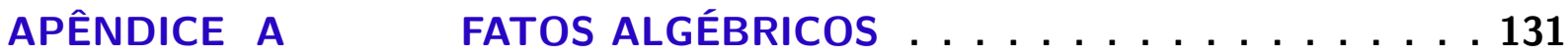

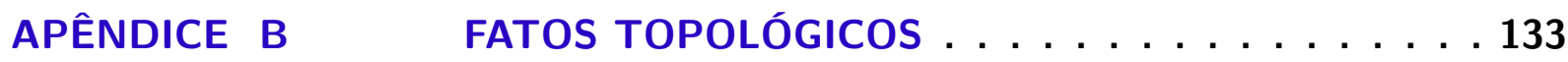

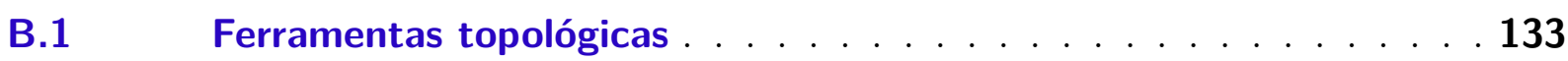


B.2 Variedades $m$-homogêneas . . . . . . . . . . . . . 136

B.3 Sequência exata curta para um recobrimento . . . . . . . . . . . 142

B.4 Variedades com buracos . . . . . . . . . . . . . 148

REFERÊNCIAS . . . . . . . . . . . . . . . . . 151 
Oscar Zariski, em 1937, utilizou pela primeira vez o conceito de espaços de configurações(ZARISKI, 1937). Posteriormente, Ralph Fox, em 1962, definiu o conceito de tranças geométricas usando os espaços de configurações (FOX; NEUWIRTH, 1962). Um estudo detalhado dos espaços de configurações, ordenados e não ordenados, foi apresentado no trabalho de Fadell e Neuwirth (FADELL; NEUWIRTH, 1962).

Uma das características interessantes dos espaços de configurações é a sua estreita relação com a teoria de tranças, introduzida por Emil Artin em 1926, a qual é uma grande linha de pesquisa ativa até o momento. Desde então, os espaços de configurações aparecem em distintas áreas de pesquisa: Geometria, Combinatória, Física, Química, Biologia e, mais recentemente, na Robótica.

O espaço de configurações ordenado de $k$ pontos distintos em um espaço topológico $X$ é o subespaço de $X^{k}$ definido por

$$
F(X, k):=\left\{\left(x_{1}, \ldots, x_{k}\right) \in X^{k}: x_{i} \neq x_{j} \quad \text { se } i \neq j\right\} .
$$

Uma das propriedades básicas dos espaços de configurações é a existência de uma ação livre do grupo simétrico sobre $F(X, k)$. O espaço de configurações não ordenado, denotado por $S F(X, k)$, é definido então como sendo o quociente de $F(X, k)$ pela ação natural do grupo simétrico $S_{k}$, a qual permuta coordenadas.

Um dos problemas em Topologia Algébrica consiste em se calcular a homologia e a cohomologia dos espaços de configurações.

(1988) Löffler e Milgram (MILGRAM; LÖFFLER, 1988) calcularam os $\mathbb{Z} / 2$-grupos de homologia de $S F(X, n)$, para qualquer variedade (fechada) $X$.

(1989) Bödigheimer (BÖDIGHEIMER; COHEN; TAYLOR, 1989) calculou os grupos de homologia de $S F(X, n)$, para qualquer variedade $X$ de dimensão ímpar.

Em ambos casos, tais grupos de homologia dependem apenas da homologia da variedade $X$ e de sua dimensão. Em contraste, os números de Betti racionais de $S F(X, n)$ não são determinados pelos números de Betti racionais de $X$, no caso em que $X$ é uma variedade de dimensão par. Quando se trata dos anéis de cohomologia de $S F(X, n)$, um dos problemas é conhecer se tal anel é um invariante homotópico para variedades fechadas $X$. 
(1991) Bendersky e Gitler (BENDERSKY; GITLER, 1991) observaram que os grupos de cohomologia de $F(X, n)$ e $S F(X, n)$ são invariantes homotópicos para variedades $X$ fechadas orientáveis e para variedades formais racionais $X$, tais como variedades projetivas complexas suaves. Eles calcularem os grupos de cohomologia racional de $F(X, n)$ e $S F(X, n)$, explicitamente, em termos do anel de cohomologia racional da variedade $X$.

(1996) Burt Totaro (TOTARO, 1996) mostrou que se $X$ é uma variedade projetiva complexa suave, então os anéis de cohomologia racionais de $F(X, n)$ e $S F(X, n)$ podem ser calculados a partir do anel de cohomologia racional de $X$.

A seguir, faremos uma descrição detalhada de todos os resultados apresentados nos Capítulos 2 a 6 deste trabalho.

Os espaços de configurações de variedades topológicas também são variedades topológicas. E no caso de uma variedade suave conexa, os seus espaços de configurações possuem estrutura de complexos $C W$ (Teorema 2.1.19 e Corolário 2.1.21).

Para uma variedade topológica com bordo compacta e conexa $M$, a inclusão do interior de $M$ em $M$ induz equivalências de homotopia entre os espaços de configurações $F($ Int $M, k), F(M, k)$ e $S F($ Int $M, k)$ e $S F(M, k)$. Isso significa, que a menos de homotopia, o bordo de uma variedade é irrelevante para os espaços de configurações, assim é suficiente calcular os espaços de configurações para variedades topológicas sem bordo (Teorema 2.2.13)

A fibração de Fadell e Neuwirth é a ferramenta central no estudo da topologia e geometria dos espaços de configurações. Para uma $m$-variedade topológica sem bordo conexa $M, m \geq 2$, a aplicação:

$$
\begin{aligned}
\pi_{k, r}: \quad \begin{array}{l}
F(M, k) \\
\left(x_{1}, \ldots, x_{r}, \ldots, x_{k}\right)
\end{array} & \mapsto F(M, r) \\
& \left.\mapsto x_{1}, \ldots, x_{r}, \ldots, x_{k}\right):=\left(x_{1}, \ldots, x_{r}\right)
\end{aligned}
$$

obtida pela projeção sobre os primeiros $r$-fatores é um fibrado localmente trivial, com fibra $F\left(M-Q_{r}, k-r\right)$, onde $k>r \geq 1$ (Teorema 3.1.25).

Tal resultado permite mostrar, por exemplo, que para uma variedade topológica conexa $M$ com a propriedade que $M$ permanece conexa quando é perfurada em $k-1 \geq 0$ pontos, então os espaços de configurações de $M$ são conexo por caminhos (Corolário 3.1.27). Como consequência, teremos que os espaços de configurações (ordenados) do $\mathbb{R}^{n}$ e da $n$-esfera $\mathbb{S}^{n}$ são simplesmente conexos, para todo $n \geq 3$. Além disso, o espaço de configurações $F\left(\mathbb{C P}^{n}, 2\right)$ do espaço projetivo complexo $\mathbb{C P}^{n}$ é 1-conexo (Exemplos 3.1.30, 3.1 .31 e 3.1 .32 ).

Uma outra aplicação significativa da fibração de Fadell e Neuwirth é que se $M$ é o plano Euclideano ou se $M$ for uma superfície fechada, orientável ou não, então os espaços 
de configurações de $M-Q_{m}$ são espaços de Eilenberg-MacLane do tipo $K(\pi, 1)$, onde $Q_{m}$ é um subconjunto de $M$ contendo $m$ pontos. Além disso, se $M$ for diferente da esfera $\mathbb{S}^{2}$ e do plano projetivo $\mathbb{R P}^{2}$, então os espaços de configurações de $M$ são $K(\pi, 1)$-espaços, para todo $k \geq 1$ (Teorema 3.1.38). Uma consequência importante deste resultado é que se $M$ é o plano Euclideano ou se $M$ for uma superfície fechada, exceto a 2-esfera $\mathbb{S}^{2}$ e o plano projetivo $\mathbb{R P}^{2}$, o tipo de homotopia de $F(M, k)$ e $S F(M, k)$ é completamente determinado por seu grupo fundamental. Por essa razão, um interessante problema em Topologia Algébrica consiste em se calcular tais grupos de homotopia, os quais são definidos como grupos de tranças de superfícies

O grupo de tranças puras sobre $n$-cordas sobre uma variedade topológica $M$, de dimensão maior ou igual a $2, P B_{n}(M)$ é definido como sendo o grupo fundamental do espaço de configurações $F(M, k)$, e o grupo de tranças sobre $n$-cordas de $M, B_{n}(M)$ é definido como sendo o grupo fundamental do espaço de configurações $S F(M, k)$. Para uma variedade suave conexa $M$, com $\operatorname{dim} M \geq 3$, temos:

$$
P B_{k}(M):=\pi_{1}(F(M, k)) \cong \prod_{i=1}^{k} \pi_{1}(M), \quad \forall k \geq 1,
$$

ou seja, o grupo de tranças puras $P B_{k}(M)$ é determinado pelo grupo fundamental $\pi_{1}(M)$. Em particular, se $M$ for simplesmente conexa, então $F(M, k)$ será simplesmente conexo, ou seja,

$$
P B_{k}(M):=\pi_{1}(F(M, k))=1, \quad \forall k \geq 1
$$

Consequentemente,

$$
B_{k}(M):=\pi_{1}(S F(M, k))=\pi_{1}\left(F(M, k) / S_{k}\right) \cong S_{k}, \quad \forall k \geq 1 .
$$

Por essa razão, o estudo dos grupos de tranças possui relevância no caso de variedades de dimensão igual a 2 (Corolário 3.1.44 e Teorema B.3.2).

No que concerne aos grupos de homologia e homotopia dos espaços de configurações, para uma variedade topológica conexa $M$, com $\operatorname{dim}(M) \geq 1$, sempre que $\pi_{1}(M)$ for um grupo abeliano não trivial, então o grupo de homologia $H_{1}\left(F\left(M \times \mathbb{R}^{n}, k+1\right), \mathbb{Z}\right)$ e os grupos de homotopia $\pi_{1}\left(F\left(M \times \mathbb{R}^{n}, k+1\right)\right)$ são não triviais, para todo $n \geq 2 \mathrm{e}$ $k \geq 1$ (Corolário 4.1.22). Por exemplo, se $M$ é o grupo ortogonal especial $S O(n)$, então $H_{1}\left(F\left(S O(n) \times \mathbb{R}^{n}, k+1\right), \mathbb{Z}\right)$ é isomorfo à soma direta $\bigoplus_{1}^{k+1} \mathbb{Z}_{2}$, para todo $n \geq 3$ (Exemplo 4.1.25).

Uma outra relevante propriedade da fibração de Fadell e Neuwirth é sobre a existência de seções. Usando este fato é possível calcular os grupos de homotopia dos espaços de configurações de uma variedade topológica conexa, em termos dos grupos de homotopia da variedade perfurada (Teorema 4.1.27 e Corolário 4.1.32).

Uma outra questão natural que surge sobre os espaços de configurações é que para $M$ e $N$ espaços topológicos arbitrários não vazios, se $F(M, k)$ e $F(N, k)$ são do mesmo 
tipo, ou seja, são homeomorfos, ou possuem o mesmo tipo de homotopia, ou possuem o mesmo tipo de homotopia fraca, então $M$ e $N$ serão do mesmo tipo? Uma resposta trivial a essa questão, é obtida quando consideramos os espaços $\{$ pto $\}$ e $\{p t o, p t o\}$. Para tais espaços, claramente não homeomorfos, tem-se $F(\{p t o\}, k)=\emptyset=F(\{p t o, p t o\}, k), \forall k \geq 3$. Agora, no caso de uma $n$-variedade contrátil, sem bordo, com $n \geq 3$, seus espaços de configurações, ordenados e não ordenados, possuem o mesmo tipo de homotopia fraca que os correspondentes espaços de configurações do espaço Euclideano $\mathbb{R}^{n}$. Em particular, toda $n$-variedade contrátil, sem bordo possui o mesmo tipo de homotopia fraca que o espaço Euclideano $\mathbb{R}^{n}$. Um exemplo importante é dado pela variedade de Whitehead, a qual é uma 3-variedade aberta e contrátil, mas não é homeomorfa ao espaço Euclideano $\mathbb{R}^{3}$. Os espaços de configurações, ordenados e não ordenados, da variedade de Whitehead possuem o mesmo tipo de homotopia fraca que os correspondentes espaços de configurações do espaço Euclideano $\mathbb{R}^{3}$. Isto implica, em particular, que a variedade de Whitehead tem o mesmo tipo de homotopia fraca que o $\mathbb{R}^{3}$, porém a variedade de Whitehead não é homeomorfa a $\mathbb{R}^{3}$ (Corolário 5.2.3 e Teorema 5.2.6)

Em relação aos espaços de configurações do espaço Euclideano $\mathbb{R}^{n+1}$, seguirá que tais espaços não são espaços de Eilenberg-MacLane do tipo $K(\pi, 1)$, para todo $n \geq 2 \mathrm{e}$ para todo $k \geq 2$ (Corolário 6.1.7).

No que se refere à homologia, para $q$ suficientemente grande, os $R$-módulos de homologia singular $H_{q}(F(M, k), R)$ dos espaços de configurações de variedades topológicas conexas são triviais (Proposição 6.1.9). Ainda como uma consequência da fibração de Fadell e Neuwirth e usando a sequência espectral de Leray-Serre associada a esta fibração, calculamos os grupos de homologia dos espaços de configurações $F\left(\mathbb{R}^{n}, 3\right), F\left(\mathbb{R}^{n}, 4\right)$ e $F\left(\mathbb{C P}^{n}, 2\right)$ (Exemplos 6.1.10, 6.1.12 e 6.1.14).

Uma das razões fundamentais em se conhecer os grupos de homologia de um espaço topológico é que um complexo $C W$ simplesmente conexo, cujos grupos de homologia são finitamente gerados, tem o mesmo tipo de homotopia de um complexo $C W$ tendo um número mínimo de células consistentes com seus grupos de homologia, isto é, uma $n$-célula para cada somando $\mathbb{Z}$ de $H_{n}$, e um par de células de dimensões $n$ e $n+1$, para cada somando $\mathbb{Z}_{k}$ de $H_{n}$. Com base neste fato e pelo cálculo dos grupos de homologia citados anteriormente, concluímos que $F\left(\mathbb{R}^{n}, 4\right)(n \geq 3)$ tem o mesmo tipo de homotopia de um $3(n-1)$-dimensional complexo $C W$ finito e que o espaço de configurações $F\left(\mathbb{C P}^{n}, 2\right)$ tem o mesmo tipo de homotopia de um $2(2 n-1)$-dimensional CW complexo finito. (Exemplos $6.1 .18,6.1 .19)$.

O Capítulo 1 apresenta resultados essenciais para o desenvolvimento deste trabalho a saber: Categorias e Funtores, a Topologia Compacta Aberta, os Grupos de transformações, Complexos $C W$, Sequências Espectrais e Espaços Classificantes. Um resultado importante mostrado na Seção 1.6 é que todo espaço $C W$ do tipo $K(G, 1)$, com $G$ um grupo discreto 
enumerável, é um espaço classificante para $G$ (Proposição 1.7.10).

No Apêndice $A$ apresentamos dois resultados básicos de Álgebra Homológica: o Lema dos Quatro e o Lema dos Cinco.

No apêndice $B$, destacamos teoremas imprescindíveis que serão usados neste trabalho, tais como o Teorema da Invariância do Domínio, a Sequência de Mayer-Vietoris e o Teorema de Hurewicz. Além disso, apresentamos uma demonstração de que toda variedade topológica conexa $M$, com $\operatorname{dim} M \geq 2$, é $m$-homogênea (Definição B.2.1), para todo $m$ (Teorema B.2.9). Este resultado notável foi afirmado por Fadell e Neuwirth em (FADELL; NEUWIRTH, 1962). 

CAPÍTULO

\section{1}

PRÉ-REQUISITOS

Este capítulo introduz as noções básicas e notações que serão usadas em todo este trabalho. Em todo este capítulo, $R$ denotará um anel comutativo com unidade 1.

\section{$1.1 \quad$ Álgebra homológica}

Definição 1.1.1. ((SELICK, 2008), Definition 1.1.1, pg. 3 e (MACLANE, 1971), Chapter I, Section 2, pg. 10) Uma categoria $\mathcal{C}$ consiste de:

$\left(C_{1}\right)$ Uma classe $\operatorname{Obj} \mathcal{C}$, conhecida como os objetos de $\mathcal{C}$ (os quais não precisam ser um conjunto).

$\left(C_{2}\right)$ Para cada par $X, Y$ de objetos de $\operatorname{Obj} \mathcal{C}$, um conjunto, denotado $\mathcal{C}(X, Y)$ ou $\operatorname{Hom}_{\mathcal{C}}(X, Y)$, chamados os morfismos na categoria $\mathcal{C}$ de $X$ para $Y$.

$\left(C_{3}\right)$ Para cada terna $X, Y, Z$ de objetos de $\operatorname{Obj} \mathcal{C}$, uma aplicação

$$
\circ: \mathcal{C}(X, Y) \times \mathcal{C}(Y, Z) \longrightarrow \mathcal{C}(X, Z)
$$

chamada composição, onde $\circ(f, g)$ usualmente é denota por $g \circ f$ ou $g f$.

$\left(C_{4}\right)$ Para cada objeto $X$ de $\operatorname{Obj} \mathcal{C}$, um elemento $1_{X} \in \mathcal{C}(X, X)$, chamado o morfismo identidade de $X$, denotado também por $I d_{X}$.

Tais objetos e morfismos satisfazem as seguintes condições:

$\left(A_{1}\right) h \circ(g \circ f)=(h \circ g) \circ f$, para todo morfismo $f \in \mathcal{C}(W, X), g \in \mathcal{C}(X, Y)$ e $h \in \mathcal{C}(Y, Z)$.

$\left(A_{2}\right) 1_{Y} \circ f=f$ e $f \circ 1_{X}=f$, para todo morfismo $f \in \mathcal{C}(X, Y)$. 
Observação 1.1.2. ((SELICK, 2008), Chapter 1, Section 1, pg. 3) Quando a categoria é pressuposta escreveremos $f: X \longrightarrow Y$ para indicar que $f$ é um morfismo de $X$ para $Y$.

Definição 1.1.3. Se existem morfismos $f: X \longrightarrow Y$ e $g: Y \longrightarrow X$ tais que $g \circ f=1_{X}$ e $f \circ g=1_{Y}$ então $X$ e $Y$ são chamados isomorfos e $f$ e $g$ são chamados isomorfismos. Escreveremos $X \cong Y$, para expressar que $X$ é isomorfo a $Y$.

Exemplo 1.1.4. ((MACLANE, 1971), Chapter I, Section 2, pg. 12) São exemplos de categorias:

(i) Set denota a categoria cujos objetos são os conjuntos e cujos morfismos são todas as funções entre eles.

(ii) Grp denota a categoria cujos objetos são grupos e cujos morfismos são os homomorfismos de grupos.

(iii) Grp $A b$ ou $A b$ denota a categoria cujos objetos são grupos abelianos e cujos morfismos são homomorfismos de grupos.

(iv) $R-\bmod$ denota a categoria cujos objetos são $R$-módulos (à esquerda) e cujos morfismos são $R$-homomorfismos.

(vi) Top denota a categoria cujos objetos são espaços topológicos e cujos morfismos são funções contínuas.

(vii) $T o p_{i}$ denota a categoria cujos objetos são espaços topológicos não finitos e cujos morfismos são aplicações contínuas injetoras. Tal categoria é mencionada na Observação 2.1.11.

(vii) $T o p^{2}$ denota a categoria cujos objetos são pares espaços topológicos $(X, A)$, isto é, $X$ é um espaço topológico e $A \subset X$, e cujos morfismos são aplicações contínuas entre tais pares $f:(X, A) \longrightarrow(Y, B)$, ou seja, $f: X \longrightarrow Y$ é contínua e $f(A) \subset B$. Em particular, se $A=\left\{x_{0}\right\}$ e $B=\left\{f\left(x_{0}\right)\right\}$, temos a categoria cujos objetos são pares de espaços topológicos $\left(X, x_{0}\right)$ e cujos morfismos são aplicações contínuas entre tais pares $f:\left(X, x_{0}\right) \longrightarrow\left(Y, f\left(x_{0}\right)\right)$.

Observação 1.1.5. Uma categoria com exatamente um objeto, o qual denotaremos por * é similar a um monóide, isto é, um conjunto $M=\operatorname{Hom}(\star, \star)$ equipado com uma lei de composição associativa e de um elemento identidade. Desta maneira, um grupo pode ser considerado como uma categoria com um só objeto (vide (WEIBEL, 1994), Appendix A, Section A.1, pg. 418).

Definição 1.1.6. ((SELICK, 2008), Chapter 1, Section 1, Definition 1.1.2, pg. 3) Sejam $\mathcal{C}$ e $\mathcal{D}$ categorias. Um funtor covariante $F: \mathcal{C} \longrightarrow \mathcal{D}$ consiste de: 
$\left(F_{1}\right)$ um objeto $F(X)$ de $\operatorname{Obj} \mathcal{D}$ para cada objeto $X$ de $O b j \mathcal{C}$.

$\left(F_{2}\right)$ um morfismo $F(g) \in \mathcal{D}(F(X), F(Y))$, para cada morfismo $g \in \mathcal{C}(X, Y)$.

Tais objetos e morfismos satisfazem as seguintes condições:

$\left(F_{3}\right) F(f \circ g)=F(f) \circ F(g)$, para todo $g \in \mathcal{C}(X, Y)$ e $f \in \mathcal{C}(Y, Z)$.

$\left(F_{4}\right) F\left(1_{X}\right)=1_{F(X)}$, para todos os objetos $X$ de $\mathcal{C}$.

De maneira análoga, um functor contravariante é definido invertendo-se os objetos $F(X)$ e $F(Y)$ em $\left(F_{2}\right)$ e invertendo-se o ordem da composição em $\left(F_{3}\right)$.

Definição 1.1.7. Sejam categorias $\mathcal{C}$ e $\mathcal{D}$. Um funtor contravariante $F: \mathcal{C} \longrightarrow \mathcal{D}$ consiste:

$\left(F_{1}\right)$ de um objeto $F(X)$ de $O b j \mathcal{D}$, para cada objeto $X$ de $O b j \mathcal{C}$.

$\left(F_{2}\right)$ de um morfismo $F(g) \in \mathcal{D}(F(Y), F(X))$, para cada morfismo $g \in \mathcal{C}(X, Y)$.

Tais objetos e morfismos satisfazem as seguintes condições:

$\left(F_{3}\right) F(f \circ g)=F(g) \circ F(f)$, para todo $g \in \mathcal{C}(X, Y)$ e $f \in \mathcal{C}(Y, Z)$.

$\left(F_{4}\right) F\left(1_{X}\right)=1_{F(X)}$, para todos os objetos $X$ de $\mathcal{C}$.

Exemplo 1.1.8. São exemplos de funtores, para todo natural $k \geq 1$ :

$$
\begin{aligned}
F(-, k): \text { Top }_{i} & \longrightarrow \text { Top }_{i} \\
X & \mapsto F(X, k) \\
(f: X \longrightarrow Y) & \mapsto(F(f, k): F(X, k) \longrightarrow F(Y, k)),
\end{aligned}
$$

onde $F(X, k)$ é o espaço de configurações ordenado de $k$ pontos distintos de um espaço topológico $X, \forall k \geq 1$ (vide Definição 2.1.1).

$$
\begin{aligned}
S F(-, k): \text { Top }_{i} & \longrightarrow \text { Top }_{i} \\
X & \mapsto S F(X, k) \\
(f: X \longrightarrow Y) & \mapsto(S F(f, k): S F(X, k) \longrightarrow S F(Y, k))
\end{aligned}
$$

onde $S F(X, k)$ é o espaço de configurações não ordenado de $k$ pontos distintos de um espaço topológico $X, \forall k \geq 1$ (vide Definição 2.1.6)

Tais funtores são chamados functores de configurações (vide Observação 2.1.11). 
Definição 1.1.9. ((SELICK, 2008), Chapter 1, Section 1, Definition 1.1.3, pg. 3 e (MACLANE, 1971), Chapter I, Section 4, pg. 16).

Sejam $\mathcal{C}$ e $\mathcal{D}$ categorias e $F, G$ funtores de $\mathcal{C}$ em $\mathcal{D}$ :

$$
F, G: \mathcal{C} \longrightarrow \mathcal{D}
$$

Uma transformação natural $\eta: F \longrightarrow G$ é uma família de morfismos $\eta_{X}: F(X) \longrightarrow G(X)$ para cada objeto $X$ de $\mathcal{C}$ tal que o diagrama na Figura 1

Figura 1 - Transformação natural

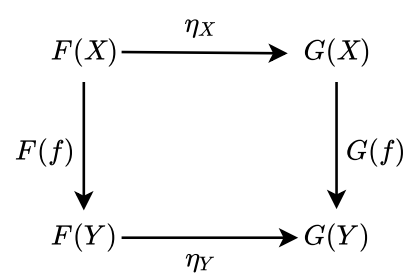

Fonte: Elaborada pelo autor.

é comutativo, para todo $f \in \mathcal{C}(X, Y)$ morfismo. Uma transformação natural $\eta$ tal que $\eta_{X}$ é um isomorfismo, para cada objeto $X$, é chamada uma equivalência natural ou um isomorfismo natural (em (WEIBEL, 1994), Appendix A, Section A.3, pg. 423).

Exemplo 1.1.10. Consideremos os funtores de configurações:

$$
F(-, k), F(-. r): \text { Top }_{i} \longrightarrow \text { Top, para } r<k
$$

Note que, neste caso, estamos considerando a categoria de chegada como sendo Top. A regra $\pi_{k, r}: F(-, k) \longrightarrow F(-, r)$ que associa para cada $X \in T o p_{i}$, a aplicação contínua $\pi_{k, r}: F(X, k) \longrightarrow F(X, r), \quad\left(x_{1}, \ldots, x_{k}\right) \mapsto\left(x_{1}, \ldots, x_{r}\right), \quad$ (vide Observação 3.1.24-(1)),

é uma transformação natural, mas não um isomorfismo natural, pois,

$$
\pi_{k, r}: F(X, k) \longrightarrow F(X, r), \quad\left(x_{1}, \ldots, x_{k}\right) \mapsto\left(x_{1}, \ldots, x_{r}\right)
$$

não é injetor.

Observação 1.1.11. ((WEIBEL, 1994), Appendix A, Section A.2, pg. 421) Um funtor $F: \mathcal{C} \longrightarrow \mathcal{D}$ de uma categoria $\mathcal{C}$ em uma categoria $\mathcal{D}$, induz uma aplicação:

$$
H_{\mathcal{C}}(X, Y) \longrightarrow \operatorname{Hom}_{\mathcal{D}}(F(X), F(Y)),
$$

para cada $X, Y \in \mathcal{C}$. Se $G: \mathcal{D} \longrightarrow \mathcal{E}$ é outro funtor, a composição

$$
G F: \mathcal{C} \longrightarrow \mathcal{E}
$$


é definida por

$$
X \mapsto(G F)(X):=G(F(X)) \text { e } f \mapsto(G F)(f):=G(F(f))
$$

O funtor identidade $I d_{\mathcal{C}}: \mathcal{C} \longrightarrow \mathcal{C}$ é a regra fixando todos os objetos e morfismos, isto é, $I d_{\mathcal{C}}(X):=X$ e $I d_{\mathcal{C}}(f):=f$. Assim, para um funtor $F: \mathcal{C} \longrightarrow \mathcal{D}$ temos

$$
F \circ I d_{\mathcal{C}}=F \text { e } I d_{\mathcal{D}} \circ F=F
$$

Observação 1.1.12. Um funtor $F: \mathcal{C} \longrightarrow \mathcal{D}$ entre duas categorias com um só objeto é similar a um homomorfismo de monóides.

Definição 1.1.13. ((MACLANE, 1971), Chapter I, Section 3, pg. 14) Um funtor que simplesmente esquece parte da estrutura de um objeto algébrico é comumente chamado um funtor esquecimento ${ }^{1}$ e será denotado pela letra $E$. Como exemplos, existem: um funtor esquecimento de $R$-mod para $A b$, o qual esquece a estrutura de $R$-módulo; um funtor de esquecimento de $A b$ a $S e t$, o qual esquece a estrutura de grupo; e sua composição de $R-\bmod$ para Set.

Definição 1.1.14. (WEIBEL, 1994), Appendix A, Section A.2, pg. 421) Um funtor $F: \mathcal{C} \longrightarrow \mathcal{D}$ é chamado fiel ${ }^{2}$ se as aplicações

$$
\operatorname{Hom}_{\mathcal{C}}\left(X, X^{\prime}\right) \longrightarrow \operatorname{Hom}_{\mathcal{D}}\left(F(X), F\left(X^{\prime}\right)\right)
$$

são todas injetivas, ou seja, se $f, g$ são morfismos distintos de $X$ em $X^{\prime}$, então $F(f) \neq$ $F(g)$. Funtores esquecimento são funtores fiéis. Uma categoria $\mathcal{C}$ com um funtor fiel $E: \mathcal{C} \longrightarrow$ Set é chamada uma categoria concreta. Em uma categoria concreta, os morfismos são completamente determinados por seus efeitos sobre os conjuntos subjacentes.

Exemplo 1.1.15. $R-\bmod$ e $A b$ são exemplos de categorias concretas.

Exemplo 1.1.16. $F(-, k): T o p_{i} \longrightarrow T o p_{i}$ e $S F(-, k): T o p_{i} \longrightarrow T o p_{i}$ são funtores fiéis.

Definição 1.1.17. ((WEIBEL, 1994), Appendix A, Section A.2, pg. 422) Uma subcategoria $\mathcal{B}$ de uma categoria $\mathcal{C}$ é uma coleção de alguns dos objetos de $\mathcal{C}$ e alguns dos morfismos de $\mathcal{C}$ tais que os morfismos de $\mathcal{B}$ são fechados sob composições, incluindo $1_{X}$, para todo objeto $X$ em $\operatorname{Obj} \mathcal{B}$. Note que uma subcategoria é uma categoria e existe um functor, chamado functor inclusão:

$$
\begin{aligned}
i: \mathcal{B} \hookrightarrow \mathcal{C}, \quad X \longmapsto i(X):=X, \quad f & \longmapsto i(f):=f, \\
\operatorname{Hom}_{\mathcal{B}}\left(X, X^{\prime}\right) \longrightarrow \operatorname{Hom}_{\mathcal{C}}\left(X, X^{\prime}\right) \quad f & \longmapsto f,
\end{aligned}
$$

o qual é fiel por definição. Uma subcategoria $\mathcal{B}$ na qual $\operatorname{Hom}_{\mathcal{B}}\left(X, X^{\prime}\right)=\operatorname{Hom}_{\mathcal{C}}\left(X, X^{\prime}\right)$, para cada $X, X^{\prime}$ em $\mathcal{B}$ é chamada subcategoria completa.

1 Ou, o mesmo termo em inglês, Forgetful functor.

2 Ou, o mesmo termo em inglês, faithful. 
Exemplo 1.1.18. Top $p_{i}$ é uma subcategoria de Top, a qual não é completa, pois aplicações constantes não são morfismos em $T_{o p}$.

Definição 1.1.19. ((WEIBEL, 1994), Appendix A, Section A.2, pg. 422) Um funtor $F: \mathcal{C} \longrightarrow \mathcal{D}$ é completo se as aplicações

$$
\operatorname{Hom}_{\mathcal{C}}\left(X, X^{\prime}\right) \longrightarrow \operatorname{Hom}_{\mathcal{D}}\left(F(X), F\left(X^{\prime}\right)\right)
$$

são todas sobrejetoras, ou seja, todo morfismo $g: F(X) \rightarrow F\left(X^{\prime}\right)$ em $\mathcal{D}$ é da forma $g=F(f)$, para algum morfismo $f: X \rightarrow X^{\prime}$ em $\mathcal{C}$. Um funtor que é completo e fiel é chamado completamente fiel.

Exemplo 1.1.20. A inclusão de uma subcategoria completa é completamente fiel.

Definição 1.1.21. ((WEIBEL, 1994), Appendix A, Section A.2, pg. 423) Um funtor $F: \mathcal{C} \longrightarrow \mathcal{D}$ é chamado uma equivalência de categorias se existem um funtor $G: \mathcal{D} \longrightarrow \mathcal{C}$ e isomorfismos naturais $1_{\mathcal{C}} \cong G F$ e $1_{\mathcal{D}} \cong F G$.

\subsection{Topologia compacta aberta}

Usando ((AGUILAR; GITLER; PRIETO, 2002), Chapter 1, section 1.1, Definition 1.1.1, pg. 1), recordemos que se $X$ e $Y$ são conjuntos, $Y^{X}$ denota o conjunto de todas as funções $f: X \rightarrow Y$. Podemos interpretar $Y^{X}$ como o produto cartesiano $\prod_{x \in X} Y_{x}$, onde $Y_{x}=Y, \forall x \in X$. Quando $Y$ é um espaço topológico, uma topologia canônica sobre $Y^{X}=\prod_{x \in X} Y_{x}$ é a topologia produto. Uma sub-base para esta topologia é formada pela família de conjuntos $U^{x}=\left\{f \in Y^{X} ; f(x) \in U\right\}$, onde $x \in X$ e $U$ é um conjunto aberto em $Y$.

Agora, supondo que $X$ e $Y$ são espaços topológicos, podemos considerar o subconjunto $\operatorname{Map}(X, Y) \subset Y^{X}$ consistindo de todas as funções contínuas de $X$ em $Y$. Introduziremos uma topologia canônica sobre $\operatorname{Map}(X, Y)$, chamada topologia compacta-aberta, a qual é uma topologia que leva em consideração tanto a topologia de $X$ quanto a topologia de $Y$ e que generaliza a topologia produto.

Definição 1.2.1. ((AGUILAR; GITLER; PRIETO, 2002), Chapter 1, section 1.2, Definition 1.2.1, pg. 2) A topologia compacta-aberta, denotada $\tau_{c a}$, sobre $M a p(X, Y)$ tem como sub-base a coleção dos conjuntos

$(C, U):=\{f \in \operatorname{Map}(X, Y): f(C) \subset U\}$, onde $C \subset X$ é compacto e $U$ é aberto em $Y$.

Ou seja,

$$
\tau_{c a}=\left\{A \subset M a p(X, Y): A=\bigcup\left(\bigcap_{\text {finita }}(C, U)\right)\right\}
$$


Observação 1.2.2. Se $\tau$ é uma topologia sobre $\operatorname{Map}(X, Y)$ usaremos a notacão $\operatorname{Map} \tau(X, Y)$ para o correspondente espaço topológico. Assim, usaremos a notação

$$
\operatorname{Map}_{c a}(X, Y)=\left(M a p(X, Y), \tau_{c a}\right)
$$

quando $\tau=c a$ for a topologia compacta aberta.

Proposição 1.2.3. Se $B$ é um subespaço de $Y$, então $M a p_{c a}(X, B)$ é um subespaço de $\operatorname{Map}_{c a}(X, Y)$.

Demonstração. Seja $(C, U)$ um aberto sub-básico para $M a p_{c a}(X, B)$. Vejamos que

$$
(C, U)=W \cap M a p(X, B), \text { para algum aberto } W \text { de } M a p_{c a}(X, Y) .
$$

De fato, seja $V$ aberto em $Y$ tal que $U=V \cap B$. Consideremos o aberto sub-básico $W=(C, V)$ de $\operatorname{Map}_{c a}(X, Y)$. Mostremos que $(C, U)=W \cap \operatorname{Map}(X, B)$.

$$
\begin{aligned}
f \in(C, U) & \Longleftrightarrow f \in \operatorname{Map}(X, B) \text { e } f(C) \subseteq U \\
& \Longleftrightarrow f \in \operatorname{Map}(X, B) \text { e } f(C) \subseteq V \\
& \Longleftrightarrow f \in \operatorname{Map}(X, B) \text { e } f \in(C, V) \\
& \Longleftrightarrow f \in W \cap M a p(X, B) .
\end{aligned}
$$

Reciprocamente, seja $(C, V)$ aberto sub-básico de $M a p_{c a}(X, Y)$. Note que

$$
(C, V) \cap \operatorname{Map}(X, B)=(C, V \cap B)
$$

o qual é um aberto sub-básico de $\operatorname{Map}(X, B)$.

Definição 1.2.4. ((AGUILAR; GITLER; PRIETO, 2002), Chapter 1, Section 1.1, pg. 2)

A aplicação $e: \operatorname{Map}(X, Y) \times X \rightarrow Y$

$$
(f, x) \quad \longmapsto e(f, x)=f(x), \forall f \in \operatorname{Map}(X, Y), \forall x \in X,
$$

é chamada aplicação avaliação.

Definição 1.2.5. ((AGUILAR; GITLER; PRIETO, 2002), Chapter 1, Section 1.1, Definition 1.1.3, pg. 2) Uma topologia $\tau$ sobre $\operatorname{Map}(X, Y)$ é chamada admissível se a aplicação avaliação $e$ for contínua com respeito a essa topologia $\tau$, ou seja,

$$
e: \operatorname{Map}_{\tau}(X, Y) \times X \longrightarrow Y \text { é contínua }
$$

Definição 1.2.6. ((LEE, 2010), Chapter 4, pg. 104) Um subconjunto $A$ de um espaço topológico $X$ é chamado relativamente compacto $^{3}$ se seu fecho $\bar{A}$ em $X$ for compacto.

3 Ou pré-compacto. 
Definição 1.2.7. (LEE, 2010), Chapter 4, pg. 104) Um espaço $X$ é chamado localmente compacto se para cada ponto $q \in X$ existe um subconjunto compacto de $X$ contendo uma vizinhança de $p$.

Exemplo 1.2.8. Todo espaço compacto é localmente compacto. O espaço Euclideano $\mathbb{R}^{n}$ é localmente compacto, mas não é compacto.

Considerando $X$ espaço Hausdorff a propriedade de localmente compacto, é muito mais útil.

Proposição 1.2.9. ((LEE, 2010), Chapter 4, Proposition 4.63, pg. 104) Seja $X$ um espaço Hausdorff. Os seguentes enunciados são equivalentes:

1. $X$ é localmente compacto.

2. Cada ponto de $X$ tem uma vizinhança pré-compacta.

3. $X$ tem uma base de subconjuntos abertos pré-compactos.

Definição 1.2.10. Para um espaço topológico $X$, denotaremos por $T o p(X)$ o conjunto de todos os homeomorfismos $\phi: X \rightarrow X$, o qual é um espaço topológico munido da topologia compacta aberta.

Proposição 1.2.11. Seja $X$ um espaço localmente compacto Hausdorff ${ }^{4}$.

(1) A topologia compacta-aberta $\tau_{c a}$ é admissível (vide (AGUILAR; GITLER; PRIETO, 2002), Chapter 1, Section 1.2, Proposition 1.2.3, pg. 3).

(2) A aplicação $\varphi: \operatorname{Top}(X) \times \operatorname{Top}(X) \longrightarrow \operatorname{Top}(X), \quad \varphi(g, f):=f \circ g$ é contínua.

Demonstração. (1) Mostremos que a aplicação avaliação $e: \operatorname{Map}_{c a}(X, Y) \times X \longrightarrow Y$ é contínua . Sejam $U \subseteq Y$ um aberto e $(f, x) \in e^{-1}(U)$, com $f \in \operatorname{Map}(X, Y)$ e $x \in X$.

Como $f(x)=e(f, x) \in U$, então $x \in f^{-1}(U)$. Pela continuidade de $f$, temos que $f^{-1}(U)$ é um aberto em $X$ contendo $x$. Assim, existe uma vizinhança aberta $W$ de $x$ em $X$ com $x \in W \subseteq f^{-1}(U) \subseteq X$.

Sendo $X$ localmente compacto Hausdorff, existe uma vizinhança aberta $V$ de $x$ em $X$, tal que $x \in V \subseteq \bar{V} \subseteq W$, com $\bar{V}$ compacto em $X$ (vide Proposição 1.2.9-(3)).

O produto $(\bar{V}, U) \times V$ é um aberto em $\operatorname{Map}_{c a}(X, Y) \times X$ contendo $(f, x)$. Assim, é suficiente provar que

$$
(\bar{V}, U) \times V \subseteq e^{-1}(U)
$$

4 Por exemplo, o intervalo unitário $I=[0,1]$ 
De fato, se $(g, y) \in(\bar{V}, U) \times V$, então

$$
g(\bar{V}) \subseteq U \text { e } y \in V
$$

Então,

$$
e(g, y)=g(y) \in g(V) \subseteq g(\bar{V}) \subseteq U .
$$

Portanto, $(g, y) \in e^{-1}(U)$.

(2) Mostremos que $\varphi: \operatorname{Top}(X) \times \operatorname{Top}(X) \longrightarrow \operatorname{Top}(X)$ definida por $\varphi(g, f):=f \circ g$ é contínua. Sejam $(C, U)$ um aberto sub-básico em $\operatorname{Top}(X)$ e $(g, f) \in \varphi^{-1}((C, U))$. Então,

$$
f \circ g=\varphi(g, f) \in(C, U) \text { e } \underbrace{g(C)}_{\text {compacto }} \subseteq \underbrace{f^{-1}(U)}_{\text {aberto }} .
$$

Como $X$ é localmente compacto Hausdorff, existe um conjunto $V$ aberto em $X$ tal que

$$
g(C) \subseteq V \subseteq \bar{V} \subseteq f^{-1}(U), \text { com } \bar{V} \text { compacto. }
$$

Consideremos $(C, V)$ e $(\bar{V}, U)$ abertos sub-básicos em Top $(X)$. Afirmamos que:

$$
(g, f) \in(C, V) \times(\bar{V}, U) \subseteq \varphi^{-1}((C, U)),
$$

De fato, para $(h, l) \in(C, V) \times(\bar{V}, U)$, temos que $h(C) \subseteq V$ e $l(\bar{V}) \subseteq U$. Assim,

$$
\varphi(h, l)(C)=(l \circ h)(C) \subseteq l(V) \subseteq l(\bar{V}) \subseteq U .
$$

Portanto, $\varphi^{-1}((C, U))$ é aberto em $\operatorname{Top}(X) \times \operatorname{Top}(X)$.

Proposição 1.2.12. ((AGUILAR; GITLER; PRIETO, 2002), Chapter 1, section 1.3, Proposition 1.3.4, pg. 5) Seja $X$ um espaço compacto Hausdorff. Então,

$$
\psi: \operatorname{Top}(X) \longrightarrow \operatorname{Top}(X), \quad \psi(f):=f^{-1} \text { é contínua. }
$$

Demonstração. Sejam $(C, U)$ um aberto sub-básico em Top $(X)$ e $f \in \psi^{-1}((C, U))$. Então,

$$
f^{-1}=\psi(f) \in(C, U) \text { e } f^{-1}(C) \subseteq U, \quad \log \mathrm{O} ; C \subseteq f(U) .
$$

Considerando complementares e usando o fato que $f$ é uma bijeção, temos:

$$
f(X-U)=X-f(U) \subseteq X-C .
$$

Assim, $(X-U, X-C)$ é um aberto sub-básico em Top $(X)$ contendo $f$ e

$$
(X-U, X-C) \subseteq \psi^{-1}((C, U))
$$

De fato, para $g \in(X-U, X-C)$, temos

$$
X-g(U)=g(X-U) \subseteq X-C, \quad \log 0 \quad C \subseteq g(U) \text { e } g^{-1}(C):=\psi(g)(C) \subseteq U .
$$

Portanto, $\psi$ é contínua. 
Corolário 1.2.13. Seja $X$ um espaço compacto Hausdorff. O grupo Top $(X)$, dos homeomorfismos de $X$, munido com a operação de composição de funções e equipado com a topologia compacta aberta é um grupo topológico.

Observação 1.2.14. De agora em diante, sempre consideraremos a topologia compacta aberta sobre $\operatorname{Map}(X, Y)$ e denotaremos $\operatorname{Map}_{c a}(X, Y)$ simplesmente por $\operatorname{Map}(X, Y)$.

Proposição 1.2.15. Sejam $X, Y, Z$ espaços topológicos. Então, existe uma aplicação $\varphi: \operatorname{Map}(X \times Y, Z) \longrightarrow \operatorname{Map}(X, \operatorname{Map}(Y, Z))$, definida por

$$
\varphi(f)(x)(y):=f(x, y), \forall f \in M a p(X \times Y, Z), \quad \forall x \in X \text { e } \forall y \in Y .
$$

A aplicação $\varphi$ é chamada aplicação associação.

Demonstração. Mostremos que $\varphi(f)(x): Y \longrightarrow Z$ é contínua. De fato, ela é dada pela composição de funções contínuas:

$$
\varphi(f)(x)=f \circ i_{x}, \text { onde } i_{x}: Y \longrightarrow X \times Y, i_{x}(y)=(x, y), \quad \forall y \in Y .
$$

$\operatorname{Assim} \varphi(f)(x) \in \operatorname{Map}(Y, Z)$. Além disso, temos também que

$$
\varphi(f): X \longrightarrow \operatorname{Map}(Y, Z)
$$

é contínua. De fato, seja $(C, U)$ um aberto sub-básico de $M a p(Y, Z)$, com $C$ compacto em $Y$ e $U$ aberto em $Z$ e mostremos que $\varphi^{-1}(f)((C, U))$ é aberto em $X$. Para isto, seja $x \in \varphi^{-1}(f)((C, U))$. Para todo $c \in C$,

$$
f(x, c):=\varphi(f)(x)(c) \in U, \quad \log 0(x, c) \in f^{-1}(U) \text { aberto em } X \times Y .
$$

Então, existem vizinhanças abertas $W_{c}$ de $x$ e $V_{c}$ de $c$ tais que

$$
(x, c) \in W_{c} \times V_{c} \subseteq f^{-1}(U) \text { o que implica } f(x, c) \in f\left(W_{c} \times V_{c}\right) \subseteq U .
$$

A coleção

$$
\left\{V_{c}\right\}_{c \in C}
$$

é uma cobertura aberta do compacto $C$, assim existe uma sub-coleção finita

$$
\left\{V_{c_{1}}, V_{c_{2}}, \cdots, V_{c_{n}}\right\}
$$

que ainda cobre $C$. Sejam $W_{c_{1}}, W_{c_{2}}, \cdots, W_{c_{n}}$ as vizinhanças que correspondem a $V_{c_{i}}$, para cada $1 \leq i \leq n$. Consideremos

$$
W=\bigcap_{i=1}^{n} W_{c_{i}}
$$

vizinhança aberta de $x$ em $X$ e mostremos que

$$
x \in W \subseteq \varphi^{-1}(f)((C, U)) .
$$

De fato, se $z \in W$ e $c \in C$, então $\varphi(f)(z)(c)=f(z, c)$, mas $c \in V_{c_{i}}$, para algum $i$ e $z \in W_{c_{i}}$, $\operatorname{logo} f(z, c) \in U$. Assim, $\varphi(f)$ é contínua e, portanto, $\varphi$ é bem definida. 
Observação 1.2.16. Em (AGUILAR; GITLER; PRIETO, 2002), Chapter 1, section 1.3, Proposition 1.3.1, pg. 4, os autores mostram ainda que a aplicação associação $\varphi$ dada na Proposição 1.2.15 é uma equivalência de conjuntos, ou seja, existe

$$
\psi: \operatorname{Map}(X, \operatorname{Map}(Y, Z)) \rightarrow \operatorname{Map}(X \times Y, Z)
$$

uma inversa para $\varphi$. Para mostrar a boa definição de $\psi$ é necessário considerar a hipótese de $Y$ ser localmente compacto Hausdorff.

Proposição 1.2.17. ((AGUILAR; GITLER; PRIETO, 2002), Chapter 1, section 1.3, Proposition 1.3.2, pg. 5) Sejam $X, Y, Z$ espaços topológicos, com $X$ Hausdorff. Então,

$$
\varphi: M a p(X \times Y, Z) \longrightarrow M a p(X, M a p(Y, Z))
$$

é contínua.

Demonstração. Seja $(C, U)$ um aberto sub-básico de $\operatorname{Map}(X, \operatorname{Map}(Y, Z)) \operatorname{com} C \subseteq X$ compacto e $U=(K, V)$ com $K$ compacto em $Y$ e $V$ aberto em $Z$.

$$
\begin{aligned}
& \varphi^{-1}((C, U))=\{f \in \operatorname{Map}(X \times Y, Z): \varphi(f) \in(C, U)\} \\
& =\{f \in \operatorname{Map}(X \times Y, Z): \varphi(f)(C) \subseteq(K, V)\} \\
& =\{f \in \operatorname{Map}(X \times Y, Z): \varphi(f)(C)(K) \subseteq V\} \\
& =\{f \in \operatorname{Map}(X \times Y, Z): f(C \times K) \subseteq V\} \\
& =(C \times K, V)
\end{aligned}
$$

Logo, $\varphi$ é continua.

Observação 1.2.18. Em (AGUILAR; GITLER; PRIETO, 2002), Chapter 1, section 1.3, Proposition 1.3.2, pg. 4, os autores mostram ainda que a aplicação associação $\varphi$ dada na Proposição 1.2.17 é um homeorfismo. Para isto, é necessário considerar a hipótese adicional de que $Y$ é localmente compacto Hausdorff.

Proposição 1.2.19. Seja $I=[0,1]$. A aplicação concatenação

$$
\begin{aligned}
\psi: \operatorname{Map}(I, X) \times \operatorname{Map}(I, X) & \longrightarrow \operatorname{Map}(I, X) \\
(\alpha, \beta) & \longmapsto \psi(\alpha, \beta):=\alpha * \beta, \quad \forall \alpha, \beta \in \operatorname{Map}(I, X),
\end{aligned}
$$

é contínua, onde $\alpha(1)=\beta(0)$ e $\alpha * \beta: I \longrightarrow X$ é a concatenacão dos caminhos $\alpha$ e $\beta$, i.e.,

$$
(\alpha * \beta)(t)= \begin{cases}\alpha(2 t), & 0 \leq t \leq \frac{1}{2} \\ \beta(2 t-1), & \frac{1}{2} \leq t \leq 1\end{cases}
$$


Demonstração. Seja $(C, U)$ um aberto sub-básico em $\operatorname{Map}(I, X)$, com $C \subset I$ compacto e $U$ aberto em $X$. Vejamos que $\psi^{-1}(C, U)$ é aberto em $\operatorname{Map}(I, X) \times \operatorname{Map}(I, X)$. De fato,

$$
\begin{aligned}
\psi^{-1}(C, U) & =\{(\alpha, \beta): \psi(\alpha, \beta) \in(C, U)\} \\
& =\{(\alpha, \beta): \alpha * \beta \in(C, U)\} \\
& =\{(\alpha, \beta): \alpha * \beta(C) \subseteq U\} \\
& =\left(C \cap\left[0, \frac{1}{2}\right], U\right) \times\left(C \cap\left[\frac{1}{2}, 1\right], U\right)
\end{aligned}
$$

Assim, $\psi^{-1}(C, U)$ é aberto em $\operatorname{Map}(I, X) \times \operatorname{Map}(I, X)$.

\subsection{Grupos de transformações ou $G$-ações}

Nesta seção, introduzimos uma série de definições e propriedades fundamentais para grupos de transformações (KAWAKUBO, 1991). Consideramos também grupos de transformações com estruturas adicionais, como a topologia, a estrutura $C^{\infty}$ e uma estrutura algébrica.

Definição 1.3.1. ((KAWAKUBO, 1991), Chapter 1, Section 1.1, Definition 1.1, pg. 2) Sejam $G$ um grupo com elemento identidade e e $X$ um conjunto. Uma $G$-ação à esquerda é uma aplicação

$$
\begin{aligned}
\varphi: G \times X & \longrightarrow X \\
(g, x) & \mapsto \varphi(g, x):=g \cdot x
\end{aligned}
$$

satisfazendo as seguintes condições:

(1) $e \cdot x=x, \forall x \in X$, ou seja, o elemento identidade $e$ age "trivialmente".

(2) $g_{2} \cdot\left(g_{1} \cdot x\right)=\left(g_{2} g_{1}\right) \cdot x, \quad \forall g_{1}, g_{2} \in G, \forall x \in X$.

A tripla $(X, G, \varphi)$ é chamado um grupo de transformações ou uma $G$-ação sobre $X$ e $X$ é chamado um $G$-conjunto.

Observação 1.3.2. ((KAWAKUBO, 1991), Chapter 1, Section 1.1, pg. 3) Para cada $g \in G$ a aplicação $\varphi_{g}: X \longrightarrow X$ definida por $\varphi_{g}(x):=\varphi(g, x) . \varphi_{g}$ é chamada uma transformação por $g$. Das condições (1) e (2), segue que $\varphi_{g}$ é uma bijeção com inversa $\varphi_{g^{-1}}$

Exemplo 1.3.3. ((KAWAKUBO, 1991), Chapter 1, Section 1.1, Example 9, pg. 3) Sejam $G$ um grupo topológico, $X$ um espaço topológico e $\varphi: G \times X \longrightarrow X$ uma $G$-ação contínua. Para cada $g \in G, \varphi_{g}: X \rightarrow X$ é um homeomorfismo (vide (KAWAKUBO, 1991), Lemma 1.29 , pg. 24). A tripla $(X, G, \varphi)$ é chamada um grupo de transformações topológico e $X$ é chamado um $G$-espaço. 
Observação 1.3.4. O caso citado no Exemplo 1.3 .3 será usado para definir o espaço de configurações não ordenado, no Capítulo 2.

Exemplo 1.3.5. Sejam $G$ um grupo e $H$ um subgrupo de $G$. Denotemos por $X=G / H$ o conjunto das classes laterias $g H$ de $H$ em $G$. Então, a aplicação:

$$
\varphi: G \times G / H \longrightarrow G / H
$$

definida por $\varphi\left(g^{\prime}, g H\right)=g^{\prime}(g H)=\left(g g^{\prime}\right) H$ define uma $G$-ação.

Proposição 1.3.6. Se $G$ é um grupo discreto, ou seja, $G$ é um grupo munido da topologia discreta, e se $X$ é um espaço topológico qualquer, então $\varphi: G \times X \longrightarrow X$ é contínua se, e somente se, $\varphi_{g}: X \longrightarrow X$ é contínua, $\forall g \in G$.

Demonstração. Se $\varphi: G \times X \longrightarrow X$ é contínua, então $\varphi_{g}: X \longrightarrow X$ é contínua, para todo $g \in G$, pois é a restrição a $X$ da aplicação contínua $\varphi$.

Reciprocamente, dado $g \in G$, suponhamos que $\varphi_{g}: X \longrightarrow X$ seja contínua. Sejam $U \subset X$ um aberto e $(g, x) \in \varphi^{-1}(U)$. Então, $\varphi(g, x):=\varphi_{g}(x) \in U$, logo $x \in \varphi_{g}^{-1}(U)$. Como por hipótese $\varphi_{g}^{-1}(U)$ é um aberto em $X$ contendo o ponto $x$, existe $V \subseteq X$ aberto tal que $x \in V \subseteq \varphi_{g}^{-1}(U)$. Assim, $(g, x) \in\{g\} \times V \subseteq \varphi^{-1}(U)$, com $\{g\} \times V$ aberto em $G \times X$.

Definição 1.3.7. ((KAWAKUBO, 1991), Chapter 1, Section 1.1, pg. 4) Seja $(X, G, \varphi)$ um grupo de transformações.

1. Para cada $x \in X$, o conjunto

$$
G x:=G(x):=\{g x \in X: \quad g \in G\}
$$

é chamado a órbita de $x$.

2. Para cada $x \in X$, o conjunto

$$
G_{x}:=\{g \in G: \quad g x=x\}
$$

dos elementos de $G$ fixando $x$ é chamado o grupo de isotropia em $x$.

3. O conjunto

$$
X^{G}:=\left\{x \in X: G_{x}=G\right\}
$$

é chamado o conjunto dos pontos fixos.

As propriedades fundamentais dos conjuntos na Definição 1.3.7 são apresentadas a seguir.

Proposição 1.3.8. ((KAWAKUBO, 1991), Chapter 1, Section 1.1, Propositions 1.1, 1.2 e $1.3, \mathrm{pg} .4$ e 5$)$ 
1. O grupo de isotropia $G_{x}$ é um subgrupo de $G$.

2. Para $x, y \in X$, ou $G(x)=G(y)$ ou $G(x) \cap G(y)=\emptyset$.

3. Para qualquer $g \in G$ e $x \in X$, temos

$$
G_{g x}=g G_{x} g^{-1}
$$

Observação 1.3.9. Seja $\varphi: G \times X \longrightarrow X$ uma $G$-ação contínua. Segue do Exemplo 1.3.3, que a correspondência $g \mapsto \varphi_{g}$ define uma aplicação

$$
\rho: G \longrightarrow T o p(X), \quad \rho(g):=\varphi_{g}
$$

Embora Top $(X)$ não seja necessariamente um grupo topológico, ele é um grupo, munido da operação de composição de funções, assim como um espaço topológico, munido da topologia compacto-aberta (vide Definição 1.2.10). A aplicação $\rho$ definida em (1.3.1) é um homomorfismo de grupos, chamado uma representação para $X$ (vide (KAWAKUBO, 1991), Chapter 1, Section 1.4, Lemma 1.30, pg. 24).

Reciprocamente, dado um homomorfismo $\rho: G \longrightarrow \operatorname{Top}(X)$, definimos $\varphi: G \times X \longrightarrow X$ por $\varphi(g, x):=\rho(g)(x)$. Então, $\varphi$ é uma $G$-ação no sentido da Definição 1.3 .1 (vide(KAWAKUBO, 1991), Chapter 1, Section 1.4, Lemma 1.31, pg. 25). O kernel do homomorfismo $\rho$ é um subgrupo normal de $G$, chamado o kernel da ação $\varphi$. Além disso, $\operatorname{Ker}(\rho)=\bigcap_{x \in X} G_{x}$ (vide (KAWAKUBO, 1991), Chapter 1, Section 1.4, Lemmas 1.34 e 1.35, pg. 25).

Definição 1.3.10. ((KAWAKUBO, 1991), Chapter 1, Section 1.1, pg. 6) Um grupo de transformações $(X, G, \varphi)$ é chamado:

1. trivial, se $G_{x}=G, \forall x \in X$, ou seja, $g x=x, \forall g \in G$ e $\forall x \in X$, isto é, o homomorfismo $\rho: G \longrightarrow \operatorname{Top}(X)$ é trivial.

2. livre, se $G_{x}=\{e\}, \forall x \in X$. Neste caso, dizemos que $G$ age livremente sobre $X$.

3. semi-livre, se $G_{x}=G$ ou $G_{x}=\{e\}, \forall x \in X$.

4. fiel ou efetiva, se o homomorfismo $\rho: G \longrightarrow T o p(X)$ é injetor, ou seja, $g x=x$, $\forall x \in X$ implica $g=e$.

5. transitivo, se existe exatamente uma órbita, ou seja, para quaisquer $x, y \in X$, existe $g \in G$ tal que $g x=y$;

6. $m$-transitivo, se $X$ possui pelo menos $m$-elementos e, para quaisquer $x_{1}, \ldots, x_{m}$ e $y_{1}, \ldots, y_{m}$ pontos distintos de $X$, existe $g \in G$ tal que $g x_{i}=y_{i}, \forall 1 \leq i \leq m$.

Observação 1.3.11. Toda ação livre é efetiva. 
Observação 1.3.12. O conceito de ação efetiva é usada por Steenrod na definição de fibrado (vide (STEENROD, 1951), pg. 7).

Definição 1.3.13. ((DIECK, 2008), pg. 64; (HATCHER, 2002), Chapter 1, Section 1.3, pg. 72) Seja $(X, G, \varphi)$ um grupo de transformações topológico. Dizemos que a ação de $G$ sobre $X$ é propriamente descontínua se, para cada $x \in X$, existe uma vizinhança aberta $V$ de $x$ tal que

$$
(g V) \cap\left(g^{\prime} V\right)=\emptyset, \forall g, g^{\prime} \in G, \text { com } g \neq g^{\prime}
$$

ou, equivalentemente,

$$
(g V) \cap V=\emptyset, \quad \forall g \in G, \text { com } g \neq e,
$$

onde $g V:=\{g x: x \in V\}$.

Observação 1.3.14. Toda ação propriamente descontínua é livre (vide (DIECK, 2008), pg. 64).

Definição 1.3.15. ((KAWAKUBO, 1991), Chapter 1, Section 1.6, Definition 1.3, pg. 36) Para um $G$-espaço $X$, definimos uma relação de equivalência $\sim$ sobre $X$ como segue:

$$
x \sim y \text { se, e somente se, existe um elemento } g \in G \text { com } y=g x \text {. }
$$

Denotaremos o espaço quociente $X / \sim$ por $X / G$, ou seja,

$$
X / G=\{G(x): x \in X\}
$$

é o conjunto cujos elementos são as órbitas $G(x)=\{g x: g \in G\}$ de $G$ sobre $X$. $X / G$ é um espaço topológico munido da topologia quociente co-induzida pela projeção natural $q: X \longrightarrow X / G$, chamado o espaço de órbitas de $X$.

Proposição 1.3.16. (Vide (BREDON, 1976). Teorema 3.1, pg. 38) Seja $X$ um $G$-espaço Hausdorff com $G$ compacto. Então:

(a) $X / G$ é Hausdorff.

(b) $q: X \longrightarrow X / G$ é fechada

(c) $q: X \longrightarrow X / G$ é uma aplicação própria ${ }^{5}$

(d) $X$ é compacto se, e somente se $X / G$ é compacto.

Lema 1.3.17. Se $X$ é um $G$-espaço, então $q: X \longrightarrow X / G$ é uma aplicação aberta.

$5 \quad$ Uma função contínua $f: X \rightarrow Y$ é própria se para todo compacto $K \subset Y$, temos $f^{-1}(K) \subset X$ compacto. 
Demonstração. Note que para qualquer subconjunto $U$ de $X$ :

$$
q^{-1}(q(U))=\bigcup_{g \in G} g U
$$

onde $g U=\{g x: x \in U\}$. Em particular, se $U$ for aberto em $X$, como $\varphi_{g}: X \rightarrow X$ é um homeomorfismo e $\varphi_{g}(U)=g U$, segue que $g U$ é aberto em $X$, o que implica $q^{-1}(q(U))$ aberto em $X$. Portanto, $q(U)$ é aberto em $X / G$.

Proposição 1.3.18. ((HATCHER, 2002), Chapter 1, Section 1.3, Proposition 1.40-(a), pg. 72) Seja $X$ um $G$-espaço. Se a ação de $G$ sobre $X$ é propriamente descontínua, então $q: X \longrightarrow X / G$ é um recobrimento.

Proposição 1.3.19. Sejam $X$ um espaço de Hausdorff e $G$ um grupo finito. Se a ação de $G$ sobre $X$ é livre, então ela também é propriamente descontínua.

Demonstração. Seja $G=\left\{g_{0}, g_{1}, \ldots, g_{n}\right\}$, onde $g_{0}=e$. Dado um ponto $x \in X$, como a ação de $G$ sobre $X$ é livre, então $G_{x}=\{e\}$. Logo

$$
g_{i} x \neq x, \quad \forall i \in\{1, \ldots, n\} .
$$

Mas como $X$ é Hausdorff, existem vizinhanças abertas $U_{0}, U_{1}, \cdots, U_{n}$ de $x, g_{1} x, \ldots, g_{n} x$, respectivamente, tais que $U_{0} \cap U_{j}=\emptyset$, para todo $j=1, \ldots, n$. Consideremos o conjunto

$$
U:=\bigcap_{j=0}^{n} g_{j}{ }^{-1} U_{j},
$$

o qual é aberto em $X$. Mostremos que $x \in U$. De fato, para todo $j=0,1, \ldots, n$ :

$$
x=e x=\left(g_{j}{ }^{-1} g_{j}\right) x=g_{j}{ }^{-1}\left(g_{j} x\right) \in g_{j}{ }^{-1} U_{j}, \quad \text { então } x \in g_{j}{ }^{-1} U_{j}, \quad \forall j=0,1, \ldots, n .
$$

Portanto, $x \in U$. Assim, $U$ é uma vizinhança aberta de $x$ em $X$ e note que

$$
U \subseteq g_{0}{ }^{-1} U_{0}=U_{0}
$$

Resta provar que

$$
g_{i} U \cap U=\emptyset, \quad \forall i=1, \ldots, n .
$$

De fato, para $i=1, \ldots, n$ temos:

$$
\begin{aligned}
g_{i} U & =\left\{g_{i} u \mid u \in U=\bigcap_{j=0}^{n} g_{j}{ }^{-1} U_{j}\right\} \\
& =\left\{g_{i} u \mid u \in g_{j}{ }^{-1} U_{j}, \forall j=0,1, \cdots, n\right\} \\
& =\left\{g_{i} u \mid u=g_{j}{ }^{-1}\left(v_{j}\right), \forall j=0,1, \cdots, n \text { e } v_{j} \in U_{j}\right\} \\
& =\left\{g_{i}\left(g_{j}{ }^{-1}\left(v_{j}\right)\right) \mid v_{j} \in U_{j}, \forall j=0,1, \cdots, n\right\} \\
& =\left\{\left(g_{i} g_{j}{ }^{-1}\right) v_{j} \mid v_{j} \in U_{j}, \forall j=0,1, \cdots, n\right\} \\
& =\bigcap_{j=0}^{n} g_{i} g_{j}{ }^{-1} U_{j} \subseteq g_{i} g_{i}{ }^{-1} U_{i}=e\left(U_{i}\right)=U_{i} .
\end{aligned}
$$


Então, $g_{i} U \subseteq U_{i}$, logo $g_{i} U \cap U \subseteq U_{i} \cap U_{0}=\emptyset$. Assim,

$$
g_{i} U \cap U=\emptyset, \forall i=1, \ldots, n \text {. }
$$

Definição 1.3.20. ((KAWAKUBO, 1991), Chapter 1, Section 1.1, pg. 5) Sejam $(X, G, \varphi)$ e $(Y, G, \psi)$ dois grupos de transformações sobre o mesmo grupo $G$. Dizemos que uma função $f: X \longrightarrow Y$ é uma $G$-aplicação ou uma aplicação $G$-equivariante se:

$$
f(g x)=g f(x), \quad \forall g \in G \text { e } \forall x \in X
$$

lembrando que a ação de $g x$ é dada por $\varphi$ e a ação $g f(x)$ é dada por $\psi$.

Observação 1.3.21. No caso de grupos de transformações topológicos, na Definição 1.3.20 devemos considerar $f: X \rightarrow Y$ uma função contínua.

Definição 1.3.22. ((KAWAKUBO, 1991), Chapter 1, Section 1.1, pg. 5) Uma G-aplicação $f: X \longrightarrow Y$ a qual é também uma bijeção é chamada um $G$-isomorfismo. Quando existe um $G$-isomorfismo $f: X \longrightarrow Y$, dizemos que $X$ e $Y$ são $G$-isomorfos e denotamos $G$-isomorfismos por $X \cong Y$.

Observação 1.3.23. No caso de grupos de transformações topológicos, na Definição 1.3.22, devemos considerar $f: X \rightarrow Y$ um homeomorfismo.

Observação 1.3.24. Um subconjunto $Y$ de um $G$-conjunto $X$ é chamado $G$-invariante se $g Y \subseteq Y$, para qualquer $g \in G$.

Proposição 1.3.25. ((KAWAKUBO, 1991), Chapter 1, Section 1.1, Lemma 1.4, pg. 6) Um subconjunto $G$-invariante $Y$ de um $G$-conjunto $X$ satisfaz $g Y=Y$, para qualquer $g \in G$ e, portanto, a $G$-ação sobre $X$ induz uma $G$-ação sobre $Y$.

Proposição 1.3.26. ((KAWAKUBO, 1991), Chapter 1, Section 1.1, Theorem 1.5, pg. 6) Seja $X$ um $G$-conjunto. Para qualquer $x \in X$, a órbita $G(x)$ é um subconjunto $G$-invariante de $X$ e

$$
G(x) \cong G / G_{x}
$$

onde $G / G_{x}$ é o $G$-conjunto do Exemplo 1.3.5, com $G$-ação $g^{\prime}\left(g G_{x}\right)=\left(g^{\prime} g\right) G_{x}$.

Observação 1.3.27. Se $H$ é um subgrupo de $G$ e se $f: X \longrightarrow Y$ é uma aplicação $G$-equivariante, então $f$ é $H$-equivariante.

Observação 1.3.28. Uma aplicação $G$-equivariante $f: X \longrightarrow Y$ induz uma aplicação entre os espaços de órbitas $\bar{f}: X / G \longrightarrow Y / G$, definida por $\bar{f}(G x)=G f(x), \forall x \in X$. Se $q_{X}: X \rightarrow X / G$ e $q_{Y}: X \rightarrow Y / G$ são as projeções naturais, então $\bar{f}$ é a única aplicação que satisfaz:

$$
\bar{f} \circ q_{X}=q_{Y} \circ f
$$




\subsection{Variedades}

A seguir, apresentamos o conceito de variedades topológicas e algumas de suas importantes propriedades.

Definição 1.4.1. ((LEE, 2010), pg. 38) Seja $n \geq 0$ um inteiro. Um espaço topológico $M$ é localmente Euclideano de dimensão $n$ se para todo ponto $x \in M$, existe um aberto $U \subset M$ que é homeomorfo a um subconjunto aberto do espaço Euclideano $\mathbb{R}^{n}$.

O lema a seguir mostra que, na Definição 1.4.1, podemos substituir conjuntos abertos por bolas abertas ou pelo próprio espaço Euclideano $\mathbb{R}^{n}$.

Lema 1.4.2. ((LEE, 2010), Lemma 2.52, pg. 38) Um espaço topológico $M$ é localmente Euclideano de dimensão $n$ se uma das seguintes condições vale:

(a) Para todo ponto $x \in M$, existe um aberto $U \subset M$ homeomorfo a uma bola aberta em $\mathbb{R}^{n}$.

(b) Para todo ponto $x \in M$, existe um aberto $U \subset M$ homeomorfo a $\mathbb{R}^{n}$.

Definição 1.4.3. ((LEE, 2010), pg. 39) Uma n-dimensional variedade topológica é um espaço topológico Hausdorff $M$, segundo enumerável (ou seja, possui base enumerável) que é localmente Euclideano de dimensão $n$.

Exemplo 1.4.4. ((LEE, 2010), Exercise 2.54, pg. 39) Um espaço topológico $M$ é uma 0-variedade topológica se, e somente se, $M$ for discreto enumerável.

Proposição 1.4.5. ((LEE, 2010), Proposition 4.23, pg. 93) Toda variedade topológica é localmente conexa e localmente conexa por caminhos ${ }^{6}$.

Proposição 1.4.7. ((LEE, 2010), Proposition 4.64, pg. 105) Toda variedade topológica é localmente compacta.

Teorema 1.4.8. ((LEE, 2010), Theorem 4.77, pg. 110) Todo espaço segundo enumerável e localmente compacto Hausdorff é paracompacto. Em particular, toda variedade topológica é paracompacta.

Observação 1.4.9. Como todo espaço paracompacto Hausdorff é normal ((LEE, 2010), Theorem 4.81, pg. 112), segue que toda variedade topológica é um espaço normal, logo completamente regular (pelo Lema de Urysohn) e regular.

Definição 1.4.6. ((HATCHER, 2002), Chapter 1, Section 1.3, pg. 62) Um espaço topológico $X$ é localmente conexo por caminhos se, para cada ponto $x \in X$ e para cada vizinhança aberta $U$ de $x$, existe uma vizinhança aberta $V \subseteq U$ de $x$ que é conexa por caminhos. A definição de conexidade local é análoga. 
Proposição 1.4.10. ((LEE, 2012), Proposition 1.11, pg. 8) Seja $M$ uma variedade topológica. Então, $M$ é conexa se, e somente se, $M$ for conexa por caminhos.

Teorema 1.4.11. Seja $R$ um anel comutativo com identidade.

(a) Se $M$ é uma $n$-variedade compacta e conexa, então $H_{i}(M ; R)=0$, para todo $i>n$ ((HATCHER, 2002), Theorem 3.26(c), pg. 236).

(b) Se $M$ é uma $n$-variedade conexa e não compacta, então $H_{i}(M ; R)=0$, para todo $i \geq n$ ((HATCHER, 2002), Proposition 3.29, pg. 239).

Observação 1.4.12. Seja $R$ um anel comutativo com identidade. Se $M$ é uma $n$-variedade topológica conexa, então seus grupos de homologia singular, $H_{i+1}(M ; R)=0$ para $i \geq n$ (vide Teorema 1.4.11).

Exemplo 1.4.13. Existem exemplos importantes de espaços topológicos que não admitem estrutura de variedade topológica, como segue.

1. Seja $X$ um espaço topológico que não homeomorfo à $n$-esfera $\mathbb{S}^{n}$. Então,

$$
\sum X=(X \times I) /(X \times\{1\} \cup X \times\{0\})
$$

a suspensão não reduzida de $X$, é um espaço topológico que não é uma $(n+1)$-variedade topológica. De fato, dada qualquer vizinhança aberta $U$ de $X \times 0$ em $\sum X$, temos que $U$ é homeomorfa a $C X=(X \times I) /(X \times\{0\})$, o cone de $X$. Porém, $C X$ não é homeomorfo a um disco, uma vez que $\partial(C X)=X$ e $X$ não é homeomorfo à esfera $S^{n}$ (vide Figura 2).

2. Um grafo é um 1-dimensional complexo CW. Todo grafo com pelo menos um vértice elementar (vide Figura 2) é um espaço topológico (com a topologia relativa de $\mathbb{R}^{2}$ ), mas não é uma variedade topológica, pois não é localmente Euclideano (vide (HATCHER, 2002), Example 0.1, pg. 6).

3. A reta de Alexandroff (long line) $L$ é um espaço topológico Hausdorff conexo, localmente homeomorfo à reta real $\mathbb{R}$, mas não possui base enumerável. Assim, $L$ não é uma variedade topológica (vide (SHASTRI, 2011), Example 5.1.1(4), pg. 122).

Definição 1.4.14. ((LEE, 2012), pg. 4) Seja $M$ uma $n$-variedade topológica. Uma carta coordenada (ou apenas uma carta) sobre $M$ é uma par $(U, \varphi)$, onde $U$ é um subconjunto aberto de $M$ e $\varphi: U \rightarrow \hat{U}$ é um homeomorfismo de $U$ em um subconjunto aberto $\hat{U}=\varphi(U) \subset \mathbb{R}^{n}$. Por definição de uma variedade topológica, cada ponto $p \in M$ está contido no domínio de alguma carta $(U, \varphi)$. Se $\varphi(p)=0$, dizemos que a carta está centrada em $p$. Dada uma carta $(U, \varphi)$, chamamos o conjunto $U$ uma vizinhança coordenada de cada 
Figura 2 - Espaços que não são variedades
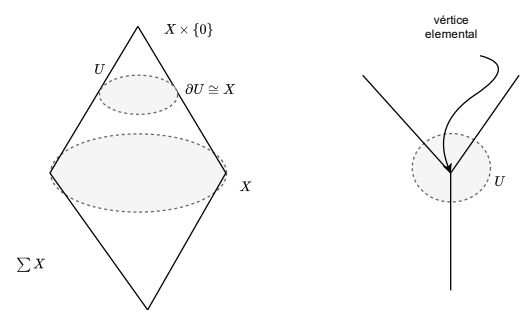

GRAFO

Fonte: Elaborada pelo autor.

um de seus pontos. Se, além disso, $\varphi(U)$ é uma bola aberta em $\mathbb{R}^{n}$, então $U$ é chamada uma bola coordenada. A aplicação $\varphi$ é chamada aplicação coordenada (local) e as funções componentes $\left(x_{1}, \ldots, x_{n}\right)$ de $\varphi$, definidas por $\varphi(p)=\left(x_{1}(p), \ldots, x_{n}(p)\right)$, são chamadas coordenadas locais sobre $U$. Escrevemos, $(U, \varphi)$ é uma carta contendo $p$, para significar que $(U, \varphi)$ é uma carta cujo domínio $U$ contém $p$.

Definição 1.4.15. ((LEE, 2012), pg. 11) Se $U$ e $V$ são conjuntos abertos dos espaços Euclideanos $\mathbb{R}^{n}$ e $\mathbb{R}^{m}$, respectivamente, uma função $F: U \rightarrow V$ é chamada suave (ou de classe $C^{\infty}$ ) se cada uma de suas funções componentes possui derivadas parciais contínuas de todas as ordens. Se, além disso, $F$ for uma bijeção e possui inversa suave, dizemos que $F$ é um difeomorfismo. Um difeomorfismo é, em particular, um homeomorfismo.

Definição 1.4.16. ((LEE, 2010), pg. 12) Seja $M$ uma $n$-variedade topológica. Se $(U, \varphi)$ e $(V, \psi)$ são duas cartas tais que $U \cap V \neq \emptyset$, a composição

$$
\psi \circ \varphi^{-1}: \varphi(U \cap V) \rightarrow \psi(U \cap V)
$$

é chamada função transição de $\varphi$ para $\psi$. Ela é uma composição de homeomorfismos e, portanto, é um homeomorfismo. Duas cartas $(U, \varphi)$ e $(V, \psi)$ são chamadas suavemente compatíveis se $U \cap V=\emptyset$ ou se a função transição $\psi \circ \varphi^{-1}$ é um difeomorfismo. Um atlas para $M$ é uma coleção de cartas cujos domínios cobrem $M$. Um atlas $\mathcal{A}$ é chamado um atlas suave se quaisquer duas cartas em $\mathcal{A}$ são suavemente compatíveis entre si. Um atlas suave $\mathcal{A}$ sobre $M$ é maximal se ele não estiver contido propriamente em qualquer atlas suave "maior". Isso significa que qualquer carta que seja suavemente compatível com cada carta em $\mathcal{A}$, já está em $\mathcal{A}$.

Definição 1.4.17. Se $M$ é uma variedade topológica, uma estrutura suave sobre $M$ é uma atlas maximal suave. Uma variedade suave é um $\operatorname{par}(M, \mathcal{A})$, no qual $M$ é uma variedade topológica e $\mathcal{A}$ é uma estrutura suave sobre $M$.

Exemplo 1.4.18. Para cada inteiro não negativo $n$, são exemplos clássicos de variedades:

(a) O $n$-espaço Euclideano $\mathbb{R}^{n}$ é uma $n$-dimensional variedade topológica (respectivamente suave), ((LEE, 2012), Example 1.22, pg. 17). 
(b) A $n$-esfera $\mathbb{S}^{n}$ é uma $n$-dimensional variedade topológica (respectivamente suave), ((LEE, 2012), Example 1.4, pg.5, Example 1.31, pg. 20).

(c) O n-espaço projetivo real $\mathbb{R P}^{n}$; o conjunto de todos os 1-dimensionais sub-espaços lineares em $\mathbb{R}^{n+1}$ munido da topologia quociente co-induzida pela projeção natural $\pi: \mathbb{R}^{n=1}-\{0\} \rightarrow \mathbb{R P}^{n}$, a qual leva cada ponto $x \in \mathbb{R}^{n+1}-\{0\}$ no sub-espaço linear gerado por $x$; é uma $n$-dimensional variedade topológica (respectivamente suave), ((LEE, 2012), Example 1.5, pg. 6, Example 1.33, pg. 21). Quando $n=2, \mathbb{R P}^{2}$ é chamado o plano projetivo real.

(d) O $n$-espaço projetivo complexo $\mathbb{C P}^{n}$; o conjunto de todos os 1 -dimensionais subespaços lineares complexos em $\mathbb{C}^{n+1}$, munido da topologia quociente co-induzida pela projeção natural $\pi: \mathbb{C}^{n+1}-\{0\} \rightarrow \mathbb{C P}^{n}$; é uma $2 n$-dimensional variedade topológica (respectivamente suave), ((LEE, 2012), Problem 1-9, pg. 31)

(e) O produto finito $M_{1} \times \cdots \times M_{k}$ de variedades topológicas (respectivamente suaves) de dimensões $n_{1}, \ldots, n_{k}$, respectivamente, é uma $\left(n_{1}+\cdots+n_{k}\right)$-variedade topológica (respectivamente suave), ((LEE, 2012), Example 1.8, pg. 7, Example 1.34, pg. 21). Um exemplo importante de uma variedade produto é o produto $T^{n}=\mathbb{S}^{1} \times \cdots \times \mathbb{S}^{1}$ de $n$ cópias da esfera $\mathbb{S}^{1}$, o qual é uma $n$-variedade topológica (respectivamente suave), chamada o $n$-toro. Em particular, o 2-toro $T^{2}=\mathbb{S}^{1} \times \mathbb{S}^{1}$ é uma 2 -variedade topológica (respectivamente suave), chamada simplesmente o toro.

(f) Todo subconjunto aberto de uma $n$-variedade topológica (respectivamente suave) é uma $n$-variedade topológica (respectivamente suave), chamada subvariedade aberta, ((LEE, 2010), Proposition 2.53, pg. 39 e (LEE, 2012), Example 1.26, pg. 19).

(g) Seja $q: E \rightarrow X$ uma aplicação de recobrimento. Se $E$ for uma $n$-variedade topológica e se $X$ for Hausdorff, então $X$ será uma $n$-variedade topológica ((LEE, 2010), Problem 11-1(c), pg. 302).

Teorema 1.4.19. (Classificação de 1-variedades, (LEE, 2010), Theorem 5.27, pg. 145 e (SHASTRI, 2011), Theorem 5.4.1, pg. 133) Toda 1-variedade topológica (respectivamente suave) não vazia e conexa $M$ é homeomorfa (respectivamente difeomorfa) à 1-esfera $\mathbb{S}^{1}$ (caso $M$ seja compacta); e a $\mathbb{R}$ (caso $M$ não seja compacta).

Teorema 1.4.20. (Classificação de 2-variedades, (LEE, 2010), Theorem 6.15, pg. 174 e (SHASTRI, 2011), Theorem 8.5.1, pg. 236) Toda 2-variedade topológica (respectivamente, suave) não vazia, compacta e conexa é homeomorfa (respectivamente, difeomorfa) a um dos seguintes espaços:

(a) A esfera $\mathbb{S}^{n}$

(b) A uma soma conexa de uma ou mais cópias do toro $T^{2}$. 
(c) A uma soma conexa de uma ou mais cópias do plano projetivo real $\mathbb{R} \mathbb{P}^{2}$.

Observação 1.4.21. Toda 2-variedade topológica compacta e conexa possui uma única estrutura suave (a menos de difeomorfismo) ((SHASTRI, 2011), Remark 8.5.3-(4), pg. 242). O mesmo ocorre no caso de 3-variedades topológicas compactas e conexas (vide (MOISE, 2013)).

\subsection{Complexos CW}

Nesta seção, apresentamos o conceito de complexos $C W$ e suas propriedades.

Definição 1.5.1. ((MUNKRES, 1984), Section 38, pg. 214) Um espaço topológico $X$ é chamado uma célula fechada de dimensão $m$ se $X$ é homeomorfo ao $m$-disco unitário

$$
\mathbb{D}^{m}:=\left\{\left(x_{1}, \ldots, x_{m}\right) \in \mathbb{R}^{m}: \sum_{i=1}^{m} x_{i}^{2} \leq 1\right\} .
$$

Exemplo 1.5.2. O $m$-cubo $I^{m}$ e o $m$-simplexo padrão

$$
\Delta^{m}=\left\{\left(x_{0}, x_{1}, \ldots, x_{n}\right) \in \mathbb{R}^{m+1}: \quad x_{i} \geq 0 \text { para } 0 \leq i \leq n e \sum_{i=0}^{m} x_{i}=1\right\}
$$

são $m$-células fechadas.

Definição 1.5.3. ((MUNKRES, 1984), Section 38, pg. 214) Um espaço topológico X é chamado uma célula aberta de dimensão $m$ se $X$ é homeomorfo à $m$-bola aberta

$$
\mathbb{B}^{m}:=\left\{\left(x_{1}, \ldots, x_{m}\right) \in \mathbb{R}^{m}: \sum_{i=1}^{m} x_{i}^{2}<1\right\} .
$$

Usaremos a notação $e_{\alpha}$ para uma $m$-célula aberta.

Exemplo 1.5.4. O interior do $m$-cubo $I^{m}$, int $\left(I^{m}\right)$ e o interior do $m$-simplexo padrão $\operatorname{int}\left(\Delta^{m}\right)$ são $m$-células abertas.

Definição 1.5.5. ((MUNKRES, 1984), Section 38, pg. 214) Um complexo celular ou $C W$ complexo é um espaço topológico $X$ e uma coleção de células abertas disjuntas $e_{\alpha}$ cuja união é $X$, satisfazendo as seguintes propriedades:

1. $X$ é Hausdorff

2. para cada $m$-célula aberta $e_{\alpha}$ da coleção, existe $f_{\alpha}: \mathbb{D}^{m} \longrightarrow X$ contínua, chamada aplicação característica, que $f$ leva o $\operatorname{int}\left(\mathbb{D}^{m}\right)=\mathbb{B}^{m}$ homeomorficamente sobre $e_{\alpha} \mathrm{e}$ leva $\partial \mathbb{D}^{m}=\mathbb{S}^{m-1}$ em uma união finita de células abertas da coleção, cada uma de dimensão menor que $m$.

3. um conjunto $A$ é fechado em $X$ se $A \cap \overline{e_{\alpha}}$ é fechado em $\overline{e_{\alpha}}$, para cada $\alpha$.

O $k$-esqueleto do complexo $X$ é o conjunto de todas as $m$-células de $X$, com $0 \leq m \leq k$. 


\subsection{Sequências Espectrais}

Nesta seção, apresentamos umas das ferramentas mais sofisticadas em Topologia Algébrica, usada para se calcular homologia e cohomologia de espaços: as sequências espectrais. Em particular, a sequência espectral de Leray-Serre.

Seja $R$ uma anel comutativo com identidade.

Definição 1.6.1. Um $R$-módulo filtrado é um par $\left(M, \mathcal{F}_{M}\right)$ onde $M$ é um $R$-módulo e $\mathcal{F}_{M}$ é uma filtração para $M$. Uma filtração decrescente $\mathcal{F}_{M}$ é uma sequência de submódulos de $M$ da forma

$$
\cdots \subseteq F_{n} M \subseteq \cdots \subseteq F_{2} M \subseteq F_{1} M \subseteq F_{0} M=M
$$

e uma filtração crescente $\mathcal{F}_{M}$ é da forma

$$
F_{0} M \subseteq F_{1} M \subseteq F_{2} M \subseteq \cdots \subseteq F_{n} M \subseteq \cdots \subseteq M
$$

Definição 1.6.2. ((WEIBEL, 1994), Chapter 5, Section 5.2, Definition 5.2.1, pg. 122) Uma seqüência espectral do tipo homológica (começando com $E^{2}$ ) de $R$-módulos, consiste de:

1. Uma família $\left\{E_{p q}^{r}\right\}$ de $R$-módulos definida para todos os inteiros $p, q$ e $r \geq 2$.

2. $R$-homomorfismos $d_{p, q}^{r}: E_{p, q}^{r} \longrightarrow E_{p-r, q+r-1}^{r}$ que são diferenciais, no sentido que, $d^{r} \circ d^{r}=0$.

3. $R$-isomorfismo entre $E_{p, q}^{r+1}$ e a homologia de $E_{\star, \star}^{r}$ no ponto $E_{p, q}^{r}$, ou seja,

$$
E_{p, q}^{r+1} \cong \operatorname{Ker}\left(d_{p, q}^{r}: E_{p, q}^{r} \longrightarrow E_{p-r, q+r-1}^{r}\right) / i m\left(d_{p+r, q-r+1}^{r}: E_{p+r, q-r+1}^{r} \longrightarrow E_{p, q}^{r}\right)
$$

Observação 1.6.3. ((WEIBEL, 1994), Chapter 5, Section 5.2, pg. 122)

1. A reta de inclinação $-(r-1) / r$ em $E_{\star, \star}^{r}$ determina um complexo de cadeias (vide Figura 3).

2. Denotaremos

$H_{p, q}\left(E_{\star, \star}^{r}, d^{r}\right):=\operatorname{Ker}\left(d_{p, q}^{r}: E_{p, q}^{r} \longrightarrow E_{p-r, q+r-1}^{r}\right) / i m\left(d_{p+r, q-r+1}^{r}: E_{p+r, q-r+1}^{r} \longrightarrow E_{p, q}^{r}\right)$

a homologia de $E_{\star, \star}^{r}$ no ponto $E_{p, q}^{r}$. Assim, da condição (3) temos:

$$
E_{p, q}^{r+1} \cong H_{p, q}\left(E_{\star, \star}^{r}, d^{r}\right)
$$

isto é, $E_{p, q}^{r+1}$ é um sub-quociente de $E_{p, q}^{r}$. 
Figura 3 - Diferenciais

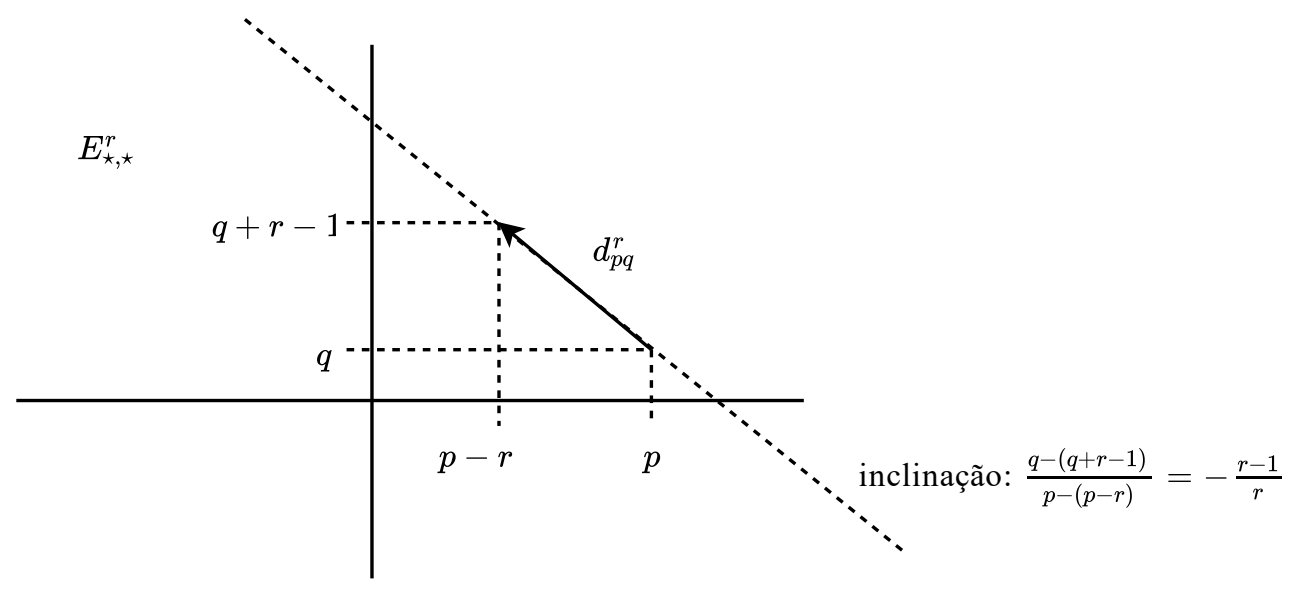

Fonte: Elaborada pelo autor.

3. O grau total do termo $E_{p, q}^{r}$ é $n=p+q$, assim os termos de grau total $n$ pertencem a uma reta de inclinação -1 e cada diferencial $d_{p, q}^{r}$ diminui o grau total por um.

Observação 1.6.4. ((WEIBEL, 1994), Chapter 5, Section 5.2, pg. 122) Existe uma categoria de sequências espectrais do tipo homológica: um morfismo $f: E^{\prime} \longrightarrow E$ é uma família de $R$-homomorfismos $f_{p, q}^{r}: E_{p, q}^{\prime r} \longrightarrow E_{p, q}^{r} \operatorname{com} d^{r} \circ f^{r}=f^{r} \circ d^{r}$ tal que cada $f_{p, q}^{r+1}$ é a aplicação induzida por $f_{p, q}^{r}$ em homologia.

Definição 1.6.5. ((WEIBEL, 1994), Chapter 5, Section 5.2, Example 5.2.2, pg. 122) Dizemos que uma sequência espectral é do primeiro quadrante se $E_{p, q}^{2}=0, \forall p, q<0$ (o que implica que $E_{p, q}^{r}=0, \forall p, q<0, \forall r \geq 2$ ). Se fixamos $p$ e $q$, então $E_{p, q}^{r}=E_{p, q}^{r+1}$ para $r>\max \{p, q+1\}$, pois o diferencial $d^{r}$ que chega em $E_{p, q}^{r}$ vem do quarto quadrante, enquanto o diferencial que sai de $E_{p, q}^{r}$ chega no segundo quadrante. Escrevemos $E_{p, q}^{\infty}$ para este valor estável de $E_{p, q}^{r}$.

Definição 1.6.6. ((WEIBEL, 1994), Chapter 5, Section 5.2, pg. 123) Uma sequência espectral em homologia é chamada limitada, se para cada $n$, existe somente uma quantidade finita de termos não nulos de grau total $n$ em $E_{\star, \star}^{2}$. Isto implica que, para cada $p$ e $q$ existe um $r_{0}$ tal que $E_{p, q}^{r}=E_{p, q}^{r+1}, \forall r \geq r_{0}$. Escrevemos $E_{p, q}^{\infty}$ para este valor estável de $E_{p, q}^{r}$. Note que toda sequência do primeiro quadrante é limitada.

Definição 1.6.7. Dizemos que uma sequência espectral limitada converge para $H_{\star}$, se existe uma família de $R$-módulos $H_{n}$, cada um deles tendo uma filtração finita crescente de longitude $t-s$

$$
0=F_{s} H_{n} \subseteq \cdots \subseteq F_{p-1} H_{n} \subseteq F_{p} H_{n} \subseteq F_{p+1} H_{n} \subseteq \cdots \subseteq F_{t} H_{n}=H_{n}
$$


e isomorfismos $E_{p q}^{\infty} \cong F_{p} H_{p+q} / F_{p-1} H_{p+q}$. A forma tradicional simbólica de descrever tal convergência limitada é:

$$
E_{p, q}^{2} \Rightarrow H_{p+q}
$$

Observação 1.6.8. ((WEIBEL, 1994), Chapter 5, Section 5.2, Example 5.2.6, pg. 124) Se uma sequência espectral (de homologia) do primeiro quadrante converge para $H_{\star}$, então cada $H_{n}$ tem uma filtração finita de longitude $n+1$ :

$$
0=F_{-1} H_{n} \subseteq F_{0} H_{n} \subseteq \cdots \subseteq F_{n-1} H_{n} \subseteq F_{n} H_{n}=H_{n}
$$

Neste caso, o quociente $F_{0} H_{n} / F_{-1} H_{n}=F_{0} H_{n}=E_{0, n}^{\infty}$ de $H_{n}$ fica sobre o eixo $O y$ e o quociente $H_{n} / F_{n-1} H_{n}=E_{n, 0}^{\infty}$ fica sobre o eixo $O x$.

\subsubsection{Sequência espectral de Leray-Serre}

Enunciaremos a seguir um importante teorema para o cálculo de certos grupos de homologia, mais precisamente, grupos de homologia do espaço total de uma fibração.

Teorema 1.6.9 (Sequência espectral de Leray-Serre para Homologia). ((MCCLEARY, 2001), Theorem 5.1, pg. 134) Seja $G$ um grupo abeliano. Suponhamos que $F \stackrel{i}{\longrightarrow} E \stackrel{\pi}{\longrightarrow} B$ seja uma fibração, tal que $B$ e $F$ são conexos por caminhos. Então, existe uma sequência espectral de homologia do primeiro quadrante, $\left\{E_{\star, \star}^{r}, d^{r}\right\}$, convergindo para $H_{\star}(E ; G)$ com

$$
E_{p, q}^{2} \cong H_{p}\left(B, \mathcal{H}_{q}(F ; G)\right)
$$

a homologia do espaço base $B$ com coeficientes locais na homologia da fibra de $\pi$.

Observação 1.6.10. O Teorema 1.6.9 possui uma versão mais simples, onde uma condição é exigida para que o sistema de coeficientes locais seja trivial. Tal versão será usada na Seção 6.1 no cálculo da homologia de alguns espaços de configurações.

Proposição 1.6.11. ((MCCLEARY, 2001), Theorem 5.4, pg. 139) Seja $G$ um grupo abeliano. Dada uma fibração $F \stackrel{i}{\longrightarrow} E \stackrel{\pi}{\longrightarrow} B$, tal que $B$ e $F$ são conexos por caminhos e o sistema de coeficientes locais sobre $B$ determinado pela fibra é simples, equivalentemente, a ação de $\pi_{1}(B)$ sobre $H_{\star}(F ; G)$ é trivial (por exemplo, se $B$ for simplesmente conexo). Então, existe uma sequência espectral de homologia do primeiro quadrante, $\left\{E_{\star, \star}^{r}, d^{r}\right\}$, convergindo para $H_{\star}(E ; G)$ com

$$
E_{p, q}^{2} \cong H_{p}\left(B, H_{q}(F ; G)\right)
$$

Observação 1.6.12. Usualmente para calcular o $E_{p, q}^{2}$ termo na Proposição 1.6.11, fazemos uso do Teorema dos Coeficientes Universais para Homologia. 
Teorema 1.6.13 (Teorema dos Coeficientes Universais para Homologia). ((DAVIS; KIRK, 2001), pg. 44) Seja $R$ um domínio de ideais principais. Suponhamos que $M$ seja um módulo sobre $R$ e que $\left(C_{\star}, \partial\right)$ seja um complexo de cadeias livre sobre $R$ (ou seja, cada $C_{p}$ é um $R$-módulo livre). Então, a sequência

$$
0 \longrightarrow H_{p}\left(C_{\star}\right) \otimes_{R} M \longrightarrow H_{p}\left(C_{\star} ; M\right) \longrightarrow \operatorname{Tor}^{R}\left(H_{p-1}\left(C_{\star}\right) ; M\right) \longrightarrow 0
$$

é exata e cinde.

Observação 1.6.14. Nas mesmas condições do Teorema 1.6.13, note que se $H_{p-1}\left(C_{\star}\right)$ ou se $M$ forem $R$-livres, então

$$
H_{p}\left(C_{\star} ; M\right) \cong H_{p}\left(C_{\star}\right) \otimes_{R} M
$$

Assim, por exemplo, se $R=\mathbb{Z}$ na Proposição 1.6.11, o $E_{p, q}^{2}$ termo da sequência espectral de Leray-Serre é da forma:

$$
E_{p, q}^{2} \cong\left(H_{p}(B) \otimes H_{q}(F)\right) \oplus \operatorname{Tor}^{\mathbb{Z}}\left(H_{p-1}(B) ; H_{q}(F)\right)
$$

Recordemos as propriedades básicas do produto tensorial e do funtor Tor.

Proposição 1.6.15. ((DAVIS; KIRK, 2001), pg. 9) Para $A, B, C,\left\{A_{\alpha}\right\} R$-módulos:

1. $A \otimes_{R} B \cong B \otimes_{R} A$.

2. $R \otimes_{R} B \cong B$.

3. $\left(A \otimes_{R} B\right) \otimes_{R} C \cong A \otimes_{R}\left(B \otimes_{R} C\right)$.

4. $\left(\oplus_{\alpha} A_{\alpha}\right) \otimes_{R} B \cong \oplus_{\alpha}\left(A_{\alpha} \otimes_{R} B\right)$.

5. Dados $R$-homomorfismos $f: A \longrightarrow C$ e $g: B \longrightarrow D$, existe um $R$-homomorfismo $f \otimes_{R} g: A \otimes_{R} B \longrightarrow C \otimes_{R} D$ tal que $a \otimes b \mapsto f(a) \otimes g(b)$.

Proposição 1.6.16. 1. $\operatorname{Tor}^{R}(M, N) \cong \operatorname{Tor}^{R}(N, M)$.

2. $\operatorname{Tor}_{0}^{R}(M, N)=M \otimes_{R} N$.

3. $\operatorname{Tor}_{n}^{R}(M, N)=0, \forall n \geq 1$, se $M$ ou $N$ são $R$-módulos livres. 


\subsection{Espaços classificantes}

O objetivo desta seção é mostrar que todo espaço $C W$ do tipo $K(G, 1)$, com $G$ um grupo discreto e enumerável, é um espaço classificante para $G$.

Definição 1.7.1. Sejam $G$ um grupo e $X$ um espaço topológico conexo por caminhos satisfazendo as seguintes condições:

$$
\pi_{i}(X)=0, \quad \forall i \neq 1, \quad \text { e } \pi_{1}(X) \cong G
$$

Então, $X$ é chamado espaço de Eilenberg-MacLane ou simplesmente um $K(G, 1)$-espaço (vide (HATCHER, 2002), Chapter 4, Section 4.2, pg. 365).

Observação 1.7.2. Mais geralmente, no Capítulo 2 apresentaremos a definição de espaços do tipo $K(G, n)^{7}$, para todo $n \geq 1$ (vide Definição 3.1.34).

Observação 1.7.3. Em toda esta seção, estaremos considerando a definição de um fibrado $\beta=(B, p, X, F, G)$ segundo (STEENROD, 1951), Part I, Section 2, Definições 2.3 e 2.4, pg. 7, 8. De acordo com a nomenclatura adotada por Steenrod, $B$ e $X$ são espaços topológicos, chamados, respectivamente, espaço fibrado e espaço base, $p: B \rightarrow X$ é chamada a projeção e $F$ é chamada a fibra. O espaço $F_{x}=p^{-1}(x)$ é chamado a fibra sobre o ponto $x \in X .(F, G, \varphi)$ é um grupo de transformações topológico, no qual a ação à esquerda $\varphi: G \times F \longrightarrow F$ de $G$ sobre a fibra $F$ é efetiva. $G$ é chamado o grupo do fibrado.

Definição 1.7.4. ((STEENROD, 1951), Part I, Section 8, pg. 35) Um fibrado $\beta=(B, p, X, F, G)$ é chamado um fibrado principal se a fibra $F$ é o grupo $G$.

Definição 1.7.5. ((HATCHER, 2002), Chapter 4, Section 4.1, pg. 346) Um espaço com ponto base $\left(X, x_{0}\right)$ é chamado $n$-conexo, se $\pi_{i}\left(X, x_{0}\right)$ é trivial, para todo $0 \leq i \leq n$. 0 -conexo equivale que o espaço $\left(X, x_{0}\right)$ é conexo por caminhos e 1 -conexo equivale que o espaço $\left(X, x_{0}\right)$ é simplesmente conexo.

Observação 1.7.6. Da Definição 1.7.5, concluímos que se o espaço $\left(X, x_{0}\right)$ for $n$-conexo, então $\pi_{0}\left(X, x_{0}\right)$ é trivial, logo $X$ é conexo por caminhos. Assim, a escolha do ponto base $x_{0}$ não é significativa. Portanto, a condição de um espaço ser $n$-conexo pode ser expressa sem se fazer menção ao ponto base.

Definição 1.7.7. ((STEENROD, 1951), Theorem 19.4, pg. 102) Um fibrado n-universal é um fibrado principal tal que o espaço fibrado é $(n-1)$-conexo. Um fibrado $\infty$-universal ou, simplesmente, um fibrado universal é um fibrado principal que é $n$-universal, para todo $n$, ou seja, quando o espaço fibrado é fracamente contrátil, isto é, o espaço fibrado é conexo por caminhos e seus grupos de homotopia são triviais.

7 Por conveniência, no Capítulo 2 usaremos a notação $K(\pi, n)$ para os espaços de Eilenberg-MacLane. 
Definição 1.7.8. ((MILNOR, 1956), Section 1, pg. 430) Sejam $G$ um grupo topológico e $X$ um espaço topológico. Dizemos que $X$ é um espaço classificante para $G$ se $X$ é o espaço base de algum fibrado universal com grupo $G$.

Observação 1.7.9. Em (HATCHER, 2002), Chapter 1, Section 1.3, Example 1B.7, pg. 89, o autor mostra que para um grupo discreto $G$, existe um complexo $C W$ conexo por caminhos, que é um $K(G, 1)$-espaço. Especificamente, ele constrói um recobrimento universal $^{8} p: E G \rightarrow E G / G=B G$ do espaço de órbitas $B G=E G / G$ tal que $B G$ é um complexo $C W$ que é um $K(G, 1)$-espaço.

Proposição 1.7.10. Seja $G$ um grupo discreto, Então, existe um complexo $C W$ do tipo $K(G, 1)$ que é o espaço classificante para o grupo $G$.

Demonstração. Segue da Observação 1.7.9, que para um grupo discreto $G$, existe um complexo $C W$ conexo por caminhos $B G$ que é um $K(G, 1)$ espaço, o qual possui um recobrimento universal $E G$

$$
p: E G \rightarrow B G
$$

Pela definição de recobrimento universal, $E G$ é simplesmente conexo, ou seja, $E G$ é conexo por caminhos e tem grupo fundamental trivial. Além disso, como $p: E G \rightarrow B G$ é um recobrimento, os grupos de homotopia superiores de $E G$ satisfazem:

$$
\pi_{i}(E G) \cong \pi_{i}(B G), \forall i \geq 2
$$

Sendo $B G$ um $K(G, 1)$-espaço, temos

$$
\pi_{i}(E G) \cong 0, \forall i \geq 2
$$

Assim, o espaço $E G$ é conexo por caminhos e seus grupos de homotopia são triviais. Além disso, toda aplicação de recobrimento é um fibrado principal. Logo $p: E G \rightarrow B G$ é um fibrado universal. Assim, $B G$ é o espaço base do fibrado universal $p: E G \rightarrow B G$ com grupo $G$, ou seja, $B G$ é um espaço classificante para o grupo $G$.

Definição 1.7.11. ((MLNOR, 1956), Section 5, pg. 435) Um grupo topológico $G$ é chamado um $C W$-grupo enumerável se $G$ é um complexo $C W$ enumerável $^{9}$ tal que a aplicação

$$
G \longrightarrow G, \quad g \mapsto g^{-1}
$$

e a aplicação produto

$$
G \times G \longrightarrow G . \quad(g, h) \mapsto g h
$$

são celulares, ou seja, levam o $k$-esqueleto no $k$-esqueleto.

8 vide (HATCHER, 2002), Chapter 1, Section 1.3, pg. 68

9 Isso significa que $G$ possui uma quantidade enumerável de células. 
Exemplo 1.7.12. Todo grupo discreto enumerável é um 0-dimensional complexo CW enumerável, suas 0-células são os elementos de $G$. Assim, $G$ é seu 0-esqueleto. Portanto, as aplicações

$$
G \longrightarrow G, \quad g \mapsto g^{-1}
$$

$\mathrm{e}$

$$
G \times G \longrightarrow G . \quad(g, h) \mapsto g h
$$

são celulares. Assim, $G$ é um $C W$-grupo enumerável.

Lema 1.7.13. ((MILNOR, 1956), Section 5, Theorem 5.2-(1) e -(2), pg. 435)

(1) Qualquer $C W$-grupo enumerável $G$ tem um complexo CW enumerável $X_{1}$ como espaço classificante.

(2) Um segundo complexo CW $X_{2}$ é também um espaço classificante para $G$ se, e somente se, $X_{2}$ tem o mesmo tipo de homotopia que $X_{1}$.

Corolário 1.7.14. Dado um $C W$-grupo enumerável $G$, seja $X_{1}$ um complexo $C W$ que é um espaço classificante para $G$. Então, um complexo $C W X_{2}$ é também um espaço classificante para $G$ se, e somente se, $X_{2}$ tem o mesmo tipo de homotopia que $X_{1}$.

Demonstração. Seja $X$ um complexo CW enumerável que é um espaço classificante para $G$ (Lema 1.7.13-(1)). Por hipótese geral temos que $X_{1}$ é um complexo $C W$ que é um espaço classificante para $G$, logo pelo Lema 1.7.13-(2)

$$
X \simeq X_{1}
$$

Suponhamos que $X_{2}$ seja um espaço classificante para $G$. Vejamos que $X_{2}$ tem o mesmo tipo de homotopia que $X_{1}$. De fato, novamente pelo Lema 1.7.13-(2),

$$
X_{2} \simeq X
$$

Assim, de (1.7.2) e (1.7.1)

$$
X_{2} \simeq X_{1}
$$

Agora, suponhamos que $X_{2} \simeq X_{1}$. Vejamos que $X_{2}$ é um espaço classificante para $G$. De fato, usando (1.7.1) obtemos que $X_{2} \simeq X$. Agora novamente, usando o Lema 1.7.13-(2) segue que $X_{2}$ é um espaço classificante para $G$.

Lema 1.7.15. ((HATCHER, 2002), Chapter 1, Section 1.B, Theorem 1B.8, pg. 90) O tipo de homotopia de um complexo $C W$ do tipo $K(G, 1)$ é unicamente determinado pelo grupo $G$. 
Proposição 1.7.16. Seja $G$ um grupo discreto enumerável. Então, qualquer complexo $C W$ do tipo $K(G, 1)$ é um espaço classificante para o grupo $G$.

Demonstração. Seja $X$ um complexo $C W$ do tipo $K(G, 1)$. Pela Proposição 1.7.10, existe um complexo $C W B G$ do tipo $K(G, 1)$, que é o espaço classificante para o grupo $G$. Agora, pelo Exemplo 1.7.12 $G$ é um $C W$-grupo enumerável. Por outro lado, usando o Lema 1.7.15 obtemos que $X$ tem o mesmo tipo de homotopia que $B G$. Agora, usando o Corolário 1.7.14 segue que $X$ é um espaço classificante para o grupo $G$. 


\section{ESPAÇOS DE CONFIGURAÇÕES}

\subsection{Espaços de Configurações clássicos}

Oscar Zariski, em 1937, utilizou o conceito de espaços de configurações (ZARISKI, 1937). Posteriormente, Ralph Fox, em 1962, definiu o conceito de tranças geométricas usando os espaços de configurações (FOX; NEUWIRTH, 1962). Um estudo detalhado dos espaços de configurações, ordenados e não ordenados, foi apresentado no trabalho de Fadell e Neuwirth (FADELL; NEUWIRTH, 1962).

Uma das características dos espaços de configurações é a sua relação com a teoria de tranças, introduzida por Emil Artin em 1926, a qual é uma grande linha de pesquisa ativa até o momento. Desde então, os espaços de configurações aparecem em distintas áreas de pesquisa: Geometria, Combinatória, Física, Química, Biologia e, mais recentemente, na Robótica.

Um dos problemas em Topologia Algébrica consiste em calcular a homologia e a cohomologia dos espaços de configurações.

(1988) Löffler e Milgram (MILGRAM; LÖFFLER, 1988) calcularam os $\mathbb{Z} / 2$-grupos de homologia de $S F(X, n)$, para qualquer variedade (fechada) $X$.

(1989) Bödigheimer (BÖDIGHEIMER; COHEN; TAYLOR, 1989) calculou os grupos de homologia de $S F(X, n)$, para qualquer variedade $X$ de dimensão ímpar.

Em ambos casos, tais grupos de homologia dependem apenas da homologia da variedade $X$ e de sua dimensão. Em contraste, os números de Betti racionais de $S F(X, n)$ não são determinados pelos números de Betti racionais de $X$, no caso em que $X$ é uma variedade de dimensão par. Quando se trata dos anéis de cohomologia de $S F(X, n)$, um dos problemas é conhecer se tal anel é um invariante homotópico para variedades fechadas $X$. 
(1991) Bendersky e Gitler (BENDERSKY; GITLER, 1991) observaram que os grupos de cohomologia de $F(X, n)$ e $S F(X, n)$ são invariantes homotópicos para variedades $X$ fechadas orientáveis e para variedades formais racionais $X$, tais como variedades projetivas complexas suaves. Eles calcularem os grupos de cohomologia racional de $F(X, n)$ e $S F(X, n)$, explicitamente, em termos do anel de cohomologia racional da variedade $X$.

(1996) Burt Totaro (TOTARO, 1996) mostrou que se $X$ é uma variedade projetiva complexa suave, então os anéis de cohomologia racionais de $F(X, n)$ e $S F(X, n)$ podem ser calculados a partir do anel de cohomologia racional de $X$.

A seguir, apresentamos o conceito de espaços de configurações.

Definição 2.1.1. ((COHEN, 1995), Section 1, pg. 20) Seja $X$ um espaço topológico. Defina o espaço de configurações ordenado de $k$ pontos distintos em $X$ como o subespaço de $X^{k}$ dado por

$$
F(X, k):=\left\{\left(x_{1}, \ldots, x_{k}\right) \in X^{k}: x_{i} \neq x_{j} \quad \text { se } i \neq j\right\}
$$

Os espaços de configurações ordenados são conhecidos também como espaços de configurações clássicos.

Diretamente da Definição 2.1.1, obtemos as seguintes observações.

Observação 2.1.2. Se o número de elementos de $X$, denotado $|X|$, é estritamente menor que $k$, então $F(X, k)=\emptyset$.

\section{Observação 2.1.3.}

$$
F(X, k)=X^{k}-\bigcup_{i<j} H_{i, j}
$$

onde $H_{i, j}=\left\{\left(x_{1}, \ldots, x_{k}\right) \in X^{k}: x_{i}=x_{j}\right\}$.

Uma propriedade básica dos espaços de configurações é a existência de uma ação livre do grupo simétrico $S_{k}$ sobre $F(X, k)$.

Proposição 2.1.4. ((COHEN, 2010), Definition 2.1, pg. 184) O grupo simétrico sobre $k$-letras, $S_{k}$, age livremente sobre $F(X, k)$ (à esquerda) permutando coordenadas, como segue:

$$
\varphi: S_{k} \times F(X, k) \longrightarrow F(X, k), \varphi\left(\sigma,\left(x_{1}, \ldots, x_{k}\right)\right):=\left(x_{\sigma(1)}, \ldots, x_{\sigma(k)}\right) .
$$


1. Para $i d \in S_{k}$, a permutação identidade, e para $\left(x_{1}, \ldots, x_{k}\right) \in F(X, k)$, temos:

$$
\begin{aligned}
\varphi\left(i d,\left(x_{1}, \ldots, x_{k}\right)\right) & =\left(x_{i d(1)}, \ldots, x_{i d(k)}\right) \\
& =\left(x_{1}, \ldots, x_{k}\right) .
\end{aligned}
$$

2. Para quaisquer $\sigma_{1}, \sigma_{2} \in S_{k}$ e $\left(x_{1}, \ldots, x_{k}\right) \in F(X, k)$ :

$$
\begin{aligned}
\varphi\left(\sigma_{1}, \varphi\left(\sigma_{2},\left(x_{1}, \ldots, x_{k}\right)\right)\right) & =\varphi\left(\sigma_{1},\left(x_{\sigma_{2}(1)}, \ldots, x_{\sigma_{2}(k)}\right)\right) \\
& \left.=\left(x_{\sigma_{1} \sigma_{2}(1)}, \ldots, x_{\sigma_{1} \sigma_{2}(k)}\right)\right) \\
& =\varphi\left(\sigma_{1} \sigma_{2},\left(x_{1}, \ldots, x_{k}\right)\right) .
\end{aligned}
$$

Note que essa ação é livre, pois apenas o elemento identidade fixa qualquer ponto $\left(x_{1}, \ldots, x_{k}\right) \in F(X, k)$. Resta provar que a ação $\varphi$ é continua. Como o grupo $G=S_{k}$ é discreto, pela Observação 1.3.6 é suficiente provar que para cada $\sigma \in S_{k}$, a aplicação

$$
\varphi_{\sigma}: F(X, k) \longrightarrow F(X, k)
$$

é contínua. De fato, note que a aplicação $\varphi_{\sigma}: F(X, k) \longrightarrow F(X, k)$ é a restrição, ao espaço de configurações $F(X, k)$, da aplicação (usando a mesma notação)

$$
\varphi_{\sigma}: X^{k} \longrightarrow X^{k}, \quad\left(x_{1}, \ldots, x_{k}\right) \mapsto\left(x_{\sigma(1)}, \ldots, x_{\sigma(k)}\right) .
$$

Assim, basta provar que esta última aplicação é contínua. Seja $\prod_{i=1}^{k} U_{i}$ um aberto básico para $X^{k}$, então $\varphi_{\sigma}^{-1}\left(\prod_{i=1}^{k} U_{i}\right)=\prod_{i=1}^{k} U_{\sigma^{-1}(i)}$ é aberto em $X^{k}$. Portanto, $\varphi_{\sigma}$ é continua.

Observação 2.1.5. Note que a ação dada na Proposição 2.1.4, pode ser considerada também como uma ação à direita. Por essa razão, dizemos simplesmente que o grupo simétrico $S_{k}$ age sobre o espaço de configurações $F(X, k)$.

Definição 2.1.6. ((COHEN; PAKIANATHAN, ) Definition 2.1, pg. 3; (COHEN, 1995) Section 1, pg. 20) Dado um espaço topológico $X$, podemos considerar o espaço de órbitas

$$
S F(X, k):=B(X, k):=F(X, k) / S_{k},
$$

chamado o espaço de configurações não ordenado.

Observação 2.1.7. Dado um espaço de Hausdorff $X$, como o grupo finito $S_{k}$ age livremente sobre o espaço de Hausdorff $F(X, k)$, temos pela Proposição 1.3 .19 que essa ação é propriamente descontínua. Assim, a aplicação quociente

$$
q: F(X, k) \longrightarrow S F(X, k)
$$

é uma aplicação de recobrimento ((COHEN; PAKIANATHAN, ) Section 2, pg. 3). 
Observação 2.1.8. No caso em que $F(X, k)$ é simplesmente conexo, usando o Corolário B.3.3, temos:

$$
\pi_{1}(S F(X, k)) \cong S_{k}
$$

Observação 2.1.9. Dentre os espaços de configurações mais conhecidos estão os espaços $S F\left(\mathbb{R}^{2}, k\right)$ e $F\left(\mathbb{R}^{2}, k\right)$. Fadell e Neuwirth mostraram que $S F\left(\mathbb{R}^{2}, k\right)$ é um $K(\pi, 1)$-espaço de Eilenberg-MacLane, com grupo fundamental isomorfo ao grupo de tranças de Artin sobre $k$-cordas $B_{k}$ (vide (FADELL; NEUWIRTH, 1962)). Usando o fato que $F\left(\mathbb{R}^{2}, k\right)$ é um recobrimento para $S F\left(\mathbb{R}^{2}, k\right)$ segue que $F\left(\mathbb{R}^{2}, k\right)$ é também um $K(\pi, 1)$-espaço de Eilenberg-MacLane, com grupo fundamental isomorfo ao grupo de tranças puras sobre $k$-cordas $P B_{k}$ (vide (COHEN, 2010), Section 2, Example 2.2, pg. 184). Assim, a cohomologia de $S F\left(\mathbb{R}^{2}, k\right)$ e $F\left(\mathbb{R}^{2}, k\right)$ é justamente a cohomologia do grupo de tranças $B_{k}$ e do grupo de tranças puras $P B_{k}$, respectivamente. Tais cohomologias foram obtidas pelos seguintes autores:

Arnold (ARNOL'D, 1969): calculou a cohomologia de $F\left(\mathbb{R}^{2}, k\right)$.

Fuks (FUKS, 1970) e Cohen (COHEN, 1973) calcularam a cohomologia de $S F\left(\mathbb{R}^{2}, k\right)$.

Exemplo 2.1.10. A seguir, apresentamos alguns exemplos.

(1) $F(X, 1)$ e $S F(X, 1)$ são ambos iguais a $X$ ((COHEN; PAKIANATHAN, ), Remark 2.2 , pg. 3).

(2) $F\left(\mathbb{R}^{n}, 2\right) \simeq \mathbb{S}^{n-1}((\mathrm{COHEN}, 2010)$, Example 2.3, pg. 185).

(3) $F\left(\mathbb{R}^{n}, 2\right) \simeq \mathbb{R}^{n}-\{0\}$.

(4) $F\left(\mathbb{R}^{n}, k\right)$ é homeomorfo a $\mathbb{R}^{n} \times F\left(\mathbb{R}^{n}-\{0\}, k-1\right)((\mathrm{COHEN}, 2010)$, Example 2.3, pg. 185).

(5) Se $G$ é um grupo topológico com elemento identidade $e$, então $F(G, k)$ é homeomorfo a $G \times F(G-\{e\}, k-1), \forall k \geq 2$. ((COHEN, 2010), Example 2.6, pg. 186).

(6) $F\left(\mathbb{S}^{n}, 2\right) \simeq \mathbb{S}^{n}((\mathrm{COHEN}, 2010)$, Example 2.4, pg. 186).

Demonstração. (1) Segue da Definição 2.1.1.

(2) Prova (i) Definimos as funções contínuas:

$$
f: \mathbb{S}^{n-1} \longrightarrow F\left(\mathbb{R}^{n}, 2\right), \quad f(x)=(x, 0) \text { e } g: F\left(\mathbb{R}^{n}, 2\right) \longrightarrow \mathbb{S}^{n-1}, g(x, y)=\frac{x-y}{\|x-y\|} .
$$


Note que $g \circ f=i d_{\mathbb{S}^{n-1}}$. Consideremos a homotopia $H: F\left(\mathbb{R}^{n}, 2\right) \times I \longrightarrow F\left(\mathbb{R}^{n}, 2\right)$ (linear) definida por:

$H((x, y), t)=(1-t)(x, y)+t\left(\frac{x-y}{\|x-y\|}, 0\right)=\left(t\left(\frac{x-y}{\|x-y\|}-x\right)+x,(1-t) y\right)$.

Então, para todo $(x, y) \in F\left(\mathbb{R}^{n}, 2\right)$, temos:

$H((x, y), 0)=(x, y)=I d_{F\left(\mathbb{R}^{n}, 2\right)}(x, y)$ e $H((x, y), 1)=\left(\frac{(x-y)}{\|x-y\|}, 0\right)=(f \circ g)(x, y)$.

Portanto, $f \circ g \stackrel{H}{\simeq} i d_{F\left(\mathbb{R}^{n}, 2\right)}$.

(2) Prova (ii) Alternativamente, podemos considerar as funções contínuas:

$f: \mathbb{S}^{n-1} \longrightarrow F\left(\mathbb{R}^{n}, 2\right), f(x)=(x,-x)$ e $g: F\left(\mathbb{R}^{n}, 2\right) \longrightarrow \mathbb{S}^{n-1}, g(x, y)=\frac{x-y}{\|x-y\|}$.

Neste caso, temos $g \circ f=i d_{\mathbb{S}^{n-1}} \quad$ e $\quad f \circ g \stackrel{H}{\simeq} i d_{F\left(\mathbb{R}^{n}, 2\right)}$, onde

$$
H: F\left(\mathbb{R}^{n}, 2\right) \times I \longrightarrow F\left(\mathbb{R}^{n}, 2\right)
$$

é a homotopia (linear) definida por:

$$
\begin{aligned}
H((x, y), t) & =(1-t)(x, y)+t\left(\frac{x-y}{\|x-y\|},-\frac{x-y}{\|x-y\|}\right) \\
& =\left((1-t) x+\frac{t}{\|x-y\|}(x-y),(1-t) y-\frac{t}{\|x-y\|}(x-y)\right) .
\end{aligned}
$$

(3) Definimos as funções contínuas:

$f: \mathbb{R}^{n}-\{0\} \longrightarrow F\left(\mathbb{R}^{n}, 2\right), f(x)=(x, 0)$ e $g: F\left(\mathbb{R}^{n}, 2\right) \longrightarrow \mathbb{R}^{n}-\{0\}, g(x, y)=x-y$.

Note que $g \circ f=i d_{\mathbb{R}^{n}-\{0\}} \quad$ e $\quad f \circ g \stackrel{H}{\simeq} i d_{F\left(\mathbb{R}^{n}, 2\right)}$, onde

$$
H: F\left(\mathbb{R}^{n}, 2\right) \times I \longrightarrow F\left(\mathbb{R}^{n}, 2\right)
$$

é a homotopia (linear) definida por:

$$
H((x, y), t)=(1-t)(x, y)+t(x-y, 0)=(x-t y,(1-t) y)
$$

(4) Definimos as funções contínuas:

$f: F\left(\mathbb{R}^{n}, k\right) \longrightarrow \mathbb{R}^{n} \times F\left(\mathbb{R}^{n}-\{0\}, k-1\right), f\left(x_{1}, \ldots, x_{k}\right)=\left(x_{1},\left(x_{2}-x_{1}, \ldots, x_{k}-x_{1}\right)\right) e$ $g: \mathbb{R}^{n} \times F\left(\mathbb{R}^{n}-\{0\}, k-1\right) \longrightarrow F\left(\mathbb{R}^{n}, k\right), g\left(x_{1},\left(x_{2}, \ldots, x_{k}\right)\right)=\left(x_{1}, x_{2}+x_{1}, \ldots, x_{k}+x_{1}\right)$.

Note que $f \circ g=i d_{R^{n} \times F\left(\mathbb{R}^{n}-\{0\}, k-1\right)} \quad$ e $\quad g \circ f=i d_{F\left(\mathbb{R}^{n}, k\right)}$. 
(5) Similarmente ao ítem anterior, definimos o homeomorfismo

$$
h: F(G, k) \longrightarrow G \times F(G-\{e\}, k-1), \quad h\left(g_{1}, \ldots, g_{k}\right)=\left(g_{1},\left(g_{2} g_{1}^{-1}, \ldots, g_{k} g_{1}^{-1}\right)\right)
$$

com inversa

$$
f: G \times F(G-\{e\}, k-1) \longrightarrow F(G, k), \quad f\left(g,\left(g_{1} \ldots, g_{k-1}\right)\right)=\left(g, g_{1} g, g_{2} g, \ldots, g_{k-1} g\right)
$$

(6) Definimos as funções contínuas

$$
f: \mathbb{S}^{n} \longrightarrow F\left(\mathbb{S}^{n}, 2\right), \quad f(x)=(x,-x) \text { e } g: F\left(\mathbb{S}^{n}, 2\right) \longrightarrow \mathbb{S}^{n}, \quad g(x, y)=x
$$

Note que $g \circ f=i d_{\mathbb{S}^{n}} \quad$ e $\quad f \circ g \stackrel{H}{\simeq} i d_{F\left(\mathbb{S}^{n}, 2\right)}$, onde

$$
H: F\left(\mathbb{S}^{n}, 2\right) \times I \longrightarrow F\left(\mathbb{S}^{n}, 2\right)
$$

é a homotopia definida por

$$
H((x, y), t)=\left(x, \frac{-t x+(1-t) y}{\|-t x+(1-t) y\|}\right) .
$$

Usando o linguagem de categorias (Definição 1.1.1), podemos pensar nos espaços de configurações como funtores.

Observação 2.1.11. ((COHEN; PAKIANATHAN, ), Remark 2.3, pg. 3) Consideremos $\mathfrak{T o p}_{i}$ a categoria cujos objetos são espaços topológicos $X$, com $X$ não finito, e cujos morfismos são as aplicações contínuas injetoras. Então, para cada $k \geq 1$,

$$
F(-, k): \mathfrak{T o p}_{i} \longrightarrow \mathfrak{T o p}_{i}
$$

é um funtor covariante da categoria $\mathfrak{T o p}_{i}$ na categoria $\mathfrak{T} \mathfrak{p p}_{i}$, o qual associa a cada espaço $X$ o espaço de configurações $F(X, k)$ e a cada função contínua e injetora $f: X \longrightarrow Y$, associa a função contínua e injetora $F(f, k): F(X, k) \longrightarrow F(Y, k)$ definida como segue:

$$
F(f, k)\left(x_{1}, \ldots, x_{k}\right)=\left(f\left(x_{1}\right), \ldots, f\left(x_{k}\right)\right)
$$

Note ainda que $F(f, k)$ é $S_{k}$-equivariante, ou seja,

$$
F(f, k)(\sigma x)=\sigma F(f, k)(x), \forall \sigma \in S_{k}, \quad \forall x \in F(X, k)
$$

Uma aplicação contínua $H: X \times I \longrightarrow Y$ tal que $H_{t}$ é um morfismo na categoria $\mathfrak{T o p}_{i}$, para cada $t \in I$, ou seja, $H_{t}$ é injetora, para cada $t \in I$, é chamada uma isotopia. Tal aplicação induz uma homotopia $H^{*}:=F(H): F(X, k) \times I \longrightarrow F(Y, k)$ definida por:

$$
H^{*}\left(\left(x_{1}, \ldots, x_{k}\right), t\right)=\left(H\left(x_{1}, t\right), \ldots, H\left(x_{k}, t\right)\right)
$$


Observemos que $H^{*}$ também é uma isotopia e, para cada $t \in I, H_{t}^{*}$ é $S_{k}$-equivariante.

Para o caso não ordenado, também temos que $S F(-, k): \mathfrak{T o p}_{i} \longrightarrow \mathfrak{T o p}_{i}$ é um funtor covariante, o qual associa a cada espaço topológico $X$, o espaço de configurações $S F(X, k)$ e, a cada função contínua e injetora $f: X \longrightarrow Y$, associa a função contínua e injetora $S F(f, k): S F(X, k) \longrightarrow S F(Y, k)$ definida como segue:

$$
S F(f, k)[x]=[F(f, k)(x)]=\left[\left(f\left(x_{1}\right), \ldots, f\left(x_{k}\right)\right)\right] .
$$

Note que $S F(f, k)$ é bem definida, pois $F(f, k)$ é $S_{k}$-equivariante. Uma aplicação contínua $H: X \times I \longrightarrow Y$ tal que $H_{t}$ é um morfismo na categoria $\mathfrak{T o p}_{i}$, para cada $t \in I$, induz uma homotopia $H^{* *}:=S F(H): S F(X, k) \times I \longrightarrow S F(Y, k)$ definida por:

$$
H^{* *}([x], t)=[F(H)(x, t)]=\left[H_{t}^{*}(x)\right]
$$

a qual é bem definida, pois $H_{t}^{*}$ é $S_{k}$-equivariante, para cada $t \in I$.

No caso em que $k=1$, tem-se os funtores identidade.

Observação 2.1.12. Um dos problemas interessantes nesta teoria foi saber se os funtores $F(-, k)$ e $S F(-, k)$ são invariantes homotópicos. Mas, Paolo Salvatore em (LONGONI; SALVATORE, 2005), apresenta um contraexemplo de dois espaços equivalentes homotópicos, com espaços de configurações $F(-, k)$ não equivalente homotópicos.

Usando a definição de espaços de configurações, obtemos a seguinte

Proposição 2.1.13. São válidos os seguintes resultados:

1. Se $Y$ é subespaço de $X$, então $F(Y, k)$ é subespaço de $F(X, k), \forall k \geq 1$.

2. Se $X$ é homeomorfo a $Y$, então $F(X, k)$ é homeomorfo a $F(Y, k), \forall k \geq 1$.

Demonstração. 1. Se $Y$ é subespaço de $X$, denotemos por

$$
\tau_{Y^{k}}:=\left\{U \cap F(Y, k): U \text { é aberto em } Y^{k}\right\}
$$

a topologia relativa de $F(Y, k)$ com respeito a $Y^{k}$ e

$$
\tau_{F(X, k)}:=\{U \cap F(Y, k): U \text { é aberto em } F(X, k)\}
$$

a topologia relativa de $F(Y, k)$ com respeito a $F(X, k)$. Vejamos que $\tau_{Y^{k}}=\tau_{F(X, k)}$. De fato, seja $V \in \tau_{Y^{k}}$ então $V=U \cap F(Y, k) \operatorname{com} U$ aberto em $Y^{k}$. Como $Y^{k}$ é subespaço de $X^{k}$, existe $W$ aberto em $X^{k}$ tal que $U=W \cap Y^{k}$. Assim,

$$
V=W \cap Y^{k} \cap F(Y, k)=W \cap F(Y, k)=(W \cap F(X, k)) \cap F(Y, k) .
$$

Como $W \cap F(X, k)$ é aberto em $F(X, k)$, segue que $V \in \tau_{F(X, k)}$. 
Agora, seja $V \in \tau_{F(X, k)}$. Então, $V=U \cap F(Y, k)$, com $U$ aberto em $F(X, k)$. Como $F(X, k)$ é subespaço de $X^{k}$, existe $W$ aberto em $X^{k}$ tal que $U=W \cap F(X, k)$. Assim,

$$
V=W \cap F(X, k) \cap F(Y, k)=W \cap F(Y, k)=\left(W \cap Y^{k}\right) \cap F(Y, k) .
$$

Como $W \cap Y^{k}$ é aberto em $Y^{k}$, segue que $V \in \tau_{Y^{k}}$.

2. Se $X$ é homeomorfo a $Y$, como $F(-, k): \mathfrak{T o p}_{i} \rightarrow \mathfrak{T o p} \mathfrak{p}_{i}$ é um funtor covariante, para cada $k \geq 1$, segue que $F(X, k)$ é homeomorfo a $F(Y, k), \forall k \geq 1$.

Corolário 2.1.14. Sejam $X, Y$ espaços topológicos. Se $F(X, k)$ não for equivalente homotópico a $F(Y, k)$ para algum $k \geq 1$, então $X$ e $Y$ não serão homeomorfos.

Mediante a Proposição 2.1.13, podemos calcular o espaço de configurações para a variedade aberta $\mathbb{S}^{n}-\{\star\}$.

Exemplo 2.1.15. $F\left(\mathbb{S}^{n}-\{\star\}, 2\right) \simeq \mathbb{S}^{n-1}$.

Demonstração. Sabemos que $\mathbb{S}^{n}-\{\star\}$ é homeomorfa ao $\mathbb{R}^{n}$ (via a projeção estereográfica), assim pela Proposição 2.1.13 (2), $F\left(\mathbb{S}^{n}-\{\star\}, 2\right)$ é homeomorfo a $F\left(\mathbb{R}^{n}, 2\right)$ e do Exemplo 2.1.10-(2), temos $F\left(\mathbb{R}^{n}, 2\right) \simeq \mathbb{S}^{n-1}$.

Observação 2.1.16. Note que, se $A \subseteq X$, então $F(A, k)=A^{k} \cap F(X, k), \forall k \geq 1$. Assim, quando $A$ for fechado (respectivamente aberto) em $X$, então $F(A, k)$ será fechado (respectivamente aberto) em $F(X, k)$.

Proposição 2.1.17. Se $X$ é um espaço de Hausdorff, então $F(X, k)$ é aberto em $X^{k}$.

Demonstração. Prova (i) Seja $x=\left(x_{1}, \ldots, x_{k}\right) \in F(X, k)$. Como $X$ é Hausdorff e $x_{i} \neq x_{j}$ sempre que $i \neq j$, então para cada $i, j=1, \ldots, k$ existem $U_{i}, U_{j}$ vizinhanças abertas para $x_{i}, x_{j}$, com $U_{i} \cap U_{j}=\emptyset$, para todo $i \neq j$. Considerando $U=U_{1} \times \cdots \times U_{k}$ aberto em $X^{k}$, temos que $x \in U$. Vejamos que $U \subseteq F(X, k)$. De fato, dado $y=\left(y_{1}, \ldots, y_{k}\right) \in U$, suponhamos que existam $i, j, \operatorname{com} i \neq j$ tais que $y_{i}=y_{j} \in U_{i} \cap U_{j}$. Então, $y_{i} \in U_{i} \cap U_{j}$, o que é uma contradição. Portanto, $y \in F(X, k)$. Assim $U \subseteq F(X, k)$.

Prova (ii) Pela Observação 2.1.3, temos $F(X, k)=X^{k}-\bigcup_{i<j} H_{i, j}$, onde $H_{i, j}=\left\{\left(x_{1}, \ldots, x_{k}\right) \in X^{k}: x_{i}=x_{j}\right\}$. Assim, basta mostrar que

$$
H_{i, j}=\left\{\left(x_{1}, \ldots, x_{k}\right) \in X^{k}: x_{i}=x_{j}\right\}
$$

é fechado $\forall i<j$. De fato, provaremos que

$$
X^{k}-H_{i, j}
$$


é aberto. Seja $x=\left(x_{1}, \ldots, x_{k}\right) \in X^{k}-H_{i, j}$, então $x_{i} \neq x_{j}$, para todo $i \neq j$. Como $X$ é Hausdorff, existem $U_{i}, U_{j}$ abertos de $X$ tais que $x_{i} \in U_{i}, x_{j} \in U_{j}$ e $U_{i} \cap U_{j}=\emptyset$. Consideremos $U=\prod_{s=1}^{k} V_{s}$ aberto de $X^{k}$, onde

$$
V_{s}= \begin{cases}U_{s}, & \text { se } s=i, j \\ X, & \text { caso contrário. }\end{cases}
$$

Como $U_{i} \cap U_{j}=\emptyset$, segue que $U \subseteq X^{k}-H_{i, j}$. Portanto, $X^{k}-H_{i, j}$ é aberto.

Observação 2.1.18. 1. Pela prova $(i)$ da Proposição 2.1.17 temos que se $X$ é um espaço de Hausdorff, então cada ponto $x=\left(x_{1}, \ldots, x_{k}\right) \in F(X, k)$ possui uma vizinhança aberta da forma $\prod_{i=1}^{k} U_{i}$, com $U_{i}$ aberto em $X$ e tal que $U_{i} \cap U_{j}=\emptyset$, para todo $1 \leq i \neq j \leq k$.

2. Similarmente, se $X$ é Hausdorff, então para cada aberto $U$ em $F(X, k)$ e para cada ponto $x \in U$, existe uma vizinhança aberta para $x$ da forma $\prod_{i=1}^{k} U_{i}$, com $U_{i}$ aberto em $X$, e tal que $U_{i} \cap U_{j}=\emptyset$, para todo $1 \leq i \neq j \leq k$, pois, como $F(X, k)$ é aberto em $X^{k}$, então todo aberto em $F(X, k)$ é também um aberto do espaço produto $X^{k}$. Assim, $x$ está no aberto $U$ e como os conjuntos da forma $\prod_{i=1}^{k} U_{i}$, com $U_{i}$ aberto em $X$, formam uma base para o espaço produto $X^{k}$, então existe $\prod_{i=1}^{k} U_{i}$, com $U_{i}$ aberto em $X$, tal que $x \in \prod_{i=1}^{k} U_{i} \subseteq U \subseteq F(X, k)$. A propriedade $U_{i} \cap U_{j}=\emptyset, \forall 1 \leq i \neq j \leq k$ segue do fato que $\prod_{i=1}^{k} U_{i} \subseteq F(X, k)$.

3. Uma base para o espaço de configurações $F(X, k)$, com $X$ Hausdorff, é dada pelos abertos da forma $\prod_{i=1}^{k} U_{i}$, com $U_{i}$ aberto em $X$, e tal que $U_{i} \cap U_{j}=\emptyset, \forall 1 \leq i \neq j \leq k$.

Teorema 2.1.19. ((FADELL; NEUWIRTH, 1962), Section II, pg.111) Se $M$ é uma $n$-variedade topológica (respetivamente, suave), então $F(M, k)$ e $S F(M, k)$ são $(n k)$ variedades topológicas (respectivamente, suaves).

Demonstração. Como $M^{k}$ é Hausdorff, segue da Proposição 2.1.17 que $F(M, k)$ é um conjunto aberto da $(n k)$-variedade topológica (respetivamente suave) $M^{k}$ e do Exemplo 1.4.18-(f), temos que $F(M, k)$ é uma ( $n k)$-variedade topológica (respectivamente suave). Além disso, da Observação 2.1.7, temos a aplicação de recobrimento

$$
q: F(M, k) \longrightarrow S F(M, k)
$$

e como $S F(M, k)$ é Hausdorff (pela Proposição 1.3.16-(a)), temos do Exemplo 1.4.18(g) que $S F(M, k)$ é uma $(n k)$-variedade topológica. Mais ainda, usando ((LEE, 2012), Theorem 21.10, pg. 544), concluímos que $S F(M, k)$ também é uma $(n k)$-variedade suave (lembrando que todo grupo discreto é um grupo de Lie 0-dimensional). 
Observação 2.1.20. Pelo Teorema 2.1.19, no caso em que $M$ é uma variedade suave, podemos usar as ferramentas da Topologia Diferencial para estudar os espaços de configurações $F(M, k)$ e $S F(M, k)$.

O resultado a seguir afirma que, no caso de variedades suaves, os espaços de configurações possuem uma estrutura de complexo $C W$.

Corolário 2.1.21. Se $M$ é uma variedade suave, então os espaços de configurações $F(M, k)$ e $S F(M, k)$ são complexos $C W$.

Demonstração. Pelo Teorema 2.1.19, sabemos que se $M$ é uma variedade suave, então os espaços de configurações $F(M, k)$ e $S F(M, k)$ são variedades suaves. Portanto, de ((DAVIS; KIRK, 2001), pg. 345) concluímos que $F(M, k)$ e $S F(M, k)$ são complexos $C W$.

Uma questão natural que surge com relação aos espaços de configurações é a seguinte:

Problema 1. Dados um espaço topológico $X$ e inteiros não negativos $k$ e $l$, então $F(X, k)$ é homeomorfo a $F(X, l)$ se, e somente se, $k=l$ ?

Observação 2.1.22. Em geral, a questão colocada no Problema 1 não é verdadeira, como mostram os exemplos a seguir:

1. Se $X=\{\star\}$, então para $k=2$ e $l=3$, os espaços de configurações $F(X, 2)$ e $F(X, 3)$ são ambos vazios.

2. Se $X$ é um conjunto finito, denotando por $|X|$ o número de elementos de $X$, temos:

$$
F(X,|X|+1)=\emptyset=F(X,|X|+2),
$$

$\operatorname{com}|X|+1 \neq|X|+2$.

No caso de variedades topológicas, o resultado a seguir fornece uma resposta afirmativa para a questão colocada no Problema 1.

Proposição 2.1.23. Sejam $M$ uma $m$-variedade topológica, com $m \geq 1$, e $k, l$ inteiros não negativos. Então, $F(M, k)$ é homeomorfo a $F(M, l)$ se, e somente se, $k=l$.

Demonstração. Pelo Teorema 2.1.19, sabemos que $F(M, k)$ e $F(M, l)$ são variedades topológicas de dimensões $k m$ e $l m$, respectivamente. Agora, se $F(M, k)$ for homeomorfo a $F(M, l)$, segue do Teorema da Invariância Topológica da Dimensão (vide (LEE, 2012), Theorem 1.2, pg. 3), que $k m=l m$ e, portanto, $k=l$.

No resultado a seguir, usaremos o fato que em uma variedade topológica a conexidade é equivalente à conexidade por caminhos (vide Proposição 1.4.10). 
Proposição 2.1.24. Sejam $M$ uma $n$-variedade topológica e $F \subseteq M$ finito, com $n \geq 2$. Se $M$ for conexa, então $M-F$ será uma $n$-variedade topológica conexa.

Demonstração. $M-F$ é aberto em $M$ e segue do Exemplo 1.4.18-(f) que $M-F$ é uma $n$-variedade topológica. Mostremos que $M-F$ é conexa. O resultado seguirá por indução sobre a cardinalidade $|F|$ de $F$. Suponhamos que $F=\{q\}$ e denotemos por $M^{\prime}=M-\{q\}$. Dados $x, y \in M^{\prime}$, consideremos os caminhos:

$$
\alpha, \beta:[0,1] \longrightarrow M
$$

tais que $\alpha(0)=x, \alpha(1)=q, \beta(0)=q$ e $\beta(1)=y$. Sem perda de generalidade, podemos supor que $q \notin \alpha([0,1))$ e $q \notin \beta((0,1])$. Seja $U$ vizinhança aberta para $q$ e $\varphi: U \longrightarrow \mathbb{R}^{n}$ um homeomorfismo. Vejamos que existe $t \in[0,1)$ tal que $\alpha([t, 1]) \subseteq U$. De fato, temos que $1 \in \alpha^{-1}(U) \subseteq[0,1]$, com $\alpha^{-1}(U)$ aberto em [0,1]. Então, existe $V \subseteq \mathbb{R}$ aberto tal que $\alpha^{-1}(U)=[0,1] \cap V$. Como $1 \in V$, existe $\delta>0$ tal que $(1-\delta, 1+\delta) \subseteq V$. Assim,

$$
(r, 1]=(1-\delta, 1+\delta) \cap[0,1] \subseteq \alpha^{-1}(U), \text { onde } r=\max \{1-\delta, 0\} \geq 0 .
$$

Logo, existe $r \geq 0$ tal que $(r, 1] \subseteq \alpha^{-1}(U)$ e, portanto, $\alpha((r, 1]) \subseteq U$. Dessa forma, considerando $t \in(r, 1)$, temos que existe $t \in[0,1)$ tal que $\alpha([t, 1]) \subseteq U$. Usando os mesmos argumentos para o caminho $\beta$, concluímos que existe $t^{\prime} \in(0,1]$ tal que $\beta\left(\left[0, t^{\prime}\right]\right) \subseteq U$.

Denotemos por $a:=\alpha(t) \neq q$ e $b:=\beta\left(t^{\prime}\right) \neq q$. Seja $\gamma:[0,1] \longrightarrow \mathbb{R}^{n}$ o caminho em $\mathbb{R}^{n}$ unindo $\varphi(a)$ a $\varphi(b)$ e tal que $\varphi(q) \notin \gamma([0,1])$. Consideremos o caminho

$$
\theta=\varphi^{-1} \circ \gamma:[0,1] \longrightarrow U
$$

$\operatorname{com} \theta(0)=a, \theta(1)=b$ e $q \notin \theta([0,1])$. Assim, obtemos o caminho em $M^{\prime}$

$$
\left(\left.\alpha\right|_{[0, t]}\right) *\left(\left.\theta\right|_{[0,1]}\right) *\left(\left.\beta\right|_{\left[t^{\prime}, 1\right)}\right):[0,1] \longrightarrow M-\{q\}
$$

unindo $x$ a $y$, onde $*$ denota a concatenação de caminhos. Segue que $M^{\prime}=M-\{q\}$ é conexa por caminhos, logo conexa.

Suponhamos agora que o resultado seja válido para qualquer subconjunto $F \subseteq M$, $\operatorname{com}|F|:=k<\infty$. Mostremos que $M-F^{\prime}$ é conexo, para $F^{\prime} \subseteq M$, com $\left|F^{\prime}\right|=|F|+1$. Considerando $F^{\prime}=\left\{q_{1}, \ldots, q_{k}, q_{k+1}\right\}$, temos por hipótese de indução que

$$
M^{\prime}:=M-\left\{q_{1}, \ldots, q_{k}\right\}
$$

é uma $n$-variedade topológica conexa por caminhos, onde $n \geq 2$. Portanto, segue do passo inicial da indução que

$$
M-F^{\prime}=M^{\prime}-\left\{q_{k+1}\right\}
$$

é uma $n$-variedade conexa por caminhos, logo conexa, como queríamos demonstar. 
Observação 2.1.25. A Proposição 2.1.24 não é verdade para espaços topológicos gerais, ou seja, se $X$ é um espaço topológico conexo por caminhos então não é verdade em geral que $X-F$ seja um espaço topológico conexo por caminhos, onde $F \subseteq M$ finito. Por exemplo, o espaço wedge $\mathbb{S}^{2} \vee \mathbb{S}^{2}:=\mathbb{S}^{2} \times\left\{x_{0}\right\} \cup\left\{x_{0}\right\} \times \mathbb{S}^{2}$, subespaço de $\mathbb{S}^{2} \times \mathbb{S}^{2}$, para algum ponto base $x_{0} \in \mathbb{S}^{2}$, não é uma variedade topológica e note que $\mathbb{S}^{2} \vee \mathbb{S}^{2}-\left\{\left(x_{0}, x_{0}\right)\right\}$ não é conexo por caminhos.

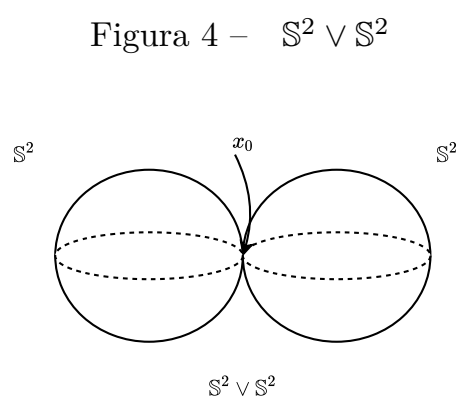

Fonte: Elaborada pelo autor.

Um outro exemplo é o grafo conexo por caminhos "Y", o qual não é uma 1-variedade topológica. Temos que $Y-\left\{y_{0}\right\}$ não é conexo por caminhos, onde $y_{0} \in$ "Y" não é um vértice bordo do grafo "Y". Este exemplo mostra uma das dificultadões em se trabalhar com grafos, os quais são os espaços topológicos mais usados em robótica (vide (KURLIN et al., 2012)).

\subsection{Espaços de configurações para variedades com bordo}

Nesta seção, mostraremos que o bordo de uma variedade topológica é irrelevante para os espaços de configurações, a menos de homotopia. Este fato foi afirmado por Bödigheimer, C.F. ; Cohen, F. e Taylor, L. em (BÖDIGHEIMER; COHEN; TAYLOR, 1989), Section 2, pg. 113.

Primeiramente, apresentamos o conceito de variedade com bordo. Pontos nestes espaços terão vizinhanças modeladas ou sobre subconjuntos abertos de $\mathbb{R}^{n}$ ou sobre subconjuntos abertos do $n$-dimensional semi-espaço superior fechado:

$$
\mathbb{H}^{n}:=\left\{\left(x_{1}, \ldots, x_{n}\right) \in \mathbb{R}^{n}: \quad x_{n} \geq 0\right\} \subset \mathbb{R}^{n}
$$

Definição 2.2.1. ((LEE, 2012), Chapter 1, pg. 25) Uma n-dimensional variedade topológica com bordo, ou simplesmente, uma n-variedade com bordo é um espaço topológico $M$ que é Hausdorff, segundo enumerável e satisfaz a seguinte condição: para todo $x \in M$, existe um subconjunto $U \subseteq M$ aberto em $M$, com $x \in U$, tal que $U$ é homeomorfo ou a um subconjunto aberto do $\mathbb{R}^{n}$ ou a um subconjunto aberto (relativo à topologia de 
sub-espaço) do $n$-dimensional semi-espaço superior fechado $\mathbb{H}^{n}$, considerando $\mathbb{H}^{n}$ munido da topologia de subespaço do $\mathbb{R}^{n}$ (equipado com a topologia usual). Um par $(U, \varphi)$, no qual $U \subset M$ é um subconjunto aberto, junto com uma aplicação $\varphi: U \longrightarrow V \subset \mathbb{R}^{n}$, que é um homeomorfismo sobre um subconjunto aberto $V$ de $\mathbb{R}^{n}$ ou de $\mathbb{H}^{n}$ será chamado uma carta para $M$.

Usaremos as notações $\operatorname{Fr}\left(\mathbb{H}^{n}\right)$ e $\operatorname{int}\left(\mathbb{H}^{n}\right)$ para denotar a fronteira e o interior (topológicos) de $\mathbb{H}^{n}$, respectivamente, considerando $\mathbb{H}^{n}$ como um subconjunto de $\mathbb{R}^{n}$. Quando $n>0$, isso significa:

$$
\begin{aligned}
& \left.\operatorname{Fr}\left(\mathbb{H}^{n}\right)=\left\{\left(x_{1}, \ldots, x_{n}\right) \in \mathbb{R}^{n}: x_{n}=0\right\} \quad \text { (homeomorfo a } \mathbb{R}^{n-1}\right) \\
& \left.\operatorname{int}\left(\mathbb{H}^{n}\right)=\left\{\left(x_{1}, \ldots, x_{n}\right) \in \mathbb{R}^{n}: x_{n}>0\right\} \quad \text { (homeomordo a } \mathbb{R}^{n}\right)
\end{aligned}
$$

No caso $n=0, \mathbb{H}^{0}=\mathbb{R}^{0}=\{0\}$, então $\operatorname{int}\left(\mathbb{H}^{0}\right)=\mathbb{H}^{0}$ e $\operatorname{Fr}\left(\mathbb{H}^{0}\right)=\emptyset$. Segue que 0dimensionais variedades com bordo não são diferentes de 0 -variedades. Se $M$ é uma $n$-variedade com bordo:

1. Chamaremos $(U, \varphi)$ uma carta interior se $\varphi(U)$ é um subconjunto aberto do $\mathbb{R}^{n}$ (o que inclui o caso de um subconjunto aberto de $\mathbb{H}^{n}$ que não intersecta $\operatorname{Fr}\left(\mathbb{H}^{n}\right)$. Um ponto $x \in M$ é chamado um ponto interior de $M$ se $x$ pertence ao domínio de alguma carta interior. O conjunto

$$
\operatorname{int} M:=\{x \in M: \quad x \text { é ponto interior de } M\}
$$

é chamado o interior da variedade $M$. Na Figura 5, $x$ representa um ponto interior da variedade $M$.

2. Chamaremos $(U, \varphi)$ uma carta de bordo se $\varphi(U)$ é um subconjunto aberto de $\mathbb{H}^{n}$ tal que $\varphi(U) \cap \operatorname{Fr}\left(\mathbb{H}^{n}\right) \neq \emptyset$. Um ponto $y \in M$ é chamado um ponto de bordo de $M$ se $y$ está no domínio de uma carta de bordo que leva $y$ em $\operatorname{Fr}\left(\mathbb{H}^{n}\right)$. O conjunto

$$
\partial M:=\{y \in M: \quad y \text { é ponto de bordo de } M\}
$$

é chamado o bordo da variedade $M$. Na Figura 5, y representa um ponto de bordo da variedade $M$.

Exemplo 2.2.2. O $n$-dimensional semi-espaço superior fechado $\mathbb{H}^{n}$ e o $n$-disco unitário $\mathbb{D}^{n}$ são $n$-variedades topológicas com bordo, assim como toda bola fechada do $\mathbb{R}^{n}$. Os intervalos fechados em $\mathbb{R}$ são 1-variedades com bordo.

Observação 2.2.3. Apesar do seu nome, variedades com bordo não são, em geral, variedades topológicas (no sentido da Definição 1.4.3), pois pontos de bordo não possuem vizinhanças localmente Euclideanas. Isso é uma consequência do Teorema da Invariância 
Figura 5 - Uma $n$-variedade com bordo

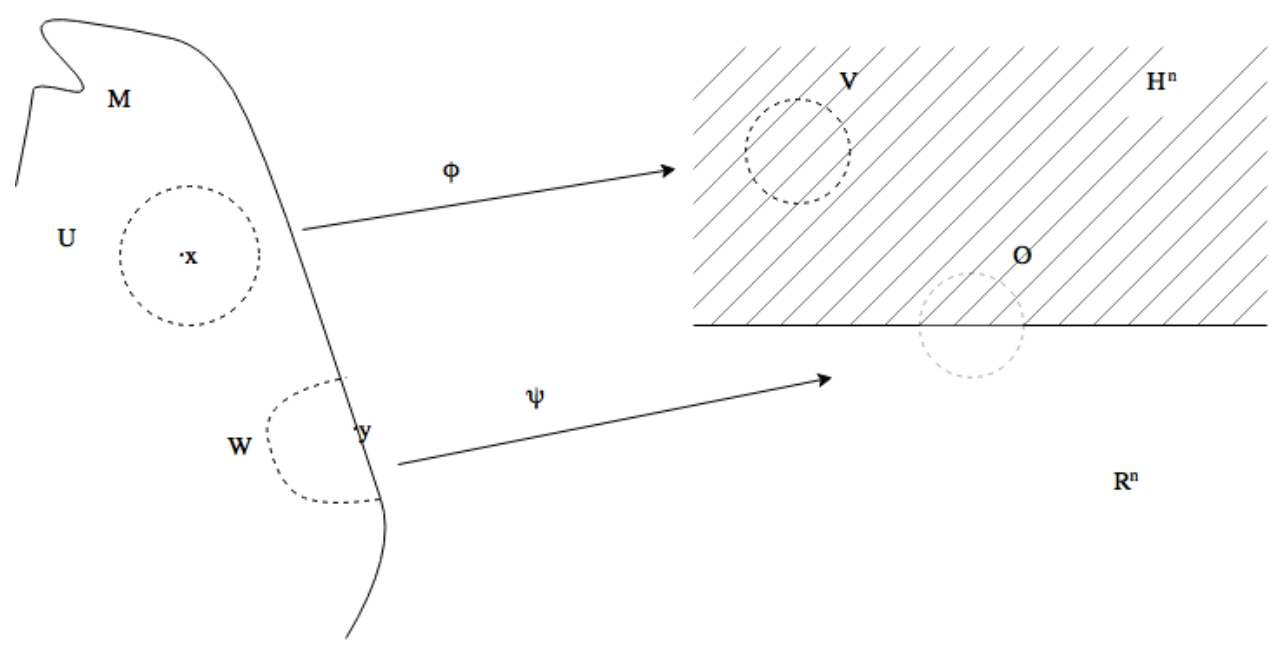

Fonte: Elaborada pelo autor.

do Bordo, o qual afirma que $\partial M$ e int $M$ são subconjuntos disjuntos cuja reunião é $M$ (vide (LEE, 2012), Theorem 1.37, pg. 26). Mais ainda, uma variedade com bordo, pode ter bordo vazio. Por outro lado, uma variedade topológica é também uma variedade topológica com bordo, cujo bordo é vazio. Assim, toda variedade topológica é uma variedade topológica com bordo. Mas uma variedade topológica com bordo $M$ será uma variedade topológica se, e somente se, $\partial M=\emptyset$ (vide (LEE, 2012), Proposition 1.38-(c)). Usaremos a frase: variedade topológica sem bordo, se quisermos enfatizar que estamos nos referindo a uma variedade topológica (no sentido original da Definição 1.4.3). E diremos: variedade topológica com ou sem bordo para nos referirmos a uma variedade topológica com bordo, se quisermos enfatizar que o seu bordo pode ser vazio. Portanto, o termo variedade topológica, sem nenhuma outra qualificação, sempre significará uma variedade topológica sem bordo. Se $M$ é uma $n$-variedade topológica com bordo, segue de ((LEE, 2012), Proposition 1.38-(a),(b),(d), pg. 27) que:

(i) int $M$ é um subconjunto aberto de $M$, o qual é uma $n$-variedade topológica sem bordo.

(ii) $\partial M$ é um subconjunto fechado de $M$, o qual é uma $(n-1)$-variedade sem bordo.

(iii) Se $n=0$, então $\partial M=\emptyset$ e $M$ é uma 0-variedade topológica sem bordo.

Definição 2.2.4. ((LEE, 2012), Chapter 1, pg. 27) Uma variedade fechada é uma variedade topológica compacta conexa e sem bordo. Uma variedade aberta é uma variedade topológica não compacta e sem bordo.

A seguir, apresentamos as principais ferramentas para esta seção. 
Definição 2.2.5. ((BROWN, 1962), Section II, pg. 332) Sejam $X$ um espaço topológico e $B$ um subconjunto de $X$. Dizemos que $B$ tem a propriedade do colarinho em $X$, se existe um homeomorfismo $f$ levando $B \times[0,1)$ sobre uma vizinhança aberta $V$ de $B$ tal que $f(b, 0)=b, \forall b \in B$.

Proposição 2.2.6. (Teorema da vizinhança colarinho)( (BROWN, 1962), Section IV, Theorem 2, pg. 339)) Seja $M$ uma variedade topológica com bordo, conexa e metrizável. Então, $\partial M$ tem a propriedade do colarinho em $M$, ou seja, existe um homeomorfismo $f$ levando $\partial M \times[0,1)$ sobre uma vizinhança aberta $V$ do bordo $\partial M$, tal que $f(b, 0)=$ $b, \forall b \in \partial M$.

Figura 6 - Vizinhança colarinho

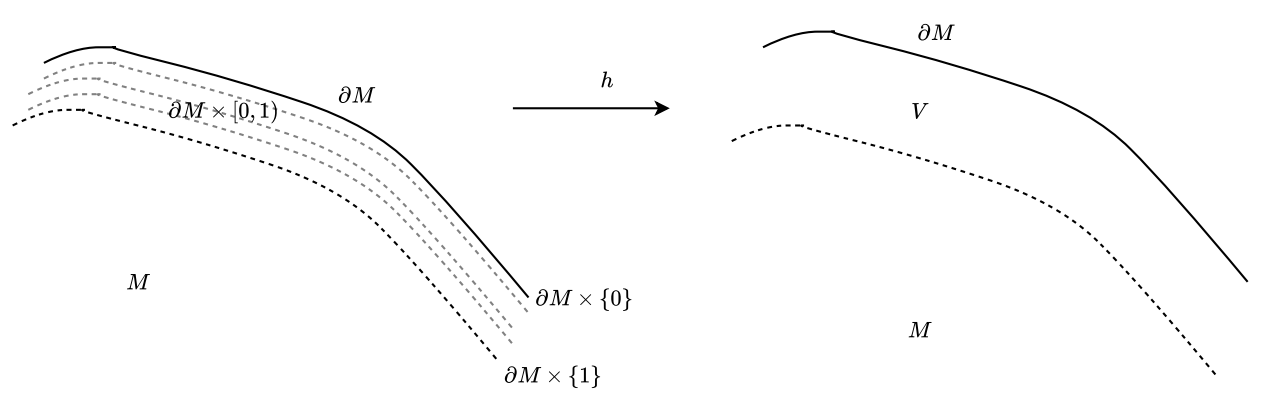

Fonte: Elaborada pelo autor.

Definição 2.2.7. Um espaço topológico $X$ é chamado um Retrato de uma Vizinhança Euclideana ou ENR se, para algum $n$, existe um mergulho $i: X \hookrightarrow \mathbb{R}^{n}$ tal que $i(X)$ é um retrato $^{1}$ de alguma vizinhança aberta em $\mathbb{R}^{n}$.

Observação 2.2.8. Seja $M$ uma variedade topológica compacta, com ou sem bordo. Então, $M$ é um Retrato de uma Vizinhança Euclideana (ENR). Em particular, $M$ é metrizável (vide (HATCHER, 2002), Appendix A, Corollary A.9, pg. 527).

Podemos considerar as seguintes versões alternativas da Definição 2.2 .5 e da Proposição 2.2.6, as quais serão usadas nesta seção.

Definição 2.2.9. Sejam $X$ um espaço topológico e $B$ um subconjunto de $X$. Dizemos que $B$ tem a propriedade do colarinho em $X$, se existe um homeomorfismo $h$ levando $B \times(-1,1]$ sobre uma vizinhança aberta $V$ de $B$, tal que $h(b, 1)=b, \forall b \in B$.

Proposição 2.2.10. (Teorema da vizinhança colarinho) Seja $M$ uma variedade topológica com bordo, compacta e conexa. Então, $\partial M$ possui a propriedade do colarinho em $M$, ou

$\overline{1}$ Dados $X$ um espaço topológico e $i: A \hookrightarrow X$ a inclusão de um subespaço $A \subset X$, dizemos que $A$ é um retrato de $X$, se existe uma função contínua $r: X \rightarrow A$ tal que $r \circ i=I d_{A}$. 
seja, existe um homeomorfismo $h$ levando $\partial M \times(-1,1]$ sobre uma vizinhança aberta $V$ do bordo $\partial M$ tal que $h(b, 1)=b, \forall b \in \partial M$.

Demonstração. Como $M$ é uma variedade topológica compacta, segue da Observação 2.2.8 que $M$ é metrizável. Assim, sendo $M$ uma variedade com bordo conexa e metrizável temos, pela Proposição 2.2.6, que existe um homeomorfismo $f: \partial M \times[0,1) \longrightarrow V \subseteq M$ levando $\partial M \times[0,1)$ sobre uma vizinhança aberta $V$ do bordo $\partial M$, tal que $f(b, 0)=b, \forall b \in \partial M$. Consideremos o homeomorfismo $g:(-1,1] \longrightarrow[0,1), \quad x \mapsto g(x):=-\frac{x}{2}+\frac{1}{2}$. Então,

$$
h:=f \circ(I d \times g): \partial M \times(-1,1] \longrightarrow V \subseteq M,
$$

é um homeomorfismo satisfazendo as condições desejadas.

Exemplo 2.2.11. Seja $\mathbb{H}^{n}$ o $n$-dimensional semi-espaço superior fechado. Então, $\partial \mathbb{H}^{n}$ tem a propriedade do colarinho em $\mathbb{H}^{n}$. Basta considerar o homeomorfismo

$$
h:=I d \times g: \partial \mathbb{H}^{n} \times(-1,1] \longrightarrow V \subseteq \mathbb{H}^{n},
$$

onde $g:(-1,1] \longrightarrow[0,1)$ é o homeomorfismo definido por: $\quad t \mapsto g(t):=-\frac{t}{2}+\frac{1}{2}$ e $V:=h\left(\partial \mathbb{H}^{n} \times(-1,1]\right)=\partial \mathbb{H}^{n} \times[0,1)$ é aberto em $\mathbb{H}^{n}$.

Proposição 2.2.12. Seja $M$ uma variedade topológica com bordo, compacta e conexa. Então, a inclusão $i: \operatorname{int} M \hookrightarrow M$ é uma equivalência de homotopia, com inversa homotópica que denotaremos por $p: M \longrightarrow \operatorname{int} M$ (ou seja, $H: i \circ p \simeq I d_{M}$ e $G: p \circ i \simeq I d_{\text {int } M}$ ). Além disso, $p$ é injetora e as homotopias $H$ e $G$ são isotopias.

Demonstração. Pela Proposição 2.2.10, $\partial M$ possui a propriedade do colarinho em $M$. Seja $h$ um homeomorfismo levando $\partial M \times(-1,1]$ em uma vizinhança aberta $V$ de $\partial M$ em $M$ e tal que $h(b, 1)=b, \forall b \in \partial M$. Consideremos o conjunto

$$
F:=M-h(\partial M \times(0,1]) \subseteq \operatorname{int} M
$$

o qual é fechado em $M$, pois $h(\partial M \times(0,1])$ é aberto em $V$, que é aberto em $M$.

Definimos a aplicação $p: M \longrightarrow$ int $M$ por:

$$
\forall y \in M, \quad p(y):= \begin{cases}h\left(x, \frac{1}{2} t\right), & \text { se } y=h(x, t), \quad 0 \leq t \leq 1 \\ y, & \text { se } y \in F\end{cases}
$$

Observemos que $h(\partial M \times[0,1])$ é um subconjunto fechado em $M$, pois $\partial M \times[0,1]$ é compacto e $h$ é contínua. Como as funções que definem $p$ são contínuas (mais ainda, injetoras) sobre os conjuntos fechados $h(\partial M \times[0,1])$ e $F$, respectivamente, e coincidem na intersecção:

$$
h(\partial M \times[0,1]) \cap F=h(\partial M \times\{0\}),
$$


Figura 7 - Vizinhança colarinho

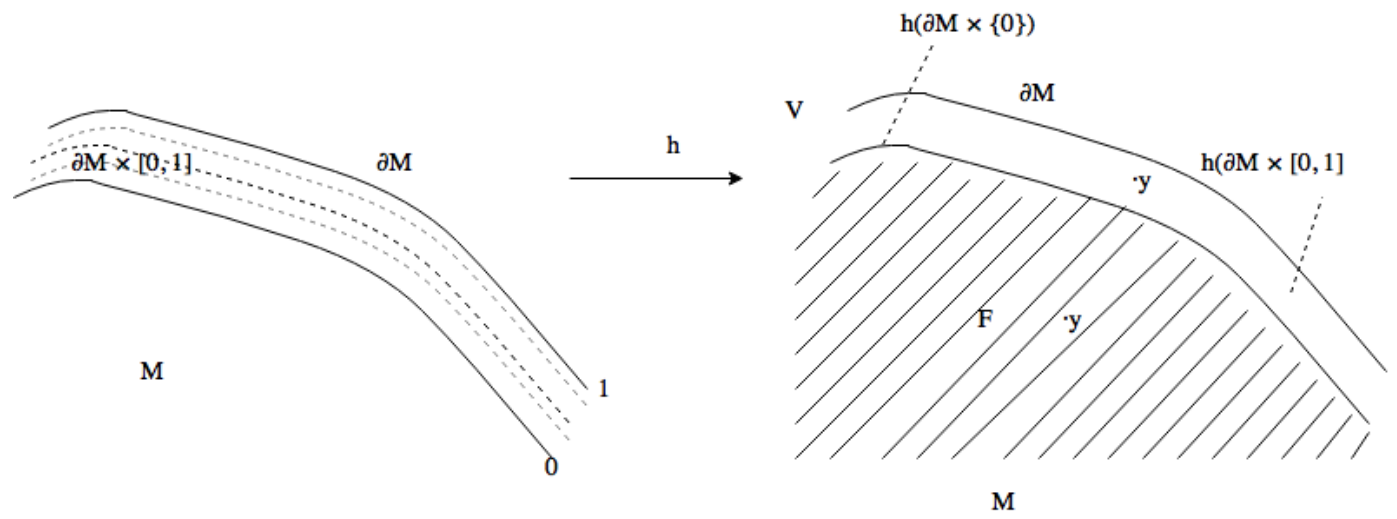

Fonte: Elaborada pelo autor.

segue que $p$ é bem definida, contínua e injetora. Mostremos que existe uma homotopia $H: M \times I \longrightarrow M$ entre $i \circ p$ e a aplicação identidade $I d_{M}$ tal que $H_{s}$ é injetora, para cada $s \in I$. Consideremos a aplicação $H: M \times I \longrightarrow M$ definida por:

$$
\forall y \in M, \quad \forall s \in I, \quad H(y, s):= \begin{cases}h\left(x,\left(\frac{1}{2}+\frac{s}{2}\right) t\right), & \text { se } y=h(x, t), \quad 0 \leq t \leq 1 \\ y, & \text { se } y \in F\end{cases}
$$

onde $\frac{1}{2}+\frac{s}{2}=l(s)$ é o comprimento do segmento de reta horizontal na altura $s$, como representado na Figura 8.

Figura 8 - Isotopia entre $i \circ p$ e $I d_{M}$.
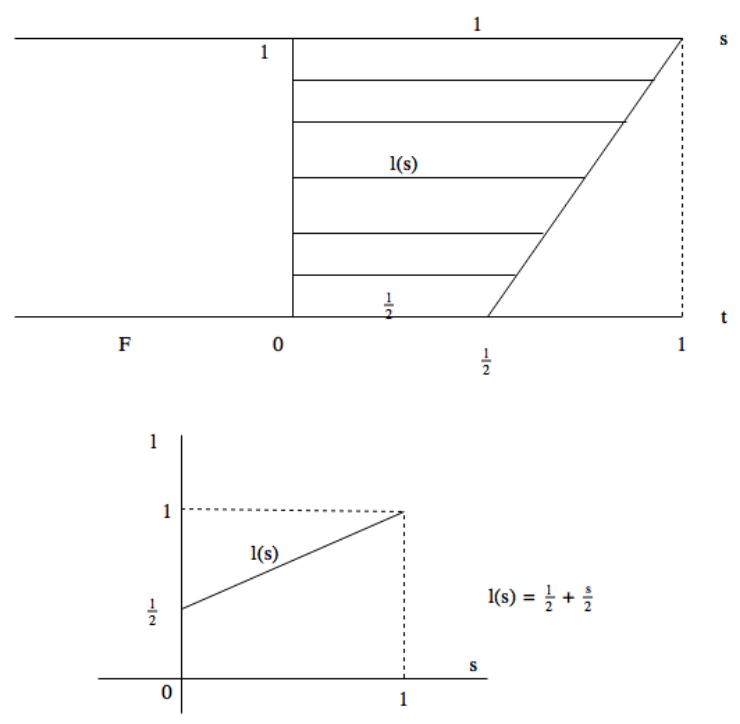

Fonte: Elaborada pelo autor.

Segue da definição de $H$ que $\forall y \in M, H(y, 0)=(i \circ p)(y)$ e $H(y, 1)=I d_{M}(y)$. Além disso, $H_{s}$ é injetora, para cada $s \in[0,1]$. Portanto, $H$ é uma isotopia entre $i \circ p$ e $I d_{M}$. Note que, restringindo $H$ a $(i n t M) \times I$, obtemos uma isotopia $G$ entre $p \circ i$ e $I d_{\text {int } M}$. 


\section{O bordo de uma variedade é irrelevante para os espaços de configura- ções, a menos de homotopia}

Apresentamos a seguir o resultado principal desta seção.

Teorema 2.2.13. ((BÖDIGHEIMER; COHEN; TAYLOR, 1989), Section 2, pg. 113) Seja $M$ uma variedade topológica com bordo, compacta e conexa, com $k \geq 1$. Então, a inclusão $i: \operatorname{int} M \hookrightarrow M$ induz equivalências de homotopia:

$$
F(i): F(\text { int } M, k) \hookrightarrow F(M, k) \text { e } S F(i): S F(\text { int } M, k) \hookrightarrow S F(M, k)
$$

Demonstração. Pela Observação 2.1.11, para cada $k \geq 1$, temos um funtor covariante:

$$
F(-, k): \mathfrak{T o p}_{i} \longrightarrow \mathfrak{T o p}_{i}
$$

Assim, com as mesmas notações da Proposição 2.2.12,

$$
F(i): F(\text { int } M, k) \hookrightarrow F(M, k) \text { e } F(p): F(M, k) \longrightarrow F(\text { int } M, k)
$$

são funções contínuas e injetoras. Além disso, as isotopias

$$
G: \operatorname{int} M \times I \longrightarrow \operatorname{int} M \text { e } H: M \times I \longrightarrow M
$$

entre $p \circ i, \quad I d_{i n t M}$ e $i \circ p, \quad I d_{M}$, respectivamente, induzem homotopias:

$$
F(G): F(\text { int } M, k) \times I \longrightarrow F(\text { int } M, k) \text { e } F(H): F(M, k) \times I \longrightarrow F(M, k),
$$

entre entre $F(p \circ i), \quad F\left(I d_{i n t M}\right)$ e $F(i \circ p), \quad F\left(I d_{M}\right)$, respectivamente, ou seja:

$$
\begin{gathered}
F(p) \circ F(i)=F(p \circ i) \stackrel{F(G)}{\simeq} F\left(I d_{i n t M}\right)=I d_{F(i n t M, k)}, \\
F(i) \circ F(p)=F(i \circ p) \stackrel{F(H)}{\simeq} F\left(I d_{M}\right)=I d_{F(M, k)} .
\end{gathered}
$$

Portanto, $F(i)$ é uma equivalência de homotopia, com inversa homotópica $F(p)$. Analogamente, mostra-se que o resultado é válido para o caso não ordenado.

Observação 2.2.14. O Teorema 2.2.13 mostra que o bordo de uma variedade topológica compacta e conexa é irrelevante para os espaços de configurações, a menos de homotopia. Isto significa dizer que é suficiente calcular os espaços de configurações para variedades topológicas sem bordo.

Exemplo 2.2.15. Como $\mathbb{S}^{n} \times \mathbb{R}$ é homeomorfo a $\mathbb{S}^{n} \times(0,1)$, segue da Proposição 2.1.13(2) que $F\left(\mathbb{S}^{n} \times \mathbb{R}, k\right)$ é homeomorfo a $F\left(\mathbb{S}^{n} \times(0,1), k\right), \forall k \geq 1$. Por outro lado, pelo Teorema 2.2.13, os espaços de configurações

$$
F(\underbrace{\mathbb{S}^{n} \times(0,1)}_{i n t\left(\mathbb{S}^{n} \times[0,1]\right)}, k) \text { e } F\left(\mathbb{S}^{n} \times[0,1], k\right)
$$


possuem o mesmo tipo de homotopia, $\forall k \geq 1$. Portanto, concluímos que $\forall k \geq 1$,

$$
F\left(\mathbb{S}^{n} \times \mathbb{R}, k\right) \text { e } F\left(\mathbb{S}^{n} \times[0,1], k\right)
$$

possuem o mesmo tipo de homotopia. Note que $\mathbb{S}^{n} \times \mathbb{R}$ é uma variedade não compacta e $\mathbb{S}^{n} \times[0,1]$ é uma variedade compacta. De maneira análoga, mostra-se que o resultado é válido para o caso não ordenado.

Exemplo 2.2.16. Seja $\mathbb{H}^{n}$ o $n$-dimensional semi-espaço superior fechado. Com as mesmas notações do Exemplo 2.2.11, como $\partial \mathbb{H}^{n}$ possui a propriedade do colarinho em $\mathbb{H}^{n}$, seja $h$ o homeomorfismo levando $\partial \mathbb{H}^{n} \times(-1,1]$ sobre a vizinhança aberta $V:=h\left(\partial \mathbb{H}^{n} \times(-1,1]\right)=$ $\partial \mathbb{H}^{n} \times[0,1)$ do bordo $\partial \mathbb{H}^{n}$, tal que $h(b, 1)=b, \forall b \in \partial \mathbb{H}^{n}$. Então,

$$
h\left(\partial \mathbb{H}^{n} \times[0,1]\right)=\partial \mathbb{H}^{n} \times[0,1 / 2],
$$

é fechado em $\mathbb{H}^{n}$. Assim, podemos usar os mesmos argumentos apresentados na demonstração da Proposição 2.2.12 para a variedade não compacta $\mathbb{H}^{n}$. Note que neste caso, não precisamos da hipótese de compacidade para concluir que a inclusão $i$ : int $\mathbb{H}^{n} \hookrightarrow \mathbb{H}^{n}$ é uma equivalência de homotopia. Dessa forma, usando as propriedades funtoriais de $F(-, k): \mathfrak{T o p}_{i} \longrightarrow \mathfrak{T o p}_{i}$, concluímos que $F\left(\right.$ int $\left.\mathbb{H}^{n}, k\right)$ tem o mesmo tipo de homotopia de $F\left(\mathbb{H}^{n}, k\right), \forall k \geq 1$. Por outro lado, como $i n t \mathbb{H}^{n}$ é homeomorfo ao $\mathbb{R}^{n}$, temos da Proposição 2.1.13-(2) que $F\left(\right.$ int $\left.\mathbb{H}^{n}, k\right)$ é homeomorfo a $F\left(\mathbb{R}^{n}, k\right), \forall k \geq 1$. Portanto,

$$
F\left(\mathbb{H}^{n}, k\right) \simeq F\left(\mathbb{R}^{n}, k\right), \quad \forall k \geq 1
$$

Similarmente, mostra-se que

$$
S F\left(\mathbb{H}^{n}, k\right) \simeq S F\left(\mathbb{R}^{n}, k\right), \quad \forall k \geq 1
$$



CAPÍTULO

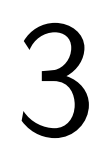

\section{ESPAÇO DE CONFIGURAÇÕES PARA SUPERFÍCIES}

Nesta capítulo, apresentamos os resultados e as notações básicas para o estudo dos espaços de configurações para superfícies ${ }^{1}$. Tais espaços serão interessantes para definir os grupos de tranças e os grupos de tranças puras de uma superfície.

\subsection{Fibração de Fadell e Neuwirth}

Definição 3.1.1. Para um espaço topológico $M$, consideremos $T o p(M)$ o espaço dos homeomorfismos $h: M \longrightarrow M$, o qual é munido da topologia compacta-aberta (vide Definições 1.2 .1 e 1.2.10). (Top $(M)$, o ) é um grupo com a operação o de composição de funções, chamado o grupo dos homeomorfismos de $M$.

Definição 3.1.2. ((FADELL; HUSSEINI, 2001), Part I, Section I.1, pg. 6) Seja $M$ uma variedade topológica. Um homeomorfismo $h: M \longrightarrow M$ é chamado estável, se existe um subconjunto fechado próprio $F \subset M$, tal que $h(x)=x, \quad \forall x \in M-F$.

Exemplo 3.1.3. Como exemplo de um homeomorfismo estável temos a aplicação identidade $I d_{M}: M \longrightarrow M$, com $F=\emptyset$.

Observação 3.1.4. Os homeomorfismos estáveis geram um subgrupo de $T o p(M)$, o qual denotaremos por $\operatorname{Top}_{s}(M)$. Assim, um elemento $h$ do subgrupo $\operatorname{Top}_{s}(M)$ é da forma:

$$
h=h_{1}^{m_{1}} \circ \cdots \circ h_{k}^{m_{k}}, \text { com } h_{i} \text { homeomorfismo estável e } m_{i} \in \mathbb{Z} .
$$

Definição 3.1.5. Para cada $x=\left(x_{1}, \ldots, x_{k}\right) \in F(M, k)$, consideremos o conjunto:

$$
Q_{r}^{x}:=\left\{x_{1}, \ldots, x_{r}\right\}, \text { onde } 1 \leq r \leq k .
$$

1 Estaremos considerando uma superfície (topológica) como sendo uma 2-dimensional variedade topológica conexa 
Quando $x$ é o ponto base $q=\left(q_{1}, \ldots, q_{k}\right)$, o conjunto $Q_{r}^{q}$ será denotado simplesmente por $Q_{r}$. Por convenção, $Q_{0}$ denotará o conjunto vazio $\emptyset$.

Observação 3.1.6. ((FADELL; HUSSEINI, 2001), Part I, Section I.1, pg. 6) Note que o subgrupo $\operatorname{Top}_{s}(M)$ age naturalmente pela ação diagonal sobre o espaço de configurações associado $F(M, k)$, como segue:

$$
\varphi: \operatorname{Top}_{s}(M) \times F(M, k) \longrightarrow F(M, k), \text { onde } \varphi\left(h,\left(x_{1}, \ldots, x_{k}\right)\right):=\left(h\left(x_{1}\right), \ldots, h\left(x_{k}\right)\right) .
$$

Esta ação induz um homomorfismo de grupos, chamado uma representação:

$$
\rho: \operatorname{Top}_{s}(M) \longrightarrow \operatorname{Top}(F(M, k)) \text { dado por } \rho(h)\left(x_{1}, \ldots, x_{k}\right):=\left(h\left(x_{1}\right), \ldots, h\left(x_{k}\right)\right),
$$

o qual é injetor, ou seja, a ação é fiel. De fato, para quaisquer $h, f \in \operatorname{Top}_{s}(M)$ tais que $\rho(h)=\rho(f)$, temos que para cada $m \in M$ :

$$
\begin{aligned}
\left(h(m), h\left(m_{2}\right), \ldots, h\left(m_{k}\right)\right) & =\rho(h)\left(m, m_{2}, \ldots, m_{k}\right) \\
& =\rho(f)\left(m, m_{2}, \ldots, m_{k},\right) \\
& =\left(f(m), f\left(m_{2}\right), \ldots, f\left(m_{k}\right)\right)
\end{aligned}
$$

onde $m_{2}, \ldots, m_{k} \in M$ são escolhidos de tal forma que $\left(m, m_{2}, \ldots, m_{k}\right) \in F(M, k)$. Logo, $h(m)=f(m), \forall m \in M$, ou seja, $h=f$. Assim, Top $(M)$ é isomorfo ao subgrupo $\rho\left(\operatorname{Top}_{s}(M)\right) \subset T o p(F(M, k))$. Portanto, $\operatorname{Top}_{s}(M)$ é naturalmente um subgrupo do grupo dos homeomorfismos de $F(M, k), \forall k \geq 1$.

Definição 3.1.7. ((FADELL; HUSSEINI, 2001), Part I, Section I.1, pg. 6) Consideremos

$$
\operatorname{Top}_{s r}(M):=\left\{h \in \operatorname{Top}_{s}(M): \quad h\left(q_{i}\right)=q_{i}, \quad \forall 1 \leq i \leq r\right\}
$$

o qual é um subgrupo de $\operatorname{Top}_{s}(M)$.

Definição 3.1.8. Sejam $\mathbb{D}^{m}=\left\{\left(x_{1}, \ldots, x_{m}\right) \in \mathbb{R}^{n} ; \sum x_{i}^{2} \leq 1\right\}$ o $n$-disco unitário fechado em $\mathbb{R}^{m}$ e denotemos por $\mathbb{B}^{m}$ o seu interior. Consideremos o subconjunto:

$$
G_{0}\left(\mathbb{D}^{m}\right):=\left\{h \in T o p\left(\mathbb{D}^{m}\right): \quad h(x)=x, \quad \forall x \in \partial \mathbb{D}^{m}=\mathbb{S}^{m-1}\right\}
$$

Temos que $I d_{\mathbb{D}^{m}} \in G_{0}\left(\mathbb{D}^{m}\right)$, assim $G_{0}\left(\mathbb{D}^{m}\right) \neq \emptyset$. Além disso, se $h, f \in G_{0}\left(\mathbb{D}^{m}\right)$, então $h \circ f^{-1} \in T o p\left(\mathbb{D}^{m}\right) \quad$ e satisfaz $\left(h \circ f^{-1}\right)(x)=h(x)=x$, para qualquer $x \in \partial \mathbb{D}^{m}$. Portanto, $G_{0}\left(\mathbb{D}^{m}\right)$ é um subgrupo de $T o p\left(\mathbb{D}^{m}\right)$, o qual será equipado com a topologia compacto aberta.

Antes de enunciarmos o Teorema de Fadell e Neuwirth, um dos teoremas principais para os espaços de configurações, apresentamos o resultado a seguir, o qual será a base para a prova deste Teorema. 
Lema 3.1.9. ((FADELL; HUSSEINI, 2001), Lemma 1.1, pg. 6) Existe uma aplicação contínua $\gamma: \mathbb{B}^{m} \longrightarrow G_{0}\left(\mathbb{D}^{m}\right)$ satisfazendo as seguintes propriedades:

(i) $\gamma(x)(x)=0, \quad \forall x \in \mathbb{B}^{m}$.

(ii) $\gamma(x)(y)=y, \quad \forall y \in \partial \mathbb{D}^{m}$.

Demonstração. Sejam $g: \mathbb{B}^{m} \longrightarrow \mathbb{R}^{m}$ e $h: \mathbb{R}^{m} \longrightarrow \mathbb{B}^{m}$ definidas como segue:

$$
\begin{gathered}
g(y)=\frac{y}{1-|y|}, \quad y \in \mathbb{B}^{m} \mathrm{e} \\
h(x)=\frac{x}{1+|x|}, \quad x \in \mathbb{R}^{m} .
\end{gathered}
$$

Note que $g$ e $h$ são homeomorfismos com $g^{-1}=h$. Definimos $\gamma^{\prime}: \mathbb{B}^{m} \times \mathbb{D}^{m} \longrightarrow \mathbb{D}^{m}$ por:

$$
\gamma^{\prime}(x, y)= \begin{cases}h(g(y)-g(x)), & \forall y \in \mathbb{B}^{m}, \\ y, & \forall y \in \partial \mathbb{D}^{m}\end{cases}
$$

Temos que $\gamma^{\prime}$ é contínua, pois para qualquer $y_{0} \in \partial \mathbb{D}^{m}$ fixado e para todo $x \in \mathbb{B}^{m}$ :

$$
\begin{aligned}
\lim _{y \rightarrow y_{0}} h(g(y)-g(x)) & =\lim _{y \rightarrow y_{0}} \frac{g(y)-g(x)}{1+|g(y)-g(x)|} \\
& =\lim _{y \rightarrow y_{0}} \frac{\frac{y}{1-|y|}-\frac{x}{1-|x|}}{1+\left|\frac{y}{1-|y|}-\frac{x}{1-|x|}\right|} \\
& =\lim _{y \rightarrow y_{0}} \frac{(1-|x|) y-(1-|y|) x}{(1-|y|)(1-|x|)+|(1-|x|) y-(1-|y|) x|} \\
& =y_{0} .
\end{aligned}
$$

Além disso, para cada $x \in \mathbb{B}^{m}$ fixado, a aplicação contínua

$$
\gamma_{x}^{\prime}: \mathbb{D}^{m} \longrightarrow \mathbb{D}^{m}, \quad y \mapsto \gamma_{x}^{\prime}(y):=\gamma^{\prime}(x, y)
$$

é um homeomorfismo, desde que ela é uma bijeção contínua e $\mathbb{D}^{m}$ é um espaço compacto Hausdorff. Além disso, para cada $x \in \mathrm{B}^{m}, \gamma_{x}^{\prime}$ satisfaz:

$$
\gamma_{x}^{\prime}(y)=\gamma^{\prime}(x, y)=y, \quad \forall y \in \partial \mathbb{D}^{m}=\mathbb{S}^{m-1}
$$

Assim,

$$
\gamma_{x}^{\prime} \in G_{0}\left(\mathbb{D}^{m}\right)
$$

Usando a aplicação associação²:

$$
\varphi: \operatorname{Map}\left(\mathbb{B}^{m} \times \mathbb{D}^{m}, \mathbb{D}^{m}\right) \longrightarrow \operatorname{Map}\left(\mathbb{B}^{m}, \operatorname{Map}\left(\mathbb{D}^{m}, \mathbb{D}^{m}\right)\right),
$$

2 Vide Definição 1.2.15. 
consideramos a aplicação $\gamma: \mathbb{B}^{m} \longrightarrow G_{0}\left(\mathbb{D}^{m}\right)$ definida por

$$
\gamma:=\varphi\left(\gamma^{\prime}\right) \in \operatorname{Map}\left(\mathbb{B}^{m}, \operatorname{Map}\left(\mathbb{D}^{m}, \mathbb{D}^{m}\right)\right)
$$

a qual é bem definida, pois para cada $x \in \mathbb{B}^{m}, \gamma(x)=\gamma_{x}^{\prime}$ (pela definição de $\varphi$ ) e de (3.1.1), obtemos $\gamma(x) \in G_{0}\left(\mathbb{D}^{m}\right)$, para cada $x \in \mathbb{B}^{m}$. Além disso, $\gamma$ satisfaz as propriedades:

(a) Para cada $x \in \mathbb{B}^{m}$ :

$$
\begin{aligned}
\gamma(x)(x) & =\gamma^{\prime}(x, x) \\
& =h(g(x)-g(x)) \\
& =h(0) \\
& =0
\end{aligned}
$$

(b) Para cada $x \in \mathbb{B}^{m}$ e para cada $y \in \partial \mathbb{D}^{m}=\mathbb{S}^{m-1}$ :

$$
\begin{aligned}
\gamma(x)(y) & =\gamma^{\prime}(x, y) \\
& =y
\end{aligned}
$$

Figura 9 - Disco fechado numa variedade

$M$

$\mathbb{R}^{m}$
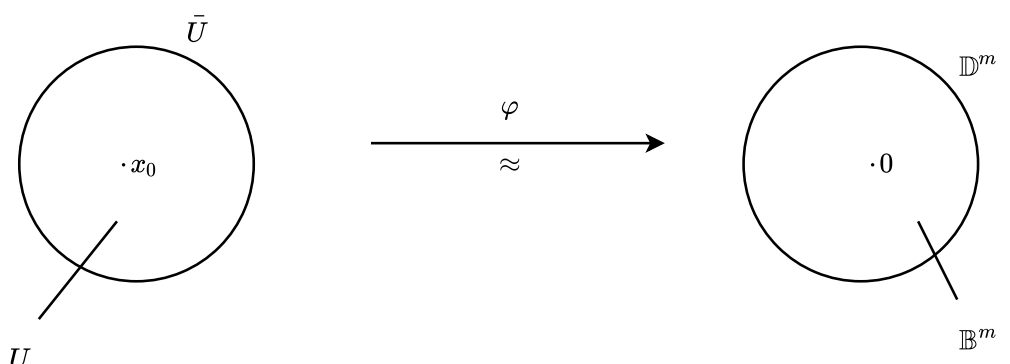

Fonte: Elaborada pelo autor.

Observação 3.1.10. Para uma $m$-variedade topológica $M$, fixe $x_{0} \in M$ e escolha $U$ um subconjunto aberto próprio de $M, \operatorname{com} x_{0} \in U$, cujo fecho $\bar{U}$ seja homeomorfo a $\mathbb{D}^{m}$; mediante um homeomorfismo $\varphi: \bar{U} \longrightarrow \mathbb{D}^{m}$; de tal forma que $\mathbb{B}^{m}$ corresponde a $U$, ou seja, $\varphi(U)=\mathbb{B}^{m}$ e $\varphi\left(x_{0}\right)=0$. O conjunto $\bar{U}$ é chamado uma vizinhança de disco ou disco fechado em $M$, para $x_{0} \in M$.

Com as mesmas notações da Observação 3.1.10, consideramos o resultado a seguir, o qual é uma consequência do Lema 3.1.9. 
Corolário 3.1.11. ((FADELL; HUSSEINI, 2001), Corollary 1.1, pg. 7) Existe uma aplicação contínua $\gamma^{*}: U \longrightarrow T o p_{s}(M)$ com as seguintes propriedades:

(i) $\gamma^{*}(x)(x)=x_{0}, \quad \forall x \in U$.

(ii) $\gamma^{*}(x)(y)=y, \quad \forall x \in U$ e $\forall y \in M-U$.

Demonstração. Primeiramente, definimos a aplicação:

$$
\theta: G_{0}\left(\mathbb{D}^{m}\right) \longrightarrow \operatorname{Top}_{s}(M)
$$

a qual leva $h \in G_{0}\left(\mathbb{D}^{m}\right)$ em $\theta(h)$, onde $\theta(h)$ é definido como segue:

$$
\theta(h)(y)= \begin{cases}\left(\varphi^{-1} \circ h \circ \varphi\right)(y), & \text { quando } y \in \bar{U} . \\ y, & \text { quando } y \in M-U .\end{cases}
$$

Note que as duas funções usadas para definir $\theta(h)$ são homeomorfismos nos fechados $\bar{U}$ e $M-U$, respectivamente. Além disso, para $y$ na interseção $\bar{U} \cap(M-U)$,

$$
\varphi^{-1} \circ h \circ \underbrace{\varphi(y)}_{\in \partial \mathbb{D}^{m}}=\varphi^{-1} \circ \varphi(y)=y .
$$

Então, o Lema da Colagem implica que $\theta(h)$ é contínua. Na verdade, $\theta(h)$ é um homeomorfismo estável. Assim, $\theta$ fica bem definida. A continuidade de $\theta$ segue de resultados relativos à topologia compacta aberta (Seção 1.2), como segue. Para $(K, W) \subseteq \operatorname{Top}_{s}(M)$ aberto sub-básico, temos:

$$
\theta^{-1}(K, W)=\theta^{-1}(K \cap \bar{U}, W) \cap \theta^{-1}(K \cap(M-U), W)
$$

pois

$$
(K, W)=(K \cap \bar{U}, W) \cap(K \cap(M-U), W) .
$$

De fato,

$$
\begin{aligned}
f \in(K, W) & \Longleftrightarrow f(K) \subseteq W \\
& \Longleftrightarrow f(K \cap M) \subseteq W \\
& \Longleftrightarrow f(K \cap(\bar{U} \cup(M-U))) \subseteq W \\
& \Longleftrightarrow f((K \cap \bar{U}) \cup(K \cap(M-U))) \subseteq W \\
& \Longleftrightarrow f(K \cap \bar{U}) \subseteq W \text { e } f(K \cap(M-U)) \subseteq W \\
& \Longleftrightarrow f \in(K \cap \bar{U}, W) \text { e } f \in(K \cap(M-U), W) \\
& \Longleftrightarrow f \in(K \cap \bar{U}, W) \cap(K \cap(M-U), W) .
\end{aligned}
$$

Note ainda que:

$$
\theta^{-1}(K \cap \bar{U}, W)=(\varphi(K \cap \bar{U}), \varphi(W \cap U)) \text { e } \theta^{-1}(K \cap(M-U), W)=\emptyset \text { ou } G_{0}\left(\mathbb{D}^{m}\right) .
$$

De fato, 
(a)

$$
\begin{aligned}
h \in \theta^{-1}(K \cap \bar{U}, W) & \Longleftrightarrow \theta(h) \in(K \cap \bar{U}, W) \\
& \Longleftrightarrow \theta(h)(K \cap \bar{U}) \subseteq W \\
& \Longleftrightarrow h(\varphi(K \cap \bar{U}) \subseteq \varphi(W \cap U) \\
& \Longleftrightarrow h \in(\varphi(K \cap \bar{U}), \varphi(W \cap U)) .
\end{aligned}
$$

(b) Se $\theta^{-1}(K \cap(M-U), W) \neq \emptyset$, vejamos que $\theta^{-1}(K \cap(M-U), W)=G_{0}\left(\mathbb{D}^{m}\right)$. Pelo fato que $\theta^{-1}(K \cap(M-U), W) \neq \emptyset$, obtemos $K \cap(M-U) \subseteq W$. Agora, para qualquer $h \in G_{0}\left(\mathbb{D}^{m}\right)$, temos:

$$
\theta(h)(K \cap(M-U))=K \cap(M-U) \subseteq W,
$$

$\operatorname{logo} h \in \theta^{-1}(K \cap(M-U), W)$. Assim, $\theta^{-1}(K \cap(M-U), W)=G_{0}\left(\mathbb{D}^{m}\right)$.

Agora, considerando a aplicação $\gamma: \mathbb{B}^{m} \longrightarrow G_{0}\left(\mathbb{D}^{m}\right)$ obtida no Lema 3.1.9, definimos $\gamma^{*}: U \longrightarrow \operatorname{Top}_{s}(M)$ como sendo a seguinte composição:

$$
U \stackrel{\varphi}{\longrightarrow} \mathbb{B}^{m} \stackrel{\gamma}{\longrightarrow} G_{0}\left(\mathbb{D}^{m}\right) \stackrel{\theta}{\longrightarrow} \operatorname{Top}_{s}(M) \text {, ou seja, } \gamma^{*}:=\theta \circ \gamma \circ \varphi .
$$

Assim, $\gamma^{*}$ é contínua e, para todo $x \in U$, denotando $\varphi(x)=y \in \mathbb{B}^{m}$, temos:

$$
\begin{aligned}
\gamma^{*}(x)(x) & =(\theta \circ \gamma) \circ \underbrace{\varphi(x)}_{:=y}(x) \\
& =\theta(\gamma(y))(x) \\
& =\varphi^{-1} \circ \gamma(y) \circ \underbrace{\varphi(x)}_{y} \\
& =\varphi^{-1}(\underbrace{\gamma(y)(y)}_{0}) \\
& =x_{0} .
\end{aligned}
$$

Analogamente, para $y \in M-U$ :

$$
\begin{aligned}
\gamma^{*}(x)(y) & =\theta \circ \gamma \circ \underbrace{\varphi(x)}_{:=z}(y) \\
& =\theta(\gamma(z))(y) \\
& =y .
\end{aligned}
$$

A seguir, consideramos o conceito de fibrados localmente triviais. Informalmente, um fibrado localmente trivial é um espaço que localmente se comporta como um espaço produto (no mesmo sentido em que uma variedade é localmente Euclideana), mas que globalmente pode possuir uma estrutura topológica diferente. Especificamente, a similaridade entre um espaço $E$ e um espaço produto $B \times F$ é definida usando-se uma aplicação contínua e sobrejetora $p: E \rightarrow B$, a qual localmente se comporta como uma projeção, como segue. 
Definição 3.1.12. ((AGUILAR; GITLER; PRIETO, 2002), Definition 4.5.1. pg. 126) Uma função contínua e sobrejetora $p: E \longrightarrow B$ é chamada um fibrado localmente trivial ${ }^{3}$ se para todo ponto $b \in B$, existem um aberto $U \subset B$, com $b \in U$, e um homeomorfismo $\varphi_{U}: U \times F \longrightarrow p^{-1}(U)$ satisfazendo a condição que a composição:

$$
U \times F \stackrel{\varphi_{U}}{\longrightarrow} p^{-1}(U) \stackrel{p_{U}}{\longrightarrow} U
$$

é a projeção no primeiro fator, ou seja,

$$
\left(p_{U} \circ \varphi_{U}\right)(x, y)=P_{1}(x, y)=x, \forall(x, y) \in U \times F
$$

onde $p_{U}=\left.p\right|_{p^{-1}(U)}: p^{-1}(U) \rightarrow U$ e $P_{1}$ é a projeção sobre $U$.

Observação 3.1.13. Se $p: E \rightarrow B$ é um fibrado localmente trivial, então $E$ é chamado o espaço total, $B$ o espaço base e, para cada $b \in B$, o espaço $F_{b}:=p^{-1}(b)$, homeomorfo a $F$, é chamado a fibra sobre $b$ (todas as fibras são homeomorfas).

Exemplo 3.1.14. Se considerarmos $U=B$, ou seja, se $E \cong B \times F$, então $p: E \longrightarrow B$ é chamada um fibrado trivial. Em particular, se $E=B \times F$, então $p$ (a projeção sobre $B$ ) é um fibrado trivial.

Exemplo 3.1.15. ((HATCHER, 2002), Chapter 4, Section 4.2, pg. 377) Toda aplicação de recobrimento $p: E \longrightarrow B$, sobre um espaço base conexo $B$, é um fibrado localmente trivial.

Observação 3.1.16. A fibração de Fadell e Neuwirth é a ferramenta central no estudo da topologia e geometria dos espaços de configurações. Em topologia, uma fibração é uma generalização da noção de fibrado localmente trivial. No caso de uma fibração, as fibras não precisam ser o mesmo espaço, mas elas serão homotopicamente equivalentes. Fibrações não possuem necessariamente a estrutura local de um espaço produto que define um fibrado, mas uma fibração deve satisfazer uma condição adicional: a Propriedade do Levantamento de Homotopia.

Definição 3.1.17. ((DAVIS; KIRK, 2001), Definition 6.7, pg. 115) Sejam $E, B, X$ espaços topológicos, $p: E \rightarrow B$ uma aplicação contínua e $I=[0,1]$. Dizemos que $p$ possui a Propriedade do Levantamento de Homotopia (P.L.H) com respeito ao espaço $X$ :

(i) se para qualquer homotopia $F: X \times I \rightarrow B$,

(ii) e se para toda função contínua $\tilde{f}_{0}: X \rightarrow E$, tal que $\tilde{f}_{0}$ é um levantamento de $f_{0}=\left.F\right|_{X \times\{0\}}$, ou seja, $f_{0}=p \circ \tilde{f}_{0}$, com $X$ homeomorfo a $X \times\{0\}$,

3 Ou ainda, um fibrado com fibra F, como em (HATCHER, 2002), Chapter 4, Section 4.2, pg. 376. 
existe uma homotopia $\tilde{F}: X \times I \rightarrow E$ que é um levantamento de $F$, ou seja, $F=p \circ \tilde{F}$, satisfazendo $\tilde{f}_{0}=\left.\tilde{F}\right|_{X \times\{0\}}$.
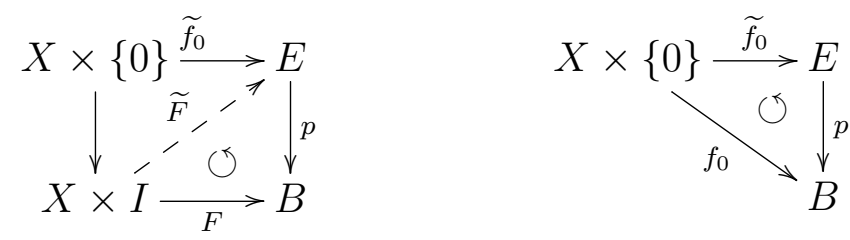

Definição 3.1.18. ((DAVIS; KIRK, 2001), Definition 6.7, pg. 115) Uma função contínua $p: E \rightarrow B$ satisfazendo a Propriedade do Levantamento de Homotopia (P.L.H) com respeito a qualquer espaço topológico $X$ é chamada uma fibração de Hurewicz ou simplesmente uma fibração.

Observação 3.1.19. Se $p: E \rightarrow B$ uma fibração de Hurewicz, então, $B$ será chamado espaço base, $E$ será chamado espaço total da fibração e, para cada $b \in B, F_{b}=p^{-1}(b)$ será chamado a fibra de $p$ sobre $b$. Embora $F_{b}$ possa variar para diferentes escolhas de $b \in B$, se $B$ for conexo por caminhos, a Propriedade do Levantamento de Homotopia restringe o tipo de homotopia de $F_{b}$, ou seja, se $B$ for conexo por caminhos, então todas as fibras $F_{b}=p^{-1}(b)$ são homotopicamente equivalentes ((DAVIS; KIRK, 2001), Theorem 6.12, pg. 117).

Definição 3.1.20. ((HATCHER, 2002), pg. 376) Uma função contínua $p: E \rightarrow B$ satisfazendo a Propriedade do Levantamento de Homotopia com respeito a $k$-discos $\mathbb{D}^{k}$, para todo $k \geq 0^{4}$, é chamada uma fibração de Serre $^{5}$.

Observação 3.1.21. Em relação ao conceito de fibrados localmente triviais e fibrações, são válidas as seguintes propriedades:

(a) Todo fibrado trivial e uma fibração de Hurewicz (vide (AGUILAR; GITLER; PRIETO, 2002), Lemma 4.5.4, pg. 126). E todo fibrado localmente trivial é uma fibração de Serre (vide (AGUILAR; GITLER; PRIETO, 2002), Theorem 4.5.6, pg. 127).

(b) Hatcher ((HATCHER, 2002), Proposition 4.48, pg. 379) mostrou que todo fibrado localmente trivial possui a propriedade do levantamento de homotopia com respeito a todos os pares $\mathrm{CW}(X, A)$. Além disso, a propriedade do levantamento de homotopia com respeito a todos os pares $\mathrm{CW}(X, A)$ é equivalente à propriedade do levantamento de homotopia para discos (equivalentemente para cubos).

4 Ou equivalentemente, com respeito a $n$ cubos $I^{n}=I \times \ldots \times I$, ou equivalentemente, CW complexos.

5 Em homenagem ao importante papel desempenhado por este conceito na tese de Jean-Pierre Serre. Este trabalho estabeleceu em Topologia Algébrica o uso das chamadas sequências espectrais e claramente separou as noções de fibrados e fibrações da noção de feixes (ambos estes conceitos tratados implicitamente no trabalho pioneiro de Jean Leray). 
(c) Desde que fibrados localmente triviais $p: E \rightarrow B$ possuem trivializações locais, ou seja, produtos cartesianos existem localmente sobre $B$, quando o espaço base $B$ for paracompacto, isto é suficiente para mostrar que todo fibrado localmente trivial é uma fibração de Hurewicz (vide (SPANIER, 1966), Section 2.7, Corollary 14, pg. 96).

A seguir, apresentamos a sequência exata longa dos grupos de homotopia associada a uma fibração.

Teorema 3.1.22. ((HOVEY, 1999), Lemma 2.4.16 pg. 55) Sejam $p: E \longrightarrow B$ uma fibração de Serre com fibra $F:=p^{-1}\left(b_{0}\right) \subseteq E$, para algum ponto base $b_{0}=p(x) \in B$ e $x \in F$. Então, existe uma sequência exata longa:

$$
\begin{aligned}
\cdots & \rightarrow \pi_{n}(F, x) \stackrel{i_{\star}}{\rightarrow} \pi_{n}(E, x) \stackrel{p_{\star}}{\rightarrow} \pi_{n}\left(B, b_{0}\right) \stackrel{\partial}{\rightarrow} \pi_{n-1}(F, x) \stackrel{i_{\star}}{\rightarrow} \pi_{n-1}(E, x) \rightarrow \cdots \\
& \rightarrow \pi_{1}(F, x) \stackrel{i_{\star}}{\rightarrow} \pi_{1}(E, x) \stackrel{p_{\star}}{\rightarrow} \pi_{1}\left(B, b_{0}\right) \stackrel{\partial}{\rightarrow} \pi_{0}(F, x) \stackrel{i_{\star}}{\rightarrow} \pi_{0}(E, x) \stackrel{p_{\star}}{\rightarrow} \pi_{0}\left(B, b_{0}\right)
\end{aligned}
$$

onde $\partial: \pi_{n}\left(B, b_{0}\right) \longrightarrow \pi_{n-1}(F, x)$ é um homomorfismo de grupos, quando $n>1$, chamado homomorfismo conectante. Essa sequência é natural com respeito a quadrados comutativos, conforme Figura 10:

Figura 10 - Naturalidade da sequência exata longa de uma fibração.

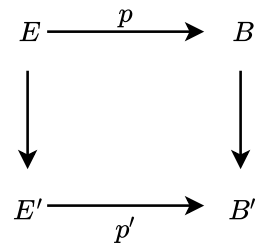

Fonte: Elaborada pelo autor.

onde $p$ e $p^{\prime}$ denotam fibrações de Serre.

Observação 3.1.23. No seguinte trecho da sequência exata obtida no Teorema 3.1.22:

$$
\cdots \rightarrow \pi_{1}\left(B, b_{0}\right) \stackrel{\partial}{\rightarrow} \pi_{0}(F, x) \stackrel{i_{\star}}{\rightarrow} \pi_{0}(E, x) \stackrel{p_{\star}}{\rightarrow} \pi_{0}\left(B, b_{0}\right)
$$

as aplicações envolvendo os conjuntos $\pi_{0}(F, x), \pi_{0}(E, x)$ e $\pi_{0}\left(B, b_{0}\right)$ não são, naturalmente, homomorfismos de grupos. Mas podemos pensar em definir um certo "núcleo" destas aplicações, considerando o "elemento neutro" como sendo a componente conexa contendo o ponto base em questão. Neste caso, a "exatidão" significa simplesmente que a imagem de uma aplicação é igual a este "núcleo" da aplicação subsequente. Assim, para este trecho da sequência estamos pensando em "exatidão" neste sentido.

Observação 3.1.24. Seja $X$ um espaço topológico. 
(i) Para $k \geq r \geq 1$, existe uma aplicação natural:

$$
\begin{aligned}
& \pi_{k, r}: \quad F(X, k) \quad \longrightarrow F(X, r) \\
& \left(x_{1}, \ldots, x_{r}, \ldots, x_{k}\right) \mapsto F\left(x_{1}, \ldots, x_{r}, \ldots, x_{k}\right):=\left(x_{1}, \ldots, x_{r}\right)
\end{aligned}
$$

obtida pela projeção sobre os primeiros r-fatores ((COHEN; PAKIANATHAN, ), Section 2, pg. 3).

(ii) Se $X$ é um $G$-espaço, então $G$ age pela ação diagonal sobre $F(X, k)$, como segue:

$$
\begin{aligned}
G \times F(X, k) & \longrightarrow F(X, k) \\
\left(g,\left(x_{1}, \ldots, x_{k}\right)\right) & \mapsto g\left(x_{1}, \ldots, x_{k}\right):=\left(g x_{1}, \ldots, g x_{k}\right),
\end{aligned}
$$

a qual é bem definida, pois, se $g x_{i}=g x_{j}$ então $x_{i}=g^{-1}\left(g x_{i}\right)=g^{-1}\left(g x_{j}\right)=x_{j}$.

(iii) $\pi_{k, r}: F(X, k) \longrightarrow F(X, r)$ é $G$-equivariante. De fato,

$$
\begin{aligned}
\pi_{k, r}\left(g\left(x_{1}, \ldots, x_{r}, \ldots, x_{k}\right)\right) & =\pi_{k, r}\left(g x_{1}, \ldots, g x_{r}, \ldots, g x_{k}\right) \\
& =\left(g x_{1}, \ldots, g x_{r}\right) \\
& =g\left(x_{1}, \ldots, x_{r}\right) \\
& =g \pi_{k, r}\left(x_{1}, \ldots, x_{r}, \ldots, x_{k}\right) .
\end{aligned}
$$

A aplicação $\pi_{k, r}: F(X, k) \longrightarrow F(X, r)$ com $k>r \geq 1$, dada na Observação 3.1.24 é muito útil para o estudo da natureza do espaço de configurações $F(X, k)$, especialmente quando $X$ é uma variedade sem bordo conexa, como mostra o resultado a seguir.

Teorema 3.1.25 (Fibração de Fadell e Neuwirth). ((COHEN; PAKIANATHAN, ), Theorem 2.5, pg. 3 e (FADELL; HUSSEINI, 2001), Theorem 1.1, pg. 6) Seja $M$ uma $m$-variedade topológica sem bordo conexa (não necessariamente compacta), onde $m \geq 2$. Então, a aplicação:

$$
\pi_{k, r}: F(M, k) \longrightarrow F(M, r), \quad k>r \geq 1
$$

é um fibrado localmente trivial, com fibra $F\left(M-Q_{r}, k-r\right)$.

Demonstração. Seja $x:=\left(x_{1}, \ldots, x_{r}\right) \in F(M, r)$ uma $r$-upla fixada. Como $M$ é uma $m$-variedade sem bordo, escolha vizinhanças de discos fechados mutuamente disjuntas $\bar{U}_{1}, \cdots, \bar{U}_{r}$ em $M$ tais que $x_{i} \in U_{i}=i n t \bar{U}_{i}, \forall 1 \leq i \leq r$. Para cada $i$, seja:

$$
\gamma_{i}^{*}: U_{i} \longrightarrow \operatorname{Top}_{s}(M)
$$

a aplicação obtida no Corolário 3.1.11, a qual denotaremos simplesmente por $\gamma_{i}$, satisfazendo:

(i) $\gamma_{i}(x)(x)=x_{i}, \quad \forall x \in U_{i}$. 
(ii) $\gamma_{i}(x)(y)=y, \quad \forall x \in U_{i}$ e $y \in M-U_{i}$.

Seja $Q_{r}^{x}=\left\{x_{1}, \ldots, x_{r}\right\}$. Podemos identificar a fibra de $\pi_{k, r}: F(M, k) \longrightarrow F(M, r)$ sobre o ponto $x=\left(x_{1}, \ldots, x_{r}\right)$, com o espaço $F\left(M-Q_{r}^{x}, k-r\right)$, pois

$$
\begin{aligned}
\pi_{k, r}^{-1}(x) & =\left\{\left(y_{1}, \ldots, y_{r}, \ldots, y_{k}\right) \in F(M, k) \mid \pi_{k, r}\left(y_{1}, \ldots, y_{r}, \ldots, y_{k}\right)=\left(x_{1}, \ldots, x_{r}\right)\right\} \\
& =\left\{\left(y_{1}, \ldots, y_{r}, \ldots, y_{k}\right) \in F(M, k) \mid \quad\left(y_{1}, \ldots, y_{r}\right)=\left(x_{1}, \ldots, x_{r}\right)\right\} \\
& \approx F\left(M-Q_{r}^{x}, k-r\right) \text { (homeomorfismo). }
\end{aligned}
$$

Agora, considerando $U:=U_{1} \times \cdots \times U_{r}$, definimos uma trivialização local $\psi_{U}$ :

Figura 11 - Quase trivialização local da Fibração de Fadell e Neuwirth.

$$
U \times F\left(M-Q_{r}^{x}, k-r\right) \stackrel{\psi_{U}}{\longrightarrow} \pi_{k, r}^{-1}(U)
$$
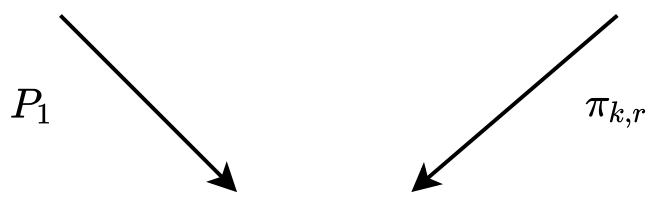

$U$

Fonte: Elaborada pelo autor.

Para cada $\left(x_{1}^{\prime}, \ldots, x_{r}^{\prime}\right) \in U$ e $y=\left(y_{1}, \ldots, y_{k-r}\right) \in F\left(M-Q_{r}^{x}, k-r\right)$, temos:

$$
\begin{aligned}
\psi_{U}\left(\left(x_{1}^{\prime}, \ldots, x_{r}^{\prime}\right), y\right): & =\left(x_{1}^{\prime}, \ldots, x_{r}^{\prime},(\underbrace{\left(\gamma_{1}\left(x_{1}^{\prime}\right)\right)^{-1} \circ \cdots \circ\left(\gamma_{r}\left(x_{r}^{\prime}\right)\right)^{-1}}_{=\Delta \in T o p_{s}(M)})(y)\right) \\
& =\left(x_{1}^{\prime}, \ldots, x_{r}^{\prime}, \Delta\left(y_{1}\right), \ldots, \Delta\left(y_{k-r}\right)\right) .
\end{aligned}
$$

Como $y=\left(y_{1}, \ldots, y_{k-r}\right) \in F\left(M-Q_{r}^{x}, k-r\right), y_{j} \neq x_{i}, \forall j=1, \ldots, k-r$ e $\forall i=1, \ldots, r$ e

$$
\begin{gathered}
\Delta\left(x_{i}\right)=\left(\left(\gamma_{1}\left(x_{1}^{\prime}\right)\right)^{-1} \circ \cdots \circ\left(\gamma_{r}\left(x_{r}^{\prime}\right)\right)^{-1}\right)\left(x_{i}\right)=x_{i}^{\prime} \text { para todo } 1 \leq i \leq r, \text { pois : } \\
\left(\gamma_{r}\left(x_{r}^{\prime}\right)\right)^{-1}\left(x_{i}\right)= \begin{cases}x_{i}^{\prime}, & \text { se } i=r, \\
x_{i}, & \text { se } i \neq r,\end{cases}
\end{gathered}
$$

concluímos que $\Delta\left(y_{j}\right) \neq x_{i}^{\prime}, \forall j=1, \ldots, k-r$ e $\forall i=1, \ldots, r$. Logo,

$$
\psi_{U}\left(\left(x_{1}^{\prime}, \ldots, x_{r}^{\prime}\right), y\right) \in \pi_{k, r}^{-1}(U) \subset F(M, k)
$$

e, assim, $\psi_{U}$ é bem definida e é contínua.

Observemos que a fibra $F\left(M-Q_{r}^{x}, k-r\right)$ depende da escolha do ponto $x=\left(x_{1}, \ldots, x_{r}\right)$. Por outro lado, como toda $m$-variedade conexa $M$, com $m \geq 2$, é $r$-homogênea, $\forall r \geq 1$, 
existe $h_{x} \in \operatorname{Top}_{s}(M)$ tal que $h_{x}\left(q_{i}\right)=x_{i}, \forall 1 \leq i \leq r$ (vide Teorema B.2.9), onde $q=\left(q_{1}, \ldots, q_{r}\right)$ é o ponto base escolhido em $F(M, r)$. Assim, $h_{x}$ induz um homeomorfismo:

$$
h_{x}^{\prime}: F\left(M-Q_{r}, k-r\right) \longrightarrow F\left(M-Q_{r}^{x}, k-r\right), \text { onde } Q_{r}=\left\{q_{1}, \ldots, q_{r}\right\} .
$$

Agora, definimos $\varphi_{U}:=\psi_{U} \circ\left(I d_{U} \times h_{x}^{\prime}\right)$. Então, $\varphi_{U}$ é uma trivialização local sobre $U$ :

Figura 12 - Trivialização local da Fibração de Fadell e Neuwirth.

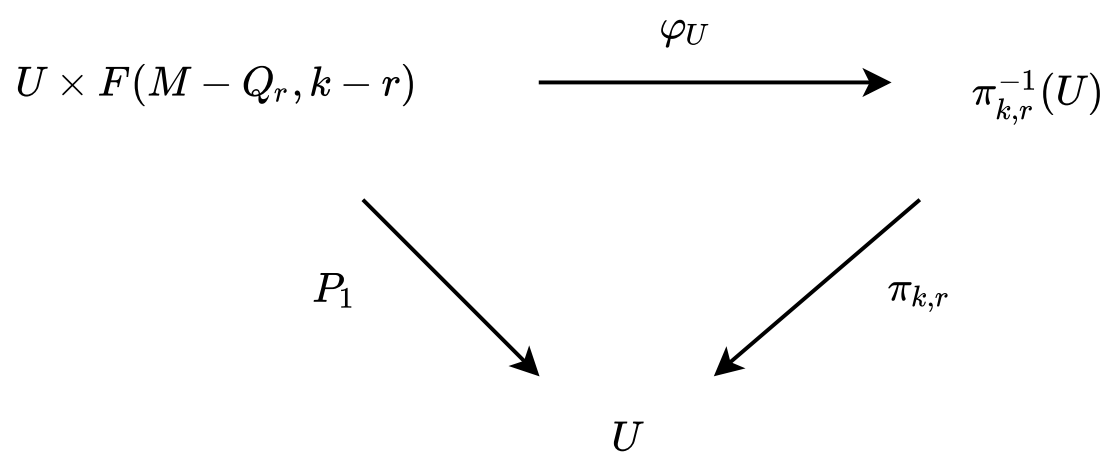

Fonte: Elaborada pelo autor.

onde a fibra agora é fixada, ou seja, independente do ponto $x$.

Observação 3.1.26. Observemos que se $M$ é uma variedade topológica conexa (sem bordo) então, segue da Proposição 2.1.19, que o espaço de configurações $F(M, k)$ é uma variedade topológica, logo é um espaço paracompacto, pelo Teorema 1.4.8. Portanto, o fibrado localmente trivial $\pi_{k, r}: F(M, k) \longrightarrow F(M, r)$ obtido no Teorema 3.1.25 é uma fibração de Hurewicz (vide Observação 3.1.21-(c)).

O próximo resultado fornece condições que garantem quando um espaço de configurações é conexo por caminhos. A sua demonstração seguirá do Teorema 3.1.25.

Corolário 3.1.27. ((COHEN; PAKIANATHAN, ), Lemma 2.8, pg. 4). Seja $M$ uma $m$ variedade topológica conexa (sem bordo) tal que $M$ permanece conexa quando é perfurada em $k-1 \geq 0$ pontos. Então, $F(M, k)$ é conexo por caminhos (o que implicará que $S F(M, k)$ também é conexo por caminhos).

Demonstração. A prova será feita por indução sobre $k$. Quando $k=1$, sabemos que $F(M, 1)=M$, e o resultado segue. Suponhamos que $F(M, l)$ seja conexo por caminhos, para todo $1 \leq l \leq k-1$ e para qualquer variedade conexa (sem bordo) $M$ tal que $M$ permanece conexa quando é perfurada em $l-1 \geq 0$ pontos. Então, pelo Teorema 3.1.25,

$$
\pi_{k, 1}: F(M, k) \longrightarrow F(M, 1)
$$

é um fibrado localmente trivial (logo uma fibração, pela Observação 3.1.26) com fibra $F\left(M-Q_{1}, k-1\right)$. Note ainda que $M-Q_{1}$ é uma variedade conexa (sem bordo) tal que 
$M-Q_{1}$ ainda permanece conexa quando é perfurada em $k-2 \geq 0$ pontos. Assim, pela hipótese de indução, o espaço base e a fibra da fibração (3.1.2) são ambos conexos por caminhos e, portanto, o espaço total $F(M, k)$ da fibração (3.1.2) também é conexo por caminhos. Este último fato, segue olhando para o último trecho da sequência exata longa de homotopia associada a uma fibração (vide Teorema 3.1.22 e Observação 3.1.23):

$$
\{\text { ponto }\}=\pi_{0}\left(F\left(M-Q_{1}, k-1\right), x\right) \stackrel{i_{\star}}{\rightarrow} \pi_{0}(F(M, k), x) \stackrel{p_{\star}}{\rightarrow} \pi_{0}\left(M, b_{0}\right)=\{\text { ponto }\} .
$$

Pela "exatidão" em $\pi_{0}(F(M, k), x)$, obtemos:

$$
\begin{aligned}
\pi_{0}(F(M, k), x) & =\operatorname{ker}\left(p_{\star}\right) \\
& =\operatorname{Im}\left(i_{\star}\right) \\
& =\{\text { ponto } .
\end{aligned}
$$

Assim, $F(M, k)$ é conexo por caminhos, para todo $k \geq 1$.

Observação 3.1.28. Segue do Corolário 3.1.27, que se $M$ for uma variedade conexa (sem bordo) de dimensão pelo menos 2, então todos seus espaços de configurações serão conexos por caminhos (vide Proposição 2.1.24).

Corolário 3.1.29. Seja $M$ uma variedade $r-$ conexa $^{6}$ sem bordo tal que $M$ permanece $r$-conexa quando é perfurada em $k-1 \geq 0$ pontos. Então, $F(M, k)$ é $r$-conexo.

Demonstração. A prova será feita por indução sobre $k$. Quando $k=1$, o resultado segue da hipótese. Suponhamos que $F(M, l)$ seja $r$-conexo, pata todo $1 \leq l \leq k-1$ e para toda variedade (sem bordo) $r$-conexa $M$, tal que $M$ permanece $r$-conexa quando é perfurada em $l-1 \geq 0$ pontos. Então, pelo Teorema 3.1.25,

$$
\pi_{k, 1}: F(M, k) \longrightarrow F(M, 1)
$$

é uma fibração com fibra $F\left(M-Q_{1}, k-1\right)$, com $M-Q_{1}$ variedade (sem bordo) $r$-conexa tal que $M-Q_{1}$ permanece $r$-conexa quando é perfurada em $k-2 \geq 0$ pontos. Logo, usando a sequência exata longa associada à fibração (3.1.3) (vide Teorema 3.1.22), temos:

$$
\cdots \longrightarrow \pi_{q}\left(F\left(M-Q_{1}, k-1\right)\right) \longrightarrow \pi_{q}(F(M, k)) \longrightarrow \pi_{q}(F(M, 1)) \longrightarrow \cdots .
$$

Assim, segue por indução que o espaço base e a fibra são ambos $r$-conexos e, portanto, o espaço total $F(M, k)$ da fibração (3.1.3) também é $r$-conexo.

Exemplo 3.1.30. Lembremos que, quando $\mathbb{R}^{n}$ é perfurado em $(k-1)$ pontos, obtemos um espaço que tem o mesmo tipo de homotopia de um buquê de $(k-1)$ cópias de $(n-1)$-esferas:

$$
\mathbb{R}^{n}-Q_{k-1} \simeq \bigvee^{k-1} \mathbb{S}^{n-1}, \quad Q_{k-1}=\left\{q_{1}, \ldots, q_{k-1}\right\}, \quad \text { (vide Corolário B.4.2). }
$$

6 Vide Capítulo 1, Definição 1.7.5 e Observação 1.7.6. 
Mas, se $n \geq 2$, segue de ((FUCHS; VIRO, 2003), Corollary F, pg. 86), que:

$\pi_{i}\left(\bigvee^{k-1} \mathbb{S}^{n-1}\right)$ é trivial, $\forall i<n-1$ e $\pi_{n-1}\left(\bigvee^{k-1} \mathbb{S}^{n-1}\right)$ é um grupo abeliano livre,

tendo como base as classes de homotopia de inclusões

$$
S_{\alpha}^{n-1} \rightarrow \bigvee_{\alpha} S_{\alpha}^{n-1}
$$

Assim, $\mathbb{R}^{n}-Q_{k-1}$ é $(n-2)$ - conexo, para todo $n \geq 2$. Portanto, pelo Corolário 3.1.29, $F\left(\mathbb{R}^{n}, k\right)$ é $(n-2)$-conexo, para todo $n \geq 2$ e para todo $k \geq 1$. Em particular, $F\left(\mathbb{R}^{n}, k\right)$ é simplesmente conexo, $\forall n \geq 3$.

Exemplo 3.1.31. Observemos que $\mathbb{S}^{n}-Q_{k-1}$ é homeomorfa a $\mathbb{R}^{n}-Q_{k-2}$, onde $Q_{k}$ denota um subconjunto com $k$-elementos. Assim, quando $\mathbb{S}^{n}$ é perfurada em $(k-1)$ pontos, obtemos um espaço que tem o mesmo tipo de homotopia de um buquê de $(k-2)$ cópias de $(n-1)$-esferas:

$$
\mathbb{S}^{n}-Q_{k-1} \approx \mathbb{R}^{n}-Q_{k-2} \simeq \bigvee^{k-2} \mathbb{S}^{n-1},
$$

(vide Corolário B.4.2). Logo, usando os mesmos argumento do Exemplo 3.1.30, temos que $\mathbb{S}^{n}-Q_{k-1}$ é $(n-2)$-conexa, se $n \geq 2$. Portanto, pelo Corolário 3.1.29, segue que $F\left(\mathbb{S}^{n}, k\right)$ é $(n-2)$-conexo, $\forall n \geq 2, \quad \forall k \geq 1$. Em particular, $F\left(\mathbb{S}^{n}, k\right)$ é simplesmente conexo, $\forall n \geq 3$.

Exemplo 3.1.32. O espaço projetivo complexo $\mathbb{C P}^{n}$ é 1 -conexo, para todo $n$ (vide (MCCLEARY, 2001), Part II, Section 5, pag. 144). Além disso, $\mathbb{C P}^{n}-\{p t o\}$ tem mesmo tipo de homotopia $\mathbb{C P}^{n-1}$, o qual é 1 -conexo. Assim, segue do Corolário 3.1.29 que o espaço de configurações $F\left(\mathbb{C P}^{n}, 2\right)$ é 1-conexo.

No caso de variedades com buracos, obtemos o seguinte resultado.

Corolário 3.1.33. ((FADELL; NEUWIRTH, 1962), Theorem 3, pg. 114) Seja $M$ uma variedade topológica (sem bordo) conexa (não necessariamente compacta), com $\operatorname{dim} M \geq 2$. Então, a aplicação

$$
\pi_{k, r}: F\left(M-Q_{m}, k\right) \longrightarrow F\left(M-Q_{m}, r\right)
$$

é um fibrado localmente trivial, com fibra $F\left(M-Q_{m+r}, k-r\right)$, com $m \geq 0$ e $k \geq r$.

Demonstração. Pela Proposição 2.1.24, $M-Q_{m}$ é uma variedade topológica conexa de dimensão maior ou igual a 2. Assim, o resultado segue do Teorema 3.1.25.

Definição 3.1.34. Sejam $\pi$ um grupo e $X$ um espaço topológico conexo por caminhos satisfazendo as seguintes condições, para algum natural $n$ fixado:

$$
\pi_{i}(X)=0, \quad \forall i \neq n, \quad \text { e } \pi_{n}(X) \cong \pi
$$

Então, $X$ é chamado espaço de Eilenberg-MacLane ou simplesmente um $K(\pi, n)$-espaço (vide (HATCHER, 2002), Chapter 4, Section 4.2, pg. 365). 
Exemplo 3.1.35. ((HATCHER, 2002), Example 1B.1, pg. 88) $\mathbb{S}^{1}$ é um $K(\mathbb{Z}, 1)$-espaço. Mais geralmente, um grafo conexo é um $K(\pi, 1)$ - espaço, com $\pi$ um grupo livre. $\mathbb{R}^{m}$ é um $K(0, n)$-espaço, $\forall n \geq 1$.

Observação 3.1.36. Observemos que a 2-esfera $\mathbb{S}^{2}$ e o plano projetivo $\mathbb{R P}^{2}$ não são espaços do tipo $K(\pi, 1)$, pois $\pi_{2}\left(\mathbb{R P}^{2}\right) \cong \pi_{2}\left(\mathbb{S}^{2}\right) \cong \mathbb{Z}$. Isso segue da sequência exata longa de homotopia associada ao recobrimento a duas folhas $\mathbb{Z}_{2} \hookrightarrow \mathbb{S}^{2} \longrightarrow \mathbb{R P}^{2}$ e de (HATCHER, 2002), Corollary 4.25, pg. 361.

Observação 3.1.37. Recordemos que, para qualquer superfície fechada $M$, orientável ou não, diferente da 2-esfera $\mathbb{S}^{2}$ e do plano projetivo $\mathbb{R P}^{2}$, existe um recobrimento universal $\mathbb{R}^{2} \longrightarrow M$. Como $\mathbb{R}^{2}$ é contrátil, todos os grupos de homotopia superiores de $M$ são triviais. Assim, $M$ é um $K(\pi, 1)$-espaço ((HATCHER, 2002), Example 1B.2, pg. 88). Além disso, para qualquer superfície fechada $M$, orientável ou não, diferente de $\mathbb{S}^{2}$ e do plano projetivo $\mathbb{R P}^{2}$, com $m \geq 1$ buracos, tem o mesmo tipo de homotopia de um buquê de 1-esferas. Por outro lado, a 2-esfera $\mathbb{S}^{2}$ com $m \geq 2$ buracos também possui o mesmo tipo de homotopia de um buquê de 1-esferas. Assim, o grupo fundamental de tais espaços é um grupo livre e os grupos de homotopia superiores são triviais (vide (FUCHS; VIRO, 2003), 11.4, pg. 84). As únicas superfícies simplesmente conexas sem bordo são $\mathbb{S}^{2}$ e $\mathbb{R}^{2}$. Superfícies não fechadas retraem-se por deformação sobre grafos, assim tais superfícies são $K(\pi, 1)$-espaços, com $\pi$ um grupo livre.

Uma outra aplicação do Teorema 3.1.25 é o resultado a seguir, o qual descreve os espaços de configurações para variedades topológicas fechadas conexas de dimensão 2 como $K(\pi, 1)$-espaços (vide (COHEN; PAKIANATHAN, ), Theorem 2.7, pg. 4 e (FADELL; NEUWIRTH, 1962), Section II, Corollary 2.2, pg. 113).

Teorema 3.1.38. Suponhamos que $M=\mathbb{R}^{2}$ ou que $M$ seja uma superfície fechada (compacta, conexa e sem bordo), orientável ou não.

(1) Então, $F\left(M-Q_{m}, k\right)$ é um $K(\pi, 1)$-espaço, o que implicará que $S F\left(M-Q_{m}, k\right)$ também é um $K(\pi, 1)$-espaço, para todo $m \geq 1$ e para todo $k \geq 1$.

(2) Se $M$ é diferente de $\mathbb{S}^{2}$ e do plano projetivo $\mathbb{R P}^{2}$, então $F(M, k)$ e $S F(M, k)$ são $K(\pi, 1)$-espaços, para todo $k \geq 1$.

Demonstração. A prova será feita por indução sobre $k$.

Consideremos, primeiramente, o caso $k=1$. Observemos que:

(a) $\mathbb{R}^{2}-Q_{m}$ tem o mesmo tipo de homotopia de um buquê de 1-esferas, $\forall m \geq 1$ e, portanto, $\mathbb{R}^{2}-Q_{m}=F\left(\mathbb{R}^{2}-Q_{m}, 1\right)$ é um $K(\pi, 1)$-espaço, para todo $m \geq 1$. 
(b) Sabemos que $S^{2}-Q_{1}$ é homeomorfa a $\mathbb{R}^{2}$. Além disso, se $m \geq 2$, $\mathbb{S}^{2}-Q_{m}$ tem o mesmo tipo de homotopia de um buquê de 1-esferas. Portanto, segue do Exemplo 3.1.35 e da Observação 3.1.37 que $\mathbb{S}^{2}-Q_{m}=F\left(\mathbb{S}^{2}-Q_{m}, 1\right)$ é um $K(\pi, 1)$-espaço, para todo $m \geq 1$.

(c) Um espaço de recobrimento a duas folhas para $\mathbb{R} \mathbb{P}^{2}-Q_{m}$, com $m \geq 1$, é $\mathbb{S}^{2}-Q_{2 m}$, que possui o mesmo tipo de homotopia de um buquê de 1-esferas. Portanto, usando a sequência exata longa de homotopia associada a este recobrimento concluímos que $\mathbb{R P}^{2}-Q_{m}=F\left(\mathbb{R P}^{2}-Q_{m}, 1\right)$ é um $K(\pi, 1)$-espaço, para todo $m \geq 1$.

(d) Se $M$ é diferente de $\mathbb{S}^{2}$ e do plano projetivo $\mathbb{R P}^{2}$ e se $m \geq 1$, então $M-Q_{m}$ tem o mesmo tipo de homotopia de um buquê de 1-esferas, logo $M-Q_{m}=F\left(M-Q_{m}, 1\right)$ é um $K(\pi, 1)$-espaço, pela Observação 3.1.37, onde é um $\pi$ é um grupo livre de posto finito.

(e) $\mathbb{R}^{2}$ é um $K(\pi, 1)$-espaço e, portanto, $\mathbb{R}^{2}=F\left(\mathbb{R}^{2}, 1\right)$ é um $K(\pi, 1)$-espaço.

(f) Para qualquer superfície fechada $M$ diferente de $\mathbb{S}^{2}$ e de $\mathbb{R P}^{2}$, temos da Observação 3.1.37 que $M=F(M, 1)$ é um $K(\pi, 1)$-espaço.

Suponhamos agora $k>1$ e que o resultado seja válido para todos os valores $1 \leq l \leq k-1$. Pelo Teorema 3.1.25, aplicado à variedade conexa sem bordo $M-Q_{m}$, a aplicação

$$
\pi_{k, 1}: F\left(M-Q_{m}, k\right) \longrightarrow F\left(M-Q_{m}, 1\right)
$$

é uma fibração com fibra $F\left(M-Q_{m+1}, k-1\right)$. Deste modo, pela hipótese de indução, o espaço base e a fibra desta fibração são $K(\pi, 1)$-espaços e, pela sequência exata longa dos grupos de homotopia de uma fibração, concluímos que o espaço total é um $K(\pi, 1)$-espaço. De fato, para $q \geq 2$ obtemos:

$$
0=\pi_{q}\left(F\left(M-Q_{m+1}, k-1\right)\right) \stackrel{i_{\star}}{\rightarrow} \pi_{q}\left(F\left(M-Q_{m}, k\right)\right) \stackrel{p_{\star}}{\rightarrow} \pi_{q}\left(F\left(M-Q_{m}, 1\right)\right)=0
$$

e pela exatidão em $\pi_{q}\left(F\left(M-Q_{m}, k\right)\right)$, segue

$$
\pi_{q}\left(F\left(M-Q_{m}, k\right)\right)=0 .
$$

Além disso, $F\left(M-Q_{m}, k\right)$ é conexo por caminhos (vide Observação 3.1.28). Assim, o espaço total $F\left(M-Q_{m}, k\right)$ é um $K(\pi, 1)$-espaço.

Exemplo 3.1.39. Considere as superfícies fechadas (compactas, conexas e sem bordo) e orientáveis: o toro $T=\mathbb{S}^{1} \times \mathbb{S}^{1}$ e o toro duplo $T \# T$. Pelo Teorema 3.1.38, seus espaços de configurações $F(T, k), S F(T, k)$ e $F(T \# T, k), S F(T \# T, k)$ são $K(\pi, 1)$-espaços, $\forall k \geq 1$. 
Exemplo 3.1.40. A garrafa de Klein $\mathbb{K}$ é uma superfície fechada não-orientável. Assim, pelo Teorema 3.1.38, seus espaços de configurações $F(\mathbb{K}, k)$ e $S F(\mathbb{K}, k)$ são $K(\pi, 1)$-espaços, $\forall k \geq 1$.

Observação 3.1.41. Segue do Teorema 3.1.38, que se $M=\mathbb{R}^{2}$ ou $M$ é qualquer superfície fechada, exceto a 2-esfera $\mathbb{S}^{2}$ e o plano projetivo $\mathbb{R P}^{2}$, o tipo de homotopia de $F(M, k)$ e $S F(M, k)$ é completamente determinado por seu grupo fundamental ((HATCHER, 2002), Theorem 1B.8, pg. 90). Por essa razão, um interessante problema em Topologia Algébrica consiste em se calcular tais grupos de homotopia, os quais são definidos como grupos de tranças de superfícies ((COHEN; PAKIANATHAN, ), Section 2, pg. 4).

Teorema 3.1.42. ((BIRMAN, 1969), Theorem 1, pg.42) Seja $M$ uma variedade suave fechada (compacta, conexa e sem bordo). Então, para cada $s \geq 1$ a aplicação inclusão

$$
i: F(M, k) \hookrightarrow \prod_{j=1}^{k} M
$$

induz um homomorfismo

$$
i_{*}: \pi_{s}(F(M, k)) \longrightarrow \prod_{j=1}^{k} \pi_{s}(M),
$$

o qual é sobrejetor, se $\operatorname{dim}(M)>s$ e também injetor, se $\operatorname{dim}(M)>s+1$.

No caso particular $s=1$, temos o seguinte

Corolário 3.1.43. ((COHEN, 2010), Remark 8.2, pg. 200) Para uma variedade $M$ suave fechada, a inclusão

$$
i: F(M, k) \hookrightarrow \prod_{j=1}^{k} M
$$

induz um homomorfismo

$$
i_{*}: \pi_{1}(F(M, k)) \longrightarrow \prod_{i=1}^{k} \pi_{1}(M),
$$

o qual é isomorfismo, se $\operatorname{dim}(M) \geq 3$ e sobrejetor, se $\operatorname{dim}(M)=2$.

Corolário 3.1.44. Seja $M$ uma variedade suave fechada, com $\operatorname{dim}(M) \geq 3$. Então:

$F(M, k)$ é simplesmente conexa se, e somente se, $M$ é simplesmente conexa.

\subsection{Grupos de Tranças e Espaços de Configurações}

Nesta seção, definiremos o conceito de grupos de tranças para uma variedade, em termos do grupo fundamental de seus espaços de configurações. 
Definição 3.2.1. ((BIRMAN, 1974)) Seja $M$ uma variedade topológica de dimensão $\operatorname{dim} M \geq 2$. Para todo $k \geq 1$, definimos o grupo de tranças puras em $k$-cordas sobre $M$, denotado por $P B_{k}(M)$ como sendo o grupo fundamental do espaço de configurações $F(M, k)$. Analogamente, definimos o grupo de traças em $k$-cordas sobre $M$, denotado por $B_{k}(M)$, como sendo o grupo fundamental do espaço de configurações $S F(M, k)$, ou seja:

$$
P B_{k}(M):=\pi_{1}\left(F(M, k), x_{0}\right) \text { e } B_{k}(M):=\pi_{1}\left(S F(M, k), y_{0}\right), \quad \forall k \geq 1 .
$$

Observação 3.2.2. Quando $M$ for o 2-dimensional espaço Euclideano $\mathbb{R}^{2}$ usaremos simplesmente as notações:

$$
B_{k}\left(\mathbb{R}^{2}\right)=B_{k} \text { e } P B_{k}\left(\mathbb{R}^{2}\right)=P B_{k}, \quad \forall k \geq 1,
$$

os quais são conhecidos como o grupo de tranças de Artin e o grupo de tranças puras de Artin, respectivamente (vide (COHEN; PAKIANATHAN, ), Definition 3.6, pg. 7)

Observação 3.2.3. Segue do Teorema 3.1.38 e da Definição 3.2.1, que os espaços de configurações $F\left(\mathbb{R}^{2}, k\right)$ e $S F\left(\mathbb{R}^{2}, k\right)$ são espaços do tipo $K(\pi, 1)$, para todo $k \geq 1$, onde:

$$
F\left(\mathbb{R}^{2}, k\right)=K\left(P B_{k}, 1\right) \text { e } S F\left(\mathbb{R}^{2}, k\right)=K\left(B_{k}, 1\right), \quad \forall k \geq 1
$$

(Vide (COHEN; PAKIANATHAN, ), Section 3, pg. 7)

Observação 3.2.4. Note que, pelo Corolário 3.1.43, para uma variedade suave fechada $M$, com $\operatorname{dim} M \geq 3$, temos:

$$
P B_{k}(M):=\pi_{1}(F(M, k)) \cong \prod_{i=1}^{k} \pi_{1}(M), \quad \forall k \geq 1,
$$

ou seja, o grupo de tranças puras $P B_{k}(M)$ é determinado pelo grupo fundamental $\pi_{1}(M)$. Em particular, se $M$ for simplesmente conexa, segue do Corolário 3.1.44 que $F(M, k)$ será simplesmente conexo, ou seja,

$$
P B_{k}(M):=\pi_{1}(F(M, k))=1, \quad \forall k \geq 1 .
$$

Consequentemente, do Teorema B.3.2, concluímos que:

$$
B_{k}(M):=\pi_{1}(S F(M, k))=\pi_{1}\left(F(M, k) / S_{k}\right) \cong S_{k}, \quad \forall k \geq 1 .
$$

(Vide (FADELL; NEUWIRTH, 1962), Theorem 9, pg. 118)). Por essa razão, o estudo dos grupos de tranças possui relevância no caso de variedades de dimensão igual a 2 , as chamadas superfícies.

Exemplo 3.2.5. Segue do Exemplo 3.1.31, que $F\left(\mathbb{R}^{n}, k\right)$ é simplesmente conexo, $\forall n \geq 3$. Logo, pela Observação 3.2.4, os grupos de tranças para o $n$-dimensional espaço Euclideano $\mathbb{R}^{n}$, com $n \geq 3$ são:

$$
P B_{k}\left(\mathbb{R}^{n}\right):=\pi_{1}\left(F\left(\mathbb{R}^{n}, k\right)\right)=1 \quad \text { e } \quad B_{k}\left(\mathbb{R}^{n}\right)=\pi_{1}\left(S F\left(\mathbb{R}^{n}, k\right)\right) \cong S_{k}, \quad \forall k \geq 1 .
$$


Exemplo 3.2.6. Segue do Exemplo 3.1 .30 que $F\left(\mathbb{S}^{n}, k\right)$ é simplesmente conexo, $\forall n \geq 3$. Assim, da Observação 3.2.4, os grupos de tranças para a $n$-esfera, $\mathbb{S}^{n}$, com $n \geq 3$ são: $P B_{k}\left(\mathbb{S}^{n}\right):=\pi_{1}\left(F\left(\mathbb{S}^{n}, k\right)\right)=1 \quad$ e $\quad B_{k}\left(\mathbb{S}^{n}\right)=\pi_{1}\left(S F\left(\mathbb{S}^{n}, k\right)\right) \cong S_{k}, \quad \forall k \geq 1$ 

CAPÍTULO

\section{4}

SEÇÕES DA FIBRAÇÃO DE FADELL E

NEUWIRTH

\subsection{Os grupos de homotopia de $F\left(M \times \mathbb{R}^{n}, k\right)$}

Neste capítulo, nosso principal objetivo é obter os grupos de homotopia dos espaços de configurações $F\left(M \times \mathbb{R}^{n}, k\right)$ ), para todo $k \geq 1$, no qual $M$ é uma variedade topológica conexa.

Definição 4.1.1. ((ROBINSON, 2003), pg. 75) Sejam $H$ e $N$ dois grupos e consideremos:

$$
\begin{aligned}
\varphi: H & \longrightarrow \operatorname{Aut}(N) \\
h & \mapsto \varphi_{h}: N \rightarrow N
\end{aligned}
$$

um homomorfismo do grupo $H$ ao grupo dos automorfismos de $N$. O produto semi-direto (externo) de $H$ e $N$, com respeito ao homomorfismo $\varphi$, o qual será denotado por $N \rtimes_{\varphi} H$, é o conjunto $N \times H$, equipado com a seguinte operação:

$$
\begin{aligned}
\cdot:(N \times H) \times(N \times H) & \longrightarrow(N \times H) \\
\left(\left(n_{1}, h_{1}\right),\left(n_{2}, h_{2}\right)\right) & \mapsto\left(n_{1}, h_{1}\right) \cdot\left(n_{2}, h_{2}\right):=\left(n_{1} \varphi_{h_{1}}\left(n_{2}\right), h_{1} h_{2}\right) .
\end{aligned}
$$

Observação 4.1.2. Mostra-se que o produto semi-direto $N \rtimes_{\varphi} H$ é um grupo, com elemento identidade $\left(1_{N}, 1_{H}\right) \in N \times H$ e, para cada $(n, h) \in N \rtimes_{\varphi} H$, seu inverso é dado por $\left(\varphi_{h^{-1}}\left(n^{-1}\right), h^{-1}\right)$ (vide (ROBINSON, 2003), pg. 75 ).

Observação 4.1.3. Suponhamos que $\varphi: H \longrightarrow A u t(N)$ seja o homomorfismo trivial $h \mapsto \varphi_{h}=I d_{N}$, para todo $h \in H$. Então:

$$
\left(n_{1}, h_{1}\right) \cdot\left(n_{2}, h_{2}\right):=\left(n_{1} \varphi_{h_{1}}\left(n_{2}\right), h_{1} h_{2}\right)=\left(n_{1} n_{2}, h_{1} h_{2}\right) .
$$

Neste caso, o produto semi-direto coincide com o produto direto:

$$
N \rtimes_{\varphi} H=N \oplus H=N \times H .
$$


Observação 4.1.4. Note que, se $A u t(N)=\left\{I d_{N}\right\}$, então

$$
N \rtimes_{\varphi} H=N \oplus H=N \times H .
$$

Definição 4.1.5. Uma sequência exata curta de grupos e homomorfismos de grupos

$$
1 \longrightarrow N \stackrel{\beta}{\longrightarrow} G \stackrel{\alpha}{\longrightarrow} H \longrightarrow 1
$$

cinde, se existe um homomorfismo de grupos $\gamma: H \longrightarrow G$ tal que $\alpha \circ \gamma=I d_{H}$, ou seja, $\alpha$ possui inversa à direita.

Exemplo 4.1.6. O grupo $G$ definido pelo produto semi-direto de $H$ e $N$, ou seja, $G=N \rtimes_{\varphi} H$, se encaixa em uma sequência exata curta que cinde:

$$
1 \longrightarrow N \stackrel{i_{1}}{\longrightarrow} G \underset{i_{2}}{\stackrel{\pi}{\rightleftarrows}} H \longrightarrow 1
$$

na qual $i_{1}(n)=\left(n, 1_{H}\right), \quad \pi(n, h)=h$ e $i_{2}(h)=\left(1_{N}, h\right), \forall n \in N$ e $\forall h \in H$. Além disso,

$$
\left(\pi \circ i_{2}\right)(h)=\pi\left(1_{N}, h\right)=h=I d_{H}(h), \quad \forall h \in H .
$$

Lema 4.1.7. Dada uma sequência exata curta de grupos e homomorfismos de grupos

$$
1 \longrightarrow N \stackrel{\beta}{\longrightarrow} G \stackrel{\alpha}{\longrightarrow} H \longrightarrow 1
$$

as seguintes condições são equivalentes:

(i) A sequência cinde.

(ii) Existe um homomorfismo $\varphi: H \longrightarrow A u t(N)$ e um isomorfismo $\Omega: N \rtimes_{\varphi} H \longrightarrow G$ que torna o seguinte diagrama comutativo (vide Figura 13):

Figura 13 - Isomorfismo $\Omega$

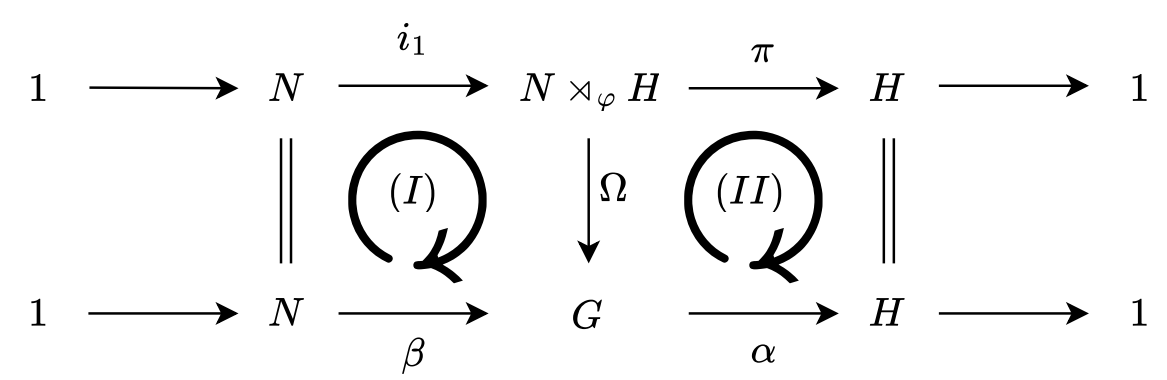

Fonte: Elaborada pelo autor. 
no qual, a sequência ( $\star$ é a sequência exata curta dada no Exemplo 4.1.6, a qual cinde.

Demonstração. Vejamos que $(i) \Longrightarrow(i i)$. Seja $\gamma: H \longrightarrow G$ tal que $\alpha \circ \gamma=i d_{H}$. Definimos

$$
\varphi: H \longrightarrow \operatorname{Aut}(N), \varphi(h):=\varphi_{h},
$$

onde $\varphi_{h}: N \longrightarrow N$ é definida por

$$
\varphi_{h}(n)=\beta^{-1}\left(\gamma(h) \beta(n) \gamma\left(h^{-1}\right)\right), \quad \forall n \in N
$$

Note que, $\gamma(h) \beta(n) \gamma\left(h^{-1}\right) \in \operatorname{Ker}(\alpha)=\operatorname{Im}(\beta)$, pois

$$
\alpha\left(\gamma(h) \beta(n) \gamma\left(h^{-1}\right)\right)=\underbrace{\alpha(\gamma(h))}_{h} \underbrace{\alpha(\beta(n))}_{1_{H}} \underbrace{\alpha\left(\gamma\left(h^{-1}\right)\right)}_{h^{-1}}=h \cdot 1_{H} \cdot h^{-1}=1_{H},
$$

e $\left.\beta\right|_{\operatorname{Im}(\beta)}$ é um isomorfismo. Assim, $\varphi_{h}$ é um automorfismo com inversa $\varphi_{h^{-1}}$. Além disso, $\varphi$ é um homomorfismo de grupos.

Vejamos que existe um isomorfismo $\Omega: N \rtimes_{\varphi} H \longrightarrow G$. Definimos $\Omega: N \rtimes_{\varphi} H \longrightarrow G$ por:

$$
\Omega(n, h)=\beta(n) \gamma(h)
$$

$\Omega$ é um homomorfismo de grupos, pois:

$$
\begin{aligned}
\Omega\left(\left(n_{1}, h_{1}\right) \cdot\left(n_{2}, h_{2}\right)\right) & =\Omega\left(n_{1} \varphi_{h_{1}}\left(n_{2}\right), h_{1} h_{2}\right) \\
& =\beta\left(n_{1} \varphi\left(h_{1}\right)\left(n_{2}\right)\right) \gamma\left(h_{1} h_{2}\right) \\
& =\beta\left(n_{1}\right) \underbrace{\beta\left(\varphi\left(h_{1}\right)\left(n_{2}\right)\right)}_{\operatorname{por}(4.1 .3)} \gamma\left(h_{1}\right) \gamma\left(h_{2}\right) \\
& =\beta\left(n_{1}\right) \gamma\left(h_{1}\right) \beta\left(n_{2}\right) \underbrace{\gamma\left(h_{1}^{-1}\right) \gamma\left(h_{1}\right)}_{1_{G}} \gamma\left(h_{2}\right) \\
& =\beta\left(n_{1}\right) \gamma\left(h_{1}\right) \beta\left(n_{2}\right) \gamma\left(h_{2}\right) \\
& =\Omega\left(n_{1}, h_{1}\right) \Omega\left(n_{2}, h_{2}\right) .
\end{aligned}
$$

Vejamos que $\Omega$ é injetivo. De fato, se $\Omega(n, h)=1$, então $\beta(n) \gamma(h)=1$. Aplicando $\alpha$ a ambos lados, desta igualdade obtemos,

$$
\underbrace{\alpha(\beta(n))}_{1_{H}} \underbrace{\alpha(\gamma(h))}_{h}=\alpha(1)=1_{H}
$$

assim, $h=1_{H}$. Então, $\beta(n) \cdot 1=1$, assim $n=1_{N}$, pois $\beta$ é injetor.

Agora, mostremos que $\Omega$ é sobrejetor. Seja $g \in G$ e considere $h:=\alpha(g) \in H$. Note que $g \cdot \gamma\left(\alpha\left(g^{-1}\right)\right) \in \operatorname{Ker}(\alpha)=i m(\beta)$, pois

$$
\alpha\left(g \cdot \gamma\left(\alpha\left(g^{-1}\right)\right)\right)=\alpha(g) \alpha\left(\gamma\left(\alpha\left(g^{-1}\right)\right)\right)=\alpha(g) \alpha\left(g^{-1}\right)=1_{H}
$$


Assim, defina $n:=\beta^{-1}\left(g \cdot \gamma\left(\alpha\left(g^{-1}\right)\right)\right) \in N$. Então,

$$
\begin{aligned}
\Omega(n, h) & =\beta(n) \gamma(h) \\
& =g \cdot \underbrace{\gamma\left(\alpha\left(g^{-1}\right)\right) \gamma(\alpha(g))}_{1} \\
& =g .
\end{aligned}
$$

Portanto $\Omega: N \rtimes_{\varphi} H \longrightarrow G$ é um isomorfismo.

Mostremos agora que o diagrama da Figura 13 é comutativo.

- Comutatividade do quadrado $(I)$. Para qualquer $n \in N$ :

$$
\begin{aligned}
\Omega \circ i_{1}(n) & =\Omega(n, 1) \\
& =\beta(n) \gamma(1) \\
& =\beta(n),
\end{aligned}
$$

$\operatorname{assim} \Omega \circ i_{1}=\beta$.

- Comutatividade do quadrado $(I I)$. Para qualquer $(n, h) \in N \rtimes_{\varphi} H$ :

$$
\begin{aligned}
\alpha \circ \Omega(n, h) & =\alpha(\beta(n) \gamma(h)) \\
& =\underbrace{\alpha(\beta(n))}_{1} \alpha(\gamma(h)) \\
& =\alpha(\gamma(h)) \\
& =h \\
& =\pi(n, h),
\end{aligned}
$$

assim $\alpha \circ \Omega=\pi$. Portanto, segue a comutatividade do Diagrama da Figura 13.

Mostremos que $(i i) \Longrightarrow(i)$. Definimos o homomorfismo $\gamma:=\Omega \circ i_{2}: H \longrightarrow G$ satisfazendo:

$$
\begin{aligned}
\alpha \circ \gamma & =\underbrace{\alpha \circ \Omega}_{(I I)} \circ i_{2} \\
& =\pi \circ i_{2} \\
& =i d_{H} .
\end{aligned}
$$

Assim, a sequência (4.1.2) cinde.

Observação 4.1.8. Note que, se no Lema 4.1.7, consideramos a categoria de grupos abelianos, então o homomorfismo $\varphi: H \longrightarrow A u t(N)$ é trivial, pois:

$$
\varphi_{h}(n)=\beta^{-1}(\underbrace{\gamma(h) \beta(n) \gamma\left(h^{-1}\right)}_{\text {em } G \text { que é abeliano }})=n, \quad \forall h \in H .
$$


Assim, pela Observação 4.1.3

$$
N \rtimes_{\varphi} H=N \oplus H=N \times H
$$

e obtemos o conhecido "Lema da cisão".

Lema 4.1.9 (Lema da cisão). ((SELICK, 2008), Chapter 4, Section 1, Proposition 4.14, pg. 22) Dada uma sequência exata curta de grupos abelianos e homomorfismos de grupos

$$
0 \longrightarrow N \stackrel{\beta}{\longrightarrow} G \stackrel{\alpha}{\longrightarrow} H \longrightarrow 0
$$

as seguintes condições são equivalentes:

(i) A sequência cinde.

(ii) Existe um isomorfismo $\theta: N \oplus H \longrightarrow G$.

Proposição 4.1.10. Dados grupos $M, N$ e $f: N \longrightarrow M$ um isomorfismo de grupos.

$$
\begin{aligned}
\operatorname{Aut}(f): \operatorname{Aut}(N) & \longrightarrow \operatorname{Aut}(M) \\
\varphi & \mapsto \operatorname{Aut}(f)(\varphi):=f \circ \varphi \circ f^{-1}, \quad \forall \varphi \in \operatorname{Aut}(N)
\end{aligned}
$$

é um isomorfismo de grupos.

Demonstração. Aut $(f)$ é bem definido. Além disso, é um homomorfismo de grupos, pois:

$$
\begin{aligned}
\operatorname{Aut}(f)(\varphi \circ \psi) & =f \circ(\varphi \circ \psi) \circ f^{-1} \\
& =\left(f \circ \varphi \circ f^{-1}\right) \circ\left(f \circ \psi \circ f^{-1}\right) \\
& =\operatorname{Aut}(f)(\varphi) \circ \operatorname{Aut}(f)(\psi) .
\end{aligned}
$$

Mais ainda, $A u t(f)$ possui inversa $A u t\left(f^{-1}\right)$, pois:

$$
\begin{aligned}
\left(\operatorname{Aut}(f) \circ \operatorname{Aut}\left(f^{-1}\right)\right)(\varphi) & =\operatorname{Aut}(f)\left(f^{-1} \circ \varphi \circ f\right) \\
& =f \circ\left(f^{-1} \circ \varphi \circ f\right) \circ f^{-1} \\
& =\varphi . \\
\left(\text { Aut }\left(f^{-1}\right) \circ \operatorname{Aut}(f)\right)(\varphi) & =\operatorname{Aut}\left(f^{-1}\right)\left(f \circ \varphi \circ f^{-1}\right) \\
& =f^{-1} \circ\left(f \circ \varphi \circ f^{-1}\right) \circ f \\
& =\varphi .
\end{aligned}
$$

Portanto, Aut $(f): \operatorname{Aut}(N) \longrightarrow \operatorname{Aut}(M)$ é um isomorfismo de grupos. 
Proposição 4.1.11. Seja $\varphi: H \longrightarrow A u t(N)$ um homomorfismo de grupos. Se $f: N \longrightarrow N^{\prime}$ e $g: H \longrightarrow H^{\prime}$ são isomorfismos de grupos, então:

$$
N \rtimes_{\varphi} H \cong N^{\prime} \rtimes_{\varphi^{\prime}} H^{\prime}
$$

onde $\varphi^{\prime}: H^{\prime} \longrightarrow \operatorname{Aut}\left(N^{\prime}\right)$ é definida pela composição:

$$
H^{\prime} \stackrel{g^{-1}}{\longrightarrow} H \stackrel{\varphi}{\longrightarrow} \operatorname{Aut}(N) \stackrel{\text { Aut }(f)}{\longrightarrow} \operatorname{Aut}\left(N^{\prime}\right), \text { ou seja } \varphi^{\prime}:=\operatorname{Aut}(f) \circ \varphi \circ g^{-1} .
$$

Demonstração. Definimos:

$$
\begin{aligned}
\mu: N \times H & \longrightarrow N^{\prime} \times H^{\prime} \\
(n, h) & \mapsto \mu(n, h):=(f(n), g(h)) .
\end{aligned}
$$

Vejamos que $\mu$ é um homomorfismo de grupos. Para todo $\left(n_{1}, h_{1}\right),\left(n_{2}, h_{2}\right) \in N \times H$ :

$$
\begin{aligned}
\mu\left(\left(n_{1}, h_{1}\right) \cdot\left(n_{2}, h_{2}\right)\right) & =\mu\left(n_{1} \varphi_{h_{1}}\left(n_{2}\right), h_{1} h_{2}\right) \\
& =\left(f\left(n_{1} \varphi_{h_{1}}\left(n_{2}\right)\right), g\left(h_{1} h_{2}\right)\right) \\
& =(f\left(n_{1}\right) \underbrace{f\left(\varphi_{h_{1}}\left(n_{2}\right)\right)}, g\left(h_{1}\right) g\left(h_{2}\right)) \\
& =(f\left(n_{1}\right) \underbrace{f\left(\varphi\left(h_{1}\right)\left(n_{2}\right)\right)}, g\left(h_{1}\right) g\left(h_{2}\right)) \\
& =(f\left(n_{1}\right) \underbrace{\left(f \circ \varphi\left(h_{1}\right) \circ f^{-1}\right)\left(f\left(n_{2}\right)\right)}, g\left(h_{1}\right) g\left(h_{2}\right)) \\
& =(f\left(n_{1}\right) \underbrace{\left.\left(A u t\left(h_{1}\right)\right)\right)\left(f\left(n_{2}\right)\right)}_{\left.\varphi^{\prime}\left(g\left(h_{1}\right)\right)\left(f\left(n_{2}\right)\right), g\left(h_{1}\right) g\left(h_{2}\right)\right)}, g\left(h_{1}\right) g\left(h_{2}\right)) \\
& =\left(f\left(n_{1}\right) \varphi_{g\left(h_{1}\right)}^{\prime}\left(f\left(n_{2}\right)\right), g\left(h_{1}\right) g\left(h_{2}\right)\right) \\
& =\left(f\left(n_{1}\right), g\left(h_{1}\right)\right) \cdot\left(f\left(n_{2}\right), g\left(h_{2}\right)\right) \\
& =\mu\left(n_{1}, h_{1}\right) \cdot \mu\left(n_{2}, h_{2}\right) .
\end{aligned}
$$

Consideremos o seguinte homomorfismo de grupos:

$$
\begin{aligned}
\nu: N^{\prime} \times H^{\prime} & \longrightarrow N \times H \\
\left(n^{\prime}, h^{\prime}\right) & \mapsto \nu\left(n^{\prime}, h^{\prime}\right):=\left(f^{-1}\left(n^{\prime}\right), g^{-1}\left(h^{\prime}\right)\right) .
\end{aligned}
$$

Então, para todo $(n, h),\left(n^{\prime}, h^{\prime}\right)$ :

$$
\begin{aligned}
\mu \circ \nu\left(n^{\prime}, h^{\prime}\right) & =\mu\left(f^{-1}\left(n^{\prime}\right), g^{-1}\left(h^{\prime}\right)\right) \\
& =\left(f \circ f^{-1}\left(n^{\prime}\right), g \circ g^{-1}\left(h^{\prime}\right)\right) \\
& =\left(n^{\prime}, h^{\prime}\right) . \\
\nu \circ \mu(n, h) & =\nu(f(n), g(h)) \\
& =\left(f^{-1} \circ f(n), g^{-1} \circ g(h)\right) \\
& =(n, h) .
\end{aligned}
$$

Portanto, $\mu: N \rtimes_{\varphi} H \longrightarrow N^{\prime} \rtimes_{\varphi^{\prime}} H^{\prime}$ é um isomorfismo de grupos. 
Observação 4.1.12. Quando não for essencial no contexto ressaltar qual é o homomorfismo $\varphi$, o produto semi-direito $N \rtimes_{\varphi} H$ será denotado simplesmente por $N \rtimes H$.

Definição 4.1.13. ((KAWAKUBO, 1991), Section 2.3, pg. 87) Dizemos que uma função contínua $p: E \longrightarrow B$ possui uma seção ${ }^{1}$, se existe uma função contínua $s: B \longrightarrow E$ tal que $p \circ s=i d_{B}$. Dizemos ainda que $p$ possui uma seção local em um ponto $b \in B$, se existem uma vizinhança aberta $U$ de $b$ e uma função contínua $s_{b}: U \longrightarrow E$ tal que $p \circ s_{b}=i d_{U}$.

A seguir, mostraremos propriedades importantes de uma fibração, no caso de existência de uma seção. No resultado a seguir, por simplicidade de notação, não especificaremos os pontos bases.

Proposição 4.1.14. Seja $F \hookrightarrow E \stackrel{p}{\longrightarrow} B$ uma fibração e suponhamos que exista uma seção $s: B \longrightarrow E$. Então:

(a) $\partial: \pi_{i+1}(B) \longrightarrow \pi_{i}(F)$ é trivial, $\forall i \geq 0$.

(b) $\pi_{i}(E) \cong \pi_{i}(F) \oplus \pi_{i}(B), \forall i \geq 2$.

(c) $\pi_{1}(E) \cong \pi_{1}(F) \rtimes \pi_{1}(B)$.

Demonstração. Seja $s: B \longrightarrow E$ uma seção para $F \hookrightarrow E \stackrel{p}{\longrightarrow} B$.

(a) Da sequência exata longa em homotopia da fibração (vide Teorema 3.1.22)

$$
\cdots \longrightarrow \pi_{i+1}(E) \underset{s_{\star}}{\stackrel{p_{\star}}{\rightleftarrows}} \pi_{i+1}(B) \stackrel{\partial}{\longrightarrow} \pi_{i}(F) \longrightarrow \cdots
$$

segue que $p_{*} \circ s_{\star}=I d_{B}$, o que implica que $p_{*}$ é sobrejetor e $s_{*}$ é injetor. Assim, pela exatidão da sequência (4.1.5) temos:

$$
\operatorname{Ker}(\partial)=\operatorname{Im}\left(p_{*}\right)=\pi_{i+1}(B)
$$

e, portanto, $\partial$ é o homomorfismo trivial, $\forall i \geq 0$.

(b) Pelo item (a), na sequência (4.1.5), para todo $i \geq 2$ :

$$
\cdots \longrightarrow \pi_{i+1}(B) \stackrel{\partial \equiv 0}{\longrightarrow} \pi_{i}(F) \longrightarrow \pi_{i}(E) \longrightarrow \pi_{i}(B) \stackrel{\partial \equiv 0}{\longrightarrow} \pi_{i-1}(F) \longrightarrow \cdots
$$

obtemos a sequência exata curta de grupos abelianos

$$
0 \longrightarrow \pi_{i}(F) \longrightarrow \pi_{i}(E) \underset{p_{\star}}{\stackrel{p_{\star}}{\rightleftarrows}} \pi_{i}(B) \longrightarrow 0
$$

a qual cinde. Portanto, pelo Lema 4.1.9, $\pi_{i}(E) \cong \pi_{i}(F) \oplus \pi_{i}(B), \forall i \geq 2$. 
(c) Pelo item (a), na sequência (4.1.5)

$$
\cdots \longrightarrow \pi_{2}(B) \stackrel{\partial \equiv 0}{\longrightarrow} \pi_{1}(F) \longrightarrow \pi_{1}(E) \longrightarrow \pi_{1}(B) \stackrel{\partial \equiv 0}{\longrightarrow} \pi_{0}(F) \longrightarrow \cdots
$$

obtemos a sequência exata curta de grupos

$$
1 \longrightarrow \pi_{1}(F) \longrightarrow \pi_{1}(E) \underset{p_{\star}}{\stackrel{s_{\star}}{\rightleftarrows}} \pi_{1}(B) \longrightarrow 1
$$

a qual cinde. Portanto, pelo Lema 4.1.7, $\pi_{1}(E) \cong \pi_{1}(F) \rtimes \pi_{1}(B)$.

Observação 4.1.15. Se $A u t\left(\pi_{1}(F)\right)=\left\{I d_{\pi_{1}(F)}\right\}$, segue da Observação 4.1.4 que:

$$
\pi_{1}(E) \cong \pi_{1}(F) \oplus \pi_{1}(B)
$$

Exemplo 4.1.16. ((COHEN, 2010), Example 4.1, pg. 192) Sejam $M$ um espaço topológico arbitrário e $n \geq 1$ um natural. A aplicação

$$
\pi_{k, k-1}: F\left(M \times \mathbb{R}^{n}, k\right) \longrightarrow F\left(M \times \mathbb{R}^{n}, k-1\right)
$$

possui uma seção

$$
s: F\left(M \times \mathbb{R}^{n}, k-1\right) \longrightarrow F\left(M \times \mathbb{R}^{n}, k\right),
$$

definida por

$$
\begin{gathered}
s\left(\left(m_{1}, x_{1}\right), \ldots,\left(m_{k-1}, x_{k-1}\right)\right):=\left(\left(m_{1}, x_{1}\right), \ldots,\left(m_{k-1}, x_{k-1}\right),\left(m_{1}, L \vec{e}_{1}\right)\right), \text { onde } \\
L=1+\max _{1 \leq i \leq k-1}\left\|x_{i}\right\| \text { e } \vec{e}_{1}=(1,0, \ldots, 0) \in \mathbb{R}^{n} .
\end{gathered}
$$

Observemos que $x_{j} \neq L \vec{e}_{1}, \forall j=1, \ldots, k-1$. De fato, se $x_{j}=L \vec{e}_{1}$, para algum $j=1, \ldots, k-1$, então:

$$
\left\|x_{j}\right\|=L=1+\max _{1 \leq i \leq k-1}\left\|x_{i}\right\| \geq 1+\left\|x_{j}\right\|>\left\|x_{j}\right\|, \text { o que é uma contradição. }
$$

Portanto, $s$ é bem definida. Além disso, $s$ é contínua e $\pi_{k, k-1} \circ s=I d_{F\left(M \times \mathbb{R}^{n}, k-1\right)}$.

Observação 4.1.17. Segue do Exemplo 4.1.16, no caso particular em que $M=\{p t o\}$, que a aplicação

$$
\pi_{k, k-1}: F\left(\mathbb{R}^{n}, k\right) \longrightarrow F\left(\mathbb{R}^{n}, k-1\right)
$$

possui uma seção

$$
s: F\left(\mathbb{R}^{n}, k-1\right) \longrightarrow F\left(\mathbb{R}^{n}, k\right) .
$$

Proposição 4.1.18. Para $M$ uma variedade topológica conexa arbitrária e $n \geq 2, k \geq 1$ :

$$
\pi_{i}\left(F\left(M \times \mathbb{R}^{n}, k+1\right)\right) \cong \bigoplus_{r=0}^{k} \pi_{i}\left(\left(M \times \mathbb{R}^{n}\right)-Q_{r}\right), \forall i \geq 2
$$


Demonstração. Pelo Exemplo 4.1.16, sabemos que a fibração

$$
\left(M \times \mathbb{R}^{n}\right)-Q_{k} \hookrightarrow F\left(M \times \mathbb{R}^{n}, k+1\right) \stackrel{\pi_{k+1} k}{\longrightarrow} F\left(M \times \mathbb{R}^{n}, k\right)
$$

possui uma seção. Logo, pela Proposição 4.1.14-(b)

$$
\pi_{i}\left(F\left(M \times \mathbb{R}^{n}, k+1\right)\right) \cong \pi_{i}\left(\left(M \times \mathbb{R}^{n}\right)-Q_{k}\right) \oplus \pi_{i}\left(F\left(M \times \mathbb{R}^{n}, k\right)\right), \forall i \geq 2 .
$$

Usando este fato, o resultado seguirá por indução sobre $k \geq 1$. Para $k=1$ segue de (4.1.6). Suponhamos que o resultado seja válido para qualquer $1 \leq l \leq k(\star)$.

Novamente, de (4.1.6) e usando a hipótese de indução, segue que:

$$
\begin{gathered}
\pi_{i}\left(F\left(M \times \mathbb{R}^{n}, k+1\right)\right) \cong \pi_{i}\left(\left(M \times \mathbb{R}^{n}\right)-Q_{k}\right) \oplus \underbrace{\pi_{i}\left(F\left(M \times \mathbb{R}^{n}, k\right)\right)}_{(\star)} \\
\cong \pi_{i}\left(\left(M \times \mathbb{R}^{n}\right)-Q_{k}\right) \oplus\left(\bigoplus_{r=0}^{k-1} \pi_{i}\left(\left(M \times \mathbb{R}^{n}\right)-Q_{r}\right)\right) \cong \bigoplus_{r=0}^{k} \pi_{i}\left(\left(M \times \mathbb{R}^{n}\right)-Q_{r}\right), \quad \forall i \geq 2 .
\end{gathered}
$$

Portanto,

$$
\pi_{i}\left(F\left(M \times \mathbb{R}^{n}, k+1\right)\right) \cong \bigoplus_{r=0}^{k} \pi_{i}\left(\left(M \times \mathbb{R}^{n}\right)-Q_{r}\right), \forall i \geq 2 .
$$

Proposição 4.1.19. Para $M$ uma variedade topológica conexa com $\operatorname{dim}(M) \geq 1$ e para naturais $n \geq 2$ e $k \geq 1$ :

$$
\pi_{1}\left(F\left(M \times \mathbb{R}^{n}, k+1\right)\right) \cong \underbrace{\pi_{1}(M) \rtimes\left(\pi_{1}(M) \rtimes\left(\cdots \rtimes\left(\pi_{1}(M) \rtimes \pi_{1}(M)\right) \cdots\right)\right)}_{k+1} .
$$

Demonstração. Pelo Exemplo 4.1.16, sabemos que a fibração

$$
\left(M \times \mathbb{R}^{n}\right)-Q_{k} \hookrightarrow F\left(M \times \mathbb{R}^{n}, k+1\right) \stackrel{\pi_{k+1} k}{\longrightarrow} F\left(M \times \mathbb{R}^{n}, k\right)
$$

possui uma seção. Logo, pela Proposição 4.1.14-(c)

$$
\pi_{1}\left(F\left(M \times \mathbb{R}^{n}, k+1\right)\right) \cong \pi_{1}\left(\left(M \times \mathbb{R}^{n}\right)-Q_{k}\right) \rtimes \pi_{1}\left(F\left(M \times \mathbb{R}^{n}, k\right)\right) .
$$

Aplicando este mesmo argumento de forma iterada, obtemos:

$$
\begin{gathered}
\pi_{1}\left(F\left(M \times \mathbb{R}^{n}, k+1\right)\right) \cong \pi_{1}\left(\left(M \times \mathbb{R}^{n}\right)-Q_{k}\right) \rtimes\left(\pi_{1}\left(\left(M \times \mathbb{R}^{n}\right)-Q_{k-1}\right) \rtimes\right. \\
\left.\left(\cdots \rtimes\left(\pi_{1}\left(\left(M \times \mathbb{R}^{n}\right)-Q_{1}\right) \rtimes \pi_{1}\left(M \times \mathbb{R}^{n}\right)\right) \cdots\right)\right) .
\end{gathered}
$$

Agora, como $\operatorname{dim}\left(M \times \mathbb{R}^{n}\right) \geq 3$, segue do Exemplo B.1.5 que

$$
\pi_{1}\left(\left(M \times \mathbb{R}^{n}\right)-Q_{i}\right) \cong \pi_{1}\left(M \times \mathbb{R}^{n}\right), \quad \forall i .
$$

Além disso, $M \times \mathbb{R}^{n}$ tem o mesmo tipo de homotopia de $M$, logo:

$$
\pi_{1}\left(F\left(M \times \mathbb{R}^{n}, k+1\right)\right) \cong \underbrace{\pi_{1}(M) \rtimes\left(\pi_{1}(M) \rtimes\left(\cdots \rtimes\left(\pi_{1}(M) \rtimes \pi_{1}(M)\right) \cdots\right)\right)}_{k+1} .
$$


Observação 4.1.20. Consideremos o produto semi-direto $G:=N \rtimes_{\varphi} H$ e sejam

$$
g_{1}=\left(n_{1}, h_{1}\right), \quad g_{2}=\left(n_{2}, h_{2}\right) \in G \text {. }
$$

Então, o comutador de $g_{1}, g_{2}$ é dado por:

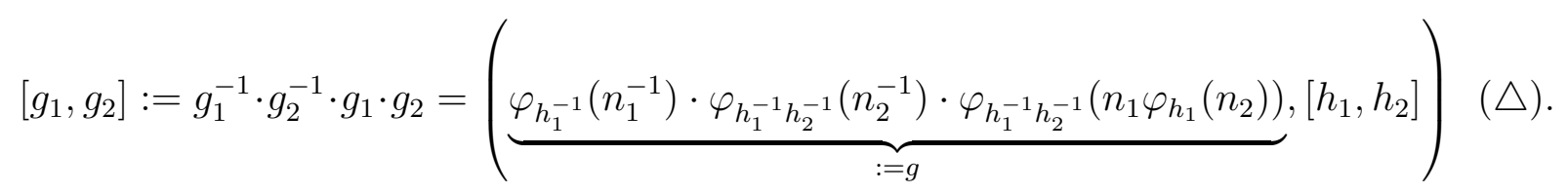

Assim, se $H$ for um grupo abeliano não trivial, então $\left[g_{1}, g_{2}\right]=\left(g, 1_{H}\right)$, onde $g$ é como em $(\triangle)$. Portanto, o subgrupo comutador

$$
[G, G]:=\langle S\rangle, \text { com } S:=\{[g, h]: g, h \in G\}
$$

é tal que $[G, G] \neq G$, ou seja, o grupo quociente $G /[G, G]$ é não trivial. Dessa forma, a abelianização $G /[G, G]$ do grupo $G$ é não trivial.

Observação 4.1.21. Consideremos o produto semi-direto iterado:

$$
G:=N_{1} \rtimes\left(N_{2} \rtimes\left(N_{3} \rtimes \cdots\left(N_{k} \rtimes N_{k+1}\right) \cdots\right)\right)=\left(N_{1} \times \cdots \times N_{k+1}, \cdot\right)
$$

e sejam $g=\left(g_{1}, \ldots, g_{k}, g_{k+1}\right), \quad h=\left(h_{1}, \ldots, h_{k}, h_{k+1}\right) \in G$. Então, aplicando os mesmos cálculos apresentados na Observação 4.1.20, o comutador de $g$ e $h$ tem a forma:

$$
[g, h]=\left(m_{1}, \ldots, m_{k},\left[g_{k+1}, h_{k+1}\right]\right) \text {. }
$$

Assim, se $N_{k+1}$ for um grupo abeliano não trivial, então $[g, h]=\left(m_{1}, \ldots, m_{k}, 1_{k+1}\right)$, onde $m_{i} \in N_{i}$. Portanto, o subgrupo comutador

$$
[G, G]:=\langle S\rangle, \text { com } S:=\{[g, h]: g, h \in G\}
$$

é tal que $[G, G] \neq G$, ou seja, o grupo quociente $G /[G, G]$ é não trivial. Dessa forma, a abelianização $G /[G, G]$ do grupo $G$ é não trivial.

Neste sentido, obtemos o seguinte resultado.

Corolário 4.1.22. Sejam $M$ uma variedade topológica conexa, $\operatorname{com} \operatorname{dim}(M) \geq 1$ e naturais $n \geq 2$ e $k \geq 1$. Se $\pi_{1}(M)$ for um grupo abeliano não trivial, então:

(i) $H_{1}\left(F\left(M \times \mathbb{R}^{n}, k+1\right)\right)$ é não trivial.

(ii) $\pi_{1}\left(F\left(M \times \mathbb{R}^{n}, k+1\right)\right)$ é não trivial.

Demonstração. Para a variedade conexa $M$ e para quaisquer $n \geq 2$ e $k \geq 1$, temos pela Proposição 4.1.19 o seguinte isomorfismo:

$$
\pi_{1}\left(F\left(M \times \mathbb{R}^{n}, k+1\right)\right) \cong \underbrace{\pi_{1}(M) \rtimes\left(\pi_{1}(M) \rtimes\left(\cdots \rtimes\left(\pi_{1}(M) \rtimes \pi_{1}(M)\right) \cdots\right)\right)}_{k+1} .
$$


(i) Sabemos da Observação 3.1.28 que $F\left(M \times \mathbb{R}^{n}, k+1\right)$ é 0-conexo. Como $\pi_{1}(M)$ é um grupo abeliano não trivial, segue da Observação 4.1.20 e do Teorema de Hurewicz (vide Teorema B.1.9-(b)) que:

$$
H_{1}\left(F\left(M \times \mathbb{R}^{n}, k+1\right)\right) \cong G /[G, G] \neq 0, \quad \operatorname{com} G=\pi_{1}\left(F\left(M \times \mathbb{R}^{n}, k+1\right)\right) .
$$

(ii) Como $\pi_{1}(M)$ é não trivial, existe $g \in \pi_{1}(M)$ tal que $g \neq 1$. Então,

$$
(g, 1, \cdots, 1) \neq 1 \in \underbrace{\pi_{1}(M) \rtimes\left(\pi_{1}(M) \rtimes\left(\cdots \rtimes\left(\pi_{1}(M) \rtimes \pi_{1}(M)\right) \cdots\right)\right)}_{k+1} \cong G,
$$

$\operatorname{com} G=\pi_{1}\left(F\left(M \times \mathbb{R}^{n}, k+1\right)\right)$. Portanto, $\pi_{1}\left(F\left(M \times \mathbb{R}^{n}, k+1\right)\right)$ é não trivial.

Exemplo 4.1.23. Se $M$ é a 1-esfera $\mathbb{S}^{1}$, pela Proposição 4.1.19 temos que:

$$
\begin{aligned}
\pi_{1}\left(F\left(\mathbb{S}^{1} \times \mathbb{R}^{2}, k+1\right)\right) & \cong \underbrace{\pi_{1}\left(\mathbb{S}^{1}\right) \rtimes\left(\pi_{1}\left(\mathbb{S}^{1}\right) \rtimes\left(\cdots \rtimes\left(\pi_{1}\left(\mathbb{S}^{1}\right) \rtimes \pi_{1}\left(\mathbb{S}^{1}\right)\right) \cdots\right)\right)}_{k+1} \\
& \cong \underbrace{\mathbb{Z} \rtimes(\mathbb{Z} \rtimes(\cdots \rtimes(\mathbb{Z} \rtimes \mathbb{Z}) \cdots))}_{k+1}, \text { pois } \pi_{1}\left(\mathbb{S}^{1}\right) \cong \mathbb{Z} .
\end{aligned}
$$

Definição 4.1.24. O grupo ortogonal $O(n)$ é o grupo das matrizes ortogonais com entradas reais. O grupo ortogonal especial $S O(n)=O(n) \cap S L(n, \mathbb{R})$, onde $S L(n, \mathbb{R})$ denota o grupo linear especial das matrizes com entradas reais e com determinante igual a 1, ou seja, $S O(n)$ é o subgrupo de $O(n)$ das matrizes ortogonais com entradas reais e determinante igual a 1. (vide (DIECK, 1987), Chapter 1, Section 2, pg. 11).

Exemplo 4.1.25. Mostremos que se $M$ é o grupo ortogonal especial $S O(n)$, então:

$$
H_{1}\left(F\left(S O(n) \times \mathbb{R}^{n}, k+1\right), \mathbb{Z}\right) \cong \pi_{1}\left(F\left(S O(n) \times \mathbb{R}^{n}, k+1\right)\right) \cong \bigoplus_{1}^{k+1} \mathbb{Z}_{2}, \quad \forall n \geq 3,
$$

De fato, pela Proposição 4.1.19:

$\pi_{1}\left(F\left(S O(n) \times \mathbb{R}^{n}, k+1\right)\right) \cong \underbrace{\pi_{1}(S O(n)) \rtimes\left(\pi_{1}(S O(n)) \rtimes\left(\cdots \rtimes\left(\pi_{1}(S O(n)) \rtimes \pi_{1}(S O(n))\right) \cdots\right)\right)}_{k+1}$.

Como $\pi_{1}(S O(n)) \cong \mathbb{Z}_{2}, \forall n \geq 3$, (vide (HATCHER, 2002), Chapter 3, Section 3.D, Exercise 2, pg. 302) isso implica que $\operatorname{Aut}\left(\pi_{1}(S O(n))\right)=\{I d\}$. Assim, da Observação 4.1.4:

$$
\left.\pi_{1}\left(F\left(S O(n) \times \mathbb{R}^{n}, k+1\right)\right) \cong \bigoplus_{1}^{k+1} \mathbb{Z}_{2} \quad \text { (grupo abeliano }\right)
$$

Portanto, pelo Teorema de Hurewicz (vide Teorema B.1.9):

$$
H_{1}\left(F\left(S O(n) \times \mathbb{R}^{n}, k+1\right), \mathbb{Z}\right) \cong \bigoplus_{1}^{k+1} \mathbb{Z}_{2}
$$


No resultado a seguir, mostraremos que o grupo de tranças puras de Artin $P B_{k+1}$ é isomorfo a um produto semidireto iterado de grupos livres. Este fato é muito interessante para se obter uma ordenação sobre $P B_{k+1}$.

Proposição 4.1.26. ((DEHORNOY et al., 2008), Chapter $X V$, Section2, Proposition 2.4, pg. 264) Para cada $k \geq 1$, o grupo de tranças puras de Artin é isomorfo um produto semi-direto iterado de grupos livres, ou seja:

$$
P B_{k+1} \cong F_{k} \rtimes\left(F_{k-1} \rtimes\left(\cdots \rtimes F_{1} \cdots\right)\right),
$$

onde $F_{k}$ é um grupo livre de posto $k$.

Demonstração. Pelo Teorema 3.1.38, $F\left(\mathbb{R}^{2}, k+1\right)$ é um $K(\pi, 1)$ - espaço. Seja

$$
\mathbb{R}^{2}-\{k \text { pontos }\} \longrightarrow F\left(\mathbb{R}^{2}, k+1\right) \stackrel{\pi_{k+1} k}{\longrightarrow} F\left(\mathbb{R}^{2}, k\right) .
$$

a fibração de Fadell e Neuwirth, na qual a fibra $\mathbb{R}^{2}-\{k$ pontos $\}$ tem o mesmo tipo de homotopia de um buquê de esferas $\bigvee^{k} \mathbb{S}^{1}$ (vide Corolário B.4.2). Consideremos a sequência exata longa em homotopia associada a esta fibração:

$\cdots \longrightarrow \underbrace{\pi_{2}\left(F\left(\mathbb{R}^{2}, k\right)\right)}_{1} \longrightarrow \pi_{1}\left(\bigvee^{k} \mathbb{S}^{1}\right) \longrightarrow \pi_{1}\left(F\left(\mathbb{R}^{2}, k+1\right)\right) \longrightarrow \pi_{1}\left(F\left(\mathbb{R}^{2}, k\right)\right) \longrightarrow \underbrace{\pi_{0}\left(\bigvee^{k} \mathbb{S}^{1}\right)}_{1} \longrightarrow \cdots$

Lembrando que $\pi_{1}\left(\bigvee^{k} \mathbb{S}^{1}\right) \cong F_{k}$ é um grupo livre de posto $k$ e que, pela Definição 3.2.1,

$$
\pi_{1}\left(F\left(\mathbb{R}^{2}, k+1\right)\right)=P B_{k+1} \text { e } \pi_{1}\left(F\left(\mathbb{R}^{2}, k\right)\right)=P B_{k},
$$

obtemos uma sequência exata curta de grupos fundamentais:

$$
1 \longrightarrow F_{k} \longrightarrow P B_{k+1} \longrightarrow P B_{k} \longrightarrow 1
$$

Pelo Exemplo 4.1.16, temos que a projeção $\pi_{k+1, k}: F\left(\mathbb{R}^{2}, k+1\right) \longrightarrow F\left(\mathbb{R}^{2}, k\right)$ possui uma seção $s: F\left(\mathbb{R}^{2}, k\right) \longrightarrow F\left(\mathbb{R}^{2}, k+1\right)$, ou seja, $\pi_{k+1, k} \circ s=I d$. Logo, da Definição 4.1.5, a sequência (4.1.9) cinde. Assim, pelo Lema 4.1.7,

$$
P B_{k+1} \cong F_{k} \rtimes P B_{k} .
$$

Aplicando este mesmo argumento de forma iterada, concluímos que

$$
P B_{k+1} \cong F_{k} \rtimes\left(F_{k-1} \rtimes\left(\cdots \rtimes F_{1} \cdots\right)\right) .
$$

Os resultados obtidos anteriormente, revelam a importância em se conhecer se uma fibração admite seções. Por essa razão, estudaremos a seguir condições que garantem quando a fibração de Fadell e Neuwirth admite seções. Seguindo a mesma notação de (FADELL; NEUWIRTH, 1962), denotaremos a projeção $\pi_{n, 1}$ simplesmente por $\pi$. 
Teorema 4.1.27. ((FADELL; NEUWIRTH, 1962), Theorem 1, pg. 112) Seja $M$ uma variedade topológica conexa, com $\operatorname{dim} M \geq 2$. Se $m \geq 1$, então a projeção:

$$
\pi: F\left(M-Q_{m}, n\right) \longrightarrow M-Q_{m}, \text { onde } Q_{m}:=\left\{q_{1}, \ldots, q_{m}\right\}
$$

admite uma seção.

Demonstração. Consideremos $(\bar{V}, \varphi)$ uma carta para $M$ tal que $\bar{V}$ é vizinhança de disco ${ }^{2}$ em $M$ para $q_{1} \in V$, de tal forma que $q_{j} \notin \bar{V}, \forall 2 \leq j \leq m$. Denotemos por $W=B[0,1 / 2]$ o disco fechado de centro 0 e raio $1 / 2$, e consideremos $y_{2}, \ldots, y_{n} \in \partial W, n-1$ pontos distintos no bordo de $W$.

Para cada $i=2, \ldots, n$ definimos as aplicações $f_{i}: M-Q_{m} \longrightarrow M-Q_{m}$, pela expressão:

$$
x \mapsto f_{i}(x)= \begin{cases}\varphi^{-1}\left(\|\varphi(x)\| y_{i}\right), & \text { se } x \in \bar{V}-\left\{q_{1}\right\} . \\ \varphi^{-1}\left(y_{i}\right), & \text { se } x \notin V .\end{cases}
$$

Note que $\bar{V}-\left\{q_{1}\right\}$ e $\left(M-Q_{m}\right)-V$ são ambos fechados em $M-Q_{m}$, pois:

$$
\begin{aligned}
\bar{V}-\left\{q_{1}\right\} & =\bar{V} \cap\left(M-Q_{m}\right) \\
\left(M-Q_{m}\right)-\left[\left(M-Q_{m}\right)-V\right] & =V \cap\left(M-Q_{m}\right), \text { é aberto em } M-Q_{m} .
\end{aligned}
$$

Cada $f_{i}$ é bem definida sobre o fechado $\bar{V}-\left\{q_{1}\right\}$ de $M-Q_{m}$. De fato, para cada $x \in \bar{V}-\left\{q_{1}\right\}$ :

$$
\|\| \varphi(x)\left\|y_{i}\right\|=\underbrace{\|\varphi(x)\|}_{\leq 1} \underbrace{\left\|y_{i}\right\|}_{=1 / 2} \leq 1 / 2 \leq 1 .
$$

Note também que cada $f_{i}$ sobre $\left(M-Q_{m}\right)-V$ é a constante $\varphi^{-1}\left(y_{i}\right)$, ou seja,

$$
f_{i}(x)=\varphi^{-1}\left(y_{i}\right), \forall x \in\left(M-Q_{m}\right)-V .
$$

Agora, como $y_{i} \in \partial W$, então $\varphi^{-1}\left(y_{i}\right) \in M-Q_{m}$. Assim, $f_{i}$ é bem definida sobre o fechado $\left(M-Q_{m}\right)-V$ de $M-Q_{m}$.

Além disso, na intersecção

$$
\left(\bar{V}-\left\{q_{1}\right\}\right) \cap\left(\left(M-Q_{m}\right)-V\right)=\partial V, \text { onde } \partial V \text { denota o bordo de } V \text {, }
$$

as duas funções que definem $f_{i}$ são iguais, pois

$$
\|\varphi(x)\|=1, \forall x \in \partial V
$$

Portanto, $f_{i}$ é uma aplicação contínua.

2 Vide Observação 3.1.10. 
Seja $f_{1}=I d$ a aplicação identidade em $M-Q_{m}$ e note que para cada $x \in M-Q_{m}$,

$$
\left(f_{1}(x), f_{2}(x), \ldots, f_{n}(x)\right) \in F\left(M-Q_{m}, n\right)
$$

pois as aplicações $f_{2}, \ldots, f_{n}$ são livres de pontos fixos e não são coincidentes. Portanto,

$$
f: M-Q_{m} \longrightarrow F\left(M-Q_{m}, n\right), \quad x \mapsto f(x)=\left(f_{1}(x), f_{2}(x), \ldots, f_{n}(x)\right)
$$

é uma seção para $\pi$.

Da prova do Teorema 4.1.27 podemos obter o seguinte resultado.

Proposição 4.1.28. ((FADELL; NEUWIRTH, 1962), Observation, pg. 113) A projeção $\pi: F\left(M-Q_{m}, n\right) \longrightarrow M-Q_{m}$ admite uma seção se, e somente se, existem $(n-1)$ aplicações livres de pontos fixos:

$$
f_{2}, \ldots, f_{n}: M-Q_{m} \longrightarrow M-Q_{m}
$$

as quais não são coincidentes, ou seja, $f_{i}(x) \neq f_{j}(x), \quad \forall i \neq j$ e $\forall x \in M-Q_{m}$.

Demonstração. A condição suficiente segue da prova do Teorema 4.1.27 e a condição necessária segue da definição de seção (vide Definição 4.1.13).

Corolário 4.1.29. ((FADELL; NEUWIRTH, 1962), Section II, pg. 113) Se uma variedade topológica $M$ possui a propriedade do ponto fixo, ou seja, para qualquer aplicação contínua $f: M \longrightarrow M$, existe $x \in M$ tal que $f(x)=x$. Então, a projeção $\pi: F(M, n) \longrightarrow M$ não admite uma seção.

Exemplo 4.1.30. ((FADELL; NEUWIRTH, 1962), Section II, pg. 113) O plano real projetivo $\mathbb{R P}^{2}$ possui a propriedade do ponto fixo.

Corolário 4.1.31. ((FADELL; NEUWIRTH, 1962), Section II, pg. 113) Seja $M$ uma variedade topológica conexa com $\operatorname{dim} M \geq 2$. Se $m \geq 1$, então a variedade $M-Q_{m}$ não tem a propriedade do ponto fixo.

Considerando a sequência fundamental de fibrações representada no diagrama da Figura 14, obtemos o Corolário 4.1.32. 
Figura 14 - Sequência fundamental de fibrações.
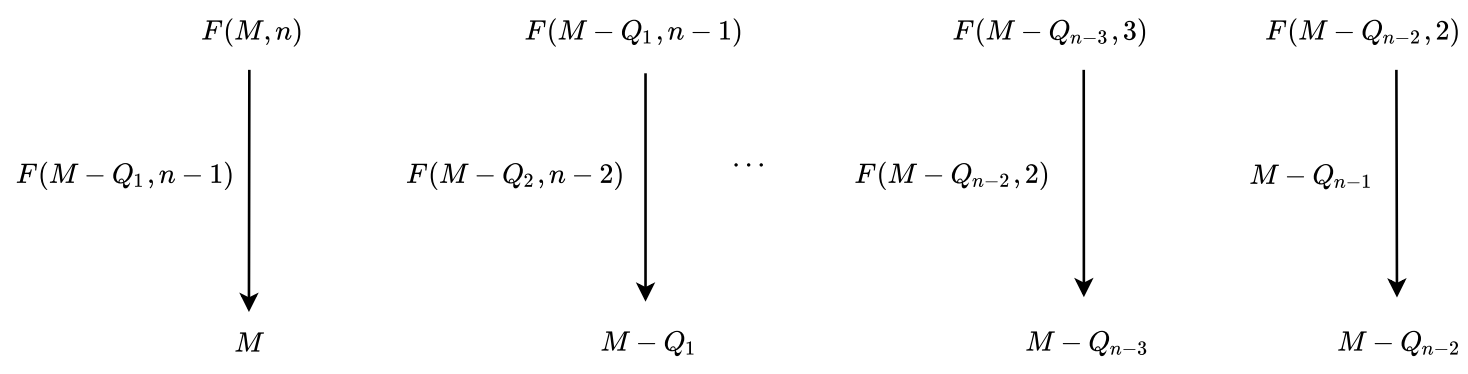

Fonte: Elaborada pelo autor.

Corolário 4.1.32. ((FADELL; NEUWIRTH, 1962), Theorem 2, pg. 113) Seja $M$ uma variedade topológica conexa $M$ arbitrária, com $\operatorname{dim} M \geq 2$. Então, para todo $i \geq 2$ :

$$
\left.\pi_{i}\left(F\left(M-Q_{1}, n-1\right)\right) \cong \bigoplus_{k=1}^{n-1} \pi_{i}\left(M-Q_{k}\right) \quad \text { (soma direta }\right)
$$

Se $\pi: F(M, n) \longrightarrow M$, com fibra $F\left(M-Q_{1}, n-1\right)$, admite uma seção, então

$$
\pi_{i}(F(M, n)) \cong \bigoplus_{k=0}^{n-1} \pi_{i}\left(M-Q_{k}\right), \quad \forall i \geq 2
$$

Demonstração. O resultado segue do Teorema 4.1.27 e da hipótese que $\pi: F(M, n) \longrightarrow M$ admite seção, aplicando sucessivamente a Proposição 4.1.14-(b) à sequência fundamental de fibrações da Figura 14.

A seguir, vamos relacionar as seções das aplicações $\pi_{n, 1}: F(M, n) \longrightarrow M \mathrm{e}$ $\pi_{n, n-1}: F(M, n) \longrightarrow F(M, n-1)$. Para isso, consideremos o diagrama comutativo dado na Figura 15.

Figura 15 - Relação de seções

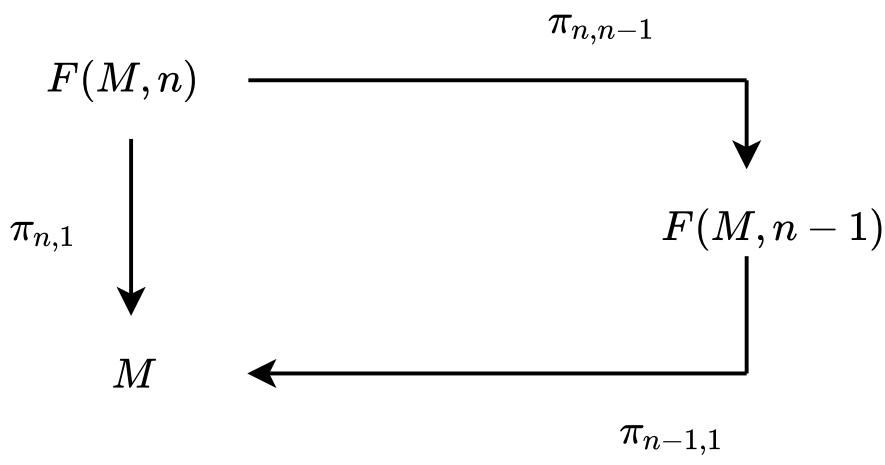

Fonte: Elaborada pelo autor.

A idéia do resultado a seguir foi obtida em (FADELL; NEUWIRTH, 1962), pg. 116. 
Proposição 4.1.33. Consideremos as aplicações:

$$
\pi_{n, 1}: F(M, n) \longrightarrow M \text { e } \pi_{n, n-1}: F(M, n) \longrightarrow F(M, n-1)
$$

1. Se $\pi_{n, 1}$ admite uma seção, então $\pi_{n-1,1}$ admite uma seção.

2. Se $\pi_{n, n-1}$ admite uma seção, para cada $n \geq 2$, então $\pi_{n, 1}$ admite uma seção, $\forall n \geq 2$.

Demonstração. Para o item (1), suponhamos que $\pi_{n, 1}: F(M, n) \longrightarrow M$ admite uma seção $\sigma: M \longrightarrow F(M, n)$. Então,

$$
\pi_{n-1,1} \circ\left(\pi_{n, n-1} \circ \sigma\right)=\left(\pi_{n-1,1} \circ \pi_{n, n-1}\right) \circ \sigma=\pi_{n, 1} \circ \sigma=I d .
$$

O item (2) segue por indução sobre $n$. 
CAPÍTULO

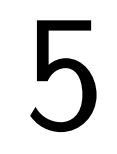

\section{VARIEDADES DISTINTAS COM OS} MESMOS ESPAÇOS DE CONFIGURAÇÕES

Uma questão natural que surge sobre os espaços de configurações é formulada como segue.

"Sejam $M$ e $N$ espaços topológicos arbitrários não vazios. Se $F(M, k)$ e $F(N, k)$ são do mesmo tipo, $\forall k \geq 2$ (ou seja, são homeomorfos, ou possuem o mesmo tipo de homotopia, ou possuem o mesmo tipo de homotopia fraca), então $M$ e $N$ são do mesmo tipo?".

Uma resposta trivial a essa questão, é obtida quando consideramos os espaços $\{p t o\}$ e $\{p t o, p t o\}$. Para tais espaços, claramente não homeomorfos, tem-se:

$$
F(\{p t o\}, k)=\emptyset=F(\{p t o, p t o\}, k), \quad \forall k \geq 3
$$

Observemos ainda que: $F(X, 2)=\emptyset$, implica que $X=\emptyset$ ou $X=\{p t o\}$.

\section{1 $F(M, 2) \simeq F(N, 2)$ não implica $M \simeq N$}

Vamos analisar o caso em que $F(M, 2)$ e $F(N, 2)$ são do mesmo tipo, para espaços topológicos não vazios $M$ e $N$. Uma resposta não trivial neste caso será dada pelos exemplos que apresentaremos na sequência. A seguir, definimos o conceito de equivalência de homotopia fraca.

Definição 5.1.1. ((HATCHER, 2002), Chapter 4, Section 4.1, pg. 352) Uma função contínua $f: X \longrightarrow Y$ é chamada uma equivalência de homotopia fraca se $f$ induz isomorfismos entre os grupos de homotopia:

$$
f_{\star}: \pi_{n}\left(X, x_{0}\right) \longrightarrow \pi_{n}\left(Y, f\left(x_{0}\right)\right)
$$

para $\forall n \geq 0$ e para todas as escolhas de pontos base $x_{0} \in X$. 
Exemplo 5.1.2. Mostremos que $S F\left(\mathbb{S}^{n}, 2\right)$ tem o mesmo tipo de homotopia que o espaço projetivo real $\mathbb{R P}^{n}$, para todo $n \geq 1$.

Demonstração. No Exemplo 2.1.10-(6), mostramos que a aplicação

$$
f: \mathbb{S}^{n} \longrightarrow F\left(\mathbb{S}^{n}, 2\right)
$$

definida por $f(x)=(x,-x)$ é uma equivalência de homotopia. Sabemos também que $\mathbb{S}^{n}$ e $F\left(\mathbb{S}^{n}, 2\right)$ são $S_{2}$-espaços livres, onde $S_{2}$ denota o grupo simétrico (vide Proposição 2.1.4). Além disso, $f$ é uma aplicação $S_{2}$-equivariante, pois

$$
((-1) \cdot x)=f(-x)=(-x, x)=(-1) \cdot(x,-x)=(-1) \cdot f(x), \forall x \in \mathbb{S}^{n} .
$$

Assim, $f$ induz uma aplicação entre os espaços quocientes $\bar{f}: \mathbb{R} \mathbb{P}^{n} \longrightarrow S F\left(\mathbb{S}^{n}, 2\right)$ e obtemos o diagrama comutativo dado na Figura 16.

Figura $16-S F\left(\mathbb{S}^{n}, 2\right) \simeq \mathbb{R} \mathbb{P}^{n}$

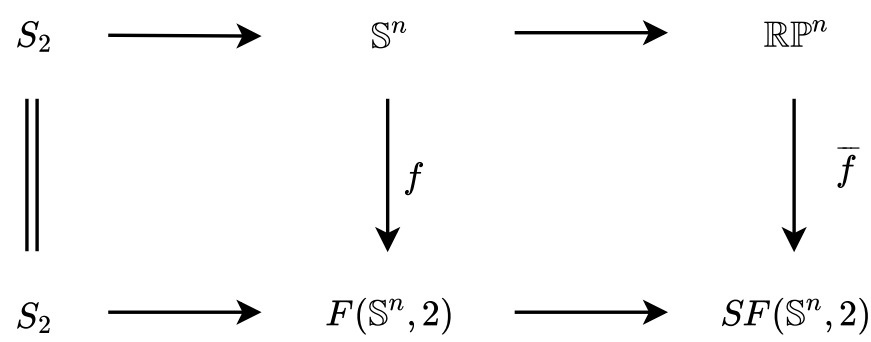

Fonte: Elaborada pelo autor.

Como $f$ é uma equivalência de homotopia, segue que $\bar{f}$ é uma equivalência de homotopia fraca (vide Proposição B.3.4). Além disso, $\mathbb{R P}^{n}=F\left(\mathbb{R P}^{n}, 1\right)$ e $S F\left(\mathbb{S}^{n}, 2\right)$ são complexos CW (vide Corolário 2.1.21), pois $\mathbb{R P}^{n}$ e $\mathbb{S}^{n}$ são variedades suaves. Assim, a aplicação quociente $\bar{f}: \mathbb{R P}^{n} \longrightarrow S F\left(\mathbb{S}^{n}, 2\right)$ é uma equivalência de homotopia fraca entre complexos CW, logo $\bar{f}$ é uma equivalência de homotopia (vide (WHITEHEAD, 1978), Chapter V, Section 3, Theorem 3.5, pag. 220).

Exemplo 5.1.3. Mostremos que $S F\left(\mathbb{R}^{n+1}, 2\right)$ tem o mesmo tipo de homotopia do espaço projetivo real $\mathbb{R P}^{n}$ (vide (COHEN, 1973), Proposition III, pg. 764 e (COHEN, 1978), Proposition 5.5, pg. 248, 256).

Demonstração. Mostramos no Exemplo 2.1.10-(2), que a aplicação

$$
f: \mathbb{S}^{n} \longrightarrow F\left(\mathbb{R}^{n+1}, 2\right), \quad f(x)=(x,-x)
$$

é uma equivalência homotópica. Sabemos também que $\mathbb{S}^{n}$ e $F\left(\mathbb{R}^{n+1}, 2\right)$ são $S_{2}$-espaços livres, onde $S_{2}$ denota o grupo simétrico (vide Proposição 2.1.4). Além disso, $f$ é uma aplicação $S_{2}$-equivariante, pois

$$
f((-1) \cdot x)=f(-x)=(-x, x)=(-1) \cdot(x,-x)=(-1) \cdot f(x), \quad \forall x \in \mathbb{S}^{n} .
$$


Logo, $f$ induz uma aplicação entre os espaços quocientes $\bar{f}: \mathbb{R} \mathbb{P}^{n} \longrightarrow S F\left(\mathbb{R}^{n+1}, 2\right)$. Assim, obtemos o diagrama comutativo dado na Figura 17.

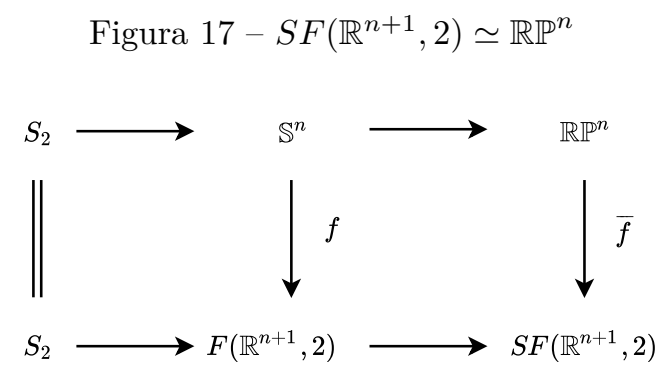

Fonte: Elaborada pelo autor.

Como $f$ é uma equivalência homotópica, segue que $\bar{f}$ é uma equivalência de homotopia fraca (vide Proposição B.3.4). Além disso, $\mathbb{R P}^{n}=F\left(\mathbb{R P}^{n}, 1\right)$ e $S F\left(\mathbb{R}^{n+1}, 2\right)$ são complexos CW (vide Corolário 2.1.21), pois $\mathbb{R P}^{n}$ e $\mathbb{R}^{n+1}$ são variedades suaves. Assim, a aplicação $\bar{f}: \mathbb{R P}^{n} \longrightarrow S F\left(\mathbb{R}^{n+1}, 2\right)$ é uma equivalência de homotopia fraca entre complexos CW, logo $\bar{f}$ é uma equivalência homotópica (vide (WHITEHEAD, 1978), Chapter V, Section 3, Theorem 3.5, pag. 220).

Observação 5.1.4. Portanto,

$$
F\left(\mathbb{S}^{n}, 2\right) \simeq \mathbb{S}^{n} \simeq F\left(\mathbb{R}^{n+1}, 2\right) \text { e } S F\left(\mathbb{S}^{n}, 2\right) \simeq \mathbb{R} P^{n} \simeq S F\left(\mathbb{R}^{n+1}, 2\right)
$$

No entanto, $\mathbb{S}^{n}$ e $\mathbb{R}^{n+1}$ não possuem o mesmo tipo de homotopia.

\subsection{Espaços de configurações para variedades contráteis}

Uma outra resposta parcial para a questão formulada inicialmente pode ser obtida para variedades abertas.

Lema 5.2.1. Sejam $W$ uma $n$-variedade contrátil, sem bordo e $O \subseteq W$ um aberto homeomorfo a $\mathbb{R}^{n}$, com $n \geq 3$. Seja $Q$ qualquer subconjunto finito de $O$. Então, a inclusão

$$
i: O \backslash Q \hookrightarrow W \backslash Q
$$

é uma equivalência de homotopia fraca.

Demonstração. Temos que $O, W \backslash Q$ são conexos por caminhos e $O \cap(W \backslash Q)=O \backslash Q$ é simplesmente conexo, pois tem o mesmo tipo de homotopia de um wedge de $(n-1)$-esferas, com $n-1 \geq 2$. Assim, aplicando o teorema de Seifert-van Kampen (vide Teorema B.1.4) para a cobertura aberta de

$$
W=O \cup(W \backslash Q),
$$


segue que

$$
\{1\} \cong \pi_{1}(W) \cong \pi_{1}(W \backslash Q) * \pi_{1}(O) \cong \pi_{1}(W \backslash Q),
$$

onde $*$ denota o produto livre. Logo, $W \backslash Q$ é simplesmente conexo.

Por outro lado, aplicando a sequência de Mayer-Vietoris (vide Teorema B.1.6), para a mesma cobertura de $W$ pelos abertos $O$ e $W \backslash Q$, a contratibilidade de $W$ e $O$ implica que a inclusão $i_{1}: O \backslash Q \hookrightarrow W \backslash Q$ induz isomorfismo em homologia, para $p \geq 1$ :

$$
\cdots \longrightarrow \underbrace{H_{p+1}(W)}_{0} \stackrel{\Delta}{\longrightarrow} H_{p}(O \backslash Q) \stackrel{\left(i_{1 \#,-i_{1}}\right)}{\longrightarrow} H_{p}(W \backslash Q) \oplus \underbrace{H_{p}(O)}_{0} \stackrel{k_{1 \#}+k_{2} \#}{\longrightarrow} \underbrace{H_{p}(W)}_{0} \longrightarrow \cdots
$$

Como $O \backslash Q$ e $W \backslash Q$ são ambos simplesmente conexos, esta inclusão é também uma equivalência de homotopia fraca (vide (HATCHER, 2002), Corollary 4.33, pg. 367).

Proposição 5.2.2. Sejam $W$ uma $n$-variedade contrátil, sem bordo e $O \subseteq W$ aberto homeomorfo a $\mathbb{R}^{n}$, com $n \geq 3$. Então, a inclusão $i: O \hookrightarrow W$ induz equivalência de homotopia fraca em todos os espaços de configurações ordenados e não ordenados.

Demonstração. Vejamos que a aplicação induzida sobre os espaços de configurações

$$
F(i, k): F(O, k) \longrightarrow F(W, k)
$$

é uma equivalência de homotopia fraca, para qualquer $k \geq 1$. A prova será feita por indução sobre $k$. Quando $k=1$, segue do Lema 5.2.1, considerando $Q=\emptyset$. Agora, suponhamos que o resultado seja válido para qualquer $1 \leq l \leq k-1$. Note que pelo Teorema 3.1.25, temos uma aplicação de sequências de fibrações (vide Figura 18),

Figura 18 - Diagrama comutativo

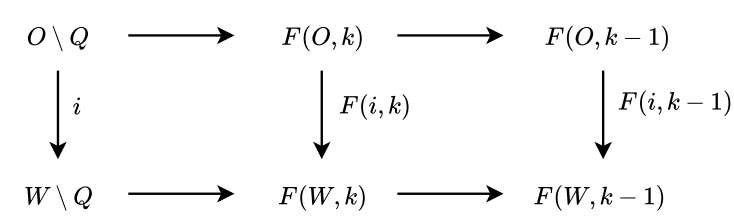

Fonte: Elaborada pelo autor.

onde $Q$ é um subconjunto finito de $O$ com $k-1$ elementos.

O Lema 5.2.1 afirma que a aplicação entre as fibras é uma equivalência de homotopia fraca e, por indução, a aplicação entre os espaços bases é uma equivalência de homotopia fraca, logo concluímos que a aplicação entre os espaços totais

$$
F(i, k): F(O, k) \longrightarrow F(W, k)
$$


Figura 19 - Naturalidade da sequência exata longa da fibração.

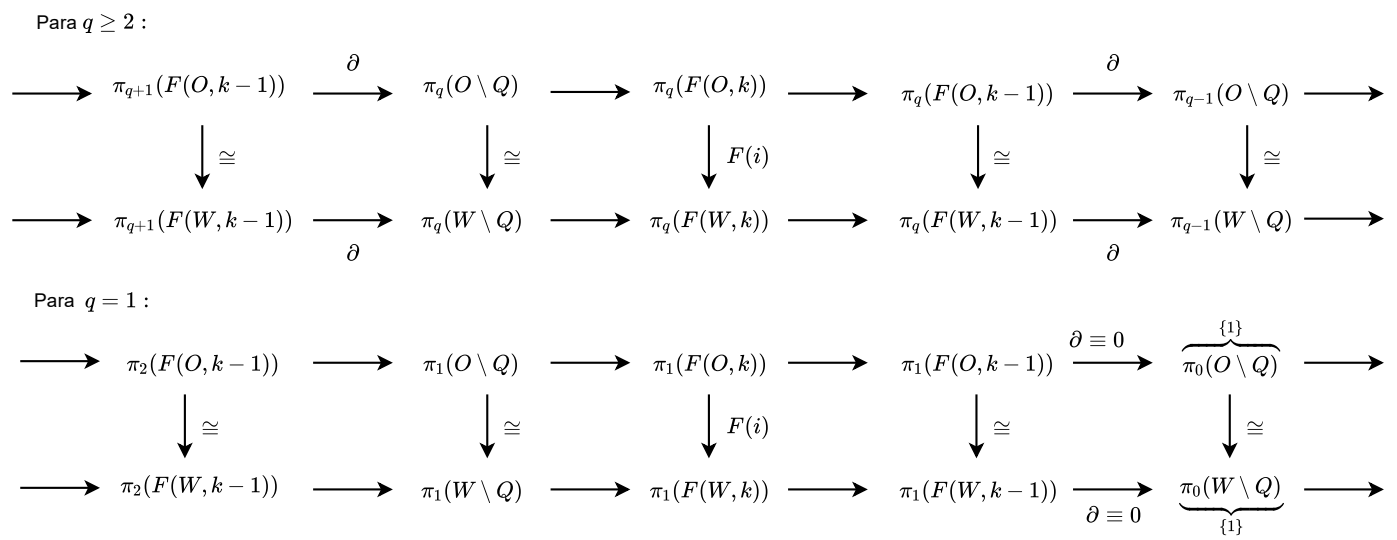

Fonte: Elaborada pelo autor.

é uma equivalência de homotopia fraca, usando a naturalidade da sequência exata longa dos grupos de homotopia da fibração (Teorema 3.1.22) e o Lema dos cinco (Corolário A.0.2), como mostra a Figura 19.

Agora, o resultado para espaços de configurações não ordenados, segue do fato que também temos uma aplicação de sequências de fibrações (vide Figura 20),

Figura 20 - Diagrama comutativo no caso não ordenado

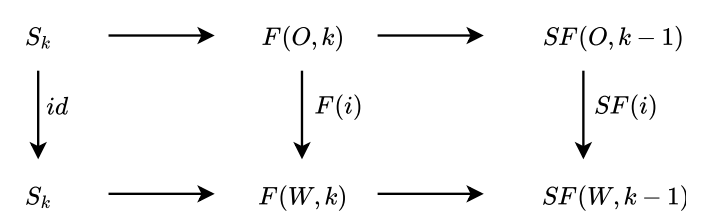

Fonte: Elaborada pelo autor.

a qual, pelo passo precedente, é uma equivalência de homotopia fraca sobre os espaços totais. Concluímos da Proposição B.3.4-(2), que a aplicação sobre os espaços base também é uma equivalência de homotopia fraca.

Corolário 5.2.3. Para $n \geq 3$.

1. Um mergulho $f: V \longrightarrow W$ entre $n$-variedades contráteis, sem bordo, induz uma equivalência de homotopia fraca sobre todos os espaços de configurações ordenados e não ordenados.

2. Se $V$ e $W$ são $n$-variedades contráteis, sem bordo, então existem equivalências de homotopia fraca entre seus espaços de configurações ordenados,

$$
F(V, k) \stackrel{w}{\simeq} F\left(\mathbb{R}^{n}, k\right) \stackrel{w}{\simeq} F(W, k)
$$


e entre seus espaços de configurações não ordenados,

$$
S F(V, k) \stackrel{w}{\simeq} S F\left(\mathbb{R}^{n}, k\right) \stackrel{w}{\simeq} S F(W, k) .
$$

Demonstração. (1) Pelo Teorema da invariância do Domínio (Corolário B.1.3), temos que $f$ é uma aplicação aberta. Seja $O \subseteq V$ um subconjunto aberto homeomorfo ao $\mathbb{R}^{n}$. Então, $\widetilde{O}:=f(O) \subseteq W$ é um subconjunto aberto homeomorfo ao $\mathbb{R}^{n}$, pois $f$ é aberta. Assim, se $i: O \hookrightarrow V$ e $j: \widetilde{O} \hookrightarrow W$ denotam as inclusões naturais, segue da Proposição 5.2.2 que:

$$
\begin{aligned}
& F(i): F(O, k) \longrightarrow F(V, k), S F(i): S F(O, k) \longrightarrow S F(V, k), \\
& F(j): F(\widetilde{O}, k) \longrightarrow F(W, k), S F(j): S F(\widetilde{O}, k) \longrightarrow S F(W, k)
\end{aligned}
$$

são equivalências de homotopia fraca. Dessa forma, considerando os digramas comutativos representados na Figura 21, concluímos que $F(f): F(V, k) \longrightarrow F(W, k)$ é uma equivalência

Figura 21 - Diagramas comutativos

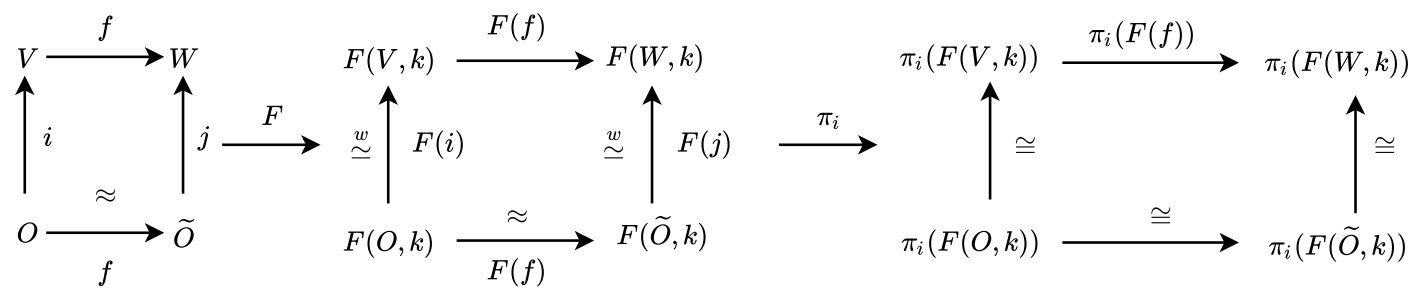

Fonte: Elaborada pelo autor.

de homotopia fraca. O caso não ordenado segue de maneira análoga.

(2) Sejam $O \subseteq V$ e $\widetilde{O} \subseteq W$ subconjuntos abertos homeomorfos ao $\mathbb{R}^{n}$. Então, pelas Proposições 2.1.13-(2) e 5.2.2, concluímos que:

$$
F(V, k) \stackrel{w}{\simeq} F(O, k) \approx F\left(\mathbb{R}^{n}, k\right) \approx F(\widetilde{O}, k) \stackrel{w}{\simeq} F(W, k)
$$

onde $\approx$ significa homeomorfismo. O caso não ordenado segue de maneira análoga.

Observação 5.2.4. Se $V$ for uma $n$-variedade contrátil, sem bordo, com $n \geq 3$, pelo Corolário 5.2.3, seus espaços de configurações, ordenados e não ordenados, possuem o mesmo tipo de homotopia fraca que os correspondentes espaços de configurações do espaço Euclideano $\mathbb{R}^{n}$. Em particular, toda $n$-variedade contrátil, sem bordo possui o mesmo tipo de homotopia fraca que o espaço Euclideano $\mathbb{R}^{n}$, ou seja,

$$
V=F(V, 1) \stackrel{w}{\simeq} F\left(\mathbb{R}^{n}, 1\right)=\mathbb{R}^{n}
$$

Exemplo 5.2.5. A variedade de Whitehead é um exemplo de uma 3-variedade aberta que é contrátil, mas não é homeomorfa ao espaço Euclideano $\mathbb{R}^{3}$. Para detalhes sobre a construção de tal variedade vide (WHITEHEAD, 1935) e (KIRBY, 1989), Chapter XII, Section 4, pg. 81. 
Assim, em particular, temos nosso resultado desejado, como segue.

Teorema 5.2.6. Os espaços de configurações, ordenados e não ordenados, da variedade de Whitehead possuem o mesmo tipo de homotopia fraca que os correspondentes espaços de configurações do espaço Euclideano $\mathbb{R}^{3}$. Isto implica, em particular, que a variedade de Whitehead tem o mesmo tipo de homotopia fraca que o $\mathbb{R}^{3}$, porém a variedade de Whitehead não é homeomorfa a $\mathbb{R}^{3}$. 

CAPÍTULO

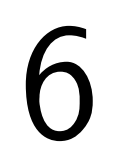

\section{ESPAÇOS DE CONFIGURAÇÕES PARA CERTAS VARIEDADES}

\subsection{Espaços de configurações Euclideanos}

Neste capítulo, nosso objetivo é obter o espaço de configurações Euclideano $F\left(\mathbb{R}^{n}, k\right)$, para todo $k \geq 1$. Tal espaço aparece no problema do $N$-corpo, em teoria de homotopia de espaços de laços iterados (vide (SEGAL, 1973)), em teoria de nós e na teoria de deformação (vide (KONTSEVICH, 1999)).

Proposição 6.1.1. ((WESTERLAND, 2012), Example 1, pg. 279 e (ROTH, 2008), Section 4, pg. 456) O espaço de configurações $F(\mathbb{R}, k)$ possui $k$ ! componentes contráteis e $S F(\mathbb{R}, k)$ é contrátil.

Demonstração. Basta provar para $F(I, k)$, onde $I:=(0,1)$ denota o intervalo aberto em $\mathbb{R}$. De fato, note que a menos da ordenação, $k$-pontos distintos em $I$ são dados por uma sequência crescente de números reais da forma:

$$
0<t_{1}<t_{2}<\ldots<t_{k}<1 .
$$

A coleção de todas tais $k$-uplas $\left(t_{1}, \ldots, t_{k}\right)$ é chamada um $k$-simplexo aberto. Por exemplo, para $k=1,2,3$, teríamos o intervalo $I$, um triângulo aberto e um tetraedro aberto, respectivamente. Assim, $S F(I, k)$ é um simplexo aberto, logo é contrátil e $F(I, k)$ é uma união disjunta de $k$ ! cópias deste simplexo aberto, pois existem exatamente $k$ ! reordenamentos distintos dos $t_{i}$ 's. Explicitamente,

$$
F(I, k)=\bigsqcup_{\sigma \in S_{k}} \Delta_{\sigma} \quad(\text { união disjunta })
$$

onde $\Delta_{\sigma}:=\left\{\left(t_{1}, \ldots, t_{k}\right) \in I^{k}: 0<t_{\sigma(1)}<t_{\sigma(2)}<\ldots<t_{\sigma(k)}<1\right\} \approx \mathbb{R}^{k}$ é um $k$-simplexo aberto, o qual é contrátil, pois é convexo. Na verdade, é homeomorfo ao $k$-espaço Euclideano $\mathbb{R}^{k}$. 
Observação 6.1.2. Uma consequência imediata da Proposição 6.1.1 é que os funtores

$$
F(-, k), F(-, l): \mathfrak{T o p}_{i} \longrightarrow \mathfrak{T o p}_{i}
$$

apresentados na Observação 2.1.11 são isomorfos, ou seja, existe um isomorfismo natural (vide Definição 1.1.9) $\eta: F(-, k) \longrightarrow F(-, l)$ se, e somente se, $k=l$.

Corolário 6.1.3. $F(-, k)$ é isomorfo a $F(-, l)$ se, e somente se, $k=l$.

Demonstração. Se $k=l$, então $F(-, k) \cong F(-, l)$. Reciprocamente, se $F(-, k) \cong F(-, l)$, então $F(\mathbb{R}, k) \cong F(\mathbb{R}, l)$. Pela Proposição 6.1.1, $F(\mathbb{R}, k)$ tem $k$ ! componentes contráteis e $F(\mathbb{R}, l)$ tem $l$ ! componentes contráteis. Assim, $k !=l$ ! e, portanto, $k=l$.

Proposição 6.1.4. Consideremos os grupos de tranças e os grupos de tranças puras de Artin, equipados com a topologia discreta. Então, $F\left(\mathbb{R}^{2}, k\right)$ é o espaço classificante do grupo de tranças puras de Artin sobre $k$ cordas e $S F\left(\mathbb{R}^{2}, k\right)$ é o espaço classificante do grupo de tranças de Artin sobre $k$ cordas, para todo $k \geq 1$ (vide (SALVATORE, 2001), pg. 161 e (FUKS, 1970), Section 1, pg. 143).

Demonstração. Como $F\left(\mathbb{R}^{2}, k\right)$ e $S F\left(\mathbb{R}^{2}, k\right)$ são complexos CW, pelo Corolário 2.1.21, o resultado segue do Exemplo 3.2.3 e do Corolário 1.7.16.

Proposição 6.1.5. ((COHEN, 2010), Example 2.3, pg. 185)

$$
F\left(\mathbb{R}^{n}, 2\right) \simeq \mathbb{S}^{n-1}
$$

Demonstração. Vide Exemplo 2.1.10-(2).

Teorema 6.1.6. ((FADELL; NEUWIRTH, 1962), Section II, Corollary 2.1, pg. 113) Para todo $n>1$ e para todo $i \geq 0$, temos um isomorfismo:

$$
\pi_{i}\left(F\left(\mathbb{R}^{n+1}, k\right)\right) \cong \bigoplus_{r=1}^{k-1} \pi_{i}\left(\bigvee^{r} S^{n}\right)
$$

Demonstração. Pela Proposição 4.1.18, considerando $M=\{p t o\}$, obtemos para $i \geq 2$ :

$$
\pi_{i}\left(F\left(\mathbb{R}^{n+1}, k\right)\right) \cong \bigoplus_{r=0}^{k-1} \pi_{i}\left(\mathbb{R}^{n+1}-Q_{r}\right) .
$$

Note que $\pi_{i}(\mathbb{R}^{n+1}-\underbrace{Q_{0}}_{\emptyset})=0$, além disso, do Corolário B.4.2, $\pi_{i}\left(\mathbb{R}^{n+1}-Q_{r}\right) \cong \pi_{i}\left(\bigvee^{r} \mathbb{S}^{n}\right)$ com $r \geq 1$. Assim, em (6.1.1), obtemos

$$
\pi_{i}\left(F\left(\mathbb{R}^{n+1}, k\right)\right) \cong \bigoplus_{r=1}^{k-1} \pi_{i}\left(\bigvee^{r} S^{n}\right), \quad \forall i \geq 2
$$


Por outro lado, pelo Exemplo 3.1.30, $F\left(\mathbb{R}^{n+1}, k\right)$ é simplesmente conexo, para $n \geq 2$, e como $\bigvee^{r} S^{n}$ também é simplesmente conexo, para $n \geq 2$, concluímos que

$$
\pi_{i}\left(F\left(\mathbb{R}^{n+1}, k\right)\right) \cong 1 \cong \bigoplus_{r=1}^{k-1} \underbrace{\pi_{i}\left(\bigvee^{r} S^{n}\right)}_{1}, \quad i=0,1
$$

Portanto

$$
\pi_{i}\left(F\left(\mathbb{R}^{n+1}, k\right)\right) \cong \bigoplus_{r=1}^{k-1} \pi_{i}\left(\bigvee^{r} S^{n}\right), \quad \forall i \geq 0
$$

Corolário 6.1.7. ((COHEN, 1995), Section I, pg. 20) Os espaços de configurações $F\left(\mathbb{R}^{n+1}, k\right)$ e $S F\left(\mathbb{R}^{n+1}, k\right)$ não são $K(\pi, 1)$-espaços, para todo $n \geq 2$ e para todo $k \geq 2$.

Corolário 6.1.8. Para todo $n>1$ e para todo $k \geq 2$, temos:

$$
\widetilde{H}_{q}\left(F\left(\mathbb{R}^{n+1}, k\right), \mathbb{Z}\right)=0, \quad \forall q<n \quad \text { e } \quad H_{n}\left(F\left(\mathbb{R}^{n+1}, k\right), \mathbb{Z}\right) \cong \bigoplus_{r=1}^{k-1} \pi_{n}\left(\bigvee^{r} S^{n}\right)
$$

onde $\widetilde{H}_{q}$ denota o $q$-ésimo grupo de homologia singular reduzido e $H_{n}$ denota o $n$-ésimo grupo de homologia singular de $F\left(\mathbb{R}^{n+1}, k\right)$.

Demonstração. Sabemos do Exemplo 3.1.30, que $F\left(\mathbb{R}^{n+1}, k\right)$ é $(n-1)$-conexo, para todo $n \geq 2$. Logo, pelo Teorema de Hurewicz (Teorema B.1.9), concluímos que:

$$
\begin{aligned}
& \widetilde{H}_{q}\left(F\left(\mathbb{R}^{n+1}, k\right), \mathbb{Z}\right)=0, \forall q<n \text { e } \\
& H_{n}\left(F\left(\mathbb{R}^{n+1}, k\right), \mathbb{Z}\right) \cong \pi_{n}\left(F\left(\mathbb{R}^{n+1}, k\right)\right) \cong \bigoplus_{r=1}^{k-1} \pi_{n}\left(\bigvee^{r} S^{n}\right)
\end{aligned}
$$

\section{Homologia de alguns espaços de configurações}

Em toda esta seção, denotaremos por $R$ um anel comutativo com identidade e $H_{q}(X ; R)$ denotará o $q$-ésimo $R$-módulo de homologia singular de um espaço topológico $X$ com coeficientes no anel $R$. Em particular, quando $R=\mathbb{Z}$ usaremos em alguns casos simplesmente a notação $H_{q}(X)$.

O resultado a seguir mostra que, para $q$ suficientemente grande, os $R$-módulos de homologia singular $H_{q}(X, R)$ dos espaços de configurações de variedades topológicas conexas são triviais.

Proposição 6.1.9. Seja $M$ uma $m$-variedade topológica conexa. Então:

$$
H_{q+1}(F(M, k) ; R)=0, \quad \forall q \geq m k \text { e } H_{q+1}(S F(M, k) ; R)=0, \quad \forall q \geq m k .
$$


Demonstração. Segue da Observação 1.4 .12 e do Corolário 2.1.19 .

Apresentamos a seguir o cálculo dos grupos de homologia singular de certos espaços de configurações.

Exemplo 6.1.10 (Homologia de $F\left(\mathbb{R}^{n}, 3\right), \quad n \geq 3$ ). Para todo $n \geq 3$, temos:

$$
H_{q}\left(F\left(\mathbb{R}^{n}, 3\right) ; \mathbb{Z}\right)= \begin{cases}\mathbb{Z}, & q=0 ; \\ \mathbb{Z} \oplus \mathbb{Z} \oplus \mathbb{Z}, & q=n-1 ; \\ \mathbb{Z} \oplus \mathbb{Z}, & q=2 n-2 ; \\ 0, & \text { caso contrário. }\end{cases}
$$

De fato, pelo Teorema de Fadell e Neuwirth (Teorema 3.1.25), temos a fibração:

$$
\mathbb{R}^{n}-\left\{p_{1}, p_{2}\right\} \longrightarrow F\left(\mathbb{R}^{n}, 3\right) \longrightarrow F\left(\mathbb{R}^{n}, 2\right) \simeq \mathbb{S}^{n-1}
$$

com fibra $F=\mathbb{R}^{n}-\left\{p_{1}, p_{2}\right\}$. Usando a sequência de Mayer-Vietoris, tem-se:

$$
H_{q}(F)= \begin{cases}\mathbb{Z}, & q=0 \\ \mathbb{Z} \oplus \mathbb{Z}, & q=n-1 \\ 0, & \text { caso contrário. }\end{cases}
$$

Denotemos por $E=F\left(\mathbb{R}^{n}, 3\right)$ o espaço total e por $B=\mathbb{S}^{n-1}$ o espaço base desta fibração, o qual é simplesmente conexo, pois $n \geq 3$. Assim, o $E^{2}$-termo da sequência espectral de Leray-Serre para esta fibração (vide Proposição 1.6.11) é da forma:

$$
E_{p, q}^{2} \cong H_{p}(B) \otimes H_{q}(F)
$$

Logo, os únicos termos não nulos para $E_{*, *}^{2}$ são representados na Figura 22.

Note que todos os diferenciais são nulos, assim, $E_{p, q}^{\infty}=E_{p, q}^{2}$. O espaço total $E$ é conexo por caminhos, $\operatorname{logo} H_{0}(E) \cong \mathbb{Z}$. Para $H_{n-1}$, existe a filtração com $n$-termos:

$$
0=F_{-1} H_{n-1} \subseteq F_{0} H_{n-1} \subseteq \cdots \subseteq F_{n-3} H_{n-1} \subseteq F_{n-2} H_{n-1} \subseteq F_{n-1} H_{n-1}=H_{n-1} .
$$

Assim,

$$
H_{n-1} / F_{n-2} H_{n-1}=F_{n-1} H_{n-1} / F_{n-2} H_{n-1} \cong E_{n-1,0}^{\infty} \cong \mathbb{Z} .
$$

Como $\mathbb{Z}$ é um $\mathbb{Z}$-módulo livre, isso implica que

$$
H_{n-1} \cong F_{n-2} H_{n-1} \oplus \mathbb{Z}
$$

Por outro lado,

$$
F_{n-2} H_{n-1} / F_{n-3} H_{n-1} \cong E_{n-2,1}^{\infty} \cong 0 \text {, então } F_{n-2} H_{n-1}=F_{n-3} H_{n-1} \text {. }
$$


Figura $22-E_{*, *}^{2}$

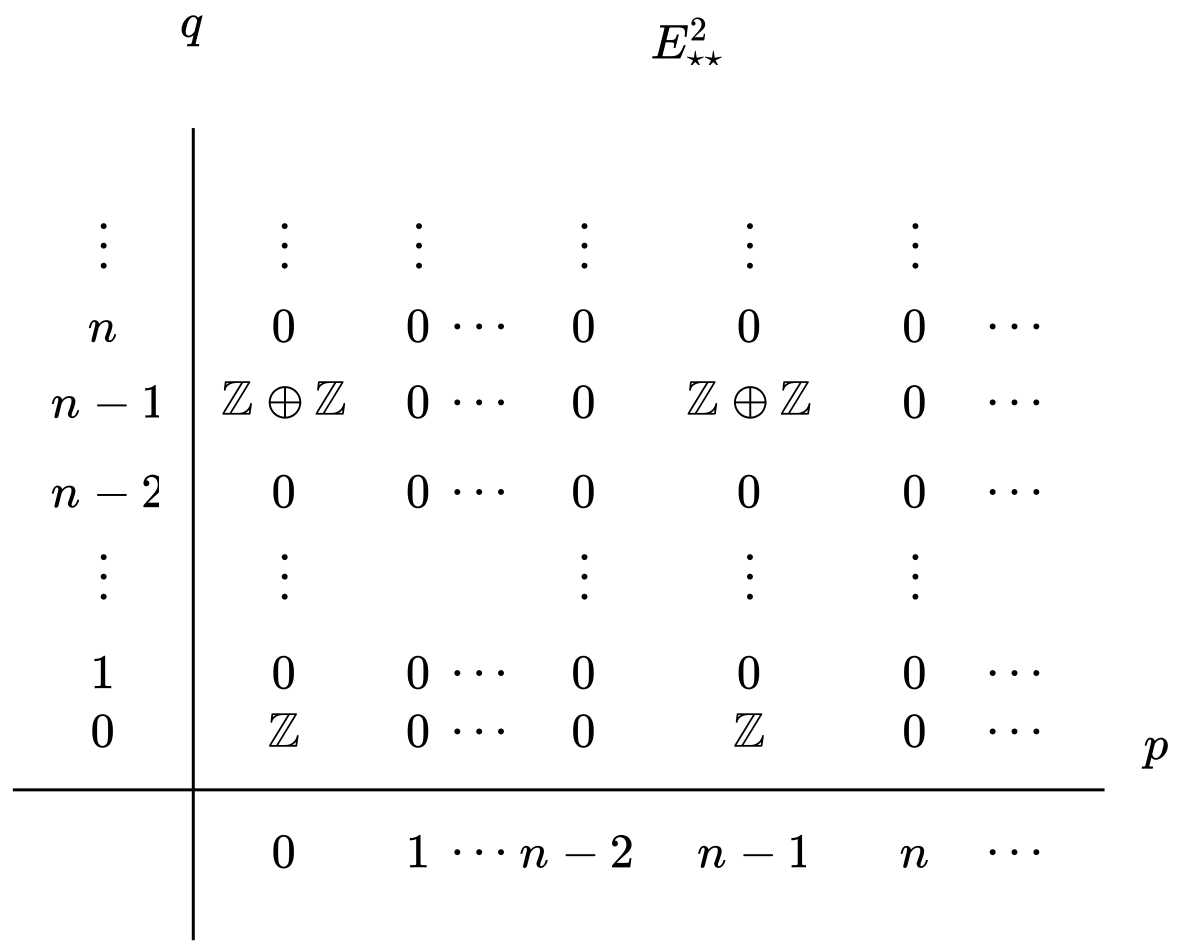

Fonte: Elaborada pelo autor.

Continuando este processo, obtemos o quociente

$$
F_{0} H_{n-1}=F_{0} H_{n-1} / F_{-1} H_{n-1} \cong E_{0, n-1}^{\infty} \cong \mathbb{Z} \oplus \mathbb{Z}
$$

Assim,

$$
F_{n-2} H_{n-1}=F_{n-3} H_{n-1}=\cdots=F_{1} H_{n-1}=F_{0} H_{n-1} \cong \mathbb{Z} \oplus \mathbb{Z}
$$

Portanto,

$$
H_{n-1}(E) \cong \mathbb{Z} \oplus \mathbb{Z} \oplus \mathbb{Z}
$$

De maneira análoga, conclui-se que

$$
H_{2 n-2}(E) \cong \mathbb{Z} \oplus \mathbb{Z}
$$

Observação 6.1.11. Do exemplo 6.1.10, temos os números de Betti de $F\left(\mathbb{R}^{n}, 3\right), n \geq 3$ :

$$
\beta_{q}:=\beta_{q}\left(F\left(\mathbb{R}^{n}, 3\right)\right):=\operatorname{rank}\left(H_{q}\left(F\left(\mathbb{R}^{n}, 3\right)\right)\right)= \begin{cases}1, & q=0 \\ 3, & q=n-1 \\ 2, & q=2 n-2 \\ 0, & \text { caso contrário. }\end{cases}
$$


Logo, a Característica de Euler de $F\left(\mathbb{R}^{n}, 3\right)$ tem a forma:

$$
\chi\left(F\left(\mathbb{R}^{n}, 3\right)\right):=\sum_{q \geq 0}(-1)^{q} \beta_{q}=3\left(1+(-1)^{n-1}\right),
$$

e seu Polinômio de Poincaré tem a forma:

$$
P\left(F\left(\mathbb{R}^{n}, 3\right)\right)(t):=\sum_{q \geq 0} \beta_{q} t^{q}=1+3 t^{n-1}+2 t^{2 n-2} .
$$

Exemplo 6.1.12 (Homologia de $F\left(\mathbb{R}^{n}, 4\right), n \geq 3$ ). Para todo $n \geq 3$, temos:

$$
H_{q}\left(F\left(\mathbb{R}^{n}, 4\right) ; \mathbb{Z}\right)= \begin{cases}\mathbb{Z}, & q=0 \\ \mathbb{Z}^{\oplus 6}, & q=n-1 \\ \mathbb{Z}^{\oplus 11}, & q=2 n-2 \\ \mathbb{Z}^{\oplus 6}, & q=3 n-3 ; \\ 0, & \text { caso contrário. }\end{cases}
$$

Pelo teorema de Fadell e Neuwirth (Teorema 3.1.25), temos a fibração:

$$
\mathbb{R}^{n}-\left\{p_{1}, p_{2}, p_{2}\right\} \longrightarrow F\left(\mathbb{R}^{n}, 4\right) \longrightarrow F\left(\mathbb{R}^{n}, 3\right)
$$

com fibra $F=\mathbb{R}^{n}-\left\{p_{1}, p_{2}, p_{3}\right\}$. Usando a sequência de Mayer-Vietoris, tem-se:

$$
H_{q}(F)= \begin{cases}\mathbb{Z}, & q=0 ; \\ \mathbb{Z} \oplus \mathbb{Z} \oplus \mathbb{Z}, & q=n-1 ; \\ 0, & \text { caso contrário. }\end{cases}
$$

Denotemos por $E=F\left(\mathbb{R}^{n}, 4\right)$ o espaço total e por $B=F\left(\mathbb{R}^{n}, 3\right)$ o espaço base desta fibração, o qual é simplesmente conexo, pois $n \geq 3$ (Teorema 6.1.6). Assim, o $E^{2}$-termo da sequência espectral de Leray-Serre para esta fibração (vide Proposição 1.6.11), é dado por:

$$
E_{p, q}^{2} \cong H_{p}(B) \otimes H_{q}(F)
$$

Logo, os únicos termos não nulos para $E_{*, *}^{2}$ são representados na Figura 23.

Note que todos os diferenciais são nulos, assim, $E_{p, q}^{\infty}=E_{p, q}^{2}$. O espaço total $E$ é conexo por caminhos, $\log 0 H_{0}(E) \cong \mathbb{Z}$. Usando a mesma estratégia do Exemplo 6.1.10, concluímos:

$$
\begin{aligned}
H_{n-1}(E) & \cong \mathbb{Z}^{\oplus 3} \oplus \mathbb{Z}^{\oplus 3}=\mathbb{Z}^{\oplus 6} \\
H_{2 n-2}(E) & \cong \mathbb{Z}^{\oplus 9} \oplus \mathbb{Z}^{\oplus 2}=\mathbb{Z}^{\oplus 11} \\
H_{3 n-3}(E) & \cong \mathbb{Z}^{\oplus 6} .
\end{aligned}
$$

Observação 6.1.13. Do Exemplo 6.1.12, temos os números de Betti para $F\left(\mathbb{R}^{n}, 4\right), n \geq 3$ :

$$
\beta_{q}:=\beta_{q}\left(F\left(\mathbb{R}^{n}, 4\right)\right):=\operatorname{rank}\left(H_{q}\left(F\left(\mathbb{R}^{n}, 4\right)\right)\right)= \begin{cases}1, & q=0 \\ 6, & q=n-1 ; \\ 11, & q=2 n-2 ; \\ 6, & q=3 n-3 ; \\ 0, & \text { caso contrário. }\end{cases}
$$


Figura 23 - Sequência espectral do espaço de configurações $F\left(\mathbb{R}^{n}, 4\right)$.

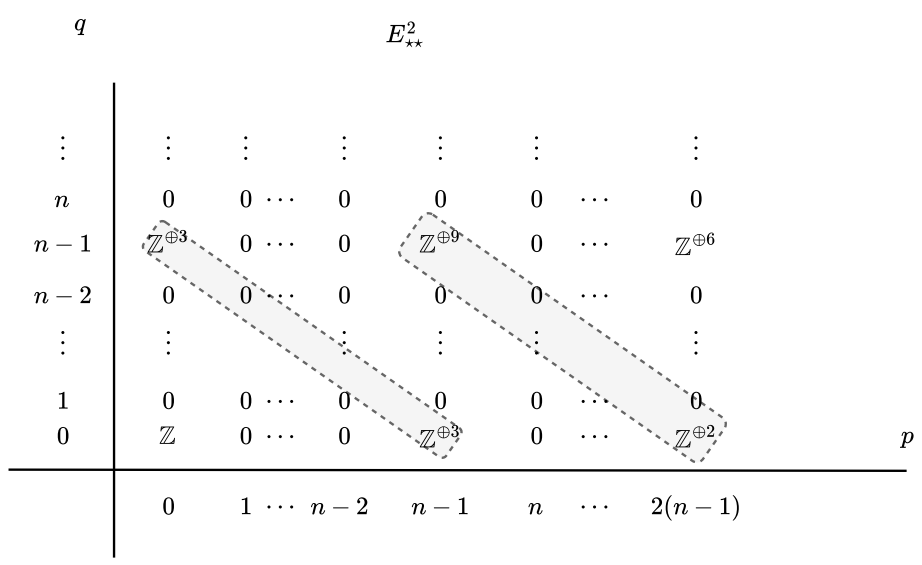

Fonte: Elaborada pelo autor.

Logo, a Característica de Euler de $F\left(\mathbb{R}^{n}, 4\right)$ tem a forma:

$\chi\left(F\left(\mathbb{R}^{n}, 4\right)\right):=\sum_{q \geq 0}(-1)^{q} \beta_{q}=1+6(-1)^{n-1}+11+6(-1)^{3 n-3}=6\left(2+(-1)^{n-1}+(-1)^{3 n-3}\right)$,

e o seu Polinômio de Poincaré tem a forma:

$$
P\left(F\left(\mathbb{R}^{n}, 3\right)\right)(t):=\sum_{q \geq 0} \beta_{q} t^{q}=1+6 t^{n-1}+11 t^{2 n-2}+6 t^{3 n-3}
$$

Exemplo 6.1.14 (Homologia de $F\left(\mathbb{C P}^{n}, 2\right), n \geq 1$ ). Para todo $n \geq 1$, temos:

$$
H_{q}\left(F\left(\mathbb{C P}^{n}, 2\right) ; \mathbb{Z}\right)= \begin{cases}\mathbb{Z}^{\oplus\left(\frac{q}{2}+1\right)}, & q=0,2,4, \ldots, 2(n-1) ; \\ \mathbb{Z}^{\oplus\left(2 n-\frac{q}{2}\right)}, & q=2 n, 2 n+2,2 n+4, \ldots, 2 n+2(n-1) ; \\ 0, & \text { caso contrário. }\end{cases}
$$

O espaço projetivo complexo $\mathbb{C P}^{n}$ é uma variedade compacta sem bordo. Pelo teorema de Fadell e Neuwirth (Teorema 3.1.25), temos a fibração

$$
\mathbb{C} \mathbb{P}^{n-1} \simeq \mathbb{C P}^{n}-\{\star\} \longrightarrow F\left(\mathbb{C P}^{n}, 2\right) \longrightarrow \mathbb{C P}^{n}
$$

com fibra $F=\mathbb{C P}^{n-1}$, espaço total $E=F\left(\mathbb{C P}^{n}, 2\right)$ e espaço base $B=\mathbb{C P}^{n}$, o qual é simplesmente conexo (lembremos do fibrado $\mathbb{S}^{1} \hookrightarrow \mathbb{S}^{2 n+1} \longrightarrow \mathbb{C P}^{n}$ ). Assim o $E^{2}$-termo da sequência espectral de Leray-Serre para esta fibração (vide Proposição 1.6.11) tem a forma:

$$
E_{p, q}^{2} \cong H_{p}(B) \otimes H_{q}(F) \cong H_{p}\left(\mathbb{C P}^{n}\right) \otimes H_{q}\left(\mathbb{C P}^{n-1}\right)
$$

Logo, os únicos termos não nulos para $E_{*, *}^{2}$ são representados na Figura 24.

Note que todos seus diferenciais são nulos, assim, $E_{p, q}^{\infty}=E_{p, q}^{2}$. O resultado segue usando a mesma estratégia dos Exemplos 6.1.10 e 6.1.12. 
Figura 24 - Sequência espectral do espaço de configurações $F\left(\mathbb{C P}^{n}, 2\right)$.

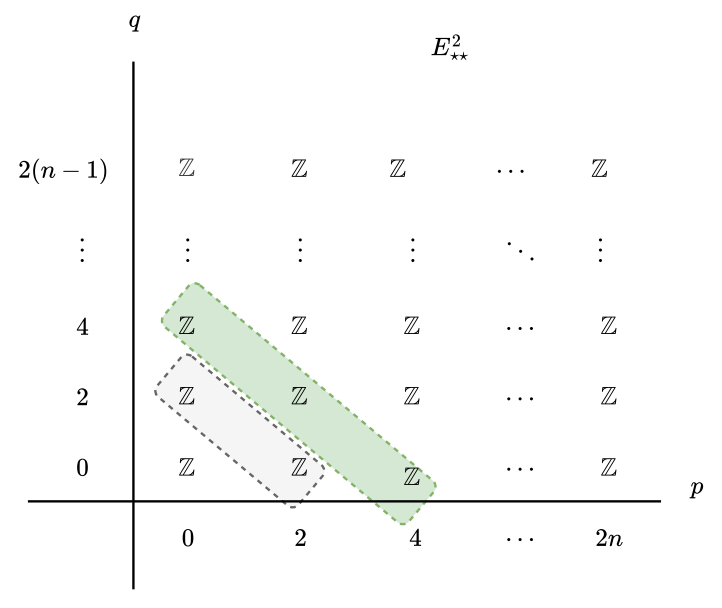

Fonte: Elaborada pelo autor.

Observação 6.1.15. Do Exemplo 6.1.14, os números de Betti para $F\left(\mathbb{C P}^{n}, 2\right), n \geq 1$ são:

$$
\begin{aligned}
\beta_{q} & :=\beta_{q}\left(F\left(\mathbb{C P}^{n}, 2\right)\right) \\
& :=\operatorname{rank}\left(H_{q}\left(F\left(\mathbb{C P}^{n}, 2\right)\right)\right) \\
& = \begin{cases}j+1, & q=2 j, \quad j=0,1,2, \ldots, n-1 ; \\
2 n-j, & q=2 j, \quad j=n, n+1, n+2 \ldots, n+(n-1) ; \\
0, & \text { caso contrário. }\end{cases}
\end{aligned}
$$

Logo, a característica de Euler de $F\left(\mathbb{C P}^{n}, 2\right)$ tem a forma:

$\chi\left(F\left(\mathbb{C P}^{n}, 2\right)\right):=\sum_{q \geq 0}(-1)^{q} \beta_{q}=\sum_{j=0}^{n-1}(j+1)+\sum_{j=n}^{n+n-1}(2 n-j)=\sum_{j=0}^{n-1}(j+1)+\sum_{j=0}^{n-1}(n-j)=n+n^{2}$,

e seu Polinômio de Poincaré tem a forma:

$$
P\left(F\left(\mathbb{C P}^{n}, 2\right)\right)(t):=\sum_{q \geq 0} \beta_{q} t^{q}=\sum_{j=0}^{n-1}(j+1) t^{2 j}+\sum_{j=n}^{n+(n-1)}(2 n-j) t^{2 j} .
$$

Uma das razões importantes de se conhecer os grupos de homologia de um espaço topológico é dada na seguinte aplicação.

Proposição 6.1.16. ((HATCHER, 2002), Proposition 4C.1, pg. 439) Um CW complexo simplesmente conexo, cujos grupos de homologia são finitamente gerados, tem o mesmo tipo de homotopia de um CW complexo tendo um número mínimo de células consistentes com seus grupos de homologia, isto é, uma $n$-célula para cada somando $\mathbb{Z}$ de $H_{n}$, e um par de células de dimensões $n$ e $n+1$, para cada somando $\mathbb{Z}_{k}$ de $H_{n}$. 
Exemplo 6.1.17. Para o caso da $n$-esfera $\mathbb{S}^{n}(n \geq 2)$, seus grupos de homologia são:

$$
H_{q}\left(\mathbb{S}^{n} ; \mathbb{Z}\right) \cong \begin{cases}\mathbb{Z}, & q=0, n \\ 0, & q \neq 0, n .\end{cases}
$$

Então, pela Proposição 6.1.16, a esfera $\mathbb{S}^{n}$ tem o mesmo tipo de homotopia de um CW complexo formado por uma 0 -célula e uma $n$-célula.

Exemplo 6.1.18. Do Exemplo 6.1.10, obtemos que o espaço de configurações $F\left(\mathbb{R}^{n}, 3\right)$ $(n \geq 3)$ tem o tipo de homotopia de um CW complexo formado por uma 0-célula, três $(n-1)$-células e duas $2(n-1)$-células. Assim, $F\left(\mathbb{R}^{n}, 3\right)$ tem o mesmo tipo de homotopia de um 2(n-1)-dimensional CW complexo finito. Analogamente, do Exemplo 6.1.12, obtemos que o espaço de configurações $F\left(\mathbb{R}^{n}, 4\right)(n \geq 3)$ tem o mesmo tipo de homotopia de um 3(n-1)-dimensional CW complexo finito.

Exemplo 6.1.19. Do Exemplo 6.1.14, obtemos que o espaço de configurações $F\left(\mathbb{C P}^{n}, 2\right)$ tem o mesmo tipo de homotopia de um $2(2 n-1)$-dimensional CW complexo finito. $F\left(\mathbb{C P}^{n}, 2\right)$ é simplesmente conexo, pelo Exemplo 3.1.32.

\subsection{Espaços de configurações da esfera $\mathbb{S}^{n}$}

Nesta seção, apresentamos algumas propriedades elementares dos espaços de configurações da $n$-esfera $F\left(\mathbb{S}^{n}, k\right)$, para todo $n \geq 1$.

Usando a Proposição 6.1.1, obtemos o seguinte resultado.

Proposição 6.2.1. O espaço de configurações da 1-esfera $S^{1}, F\left(\mathbb{S}^{1}, k+1\right)$ é homeomorfo a $\mathbb{S}^{1} \times F(I, k)$, onde $I=(0,1)$. Assim, $F\left(\mathbb{S}^{1}, k+1\right)$ possui $k$ ! componentes conexas por caminhos, as quais tem o mesmo tipo de homotopia de $\mathbb{S}^{1}$.

Demonstração. Sabemos que $\mathbb{S}^{1}$ é um grupo topológico com elemento identidade 1. Assim, do Exemplo 2.1.10-(5),

$$
F\left(\mathbb{S}^{1}, k+1\right) \approx \mathbb{S}^{1} \times F\left(\mathbb{S}^{1}-\{1\}, k\right) \approx \mathbb{S}^{1} \times F(\mathbb{R}, k)
$$

onde $\approx$ significa homeomorfismo. Pela Proposição 6.1.1, concluímos que $F\left(\mathbb{S}^{1}, k+1\right)$ é o produto da 1-esfera $\mathbb{S}^{1}$ com uma união disjunta de $k$ ! simplexos abertos.

Proposição 6.2.2. ((COHEN, 2010), Example 2.4, pg. 186) O espaço de configurações $F\left(\mathbb{S}^{n}, 2\right)$ tem o mesmo tipo de homotopia da $n$-esfera $\mathbb{S}^{n}$.

Demonstração. Vide Example 2.1.10-(6).

Proposição 6.2.3. O espaço de configurações $F\left(\mathbb{S}^{n}, k\right)$ é $(n-2)$-conexo, para todo $n \geq 2$ 
Demonstração. Consideremos a fibração

$$
F\left(\mathbb{R}^{n}, k-1\right) \approx F\left(\mathbb{S}^{n}-\{\star\}, k-1\right) \longrightarrow F\left(\mathbb{S}^{n}, k\right) \stackrel{\pi_{k, 1}}{\longrightarrow} \mathbb{S}^{n}
$$

com fibra $F\left(\mathbb{R}^{n}, k-1\right)$, a qual é $(n-2)$-conexa (vide Teorema 6.1.6) e espaço base $\mathbb{S}^{n}$, o qual é $(n-1)$-conexo. Então, o espaço total $F\left(\mathbb{S}^{n}, k\right)$ é $(n-2)$-conexo. Outra prova foi apresentada no Exemplo 3.1.31.

Observação 6.2.4. Como um caso particular da Proposição 6.2.3, temos que $F\left(\mathbb{S}^{n}, k\right)$ é simplesmente conexo, $\forall n \geq 3$. Logo, os grupos de tranças puras e os grupos de tranças de Artin para a $n$-esfera $\mathbb{S}^{n}, \operatorname{com} n \geq 3$ são:

$$
P B_{k}\left(\mathbb{S}^{n}\right)=\pi_{1}\left(F\left(\mathbb{S}^{n}, k\right)\right)=0 \quad \text { e } \quad B_{k}\left(\mathbb{S}^{n}\right)=\pi_{1}\left(S F\left(\mathbb{S}^{n}, k\right)\right) \cong S_{k}, \quad \forall k .
$$


APÊNDICE

\section{A}

FATOS ALGÉBRICOS

Neste apêndice, apresentamos dois resultados básicos de Álgebra Homológica: o Lema dos Quatro e o Lema dos Cinco. Estaremos considerando os objetos e os morfismos na categoria Grp (grupos e homomorfismos de grupos).

Teorema A.0.1 (Lema dos Quatro). Suponhamos que nos diagramas das Figuras 25 e 26 as duas linhas sejam sequências exatas e os três quadrados sejam comutativos.

Figura 25 - Lema dos quatro

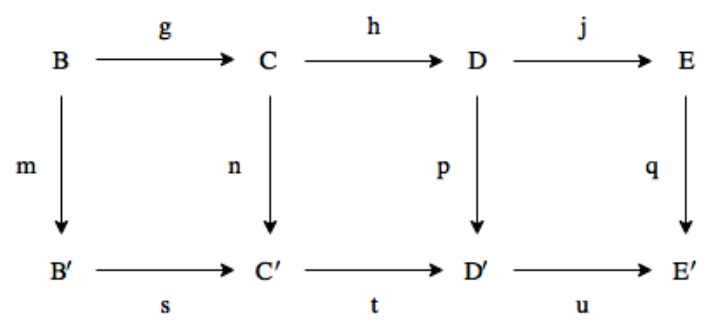

Fonte: Elaborada pelo autor.

(1) Se $m$ e $p$ são epimorfismos e se $q$ for um monomorfismo, então, $n$ é um epimorfismo.

Figura 26 - Lema dos quatro

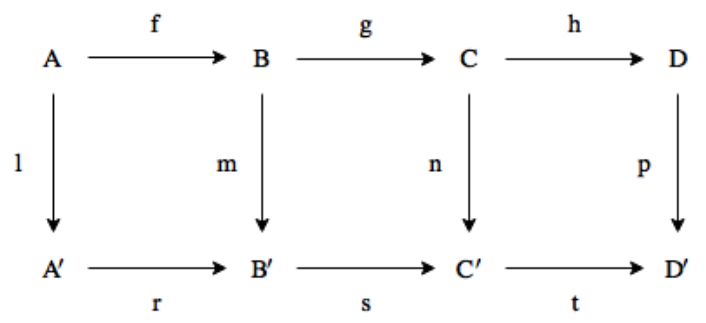

Fonte: Elaborada pelo autor.

(2) Se $m$ e $p$ são monomorfismos e se $l$ for um epimorfismo, então $n$ é um monomorfismo. 
Corolário A.0.2 (Lema dos Cinco). ((SELICK, 2008), Chapter 4, Section 1, Lemma 4.1.6, pg. 22) Suponhamos que no diagrama da Figura 27, as duas linhas sejam sequências exatas e os quatro quadrados sejam comutativos.

Figura 27 - Lema dos cinco

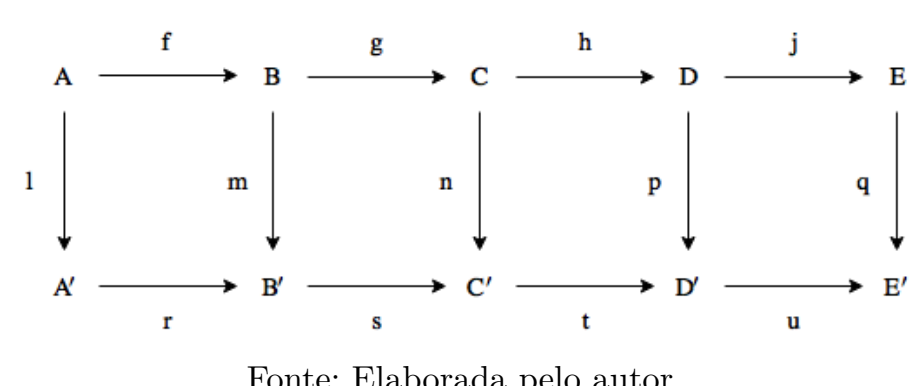

Fonte: Elaborada pelo autor.

Se $m$ e $p$ são isomorfismos, $l$ é um epimorfismo e $q$ é um monomorfismo, então $n$ é um isomorfismo. 
APÊNDICE

B

.

FATOS TOPOLÓGICOS

Neste apêndice, destacamos teoremas importantes que serão usados neste trabalho. Dentre eles estão: o Teorema da Invariância do Domínio, a Sequência de Mayer-Vietoris e o Teorema de Hurewicz. Além disso, apresentamos uma demonstração de que toda variedade topológica conexa $M$, com $\operatorname{dim} M \geq 2$, é $m$-homogênea, para todo $m$. Este resultado foi afirmado por Fadell e Neuwirth em (FADELL; NEUWIRTH, 1962), Section II, pg. 111 (vide Seção B.2).

\section{B.1 Ferramentas topológicas}

Teorema B.1.1 (Teorema da Invariância do Domínio). ((DIECK, 2008), pg. 251, Theorem 10.3.7) Sejam $U$ um subconjunto aberto de $\mathbb{R}^{n}$ e $f: U \longrightarrow \mathbb{R}^{n}$ uma aplicação contínua e injetora. Então, $f$ é uma aplicação aberta e $f: U \longrightarrow f(U)$ é um homeomorfismo.

Observação B.1.2. Mais geralmente, é possível mostrar que todo mergulho entre variedades topológicas de mesma dimensão é uma aplicação aberta, como mostra o resultado a seguir.

Corolário B.1.3. Sejam $M$ e $N$ n-variedades topológicas sem bordo e $f: M \longrightarrow N$ uma aplicação contínua e injetora. Então $f$, é uma aplicação aberta.

Demonstração. Seja $f: M \longrightarrow N$ uma aplicação contínua e injetora entre $n$-variedades topológicas $M$ e $N$. Observemos primeiramente que se $\mathcal{V}=\bigcup\{V\}$ é uma cobertura aberta para $N$, então para qualquer subconjunto $V \in \mathcal{V}$ :

$$
f: M \longrightarrow N \text { é aberta }\left.\Longleftrightarrow f\right|_{f^{-1}(V)}: f^{-1}(V) \longrightarrow V \text { é aberta . }
$$

De fato, dado qualquer subconjunto $W \subset M$, sabemos que

$$
f(W)=\bigcup_{V \in \mathcal{V}} f\left(W \cap f^{-1}(V)\right) .
$$


Em particular, se $W$ for aberto em $M$, então $f(W)$ será aberto em $N$.

Analogamente, dada uma cobertura aberta $\mathcal{U}=\bigcup\{U\}$ para $M$, então para qualquer subconjunto $U \in \mathcal{U}$ :

$$
f: M \longrightarrow N \quad \text { é aberta }\left.\Longleftrightarrow f\right|_{U}: U \longrightarrow N \quad \text { é aberta }
$$

De fato, dado qualquer subconjunto $W \subset M$, sabemos que

$$
f(W)=\bigcup_{U \in \mathcal{U}} f(W \cap U)
$$

Em particular, se $W$ for aberto em $M$, então $f(W)$ será aberto em $N$.

Assim, nosso problema se reduz a mostrar que para quaisquer subconjuntos abertos $U \subset M$ e $V \subset N$, ambos homeomorfos ao $\mathbb{R}^{n}$, a aplicação $f: U \longrightarrow V$ é aberta. Isso segue aplicando-se o Teorema B.1.1 para a composição:

$$
\mathbb{R}^{n} \stackrel{\varphi}{\longrightarrow} U \stackrel{f}{\longrightarrow} V \stackrel{\psi}{\longrightarrow} \mathbb{R}^{n}
$$

onde $\varphi$ e $\psi$ são homeomorfismos.

Uma técnica eficiente para se calcular o grupo fundamental de espaços com ponto base é dada pelo famoso Teorema de Seifert-van Kampen, como segue.

Teorema B.1.4 (Teorema de Seifert-van Kampen). ((LEE, 2010), Theorem 10.1, pg. 252) Seja $\left(X, x_{0}\right)$ um espaço com ponto base tal que $X=U \cup V$ é uma cobertura aberta, com $U, V$ e $U \cap V$ conexos por caminhos e tais que $x_{0} \in U \cap V$. Então,

$$
\pi_{1}\left(X, x_{0}\right) \cong \frac{\pi_{1}\left(U, x_{0}\right) * \pi_{1}\left(V, x_{0}\right)}{N}
$$

onde $N$ é o fecho normal no produto livre $\pi_{1}\left(U, x_{0}\right) * \pi_{1}\left(V, x_{0}\right)$ gerado pela palavra:

$$
i_{1 *}([\alpha])\left(i_{2 *}([\alpha])\right)^{-1}, \quad \forall[\alpha] \in \pi_{1}\left(U \cap V, x_{0}\right)
$$

onde $i_{1}: U \cap V \hookrightarrow U$ e $i_{2}: U \cap V \hookrightarrow V$ são as inclusões naturais.

Exemplo B.1.5. Seja $M$ é um $m$-variedade conexa, com $m \geq 3$. Então, para todo $k \geq 1$ :

$$
\pi_{1}\left(M-Q_{k}\right) \cong \pi_{1}(M)
$$

onde $Q_{k}$ é um subconjunto finito de $M$ contendo $k$-elementos.

Demonstração. A prova será feita por indução sobre $k$. Para $k=1$, usaremos o Teorema de Seifert-van Kampen. Consideremos os subconjuntos $U:=M-Q_{1}$, onde $Q_{1}=\{q\}$ e $V:=U_{q}$ uma vizinhança aberta para $q$ homeomorfa ao $\mathbb{R}^{m}$. Observemos que:

$$
U \cup V=M \text { e } U \cap V=U_{q}-\{q\} \approx R^{m}-\{0\} \simeq \mathbb{S}^{m-1}
$$


onde $\approx$ significa homeomorfismo e $\simeq$ tipo de homotopia. Temos que $M=U \cup V$ é uma cobertura aberta, com $U, V$ e $U \cap V$ conexos por caminhos e $x_{0} \in U \cap V$. Além disso:

$$
\begin{aligned}
\pi_{1}\left(U, x_{0}\right) & =\pi_{1}\left(M-Q_{1}, x_{0}\right) \\
\pi_{1}\left(V, x_{0}\right) & \cong \pi_{1}\left(\mathbb{R}^{m}, \varphi\left(x_{0}\right)\right)=0, \\
\pi_{1}\left(U \cap V, x_{0}\right) & \cong \pi_{1}\left(\mathbb{S}^{m-1}, y_{0}\right)=0, \text { se } m-1 \geq 2,
\end{aligned}
$$

onde $\varphi: V \rightarrow \mathbb{R}^{m}$ é um homeomorfismo. Segue do Teorema B.1.4 que $\pi_{1}\left(M-Q_{1}\right) \cong \pi_{1}(M)$. Suponhamos que o resultado seja válido para quaisquer $1 \leq l \leq k-1$. Consideremos os subconjuntos finitos de $M, Q_{k}=\left\{q_{1}, \ldots q_{k-1}, q_{k}\right\}$ e $Q_{k-1}=\left\{q_{1}, \ldots q_{k-1}\right\}$. Note que $M-Q_{k}=\left(M-Q_{k-1}\right)-\left\{q_{k}\right\}$. Pela Proposição 2.1.24, $M-Q_{k-1}$ é uma $m$-variedade conexa. Então, pelo passo inicial da indução, obtemos:

$$
\pi_{1}\left(M-Q_{k}\right) \cong \pi_{1}\left(M-Q_{k-1}\right)
$$

Agora, usando a hipótese de indução, concluímos que: $\pi_{1}\left(M-Q_{k}\right) \cong \pi_{1}(M)$.

Uma técnica essencial para se calcular os grupos de homologia de certos espaços topológicos é a existência de uma sequência exata longa em homologia, chamada a sequência de MayerVietoris, como segue.

Teorema B.1.6 (Sequência de Mayer-Vietoris). ((VICK, 1994), Chapter 1, pg. 21)

Sejam $X$ um espaço topológico e $A \cup B$ uma cobertura aberta de $X$, com $A \cap B \neq \emptyset$. Então, existe uma sequência exata longa de grupos de homologia (com coeficientes em $\mathbb{Z}$ ),

$$
\cdots \longrightarrow H_{p}(A \cap B) \stackrel{\left(i_{1 *},-i_{2 *}\right)}{\longrightarrow} H_{p}(A) \oplus H_{p}(B) \stackrel{k_{1_{*}+k_{2 *}}}{\longrightarrow} H_{p}(X) \stackrel{\Delta}{\longrightarrow} H_{p-1}(A \cap B) \longrightarrow \cdots
$$

na qual $i_{1}: A \cap B \hookrightarrow A, i_{2}: A \cap B \hookrightarrow B, k_{1}: A \hookrightarrow X$ e $k_{2}: B \hookrightarrow X$ são inclusões. $\mathrm{O}$ homomorfismo $\Delta: H_{p}(X) \longrightarrow H_{p-1}(A \cap B)$ é chamado o homomorfismo conectante.

A seguir, definimos o homomorfismo de Hurewicz, o qual conecta os grupos de homotopia e os grupos de homologia de um espaço topológico.

Definição B.1.7. ((HATCHER, 2002), Chapter 4, Section 4.2, pg. 370)

Para qualquer natural $q \geq 1$, existe um homomorfismo:

$$
h: \pi_{q}\left(X, x_{0}\right) \longrightarrow H_{q}(X ; \mathbb{Z}), \quad[\alpha] \mapsto h([\alpha]):=\alpha_{*}(\iota),
$$

chamado o homomorfismo de Hurewicz, onde $\alpha_{*}: H_{q}\left(\mathbb{S}^{q} ; \mathbb{Z}\right) \longrightarrow H_{q}(X ; \mathbb{Z})$ é o homomorfismo induzido pela função contínua $\alpha:\left(\mathbb{S}^{q}, s_{0}\right) \longrightarrow\left(X, x_{0}\right)$ e $\iota \in H_{q}\left(\mathbb{S}^{q} ; \mathbb{Z}\right) \cong \mathbb{Z}$ é um gerador. 
Observação B.1.8. ((HATCHER, 2002), Chapter 4, Section 4.2, pg. 370)

O homomorfismo de Hurewicz satisfaz a propriedade da naturalidade, ou seja, uma função contínua $f:\left(X, x_{0}\right) \longrightarrow\left(Y, y_{0}\right)$ induz um diagrama comutativo como na Figura 28.

Figura 28 - Naturalidade do homomorfismo de Hurewicz.

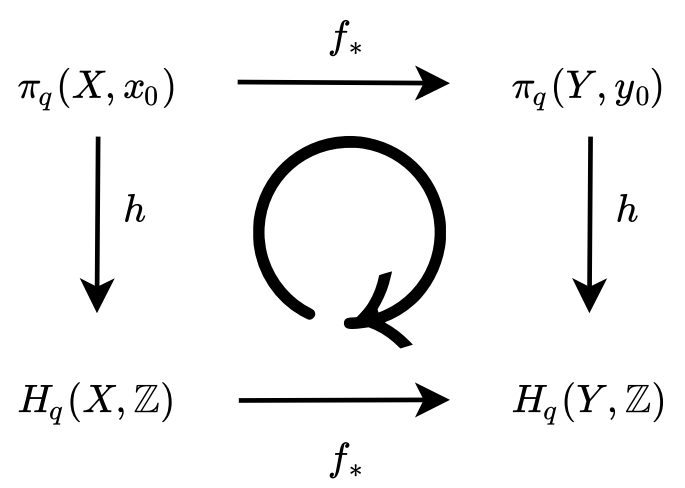

Fonte: Elaborada pelo autor.

Teorema B.1.9 (Teorema de Hurewicz). (HATCHER, 2002), Chapter 4, Section 4.2, Theorem 4.37, pg. 371 e (MASSEY, 1991), Chapter VIII, Section 7, Theorem 7.1, pg. 219)

(a) Se $X$ é $(n-1)$-conexo, com $n \geq 2$, então, o homomorfismo de Hurewicz

$$
h: \pi_{q}\left(X, x_{0}\right) \longrightarrow H_{q}(X ; \mathbb{Z})
$$

é um isomorfismo, para todo $q \leq n$. Além disso,

$$
h: \pi_{n+1}\left(X, x_{0}\right) \longrightarrow H_{n+1}(X ; \mathbb{Z})
$$

é um epimorfismo.

(b) Se $X$ é 0-conexo, então $\pi_{1}\left(X, x_{0}\right) /\left[\pi_{1}\left(X, x_{0}\right), \pi_{1}\left(X, x_{0}\right)\right] \cong H_{1}(X ; \mathbb{Z})$, onde $[$,$] denota$ o comutador de $\pi_{1}\left(X, x_{0}\right)$.

\section{B.2 Variedades $m$-homogêneas}

Esta seção foi motivada pela afirmação enunciada por Fadell e Neuwirth em (FADELL; NEUWIRTH, 1962), Section II, pg. 111, de que toda variedade topológica conexa $M$, com $\operatorname{dim} M \geq 2$, é $m$-homogênea, para todo $m$. Apresentaremos uma prova deste resultado, o qual é fundamental na demonstração de um dos principais teoremas deste trabalho (Teorema 3.1.25), garantindo que o espaço de configurações $F\left(M-Q_{m}, n\right)$ é essencialmente independente da escolha do conjunto $Q_{m}$. 
Definição B.2.1. ((UNGAR, 1978), Definition 2.2, pg. 155) Seja $m \geq 1$ um natural. Um espaço topológico $M$ é chamado fortemente $m$-homogêneo se dadas quaisquer duas $m$-uplas $\left(x_{1}, \ldots, x_{m}\right)$ e $\left(y_{1}, \ldots, y_{m}\right)$ de pontos distintos de $M$, existe um homeomorfismo $h: M \longrightarrow M$ tal que $h\left(x_{i}\right)=y_{i}$, para todo $i=1, \ldots, m$.

Observação B.2.2. Note que na Definição B.2.1, para as $m$-uplas $\left(x_{1}, \ldots, x_{m}\right)$ e $\left(y_{1}, \ldots, y_{m}\right)$ se tem $x_{i} \neq x_{j}$ e $y_{i} \neq y_{j}$, para todo $i \neq j$, com $i, j=1, \ldots, m$. Na linguagem adotada por Fadell e Neuwirth em (FADELL; NEUWIRTH, 1962), Section II, pg. 111, tais espaços são chamados, simplesmente, $m$-homogêneos.

Observação B.2.3. (i) Se $M$ é $m$-homogêneo, então $M$ é $k$-homogêneo, $\forall 1 \leq k \leq m$.

(ii) Se $M$ é $m$-homogêneo, então $M-\{p t o\}$ é $(m-1)$-homogêneo.

Exemplo B.2.4. Podemos mostrar que $\mathbb{R}$ é 1 e 2-homogêneo. Mas, provaremos que $\mathbb{R}$ não é $m$-homogêneo, $\forall m \geq 3$. Pela Observação B.2.3- $(i)$, basta provar que $\mathbb{R}$ não é 3 -homogêneo. Consideremos $x_{1}, x_{2}, x_{3}$ e $y_{1}=x_{1}, y_{2}=x_{2}, y_{3}$ números reais tal que $x_{1}<x_{2}<x_{3}$ e $y_{1}<y_{3}<y_{2}$ e suponha que exista um homeomorfismo $h: \mathbb{R} \longrightarrow \mathbb{R}$ que leva $x_{i}$ em $y_{i}, \quad i=1,2,3$. Então, como $h: \mathbb{R} \longrightarrow \mathbb{R}$ é uma aplicação contínua e injetora, segue que $h$ é ou crescente ou decrescente. Se $h$ for crescente, então $x_{2}<y_{3}$, o que é uma contradição, pois $y_{3}<x_{2}$. Se $h$ for decrescente, então $x_{2}<x_{1}$, o que é uma contradição, pois $x_{1}<x_{2}$. Logo, tal $h$ não existe. Portanto, $\mathbb{R}$ não é 3 -homogêneo.

A propriedade de um espaço ser $m$-homogêneo é um invariante topológico, como mostra o seguinte resultado.

Proposição B.2.5. Sejam $M, N$ espaços homeomorfos. Se $M$ é $m$-homogêneo então $N$ também é $m$-homogêneo.

Demonstração. Considere um homeomorfismo $\varphi: N \longrightarrow M$ e suponha $M m$-homogêneo. Provaremos que $N$ também é $m$-homogêneo. De fato, sejam $a_{1}, \ldots, a_{m}$ e $b_{1}, \ldots, b_{m}$ sequências de pontos distintos de $N$ e denotemos $x_{i}:=\varphi\left(a_{i}\right)$ e $y_{i}:=\varphi\left(b_{i}\right)$, para cada $i=1, \ldots, m$. Assim, $x_{1}, \ldots, x_{m}$ e $y_{1}, \ldots, y_{m}$ são pares de pontos distintos de $M, \operatorname{logo}$ existe um homeomorfismo $h: M \longrightarrow M$ tal que $h\left(x_{i}\right)=y_{i}, \forall i=1, \ldots, m$. Para concluir, definamos o homeomorfismo $g: N \longrightarrow N$ como a composta $g:=\varphi^{-1} \circ h \circ \varphi$ e segue que $g$ satisfaz,

$$
g\left(a_{i}\right)=\varphi^{-1} \circ h \circ \varphi\left(a_{i}\right)=\varphi^{-1} \circ h\left(x_{i}\right)=\varphi^{-1}\left(y_{i}\right)=b_{i}, \quad \forall i=1, \ldots, m
$$

Exemplo B.2.6. Podemos mostrar que $\mathbb{S}^{1}$ é 1,2 e 3 -homogênea. Mas, provaremos que $\mathbb{S}^{1}$ não é $m$-homogênea, $\forall m \geq 4$. Novamente pela Observação B.2.3-(i), basta provar 
que $\mathbb{S}^{1}$ não é 4-homogênea. Suponha que $\mathbb{S}^{1}$ seja 4-homogênea, então pela Observação B.2.3-(ii), $\mathbb{S}^{1}-\{p t o\}$ é 3-homogêneo, mas $\mathbb{S}^{1}-\{p t o\} \approx \mathbb{R}$ não é 3-homogêneo. Portanto, $\mathbb{S}^{1}$ não é 4-homogênea.

Observação B.2.7. Usando o fato ( vide (ROKHLIN; FUKS, 1977), Chapter 3, Section 1, pg. 140,141) que toda 1-variedade topológica conexa, se é compacta, então é homeomorfa a $\mathbb{S}^{1}$, e se não é compacta, então é homeomorfa a $\mathbb{R}$ (vide também Observação 1.4.19), e pelos Exemplos B.2.4,B.2.6 e a Proposição B.2.5, concluímos que toda 1-variedade conexa compacta não é $m$-homogênea, $\forall m \geq 3$ e, que toda 1 -variedade conexa não compacta não é $m$-homogênea, $\forall m \geq 4$.

Lema B.2.8. Toda $n$-variedade $M$ conexa, com $n \geq 2$, é 1 -homogênea.

Demonstração. Consideremos o grupo $(\operatorname{Top}(M), \circ)$, onde

$$
\operatorname{Top}(M):=\{h: M \longrightarrow M \mid h \text { é um homeomorfismo }\}
$$

é o conjunto dos homeomorfismos de $M$, munido da composição usual " o" de funções. A aplicação

$$
\begin{aligned}
\varphi: \operatorname{Top}(M) \times M & \longrightarrow M \\
(h, x) & \mapsto \varphi(h, x):=h(x)
\end{aligned}
$$

determina uma ação de $\operatorname{Top}(M)$ em $M$ (vide Definição 1.3.1) pois:

(i) $\varphi\left(I d_{M}, x\right)=i d_{M}(x)=x, \quad \forall x \in M$ com $I d_{M} \in T o p(M)$ o homeomorfismo identidade.

(ii) $\varphi(f, \varphi(g, x))=\varphi(f, g(x))=f(g(x))=(f \circ g)(x)=\varphi(f \circ g, x), \quad \forall f, g \in T o p(M) \mathrm{e}$ $\forall x \in M$.

Então,

$$
M=\bigcup_{x \in M} \operatorname{Top}(M) \cdot x
$$

onde $\operatorname{Top}(M) \cdot x:=\{h(x) \in M \mid h \in T o p(M)\}$ é a órbita de $x$.

Note que:

$\operatorname{Top}(M) \cdot x \subseteq M$ é aberto $\Longleftrightarrow \exists U \subseteq M$ aberto tal que $x \in U \subseteq T o p(M) \cdot x$ (B.2.1)

De fato, para a implicação $(\Longrightarrow)$ basta considerar $U:=\operatorname{Top}(M) \cdot x$. Reciprocamente, seja $z \in \operatorname{Top}(M) \cdot x$. Por hipótese, existe um aberto $U$ tal que $x \in U \subseteq \operatorname{Top}(M) \cdot x$. Seja $h \in \operatorname{Top}(M)$ tal que $h(z)=x \in U$. Então, $z \in h^{-1}(U) \subseteq M$ aberto. Mostremos que $h^{-1}(U) \subseteq T o p(M) \cdot x$. Se $y \in h^{-1}(U)$, então $h(y) \in U \subseteq T o p(M) \cdot x$. Considere $f \in \operatorname{Top}(M)$ tal que $h(y)=f(x)$. Logo

$y=\varphi\left(i d_{M}, y\right)=\varphi\left(h^{-1} \circ h, y\right)=\varphi\left(h^{-1}, h(y)\right)=\varphi\left(h^{-1}, f(x)\right)=\varphi\left(h^{-1} \circ f, x\right) \in \operatorname{Top}(M) \cdot x$. 
Assim, $y \in \operatorname{Top}(M) \cdot x$. Portanto, existe $h^{-1}(U)$ aberto tal que $z \in h^{-1}(U) \subseteq \operatorname{Top}(M) \cdot x$, ou seja, $\operatorname{Top}(M) \cdot x$ é aberto.

Agora, para cada $x \in M$ consideremos $\bar{U} \subseteq M$ vizinhança de disco para $x$ e a aplicação $\gamma: U \longrightarrow \operatorname{Top}_{s}(M)$ dada no Corolário 3.1.11. Para $z \in U, \gamma(z) \in T o p(M)$ e satisfaz $\gamma(z)(z)=x$, ou seja, $z=f(x)$, onde $f:=(\gamma(z))^{-1} \in \operatorname{Top}(M)$. Assim, $U \subseteq \operatorname{Top}(M) \cdot x$. Portanto, de (B.2.1), Top $(M) \cdot x$ é aberto, $\forall x \in M$, o que implica que $T o p(M) \cdot x$ é também fechado, $\forall x \in M$. Assim, como $M$ é conexa, temos

$$
M=T o p(M) \cdot x, \quad \forall x \in M
$$

Finalmente, para quaisquer $x, y \in M$, segue de (B.2.2), $y \in T o p(M) \cdot x$, ou seja, $y=h(x)$, para algum $h \in T o p(M)$. Portanto, $M$ é 1 -homogênea.

Teorema B.2.9. ((FADELL; NEUWIRTH, 1962), Section II, pg. 111) Toda $n$-variedade $M$ conexa, com $n \geq 2$, é $m$-homogênea para todo $m$.

Demonstração. A prova será feita por indução sobre $m$. Quando $m=1$ segue do Lema B.2.8. Suponhamos que $M$ seja $l$-homogênea, $\forall 1 \leq l \leq m-1$ e, provaremos que $M$ é $m$-homogênea. Sejam $x_{1}, \ldots, x_{m}$ e $y_{1}, \ldots, y_{m}$ sequências de pontos distintos de $M$ e consideremos o conjunto

$$
\operatorname{Top}_{x_{1}, \ldots, x_{m-1}}^{y_{1}, \ldots, y_{m-1}}(M):=\left\{h \in \operatorname{Top}(M) \mid \quad h\left(x_{i}\right)=y_{i}, \quad \forall 1 \leq i \leq m-1\right\}
$$

o qual por hipótese de indução é não vazio, isto é,

$$
\operatorname{Top}_{x_{1}, \ldots, x_{m-1}}^{y_{1}, \ldots, y_{m-1}}(M) \neq \emptyset
$$

Provaremos que o conjunto não vazio

$$
\operatorname{Top}_{x_{1}, \ldots, x_{m-1}}^{y_{1}, \ldots, y_{m-1}}(M) \cdot x_{m}:=\left\{h\left(x_{m}\right) \mid \quad h \in \operatorname{Top}_{x_{1}, \ldots, x_{m-1}}^{y_{1}, \ldots, y_{m-1}}(M)\right\} \subseteq M \backslash\left\{y_{1}, \ldots, y_{m-1}\right\}
$$

é aberto e fechado em $M \backslash\left\{y_{1}, \ldots, y_{m-1}\right\}$. Vejamos que

$$
\operatorname{Top}_{x_{1}, \cdots, x_{m-1}}^{y_{1}, \ldots, y_{m-1}}(M) \cdot x_{m}
$$

é aberto em $M$. Assim, em particular,

$$
\operatorname{Top}_{x_{1}, \ldots, x_{m-1}}^{y_{1}, \ldots, y_{m-1}}(M) \cdot x_{m}
$$

é aberto em $M \backslash\left\{y_{1}, \ldots, y_{m-1}\right\}$. De fato, seja

$$
z \in \operatorname{Top}_{x_{1}, \ldots, x_{m-1}}^{y_{1}, \ldots, y_{m-1}}(M) \cdot x_{m} .
$$

Então, existe

$$
h \in \operatorname{Top}_{x_{1}, \ldots, x_{m-1}}^{y_{1}, \ldots, y_{m-1}}(M)
$$


tal que

$$
z=h\left(x_{m}\right) \in M \backslash\left\{y_{1}, \ldots, y_{m-1}\right\} .
$$

Seja $\bar{U}$ uma vizinhança de disco para $z \in U$, com $U \subseteq M \backslash\left\{y_{1}, \ldots, y_{m-1}\right\}$. Mostraremos que

$$
U \subseteq T o p_{x_{1}, \ldots, x_{m-1}}^{y_{1}, \ldots, y_{m-1}}(M) \cdot x_{m}
$$

Dado $z^{\prime} \in U$, consideremos a aplicação

$$
\gamma: U \longrightarrow \operatorname{Top}_{s}(M)
$$

dada no Corolário 3.1.11. Temos que $\gamma\left(z^{\prime}\right)\left(z^{\prime}\right)=z$, em particular, $\left(\gamma\left(z^{\prime}\right)\right)^{-1} \in$ Top $(M)$ leva $z$ em $z^{\prime}$. Agora a composição

$$
\left(\gamma\left(z^{\prime}\right)\right)^{-1} \circ h \in \operatorname{Top}(M)
$$

satisfaz para cada $i=1, \ldots, m-1$,

$$
\begin{aligned}
\left(\gamma\left(z^{\prime}\right)\right)^{-1} \circ h\left(x_{i}\right) & =\left(\gamma\left(z^{\prime}\right)\right)^{-1}\left(y_{i}\right) \\
& =y_{i},
\end{aligned}
$$

onde a última igualdade segue de uma das propriedades da aplicação $\gamma$ (vide Corolário 3.1.11), pois $y_{i} \notin U, \quad \forall i=1 \ldots, m-1$. Assim,

$$
\left(\gamma\left(z^{\prime}\right)\right)^{-1} \circ h \in \operatorname{Top}_{x_{1}, \ldots, x_{m-1}}^{y_{1}, \ldots, y_{m-1}}(M)
$$

Além disso,

$$
\left(\gamma\left(z^{\prime}\right)\right)^{-1} \circ h\left(x_{m}\right)=\left(\gamma\left(z^{\prime}\right)\right)^{-1}(z)=z^{\prime}
$$

$\log O$

$$
z^{\prime}=\left(\gamma\left(z^{\prime}\right)\right)^{-1} \circ h\left(x_{m}\right) \in \operatorname{Top}_{x_{1}, \ldots, x_{m-1}}^{y_{1}, \ldots, y_{m-1}}(M) \cdot x_{m} .
$$

Isto mostra que $U \subseteq T o p_{x_{1}, \ldots, x_{m-1}}^{y_{1}, \ldots, y_{m-1}}(M) \cdot x_{m}$. Portanto,

$$
z \in U \subseteq T o p_{x_{1}, \ldots, x_{m-1}}^{y_{1}, \ldots, y_{m-1}}(M) \cdot x_{m}
$$

ou seja,

$$
\operatorname{Top}_{x_{1}, \cdots, x_{m-1}}^{y_{1}, \cdots, y_{m-1}}(M) \cdot x_{m}
$$

é aberto em $M$. Agora, vejamos que

$$
\operatorname{Top}_{x_{1}, \ldots, x_{m-1}}^{y_{1}, \ldots, y_{m-1}}(M) \cdot x_{m}
$$

é fechado em $M \backslash\left\{y_{1}, \cdots, y_{m-1}\right\}$. Seja

$$
z \in \overline{\operatorname{Top}_{x_{1}, \ldots, x_{m-1}}^{y_{1}, \ldots, y_{m-1}}(M) \cdot x_{m}} \subseteq M \backslash\left\{y_{1}, \ldots, y_{m-1}\right\}
$$


Seja $\bar{U}$ vizinhança de disco para $z \in U$, com $U \subseteq M \backslash\left\{y_{1}, \cdots, y_{m-1}\right\}$. Assim, $U$ é um aberto em $M \backslash\left\{y_{1}, \ldots, y_{m-1}\right\}$ e $z \in U, \log \mathrm{O}$

$$
\operatorname{Top}_{x_{1}, \ldots, x_{m-1}}^{y_{1}, \ldots, y_{m-1}}(M) \cdot x_{m} \cap U \neq \emptyset \text {. }
$$

Então, existe

$$
z^{\prime} \in \operatorname{Top}_{x_{1}, \ldots, x_{m-1}}^{y_{1}, \ldots, y_{m-1}}(M) \cdot x_{m} \cap U
$$

Seja

$$
h \in \operatorname{Top}_{x_{1}, \ldots, x_{m-1}}^{y_{1}, \ldots, y_{m-1}}(M)
$$

tal que $h\left(x_{m}\right)=z^{\prime} \in U$. Considerando a aplicação

$$
\gamma: U \longrightarrow \operatorname{Top}_{s}(M)
$$

dada no Corolário 3.1.11, obtemos $\gamma\left(z^{\prime}\right) \in \operatorname{Aut}(M)$ que leva $z^{\prime}$ em $z$. A composição,

$$
\gamma\left(z^{\prime}\right) \circ h \in T o p(M)
$$

satisfaz para cada $i=1, \ldots, m-1$,

$$
\gamma\left(z^{\prime}\right) \circ h\left(x_{i}\right)=\gamma\left(z^{\prime}\right)\left(y_{i}\right)=y_{i}
$$

novamente, a última igualdade segue das propriedades da aplicação $\gamma$ e, assim

$$
\gamma\left(z^{\prime}\right) \circ h \in \operatorname{Top}_{x_{1}, \ldots, x_{m-1}}^{y_{1}, \ldots, y_{m-1}}(M) .
$$

Além disso,

$$
\gamma\left(z^{\prime}\right) \circ h\left(x_{m}\right)=\gamma\left(z^{\prime}\right)\left(z^{\prime}\right)=z .
$$

Portanto,

$$
z \in \operatorname{Top}_{x_{1}, \ldots, x_{m-1}}^{y_{1}, \ldots, y_{m-1}}(M) \cdot x_{m},
$$

o que implica

$$
\overline{\operatorname{Top}_{x_{1}, \ldots, x_{m-1}}^{y_{1}, \ldots, y_{m-1}}(M) \cdot x_{m}}=\operatorname{Top}_{x_{1}, \ldots, x_{m-1}}^{y_{1}, \ldots, y_{m-1}}(M) \cdot x_{m},
$$

ou seja,

$$
\operatorname{Top}_{x_{1}, \ldots, x_{m-1}}^{y_{1}, \ldots, y_{m-1}}(M) \cdot x_{m}
$$

é fechado em

$$
M \backslash\left\{y_{1}, \cdots, y_{m-1}\right\} .
$$

Assim, mostramos que

$$
\operatorname{Top}_{x_{1}, \ldots, x_{m-1}}^{y_{1}, \ldots, y_{m-1}}(M) \cdot x_{m}
$$

é aberto e fechado não vazio em $M \backslash\left\{y_{1}, \ldots, y_{m-1}\right\}$, e como $M \backslash\left\{y_{1}, \ldots, y_{m-1}\right\}$ é conexa (pois $\operatorname{dim}(M) \geq 2$ ), segue que

$$
\operatorname{Top}_{x_{1}, \ldots, x_{m-1}}^{y_{1}, \ldots, y_{m-1}}(M) \cdot x_{m}=M \backslash\left\{y_{1}, \cdots, y_{m-1}\right\} .
$$

Assim, como

$$
y_{m} \in M \backslash\left\{y_{1}, \ldots, y_{m-1}\right\} \text {, existe } h \in \operatorname{Top}_{x_{1}, \ldots, x_{m-1}}^{y_{1}, \ldots, y_{m-1}}(M) \text { tal que } h\left(x_{m}\right)=y_{m} .
$$

Portanto, existe $h \in T o p(M)$ tal que $h\left(x_{i}\right)=y_{i}, \quad \forall i=1, \ldots, m$. 


\section{B.3 Sequência exata curta para um recobrimento}

O objetivo desta seção é provar que dado um $G$-espaço $E$ e um recobrimento $p: E \rightarrow B$, sob certas condições, existe uma sequência exata curta

$$
1 \longrightarrow \pi_{1}\left(E, e_{0}\right) \stackrel{p_{\star}}{\longrightarrow} \pi_{1}\left(B, b_{0}\right) \stackrel{q}{\longrightarrow} G \longrightarrow 1
$$

satisfazendo a condição de naturalidade (vide Teorema B.3.2). Além disso, mostraremos que dados $G$-espaços livres $E_{1}$ e $E_{2}$ tais que $p_{1}: E_{1} \rightarrow E_{1} / G$ e $p_{2}: E_{2} \rightarrow E_{2} / G$ são recobrimentos e uma aplicação $G$-equivariante $f: E_{1} \rightarrow E_{2}$, se $f$ for uma equivalência de homotopia fraca, então a aplicação $\bar{f}: E_{1} / G \rightarrow E_{2} / G$ entre os espaços de órbitas induzida por $f$ também será uma equivalência de homotopia fraca (vide Proposição B.3.4). Tais resultados são interessantes para se calcular o grupo fundamental de espaços de configurações não ordenados e também serão usados no Capítulo 5.

Observação B.3.1. Seja $E$ um $G$-espaço livre e denotemos o espaço de órbitas por $B=E / G$. Suponhamos que $p: E \longrightarrow B$ seja um recobrimento. Consideremos pontos base $e_{0} \in E$ e $b_{0}=p\left(e_{0}\right)=G e_{0} \in B$, onde $G e_{0}$ é a órbita do ponto $e_{0}$ pela ação de $G$, e a fibra:

$$
\begin{aligned}
p^{-1}\left(G e_{0}\right) & =\left\{x \in E \mid \quad p(x)=G e_{0}\right\} \\
& =\left\{x \in E \mid \quad G x=G e_{0}\right\} \\
& =\left\{x \in E \mid x \sim e_{0}\right\} \\
& =G e_{0},
\end{aligned}
$$

onde $G x$ denota a órbita de um ponto $x \in E$. Definimos a aplicação

$$
q: \pi_{1}\left(B, b_{0}\right) \longrightarrow G \text { por }[\lambda] \mapsto g_{\lambda}
$$

onde $g_{\lambda} \in G$ é construído como segue. Para cada $[\lambda] \in \pi_{1}\left(B, b_{0}\right)$, como $\lambda:[0,1] \longrightarrow B$ é um laço baseado em $b_{0}$, pelo Teorema do Levantamento de Caminhos ${ }^{1}$, existe um único levantamento $\tilde{\lambda}:[0,1] \longrightarrow E$ para $\lambda$ começando em $e_{0}$, ou seja, $p \circ \tilde{\lambda}=\lambda, \operatorname{com} \tilde{\lambda}(0)=e_{0}$. Note que, $\tilde{\lambda}(1) \in p^{-1}\left(G e_{0}\right)$ e como a ação de $G$ sobre $E$ é livre, existe um único $g:=g_{\lambda} \in G$ tal que $\tilde{\lambda}(1)=g_{\lambda} \cdot e_{0}$

Vejamos que $q$ é bem definida. Sejam $[\lambda],[\gamma] \in \pi_{1}\left(B, b_{0}\right)$ tais que $[\lambda]=[\gamma]$, ou seja, $\lambda, \gamma:[0,1] \longrightarrow B$ são laços em $b_{0}$ e $\lambda \simeq \gamma$ rel $\{0,1\}$. Novamente, pelo fato de $p$ ser um recobrimento, segue do Teorema da Monodromia ${ }^{2}$ que seus únicos levantamentos $\tilde{\lambda}, \tilde{\gamma}:[0,1] \longrightarrow E$ começando em $e_{0}$ possuem o mesmo ponto final e são caminhos homotópicos, ou seja,

$$
\tilde{\lambda}(0)=\tilde{\gamma}(0)=e_{0}, \quad \tilde{\lambda} \simeq \tilde{\gamma} \operatorname{rel}\{0,1\}, \quad \text { e } \quad \tilde{\lambda}(1)=\tilde{\gamma}(1)
$$

1 Vide (MUNKRES, 2002), Capítulo 9, Section 54, Lema 54.1, pg. 342.

2 Vide (MUNKRES, 2002), Capítulo 9, Section 54, Teorema 54.3, pg. 344. 
Assim, como a ação é livre, existem únicos $g_{\lambda}, g_{\gamma} \in G$ tais que $g_{\lambda} \cdot e_{0}=\tilde{\lambda}(1)=\tilde{\gamma}(1)=g_{\gamma} \cdot e_{0}$. Portanto, $g_{\lambda}=g_{\gamma}$.

Mostraremos que $q$ é um homomorfismo de grupos. Dados $[\gamma],[\lambda] \in \pi_{1}\left(B, b_{0}\right)$, então:

$$
\begin{aligned}
q([\gamma] *[\lambda]) & =q[\gamma * \lambda] \\
& =g_{\gamma * \lambda} .
\end{aligned}
$$

Consideremos o caminho $g_{\gamma} \cdot \tilde{\lambda}:[0,1] \longrightarrow E$, definido para todo $t \in[0,1]$ por:

$$
\left(g_{\gamma} \cdot \tilde{\lambda}\right)(t):=g_{\gamma} \cdot \tilde{\lambda}(t)
$$

Observemos que $g_{\gamma} \cdot \tilde{\lambda}$ é contínua, pois é dada pela composição:

$$
\begin{aligned}
{[0,1] } & \stackrel{\tilde{\lambda}}{\longrightarrow} E \stackrel{\varphi_{g_{\gamma}}}{\longrightarrow} E \\
t & \left.\mapsto \tilde{\lambda}(t) \mapsto \varphi_{g_{\gamma}}(\tilde{\lambda})(t)\right):=g_{\gamma} \cdot \tilde{\lambda}(t)
\end{aligned}
$$

onde $\varphi: G \times E \longrightarrow E$ é a ação livre de $G$ sobre $E$, logo $\varphi_{g_{\gamma}}: E \longrightarrow E$ é contínua. Por outro lado:

$$
\begin{aligned}
\left(g_{\gamma} \cdot \tilde{\lambda}\right)(0) & =g_{\gamma} \cdot \tilde{\lambda}(0) \\
& =g_{\gamma} \cdot e_{0}=\tilde{\gamma}(1) . \\
\left(g_{\gamma} \cdot \tilde{\lambda}\right)(1) & =g_{\gamma} \cdot \tilde{\lambda}(1) \\
& =\left(g_{\gamma} g_{\lambda}\right) \cdot e_{0} .
\end{aligned}
$$

Assim, como podemos considerar a concatenação dos caminhos $\tilde{\gamma}$ e $\left(g_{\gamma} \cdot \tilde{\lambda}\right)$ e, dessa forma, obtemos o caminho:

$$
\tilde{\gamma} *\left(g_{\gamma} \cdot \tilde{\lambda}\right):[0,1] \longrightarrow E
$$

o qual satisfaz:

$$
\tilde{\gamma} *\left(g_{\gamma} \cdot \tilde{\lambda}\right)(0)=\tilde{\gamma}(0)=e_{0}
$$

Além disso,

$$
\begin{aligned}
\left(p \circ\left(\tilde{\gamma} *\left(g_{\gamma} \cdot \tilde{\lambda}\right)\right)(t)\right. & = \begin{cases}p \circ \tilde{\gamma}(2 t), & 0 \leq t \leq \frac{1}{2} \\
\underbrace{p \circ\left(g_{\gamma} \cdot \tilde{\lambda}\right)(2 t-1)}_{p(g \cdot x)=p(x)}, & \frac{1}{2} \leq t \leq 1 .\end{cases} \\
& = \begin{cases}p \circ \tilde{\gamma}(2 t), & 0 \leq t \leq \frac{1}{2} \\
p \circ \tilde{\lambda}(2 t-1), & \frac{1}{2} \leq t \leq 1 .\end{cases} \\
& = \begin{cases}\gamma(2 t), & 0 \leq t \leq \frac{1}{2} \\
\lambda(2 t-1), & \frac{1}{2} \leq t \leq 1 .\end{cases} \\
& =(\gamma * \lambda)(t) .
\end{aligned}
$$


Assim, $\tilde{\gamma} *\left(g_{\gamma} \cdot \tilde{\lambda}\right)$ é um levantamento para o caminho $\gamma * \lambda$ começando em $e_{0}$. Pela unicidade do levantamento,

$$
\widetilde{\gamma * \lambda}=\tilde{\gamma} *\left(g_{\gamma} \cdot \tilde{\lambda}\right)
$$

Então,

$$
\begin{aligned}
\widetilde{\gamma * \lambda}(1) & =\left(\tilde{\gamma} *\left(g_{\gamma} \cdot \tilde{\lambda}\right)\right)(1) \\
& =\left(g_{\gamma} \cdot \tilde{\lambda}\right)(1) \\
& =g_{\gamma} \cdot \tilde{\lambda}(1) \\
& =g_{\gamma}\left(g_{\lambda} \cdot e_{0}\right) \\
& =\left(g_{\gamma} g_{\lambda}\right) \cdot e_{0} .
\end{aligned}
$$

Logo, $g_{\gamma * \lambda}=g_{\gamma} g_{\lambda}$. De (B.3.1) obtemos:

$$
\begin{aligned}
q([\gamma] *[\lambda]) & =g_{\gamma} g_{\lambda} \\
& =q[\gamma] q[\lambda]
\end{aligned}
$$

Com as notações da Observação B.3.1. obtemos o seguinte resultado.

Teorema B.3.2. Seja $E$ um $G$-espaço livre conexo por caminhos tal que a projeção $p: E \longrightarrow B:=E / G$ é um recobrimento. Então, existe uma sequência exata curta

$$
1 \longrightarrow \pi_{1}\left(E, e_{0}\right) \stackrel{p_{\star}}{\longrightarrow} \pi_{1}\left(B, b_{0}\right) \stackrel{q}{\longrightarrow} G \longrightarrow 1,
$$

a qual é natural com respeito a quadrados comutativos, como na Figura 29,

Figura 29 - Naturalidade do recobrimento

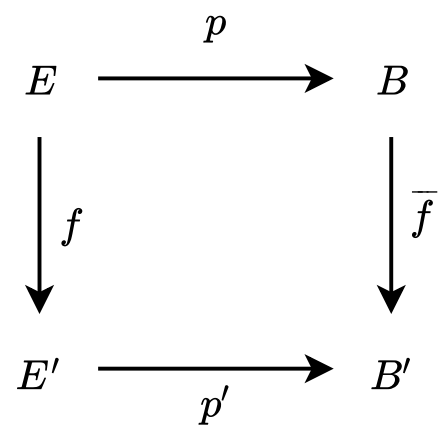

Fonte: Elaborada pelo autor.

onde $p$ e $p^{\prime}$ são recobrimentos, com $E^{\prime}$ um $G$-espaço livre conexo por caminhos, $f: E \rightarrow E^{\prime}$ é uma aplicação $G$-equivariante tal que $f\left(e_{0}\right)=e_{1}$ e

$$
\bar{f}: B=E / G \longrightarrow B^{\prime}=E^{\prime} / G
$$

é a aplicação induzida por $f$ nos espaços de órbitas. A naturalidade significa que o diagrama da Figura 30 é comutativo. 
Figura 30 - Naturalidade do diagrama da sequência associada a um recobrimento

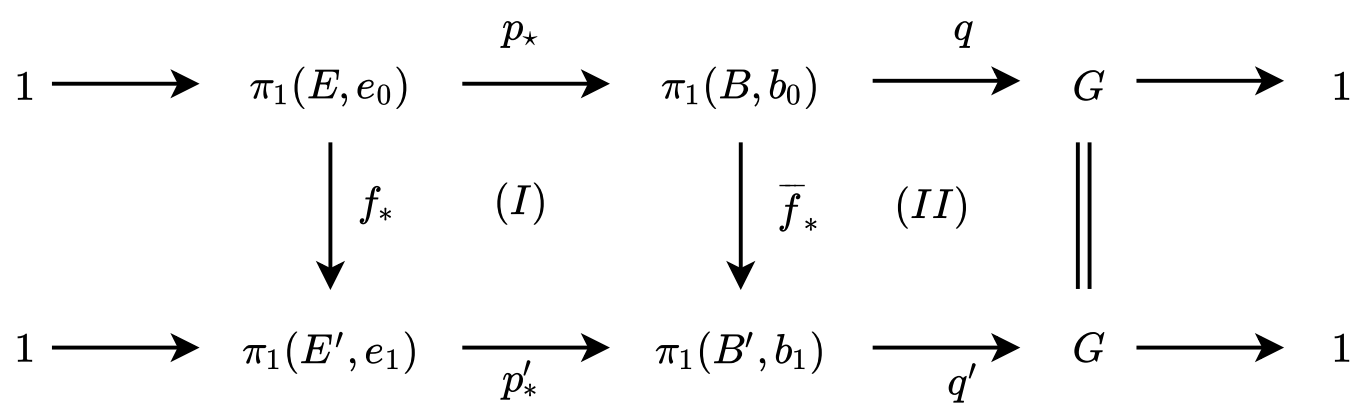

Fonte: Elaborada pelo autor.

Demonstração. Vejamos que $p_{*}$ é injetora. Seja $[\lambda] \in \pi_{1}\left(E, e_{0}\right)$ tal que $p_{\star}[\lambda]=\left[C_{b_{0}}\right]$, ou seja, $[p \circ \lambda]=\left[C_{b_{0}}\right]$, onde $C_{b_{0}}$ denota o laço constante em $b_{0}$. Assim

$$
p \circ \lambda \simeq C_{b_{0}} \operatorname{rel}\{0,1\} \text { em B. }
$$

Do Teorema do Levantamento de Caminhos e Monodromia, existem únicos levantamentos:

$$
\widetilde{p \circ \lambda} \text { e } \widetilde{C_{b_{0}}}
$$

para $p \circ \lambda$ e $C_{b_{0}}$, respectivamente, começando em $e_{0}$. Além disso,

$$
\widetilde{p \circ \lambda} \simeq \widetilde{C_{b_{0}}} \operatorname{rel}\{0,1\} \text { em E. }
$$

Mas, $\widetilde{p \circ \lambda}=\lambda$ e $\widetilde{C_{b_{0}}}=C_{e_{0}}$. Portanto, $[\lambda]=\left[C_{e_{0}}\right]$.

Mostremos que $q$ é sobrejetor. Dado $g \in G$, como $E$ é conexo por caminhos, existe

$$
\gamma:[0,1] \longrightarrow E
$$

um caminho em $E$ unindo $e_{0}$ a $g \cdot e_{0}$. Então, a composição

$$
\lambda:=p \circ \gamma:[0,1] \longrightarrow B
$$

é um caminho em $B$, satisfazendo:

$$
\begin{aligned}
& \lambda(0)=(p \circ \gamma)(0)=p\left(e_{0}\right)=b_{0} \text { e } \\
& \lambda(1)=(p \circ \gamma)(1)=p\left(g e_{0}\right)=G \cdot\left(g e_{0}\right)=G \cdot e_{0}=b_{0} .
\end{aligned}
$$

Logo, $\lambda$ é um laço em $B$ com ponto base $b_{0}$, ou seja, $[\lambda] \in \pi_{1}\left(B, b_{0}\right)$. Note que:

$$
\widetilde{\lambda}=\widetilde{p \circ \gamma}=\gamma \text {. }
$$

Então, $\tilde{\lambda}(1)=\gamma(1)=g \cdot e_{0}$ e, portanto, $q[\lambda]=g$. 
Resta provar que $\operatorname{Ker}(q)=\operatorname{Im}\left(p_{\star}\right)$. Seja $[\lambda] \in \pi_{1}\left(E, e_{0}\right)$. Então:

$$
\left(q \circ p_{*}\right)[\lambda]=q[p \circ \lambda]=g_{p \circ \lambda}=1
$$

A última igualdade é válida pois como $\widetilde{p \circ \lambda}=\lambda$, então:

$$
\widehat{(p \circ \lambda)}(1)=\lambda(1)=e_{0}=1 \cdot e_{0}, \quad \text { assim, } \quad g_{p \circ \lambda}=1 .
$$

Portanto, $\operatorname{Im}\left(p_{*}\right) \subseteq \operatorname{Ker}(q)$.

Por outro lado, dado $[\lambda] \in \operatorname{Ker}(q) \subseteq \pi_{1}\left(B, b_{0}\right)$, então, $q[\lambda]=g_{\lambda}=1$. Assim, o único levantamento $\tilde{\lambda}$ de $\lambda$ começando em $e_{0}$ satisfaz

$$
\widetilde{\lambda}(1)=g_{\lambda} \cdot e_{0}=1 \cdot e_{0}=e_{0},
$$

ou seja, $\tilde{\lambda}$ é um laço em $E$ com ponto base $e_{0}$, o que implica que

$$
[\tilde{\lambda}] \in \pi_{1}\left(E, e_{0}\right) \text { e } p_{\star}[\widetilde{\lambda}]=[p \circ \tilde{\lambda}]=[\lambda] .
$$

Portanto, $[\lambda] \in \operatorname{Im}\left(p_{*}\right)$ e $\operatorname{Ker}(q) \subseteq \operatorname{Im}\left(p_{*}\right)$.

Finalmente, mostremos que vale a comutatividade do diagrama da Figura 30. A comutatividade do quadrado $(I)$ à esquerda segue da comutatividade do quadrado da Figura 29. Resta provar a comutatividade do quadrado $(I I)$ à direita.

Se $\lambda:[0,1] \longrightarrow B$ é um laço em $B$ com ponto base $b_{0}$, ou seja, $[\lambda] \in \pi_{1}\left(B, b_{0}\right)$, então:

$$
\bar{f}_{*}[\lambda]=[\bar{f} \circ \lambda]
$$

Note que se $\widetilde{\lambda}$ é o único levantamento de $\lambda$ começando em $e_{0}$, então

$$
f \circ \tilde{\lambda}:[0,1] \longrightarrow E^{\prime}
$$

é um caminho em $E^{\prime}$ com ponto base $f\left(e_{0}\right)=e_{1}$, ou seja,

$$
(f \circ \widetilde{\lambda})(0)=f(\widetilde{\lambda(0)})=f\left(e_{0}\right)=e_{1} .
$$

Além disso,

$$
\underbrace{p^{\prime} \circ f}_{\bar{f} \circ p} \circ \tilde{\lambda}=\bar{f} \circ p \circ \tilde{\lambda}=\bar{f} \circ \lambda .
$$

Assim, $f \circ \tilde{\lambda}$ é um levantamento para $\bar{f} \circ \lambda$, começando em $e_{1}$. Segue da unicidade do levantamento de caminhos que

$$
\widetilde{\bar{f} \circ \lambda}:=f \circ \tilde{\lambda} \text {. }
$$

Então, segue do fato de $f$ ser uma aplicação $G$-equivariante, que:

$$
g_{\bar{f} \circ \lambda} \cdot e_{1}=(\widetilde{\bar{f} \circ \lambda})(1)=f \circ \tilde{\lambda}(1)=f\left(g_{\lambda} \cdot e_{0}\right)=g_{\lambda} \cdot f\left(e_{0}\right)=g_{\lambda} \cdot e_{1},
$$

Portanto,

$$
\left(q^{\prime} \circ \bar{f}_{*}\right)[\lambda]=q^{\prime}[\bar{f} \circ \lambda]=g_{\bar{f} \circ \lambda}=g_{\lambda}=q[\lambda] .
$$


Corolário B.3.3. Seja $E$ um $G$-espaço livre conexo por caminhos tal que a projeção ao quociente $p: E \longrightarrow B:=E / G$ é um recobrimento. Se $E$ for simplesmente conexo, então

$$
\pi_{1}(B) \cong G
$$

Proposição B.3.4. Sejam $E_{1}, E_{2} \quad G$-espaços com pontos base $e_{1} \in E_{1}, e_{2} \in E_{2}$ e $f: E_{1} \longrightarrow E_{2}$ uma aplicacão $G$-equivariante que preserva pontos base.

(1) O diagrama da Figura 31 é comutativo,

Figura 31 - Diagrama comutativo de aplicações quocientes.

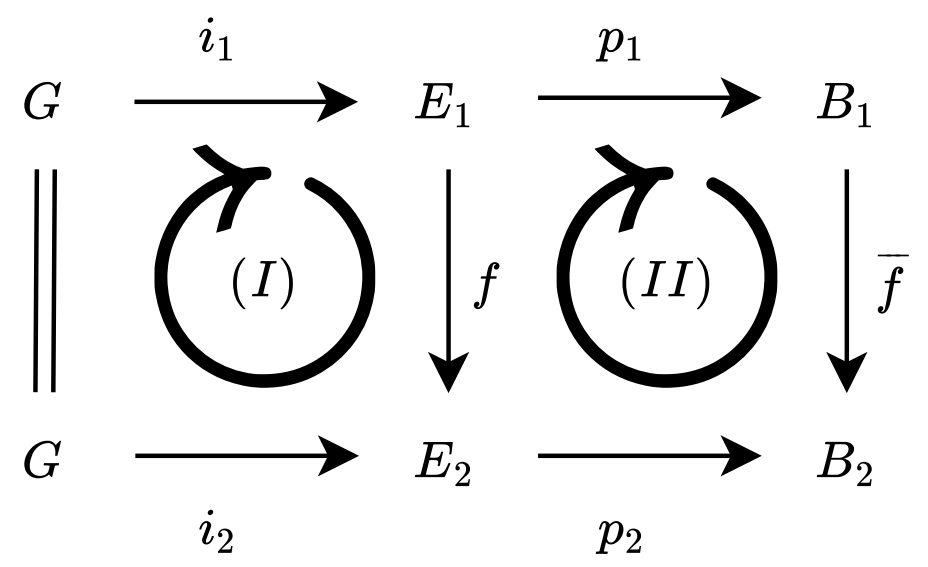

Fonte: Elaborada pelo autor.

onde

$$
i_{k}: G \longrightarrow E_{k}, \quad i_{k}(g):=g \cdot e_{k}, \quad k=1,2
$$

é a inclusão natural, $B_{k}:=E_{k} / G$ é o espaço quociente, $p_{k}: E_{k} \longrightarrow B_{k}$ é a projeção ao quociente e $\bar{f}: B_{1} \longrightarrow B_{2}$ é a induzida por $f$ nos espaços quocientes.

(2) Suponhamos que a ação de $G$ sobre os espaços $E_{1}$ e $E_{2}$ seja livre, com $E_{1}$ e $E_{2}$ conexos por caminhos tais que $p_{1}$ e $p_{2}$ são recobrimentos. Se $f$ é uma equivalência de homotopia fraca, então $\bar{f}$ também será uma equivalência de homotopia fraca.

Demonstração. Mostremos que são válidos (1) e (2).

(1) Usando a definição de $i_{k}$ e o fato de $f$ ser $G$-equivariante e preservar pontos base temos:

$$
\left(f \circ i_{1}\right)(g)=f\left(g \cdot e_{1}\right)=g \cdot f\left(e_{1}\right)=g \cdot e_{2}=i_{2}(g), \quad \forall g \in G \text {. }
$$

Assim, $f \circ i_{1}=i_{2}$ e o quadrado $(I)$ é comutativo.

Para mostrar que vale a comutatividade do quadrado $(I I)$, dado $x \in E$,

$$
\left(\bar{f} \circ p_{1}\right)(x)=\bar{f}(G \cdot x)=G \cdot f(x)=p_{2}(f(x)) .
$$


Assim, $\bar{f} \circ p_{1}=p_{2} \circ f$.

(2) Usando a naturalidade da sequência exata longa dos grupos de homotopia da fibração de Serre (Teorema 3.1.22), obtemos o diagrama comutativo da Figura 32.

Figura 32 - Usando a naturalidade da sequência exata longa de uma fibração.

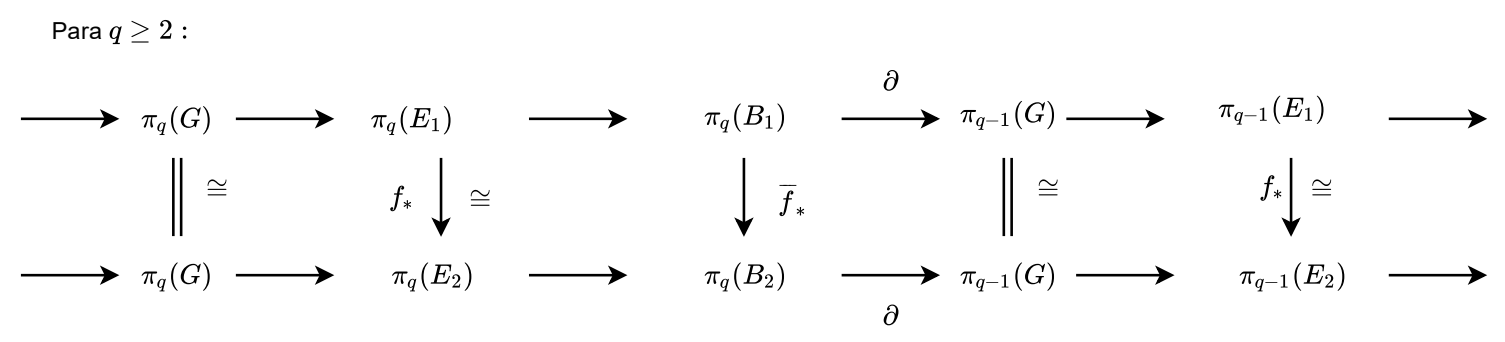

Fonte: Elaborada pelo autor.

Aplicando o Lema dos Cinco (Corolário A.0.2), concluímos que $\bar{f}_{*}$ é isomorfismo $\forall q \geq 2$. Agora, usando a naturalidade da sequência exata curta em grupos fundamentais para um recobrimento (vide Teorema B.3.2), obtemos o diagrama comutativo da Figura 33.

Figura 33 - Usando a naturalidade da sequência exata curta para um recobrimento.

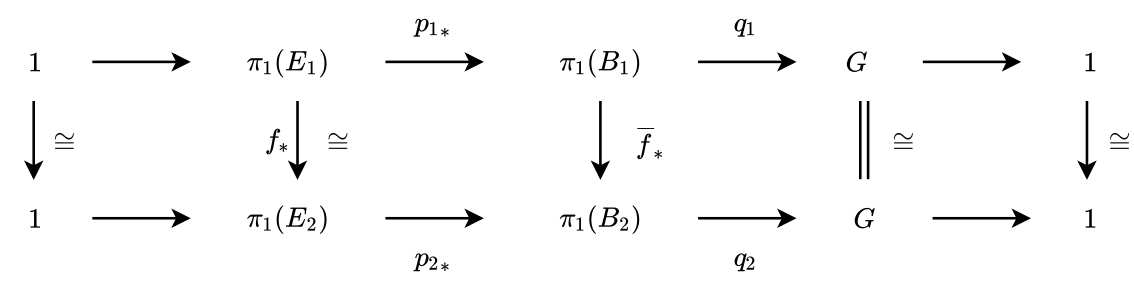

Fonte: Elaborada pelo autor.

Pelo Corolário A.0.2, $\bar{f}$ induz isomorfismo entre grupos fundamentais. Além disso, como $B_{1}$ e $B_{2}$ são conexos por caminhos, então $\bar{f}$ também induz "isomorfismo" em $\pi_{0}$.

Dessa forma, $\bar{f}$ induz isomorfismos entre grupos de homotopia e, portanto, $\bar{f}$ é uma equivalência de homotopia fraca.

\section{B.4 Variedades com buracos}

Nosso objetivo nesta seção é mostrar que o complementar de $r$ pontos no espaço Euclideano $\mathbb{R}^{n+1}$ tem o mesmo tipo de homotopia de um buquê de $r$-esferas $\mathbb{S}^{n}$, para todo $n+1 \geq 2$ e para todo $r \geq 1$.

Proposição B.4.1. (vide (HUSSEINI, 2002), Proposition 3.1, pg. 27) Sejam $M$ uma variedade topológicade dimensão $n+1 \geq 2$ e $Q_{r+1} \subseteq M$ um subconjunto discreto com 
$r+1$ elementos tal que $Q_{r+1} \subseteq V \subseteq D \subseteq M$, onde $D$ é um $(n+1)$-disco fechado em $M$ e $V$ denota seu interior. Então existe uma equivalência de homotopia

$$
(M-V) \vee\left(\mathbb{S}_{1} \vee \cdots \vee \mathbb{S}_{r}\right) \longrightarrow\left(M-Q_{r+1}\right)
$$

onde $\mathbb{S}_{1}, \cdots, \mathbb{S}_{r}$ denotam esferas $n$-dimensionais.

Usando a Proposição B.4.1, podemos calcular o tipo de homotopia do complementar de $r$ pontos no espaço Euclideano $\mathbb{R}^{n+1}$.

Corolário B.4.2. Para todo $n+1 \geq 2$ e para todo $r \geq 1$,

$$
\mathbb{R}^{n+1}-\{r \text { pontos }\} \simeq \bigvee^{r} \mathbb{S}^{n}
$$

Demonstração. Pela Proposição B.4.1, é suficiente mostrar que

$$
\mathbb{R}^{n+1}-V \simeq \mathbb{S}^{n}
$$

Definimos a aplicação $r: \mathbb{R}^{n+1}-V \longrightarrow \mathbb{S}^{n}$ pela expressão:

$$
r(x):=\frac{x}{\|x\|}, \quad \forall x \in \mathbb{R}^{n+1}-V .
$$

Assim, $r \circ i=I d_{\mathbb{S}^{n}}$, onde $\quad i: \mathbb{S}^{n} \hookrightarrow \mathbb{R}^{n+1}-V$ é a inclusão natural. Além disso,

$$
i \circ r \stackrel{H}{\simeq} I d_{\mathbb{R}^{n+1}-V}
$$

com a homotopia linear $H:\left(\mathbb{R}^{n+1}-V\right) \times I \longrightarrow \mathbb{R}^{n+1}-V$ definida como segue:

$$
H(x, t):=(1-t) \frac{x}{\|x\|}+t x, \quad \forall x \in \mathbb{R}^{n+1}-V .
$$



AGUILAR, M.; GITLER, S.; PRIETO, C. Algebraic Topology from a Homotopical Viewpoint. [S.l.]: Springer Science \& Business Media, 2002. Citado 7 vezes nas páginas $32,33,34,35,37,83$ e 84 .

ARNOL'D, V. I. The cohomology ring of the colored braid group. Mathematical Notes, Springer, v. 5, n. 2, p. 138-140, 1969. Citado na página 60.

BENDERSKY, M.; GITLER, S. The cohomology of certain function spaces. Transactions of the American Mathematical Society, v. 326, n. 1, p. 423-440, 1991. Citado 2 vezes nas páginas 22 e 58 .

BIRMAN, J. S. On braid groups. Communications on Pure and Applied Mathematics, Wiley Online Library, v. 22, n. 1, p. 41-72, 1969. Citado na página 93. página 94.

Braids, links and mapping class groups. Princeton Univ. Press, 1974. Citado na

BÖDIGHEIMER, C.-F.; COHEN, F.; TAYLOR, L. On the homology of configuration spaces. Topology, Elsevier, v. 28, n. 1, p. 111-123, 1989. Citado 4 vezes nas páginas 21, 57,68 e 74 .

BREDON, G.-E. Introduction to compact transformation groups. Academic Press, Bolyai Janos Matematikai Tarsulat, 1976. Citado na página 41.

BROWN, M. Locally flat imbeddings of topological manifolds. Annals of Mathematics, JSTOR, p. 331-341, 1962. Citado na página 71.

COHEN, F. Cohomology of braid spaces. Bulletin of the American Mathematical Society, v. 79, n. 4, p. 763-766, 1973. Citado 2 vezes nas páginas 60 e 114.

. The homology of $c_{n+1}$-spaces, $n \geq 0$. In: The homology of iterated loop spaces. [S.l.]: Springer, 1978. p. 207-351. Citado na página 114.

COHEN, F.; PAKIANATHAN, J. Configuration Spaces and Braid Groups. [S.l.]: Citeseer. Citado 8 vezes nas páginas 59, 60, 62, 86, 88, 91, 93 e 94.

COHEN, F. R. On configuration spaces, their homology, and lie algebras. Journal of Pure and Applied Algebra, Elsevier, v. 100, n. 1, p. 19-42, 1995. Citado 3 vezes nas páginas 58, 59 e 123 .

Introduction to configuration spaces and their applications. In: WORLD SCIENTIFIC. Braids. [S.1.], 2010. p. 183-261. Citado 6 vezes nas páginas 58, 60, 93, 104, 122 e 129.

DAVIS, J. F.; KIRK, P. Lecture notes in algebraic topology. [S.l.]: American Mathematical Society Providence, 2001. v. 35. Citado 4 vezes nas páginas 52, 66, 83 e 84. 
DEHORNOY, P.; DYNNIKOV, I.; ROLFSEN, D.; WIEST, B. Ordering braids. [S.l.]: American Mathematical Soc., 2008. Citado na página 108.

DIECK, T. tom. Transformation groups. [S.1.]: Walter de Gruyter, 1987. v. 8. Citado na página 107.

Algebraic topology. [S.1.]: European Mathematical Society, 2008. Citado 2 vezes nas páginas 41 e 133.

FADELL, E.; NEUWIRTH, L. Configuration spaces. Math. Scand, v. 10, n. 111-118, p. 4, 1962. Citado 17 vezes nas páginas 21, 25, 57, 60, 65, 90, 91, 94, 108, 109, 110, 111, 122, 133, 136, 137 e 139.

FADELL, E. R.; HUSSEINI, S. Y. Geometry and topology of configuration spaces. [S.l.]: Springer Science \& Business Media, 2001. Citado 5 vezes nas páginas 77, 78, 79, 81 e 86 .

FOX, R.; NEUWIRTH, L. The braid groups. Mathematica Scandinavica, JSTOR, v. 10, p. 119-126, 1962. Citado 2 vezes nas páginas 21 e 57.

FUCHS, D.; VIRO, O. Y. Topology II: Homotopy and Homology. Classical Manifolds. [S.l.]: Springer Science \& Business Media, 2003. v. 24. Citado 2 vezes nas páginas 90 e 91.

FUKS, D. Cohomologies of the group cos mod 2. Functional Analysis and its Applications, Springer, v. 4, n. 2, p. 143-151, 1970. Citado 2 vezes nas páginas 60 e 122.

HATCHER, A. Algebraic topology. [S.l.: s.n.], 2002. Citado 19 vezes nas páginas 41, 42, 44, 45, 53, 54, 55, 71, 83, 84, 90, 91, 93, 107, 113, 116, 128, 135 e 136.

HOVEY, M. Model categories. [S.l.]: American Mathematical Soc., 1999. Citado na página 85.

HUSSEINI, E. F. Configuration spaces on punctured manifolds. Journal of the Juliusz Schauder Center, v. 20, p. 25-42, 2002. Citado na página 148.

KAWAKUBO, K. The theory of transformation groups. [S.1.]: Oxford University Press, 1991. Citado 6 vezes nas páginas 38, 39, 40, 41, 43 e 103.

KIRBY, R. C. The topology of 4-manifolds. Springer, 1989. Citado na página 118.

KONTSEVICH, M. Operads and motives in deformation quantization. Letters in Mathematical Physics, Springer, v. 48, n. 1, p. 35-72, 1999. Citado na página 121.

KURLIN, V. et al. Computing braid groups of graphs with applications to robot motion planning. Homology, Homotopy and Applications, International Press of Boston, v. 14, n. 1, p. 159-180, 2012. Citado na página 68.

LEE, J. Introduction to topological manifolds. [S.1.]: Springer Science \& Business Media, 2010. v. 940. Citado 6 vezes nas páginas 33, 34, 44, 46, 47 e 134.

Introduction to Smooth Manifolds. [S.1.]: Springer Science \& Business Media, 2012. v. 218. Citado 7 vezes nas páginas 45, 46, 47, 65, 66, 68 e 70. 
LONGONI, R.; SALVATORE, P. Configuration spaces are not homotopy invariant. Topology, Elsevier, v. 44, n. 2, p. 375-380, 2005. Citado na página 63.

MACLANE, S. Categories for the working mathematician. Springer, 1971. Citado 4 vezes nas páginas 27, 28, 30 e 31 .

MASSEY, W. S. A basic course in algebraic topology. [S.l.]: Springer Science \& Business Media, 1991. Citado na página 136.

MCCLEARY, J. A user's guide to spectral sequences. [S.l.]: Cambridge University Press, 2001. Citado 2 vezes nas páginas 51 e 90.

MILGRAM, R. J.; LÖFFLER, P. The structure of deleted symmetric products. Braids (Santa Cruz, CA, 1986), v. 78, p. 415-424, 1988. Citado 2 vezes nas páginas 21 e 57.

MILNOR, J. Construction of universal bundles, ii. Annals of Mathematics, JSTOR, p. 430-436, 1956. Citado 2 vezes nas páginas 54 e 55.

MOISE, E. E. Geometric topology in dimensions 2 and 3. [S.l.]: Springer Science \& Business Media, 2013. v. 47. Citado na página 48.

MUNKRES, J. Topologia 2E. [S.l.: s.n.], 2002. Citado na página 142.

MUNKRES, J. R. Elements of algebraic topology. [S.l.]: Addison-Wesley Reading, 1984. v. 2. Citado na página 48.

ROBINSON, D. J. An introduction to abstract algebra. [S.l.]: Walter de Gruyter, 2003. Citado na página 97.

ROKHLIN, V.; FUKS, D. Beginner's Course in Topology. [S.l.]: Nauka, Moscow, 1977. Citado na página 138.

ROTH, F. On the category of euclidean configuration spaces and associated fibrations. Groups, homotopy and configuration spaces, v. 13, p. 447-461, 2008. Citado na página 121.

SALVATORE, P. Homotopy type of euclidean configuration spaces. Proceedings of the 20th Winter School"Geometry and Physics", Circolo Matematico di Palermo, p. 161-164, 2001. Citado na página 122.

SEGAL, G. Configuration-spaces and iterated loop-spaces. Inventiones Mathematicae, Springer, v. 21, n. 3, p. 213-221, 1973. Citado na página 121.

SELICK, P. Introduction to homotopy theory. [S.1.]: American Mathematical Soc., 2008. v. 9. Citado 5 vezes nas páginas 27, 28, 30, 101 e 132.

SHASTRI, A. R. Elements of differential topology. [S.l.]: CRC Press, 2011. Citado 3 vezes nas páginas 45, 47 e 48.

SPANIER, E.-H. Algebraic topology. McGraw-Hill Book Co., Interscience John Wiley \& Sons, 1966. Citado na página 85.

STEENROD, N. E. The topology of fibre bundles. [S.l.]: Princeton University Press, 1951. v. 14. Citado 2 vezes nas páginas 41 e 53. 
TOTARO, B. Configuration spaces of algebraic varieties. Topology, Elsevier, v. 35, n. 4, p. 1057-1067, 1996. Citado 2 vezes nas páginas 22 e 58.

UNGAR, G. S. Countable dense homogeneity and n-homogeneity. Fundamenta Mathematicae, Institute of Mathematics Polish Academy of Sciences, v. 99, n. 3, p. 155-160, 1978. Citado na página 137.

VICK, J. W. Homology Theory: An Introduction to Algebraic Topology. [S.l.]: Springer Science \& Business Media, 1994. v. 145. Citado na página 135.

WEIBEL, C. A. An introduction to homological algebra. Cambridge university press, 1994. Citado 7 vezes nas páginas 28, 30, 31, 32, 49, 50 e 51 .

WESTERLAND, C. Configuration spaces in topology and geometry. The Australian Mathematical Society, p. 279, 2012. Citado na página 121.

WHITEHEAD, G. W. Elements of homotopy theory. Springer-Verlag, 1978. Citado 2 vezes nas páginas 114 e 115.

WHITEHEAD, J. H. C. A certain open manifold whose group is unity. The Quarterly Journal of Mathematics, Oxford Univ Press, n. 1, p. 268-279, 1935. Citado na página 118.

ZARISKI, O. The topological discriminant group of a riemann surface of genus p. American Journal of Mathematics, JSTOR, v. 59, n. 2, p. 335-358, 1937. Citado 2 vezes nas páginas 21 e 57. 
$G$-ação, 38

$G$-conjunto, 38

$G$-espaço, 38

$G$-isomorfismo, 43

$R$-módulo filtrado, 49

$k$-esqueleto, 48

n-conexo, 53

órbita, 39

ação $m$-transitiva, 40

ação efetiva, 40

ação livre, 40

ação propriamente descontínua, 41

ação semi-livre, 40

ação transitiva, 40

ação trivial, 40

aplicação $G$-equivariante, 43

aplicação aberta, 41

aplicação associação, 36

aplicação avaliação, 33

aplicação concatenação, 37

aplicações não coincidentes, 110

bordo de uma variedade, 69

célula fechada, 48

característica de Euler, 126

Categoria, 27

categoria concreta, 31

conjunto $G$-invariante, 43

conjunto dos pontos fixos de $X$ pela ação de $G, 39$

conjunto relativamente compacto, 33

CW complexo, 48 disco fechado em uma variedade, 80

equivalência de categorias, 32

equivalência de homotopia fraca, 113

equivalência natural, 30

espaço classificante, 54

espaço de órbitas, 41

espaço de configurações clássicos, 58

espaço de configurações não ordenado, 59

Espaço de configurações ordenado, 58

espaço de Eilenberg-MacLane, 53, 90

espaço localmente compacto, 34

espaço localmente Euclideano, 44

espaço topológico homogêneo, 137

fibração, 84

Fibração de Hurewicz, 84

fibração de Hurewicz, 84

Fibração de Serre, 84

fibração de Serre, 84

fibrado, 53

fibrado $n$-universal, 53

fibrado localmente trivial, 83

fibrado principa, 53

fibrado principal, 53

fibrado universal, 53

filtração crescente, 49

filtração decrescente, 49

functor fiel, 31

functor inclusão, 31

functores de configurações, 29

funtor, 28

funtor completamente fiel, 32

funtor completo, 32 
funtor contravariante, 29

Funtor esquecimento, 31

funtor identidade, 31

grupo de isotropia, 39

homeomorfismo estável, 77

homomorfismo de Hurewicz, 135

interior de uma variedade, 69

isomorfismo, 28

isomorfismo natural, 30

isotopia, 62

lema da cisão, 101

levantamento de caminhos, 142

localmente conexo por caminhos, 44

monoide, 28

morfismo de sequências esprectrais, 50

números de Betti, 125

Polinômio de Poincaré, 126

ponto de bordo, 69

ponto fixo, 110

ponto interior, 69

produto semi-direto externo, 97

propriedade do colarinho, 71

propriedade do ponto fixo, 110

Retrato de uma Vizinhança Euclideana, 71

seção, 103

seção local, 103 seqüência espectral do tipo homológica, 49

sequência de Mayer-Vietoris, 135

sequência espectral convergente, 50

Sequência espectral de Leray-Serre para

Homologia, 51

sequência espectral do primeiro

quadrante, 50

sequência espectral limitada, 50

sequencia exata curta escinde, 98

simplexo padrão, 48

subcategoria, 31

subcategoria completa, 31

superfície topológica, 77

suspensão não reduzida, 45

Teorema

de Seifert-Van Kampen, 134

Teorema da Monodromia, 142

Teorema dos Coeficientes Universais para Homologia, 52

topologia admissível, 33

topologia compacta-aberta, 32

transformação natural, 30

Variedade aberta, 70

variedade de Whitehead, 119

Variedade fechada, 70

variedade topológica, 44

variedade topológica com bordo, 68

variedade topológica com ou sem bordo, 70

variedade topológica sem bordo, 70

vizinhança de disco, 80 